\title{
LUDWIG-MAXIMILIANS-UNIVERSITÄT
}

\section{Quantum Field Theories}

\author{
Coupled to
}

\section{Supergravity}

AdS/CFT and Local Couplings

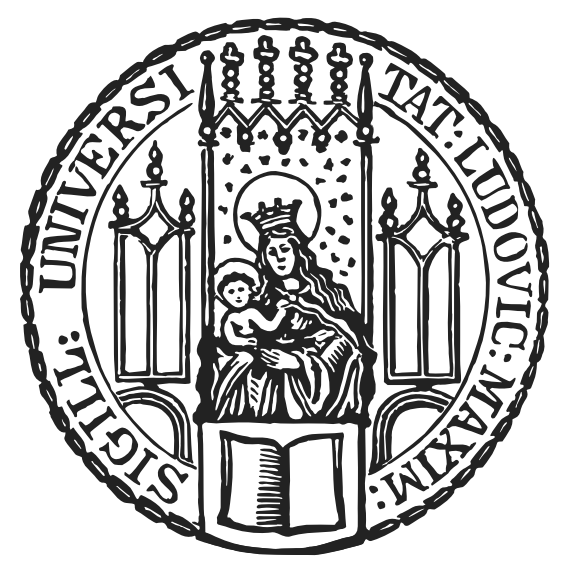

Johannes Große

Munich 2006 
(C) 2006 Johannes Große

Referee I: Priv.-Doz. Dr. Johanna Erdmenger

Referee II: Prof. Dr. Dieter Lüst

Rigorosum: November 8, 2006 


\title{
LUDWIG-MAXIMILIANS-UNIVERSITÄT
}

\section{Quantum Field Theories}

\author{
Coupled to
}

Supergravity

\author{
AdS/CFT and Local Couplings
}

\author{
A Dissertation \\ Presented to the Department for Physics of \\ Ludwig-Maximilians-Universität München \\ in Candidacy for the Degree of \\ doctor rerum naturalium by \\ Johannes Große \\ from Berlin
}

Munich, August 3, 2006 



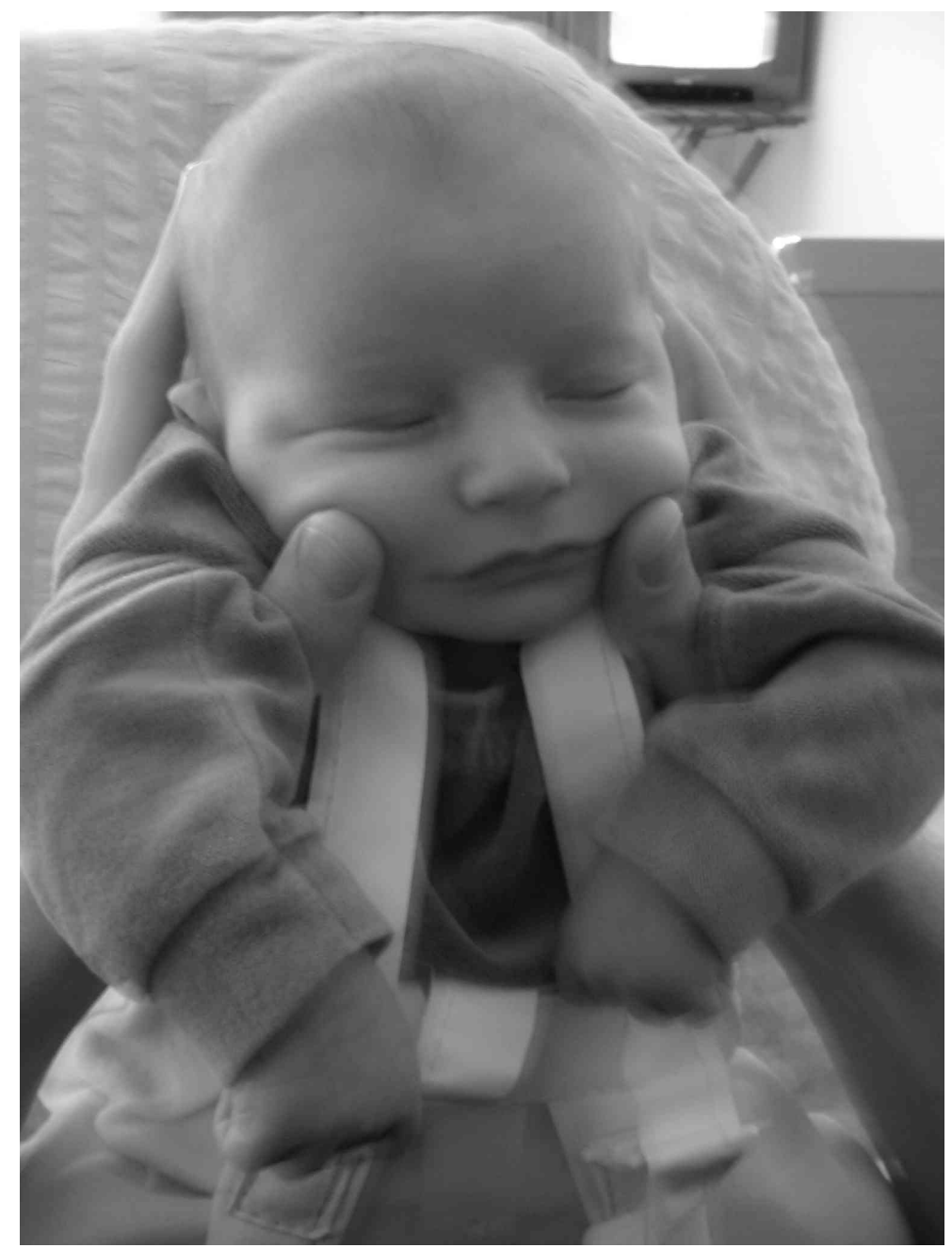

Dedicated to a true explorer 



\section{Zusammenfassung}

Diese Dissertation ist der Untersuchung des Zusammenspiels von supersymmetrischen Yang-Mills-Theorien (SYM) und Supergravitationstheorien (SUGRA) gewidmet. Das Thema wird von zwei Seiten beleuchtet:

Zunächst vom Standpunkt der AdS/CFT Korrespondenz, die die Kopplung zwischen vierdimensionaler superkonformer $\mathcal{N}=4$ SYM-Theorie und zehndimensionaler Typ IIB SUGRA holographisch realisiert. Um zu Theorien zu gelangen, die größere Ähnlichkeit mit Quantenchromodynamik (QCD) aufweisen, werden fundamentale Felder mit Hilfe von D7-Branen in die Korrespondenz eingeführt und nicht-triviale Hintergrundkonfigurationen betrachtet. Insbesondere werden Supergravitationslösungen verwendet, die nur noch asymptotisch die anti-de Sitter-Geometrie annähern, was Supersymmetrie bricht und die Beschreibung von spontaner chiraler Symmetriebrechung ermöglicht. Das Mesonspektrum wird berechnet und die Existenz einer zugehörigen Goldstone-Mode nachgewiesen sowie das nicht Auftreten der Entartung bei Mesonen hoher radialer Anregung. Darüberhinaus werden Instantonkonfigurationen auf den D7-Branen untersucht, die zu einer Beschreibung des Higgs branch der dualen Feldtheorie führen. Im Anschluss wird eine holographische Beschreibung von heavylight Mesonen entwickelt, die sich aus Quarks mit großem Massenunterschied zusammensetzen, was die Behandlung von B-Mesonen ermöglicht.

Als zweite Zugang zum Thema wird die Technik der sogenannten ortsabhängigen (auch: „lokalen“) Kopplungen gewählt, bei der die Kopplungs- 
konstanten zu externen Quellen erweitert werden, was die Untersuchung der konformen Anomalie von Quantenfeldtheorien, die an einen klassischen Gravitationshintergrund gekoppelt werden, ermöglicht. Diese Technik wird auf die Superfeldbeschreibung minimaler $\mathcal{N}=1$ Supergravitation ausgedehnt, eine Basis für die Anomalie angegeben und die Konsistenzbedingungen, die im Rahmen von Kohomologiebetrachtungen auftreten, berechnet. Mögliche Implikationen für eine Erweiterung von Zamolodchikovs $c$-Theorem auf vierdimensionale supersymmetrische Quantenfeldtheorien werden diskutiert. 
Who is General Failure and what did he do to my

thesis?

author unknown (due to technical problems)

\section{Abstract}

This dissertation is devoted to the investigation of the interplay of supersymmetric Yang-Mills theories (SYM) and supergravity (SUGRA). The topic is studied from two points of view:

Firstly from the point of view of AdS/CFT correspondence, which realises the coupling of four dimensional superconformal $\mathcal{N}=4$ SYM theory and ten dimensional type IIB SUGRA in a holographic way. In order to arrive at theories that resemble quantum chromodynamics (QCD) more closely, fundamental fields are introduced using probe D7-branes and nontrivial background configuration are considered. In particular supergravity solutions that are only asymptotically anti-de Sitter and break supersymmetry are used. This allows the description of spontaneous chiral symmetry breaking. The meson spectrum is calculated and the existence of an associated Goldstone mode is demonstrated. Moreover it is shown that highly radially excited mesons are not degenerate. Additionally instanton configurations on the D7-branes are investigated, which lead to a holographic description of the dual field theory's Higgs branch. Finally a holographic description of heavy-light mesons is developed, which are mesons consisting of quarks with a large mass difference, such that a treatment of B mesons can be achieved.

The second approach to the topic of this thesis is the technique of socalled space-time dependent couplings (also known as "local couplings"), where coupling constants are promoted to external sources. This allows to 
explore the conformal anomaly of quantum field theories coupled to a classical gravity background. The technique is extended to the superfield description of $\mathcal{N}=1$ supergravity, a complete basis for the anomaly is given and the consistency conditions that arise from a cohomological treatment are calculated. Possible implications for an extension of Zamolodchikov's $c$-theorem to four dimensional supersymmetric quantum field theories are discussed. 


\section{Contents}

Zusammenfassung vii

Abstract ix

Preface xix

Introduction $\quad$ xxi

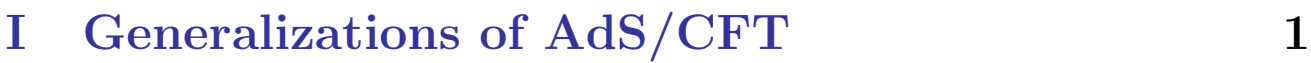

1 Overview 3

$1.1 \mathrm{QCD} \ldots \ldots \ldots \ldots \ldots \ldots \ldots$

$1.2 \mathcal{N}=4$ Super-Yang-Mills Theory . . . . . . . . . 5

1.3 Type IIB Supergravity . . . . . . . . . . . . . . 6

1.3.1 p-brane Solutions . . . . . . . . . . . . 8

1.4 D-branes . . . . . . . . . . . . . . . . . . . . . . . . 9

1.4.1 Abelian .................. . . 10

1.4 .2 Non-Abelian . . . . . . . . . . . . . . . 11

1.4 .3 Quadratic Action ............. . . 13

1.5 AdS/CFT Correspondence . . . . . . . . . . . . . 15

2 Spicing with Flavour $\quad 19$

2.1 Motivation .................. 20 
2.2 Probe Brane . . . . . . . . . . . . . . . . . . . . . . 21

2.3 Analytic Spectrum . . . . . . . . . . . . . . . 23

2.3.1 Fluctuations of the Scalars . . . . . . . . . . 23

2.3.2 Fluctuations of the Gauge Fields . . . . . . . 26

2.4 Operator Map . . . . . . . . . . . . . . . . . . . . . . 28

3 First Deformation: Geometry 31

3.1 Chiral Symmetry Breaking . . . . . . . . . . . . . . 31

3.2 DBI to Quadratic Order . . . . . . . . . . . . 35

3.3 Quadratic Fluctuations . . . . . . . . . . . . 36

3.4 Equations of Motion . . . . . . . . . . . . . . . . 38

3.4 .1 Vacuum . . . . . . . . . . . . . . 38

3.4.2 Pseudoscalar Mesons . . . . . . . . . . . . . 38

3.4.3 Scalar Mesons . . . . . . . . . . . . . . . . 38

3.4 .4 Vector Mesons . . . . . . . . . . . . . . . 39

3.5 Backgrounds . . . . . . . . . . . . . . . . . . . 39

3.5.1 $\mathrm{AdS}_{5} \times \mathrm{S}^{5} \ldots \ldots \ldots \ldots \ldots \ldots$

3.5.2 Gubser's Geometry . . . . . . . . . . . . 40

3.6 Chiral Symmetry Breaking in Gubser's Background . . . . 42

3.7 Mesons . . . . . . . . . . . . . . . . . . . 46

3.8 Highly Excited Mesons . . . . . . . . . . . . . . . . . . . . 48

4 Second Deformation: Gauge Fields 55

4.1 Introduction . . . . . . . . . . . . . . . . . . 55

4.2 Conventions . . . . . . . . . . . . . . . . 56

4.3 Dual Field Theory . . . . . . . . . . . . . . . 57

4.3 .1 Higgs Branch . . . . . . . . . . . . . 58

4.4 Supergravity . . . . . . . . . . . . . . . 60

4.4 .1 Instantons . . . . . . . . . . . . . . . . 60

4.4.2 D7-brane Action . . . . . . . . . . . . 62

4.5 Meson Spectrum . . . . . . . . . . . . . . . . . 64

4.5.1 Vector Fluctuations . . . . . . . . . . . . 65

4.5.2 Scalar Fluctuations . . . . . . . . . . . 71

5 Heavy-Light Mesons $\quad 73$

5.1 Heavy-Light Mesons in $\mathrm{AdS}_{5} \times \mathrm{S}^{5} \ldots \ldots . . . . . . .74$ 
5.2 Dilaton Flow Geometries . . . . . . . . . . . . . . 80

5.2.1 Gubser's Dilaton Deformed Geometry . . . . . . . . 81

5.2.2 Constable-Myers' Background . . . . . . . . . . . . 85

5.3 Bottom Phenomenology . . . . . . . . . . . . . 88

\section{Space-time Dependent Couplings $\quad 93$}

6 Supergravity Overview $\quad 95$

6.1 Conventions . . . . . . . . . . . . . . . . . 96

6.2 Superspace Supergravity . . . . . . . . . . . . . . . . 102

6.3 Non-minimal Supergravity . . . . . . . . . . . . . . . . . . 104

6.3.1 Algebra and Bianchi identities . . . . . . . . . . 105

6.3.2 Partial Integration . . . . . . . . . . . . . . 106

6.3.3 Superdeterminant . . . . . . . . . . . . . . 107

6.3.4 Super-Weyl Transformations . . . . . . . . . . . . . 108

6.3.5 Prepotentials . . . . . . . . . . . . . . 109

6.4 Minimal Supergravity . . . . . . . . . . . . . . . . . 110

6.4.1 Algebra and Bianchi Identities . . . . . . . . . . . . 110

6.4.2 Chiral Projector and d'Alembertian . . . . . . . . 111

6.4.3 Super-Weyl Transformations . . . . . . . . . . . . . 112

6.4.4 Chiral Representation and Integration Rule . . . . 114

6.5 Component Expansion . . . . . . . . . . . . . . . . 115

6.5.1 Superfields and First Order Operators . . . . . . . 115

6.5.2 Supergravity Fields . . . . . . . . . . . . . . 117

6.5.3 Full Superspace Integrals . . . . . . . . . . . . . . . 119

7 Space-Time Dependent Couplings $\quad 121$

7.1 Weyl Transformations . . . . . . . . . . . . . . . . 122

7.1.1 Conformal Killing Equation . . . . . . . . . . . . 122

7.1.2 Conformal Algebra in $d>2 \ldots \ldots$. . . . . . 123

7.1.3 Weyl Transformations of the Riemann Tensor . . . 124

7.1.4 Weyl Covariant Differential Operators . . . . . . 125

7.2 Zamolodchikov's c-Theorem in Two Dimensions . . . . . . 127

7.3 Conformal Anomaly in Four Dimensions . . . . . . . . . . 129

7.4 Local RG Equation and the $c$-Theorem . . . . . . . . . . . 130 
7.4.1 a-Theorem . . . . . . . . . . . . 133

8 Supersymmetric Trace Anomaly 135

8.1 SUSY Local RG Equation . . . . . . . . . . . . . . . . . . 135

8.2 Basis for the Trace Anomaly . . . . . . . . . . . . . . 137

8.3 Wess-Zumino Consistency Conditions . . . . . . . . . . . 141

8.4 Local Counterterms . . . . . . . . . . . . . . . . 143

8.5 S-duality . . . . . . . . . . . . . . . . . . 144

8.6 Towards a Proof . . . . . . . . . . . . . . . . 146

8.7 Superfield Riegert Operator . . . . . . . . . . . . . . 147

8.8 Discussion . . . . . . . . . . . . . . . . . . . . . . . . . . 150

$\begin{array}{ll}\text { Conclusions } & 155\end{array}$

$\begin{array}{ll}\text { Acknowledgements } & 163\end{array}$

A Determinant Expansion 165

$\begin{array}{ll}\text { B Weyl Variation of the Basis } & 171\end{array}$

C Wess-Zumino Consistency Condition 175

$\begin{array}{ll}\text { D Coefficient Consistency Equations } & 181\end{array}$

$\begin{array}{ll}\text { E Minimal Algebra on Chiral Fields } & 187\end{array}$

$\begin{array}{ll}\text { Curriculum Vitæ } & 203\end{array}$ 


\section{List of Figures}

1.1 Double Line Representation . . . . . . . . . . . . . . . 16

2.1 Terminating D7-brane ............... 24

3.1 Regularity Conditions . . . . . . . . . . . . . . 33

3.2 Breaking of the Axial Symmetry . . . . . . . . . . . 34

3.3 D7-brane Embeddings . . . . . . . . . . . . 43

3.4 Chiral Condensate in Dilaton Deformed Background . . . . 44

3.5 Singularity Shielding . . . . . . . . . . . . . . 46

3.6 D7-brane Action and Physical Solution . . . . . . . . . 47

3.7 Lightest Scalar, Pseudoscalar and Vector Meson . . . . . . 49

3.8 Highly Excited Mesons (Overview) . . . . . . . . . . 50

3.9 Close-up of a Highly Radially Excited Meson . . . . . . . . 53

4.1 Meson Masses on the Higgs Branch . . . . . . . . . . . 67

4.2 Meson Masses on the Higgs Branch 2 . . . . . . . . . . 70

5.1 D3/D7 Geometry . . . . . . . . . . . . . 75

5.2 Heavy-Light Brane Configuration . . . . . . . . . . 76

5.3 Heavy-Light Meson Spectrum in AdS . . . . . . . . . . . 78

$5.4 \mathrm{H} / \mathrm{L}$ Meson Spectrum in AdS (small 't Hooft coupling) . . 79

5.5 Vacuum Embeddings . . . . . . . . . . . . . . . . . 82

5.6 Binding Energy in Gubser's Background . . . . . . . . . . 84

5.7 Binding Energy in Constable-Myers' Background . . . . . 87 
5.8 Mesons in Gubser's Background (reviewed) . . . . . . . . . 89

5.9 B/B* Mass Ratio . . . . . . . . . . . . . . . . . . 90

7.1 Limit Cycle in the Space of Couplings . . . . . . . . . . . . 128 


\section{List of Tables}

1.1 Quark Masses (PDG) . . . . . . . . . . . . . 4

1.2 IIB SUGRA Particle Content . . . . . . . . . . . . . 7

1.3 Transversal vs. Longitudinal Coordinates . . . . . . . . . . 10

2.1 D3/D7-brane Embedding . . . . . . . . . . . . . . 21

2.2 Mesonic Spectrum in $\mathrm{AdS}_{5} \times \mathrm{S}^{5} \ldots \ldots . . \ldots 29$

4.1 Index Conventions . . . . . . . . . . . . . . . 57

6.1 Definition of Conjugations . . . . . . . . . . . . . 100

6.2 Superfield Supergravity Index Conventions . . . . . . . . . 102

7.1 Conformal Transformations . . . . . . . . . . . . . 123

8.1 Dimensional Analysis for Supergravity Fields . . . . . . . . 138 

Success is the ability to go from one failure to another with no loss of enthusiasm.

Sir Winston Churchill

\section{Preface}

The work described in this thesis was carried out in collaboration with Dr. Johanna Erdmenger, Dr. Zachary Guralnik and Dr. Nick Evans. This thesis covers the work presented in the following publications [1-4].

- J. Erdmenger, N. Evans, J.G., "Heavy-Light Mesons from the AdS/ CFT Correspondence," JHEP 0701, 098 (2007) [hep-th/0605241].

- R. Apreda, J. Erdmenger, N. Evans, J.G., Z. Guralnik, "Instantons on D7-brane probes and AdS/CFT with flavour," Fortsch. Phys. 54, 266 (2006) [hep-th/0601130].

- J. Erdmenger, J.G., Z. Guralnik, "Spectral flow on the Higgs branch and AdS/CFT duality," JHEP 0506, 052 (2005) [hep-th/0502224].

- J.G., "MathPSfrag: Creating Publication-Quality Labels in Mathematica Plots," submitted to Computer Physics Communications, cs.GR/0510087.

Moreover the results of Chapter 3, which constitute independent work, have been presented in a talk at the "XVI. Workshop Beyond The Standard Model," Bad Honnef, March 8-11, 2004.

No claims to originality are made for the content of Chapters 1, 2, 6 and 7 , which were compiled using a variety of sources. 

Art is born of the observation and investigation of nature.

Cicero

\section{Introduction}

An important goal of theoretical physics is the algorithmic compression of nature to a set of fundamental laws. This means that a minimal description is sought that encodes a maximum of information about our universe. At the current state of knowledge, this description is in terms of the standard model of elementary particles and Einstein gravity, as well as initial conditions and parameters. Although many models used in other areas of physics are not derived from those fundamental theories, in principle such a derivation should nevertheless be possible.

The standard model is a quantum field theory that describes elec- standard model tromagnetism, the weak and the strong force, organised by the principle of gauge invariance. The latter arises from making the formulation manifestly Lorentz invariant which requires the introduction of extra nonphysical degrees of freedom. Consequently there are many representations of the same physical state, which are related by so-called gauge transformations. Gauge transformations can be identified with Lie groups having space-time dependent parameters and form the internal symmetry group of the standard model, the group $\mathrm{U}(1) \times \mathrm{SU}(2) \times \mathrm{SU}(3)$, corresponding to quantum electrodynamics (QED) describing photons, the weak interaction, whose gauge fields are the $\mathrm{W}$ and $\mathrm{Z}$ bosons responsible for the $\beta$ decay, and quantum chromodynamics (QCD), the theory of the strong force, which describes the constituents of hadrons like the proton and the neutron.

QED and

We shall first have a closer look at QED, which is a remarkably success- renormalisation 
ful theory, confirmed to an incredible accuracy of up to $10^{-11}$ over the past decades. Since a rigorous treatment of interacting quantum field theories is difficult, an important reason for this success is the possibility to treat QED perturbatively. In perturbation theory a theory is effectively split into a solvable part; e.g. a free theory, and the remainder that renders the theory unsolvable; e.g. the interaction terms. Assuming that the solutions of the free theory are only slightly modified by the presence of the additional interaction terms allows an expansion in the coupling constant. However this expansion is not a true series expansion since the coupling constants themselves need to be modified during the expansion by a procedure called renormalisation to absorb infinite contributions arising from the interplay of the quantisation procedure and perturbation theory. Theories allowing to absorb these infinities in a finite number of parameters are called renormalisable and can be treated perturbatively in a well defined manner.

There are basically two points where this strategy can fail and interestingly both have a connection to string theory as will be seen later.

non-renormalisable theories

The first problem arises when trying to tackle non-renormalisable theories like gravity. Each order of perturbation theory then produces a growing number of coupling constants that destroy the predictive power of the theory. This can either be interpreted as there being something wrong with the quantisation procedure assuming that gravity has some miraculous ultraviolet (UV) behaviour that is merely poorly understood or that Einstein gravity is just an effective field theory that breaks down when leaving its regime of validity (at the order of the Planck mass $m_{P} \approx 10^{19} \mathrm{GeV}$ ) and a more fundamental theory is required.

In the spirit of the introductory remarks at the beginning, such a "more fundamental" theory, from which also the standard model of elementary particles should be derived, is a natural goal, which unfortunately seems to be currently out of reach. However there exists at least a candidate theory that consistently quantises gravity and at the same time incorporates gauge theories similar to the standard model, namely superstring theory. Entertainingly this extremely remarkable feature was not what led to its discovery and it is also not the feature central to this thesis, which shall be explicated in the followings. 
The second problem of perturbation theory arises from the phenome- strong coupling non of running gauge couplings, a result - though not a consequence - of renormalisation. It is the statement that the strength of the interaction and thus the validity of perturbation theory depends on the energy scale. While the electroweak force has small coupling constants at low energies, which become large when going to higher energies, the opposite is true for QCD, which is asymptotically free. For small energies QCD exhibits a phase transition, the confinement, that effectively screens the theory's fundamental particles, the quarks and gluons, from the dynamics by creating bound states of vanishing colour charge: hadrons. In that sense QCD is an accelerator theory that can only be observed at high energies, although there is very strong evidence from lattice calculations that QCD is also the correct theory for low energies where ordinary perturbation theory is not applicable and the dominating degrees of freedom are better recast in an effective field theory. However a better understanding of the low-energy dynamics of QCD and confinement is still sought after.

Before the break-through of QCD there was another candidate theory for the strong interaction, which could reproduce certain relations in the spectra of low energy hadron physics: string theory.

String theory describes particles as oscillation modes of strings that string theory propagate through space-time, joining and splitting along their way, thus sweeping out a two-dimensional surface, the world-sheet. The action of a string is that of an idealised soap film; i.e. proportional to the area of the world sheet. Another interesting feature of the low energy dynamics of hadrons is the formation of flux tubes between quarks, which are also string like and even though nowadays perfectly understandable from a pure QCD point of view seemed to hint at a connection between string theory and hadron physics. As will be seen later this connection does indeed exist in the form of the 't Hooft large $N_{c}$ expansion [5], which was born in 't Hooft an attempt to find a small parameter for perturbative calculations in the expansion strong coupling regime. The basic idea is to look at $\mathrm{SU}\left(N_{c}\right)$ Yang-Mills theories, where $N_{c}$ is the number of colours, ${ }^{*}$ and perform an expansion in $\frac{1}{N_{c}}$. This implies at leading order the 't Hooft limit $N_{c} \rightarrow \infty$, where additionally $\lambda:=g_{Y M}^{2} N_{c}$ is kept fixed, with $g_{Y M}$ the Yang-Mills coupling

${ }^{*}$ For $N_{c}=3$ this describes the pure glue part of QCD. 
constant. This particular choice is motivated by keeping the strong coupling scale $\Lambda_{Q C D}$ constant in a perturbative calculation of the $\beta$ function. In a double line notation, the diagrams associated to each order in $\frac{1}{N_{c}}$ can be seen to give rise to a topological expansion, which can be interpreted as a triangulation of two dimensional manifolds, the string world sheets in a genus expansion. While this triangulation is not understood in detailsee [6] for recent approaches to this important point - there is nevertheless a map between a particular gauge theory and string theory in a certain background.

AdS/CFT This map, tested by a large number of highly non-trivial checks, is Maldacena's conjecture [7] of AdS/CFT correspondence. In its boldest form, it is the statement that $\mathcal{N}=4$ super-Yang-Mills (SYM) theory, which is a conformal field theory (CFT) is dual to (quantised) type IIB string theory on $\mathrm{AdS}_{5} \times \mathrm{S}^{5}$. By "dual" the existence of a map is meant that identifies correlation functions of both theories, thus rendering them actually two different pictures of the same theory. The details will be reviewed in Chapter 1. For now it is sufficient to remark that string theory in that particular background is still ill-understood, but that there are limits in which things are better under control. In the string loop expansion, each hole in the world sheet comes with a factor of $g_{s}$, while in a similar gauge theory Feynman diagram each hole corresponds to a closed loop and is therefore accompanied by a factor of $g_{Y M}^{2}$. This naïve analysis allows to identify $g_{Y M}^{2}=g_{s}$, which therefore go to zero simultaneously in the 't Hooft limit, demonstrating that the $\frac{1}{N_{c}}$ expansion corresponds to a genus expansion of the string world sheet.

From the construction of the $\mathrm{AdS}_{5} \times \mathrm{S}^{5}$ background in type IIB supergravity (SUGRA) theory, which is the small curvature, low energy limit of type IIB superstring theory, it is possible to derive the relation $\left(\frac{L}{\ell_{s}}\right)^{4} \sim \lambda$, where $L$ is the respective curvature radius of the anti-de Sitter space $\left(\mathrm{AdS}_{5}\right)$ and the five-sphere $\left(\mathrm{S}^{5}\right)$, and $\ell_{s}=\sqrt{\alpha^{\prime}}$ is the string length.

Therefore, the limit of small curvature $L \gg \ell_{s}$, where type IIB supergravity on $\mathrm{AdS}_{5} \times \mathrm{S}^{5}$ is a good approximation of the corresponding string theory, is dual to taking $\lambda$ large in the field theory. Because $\lambda$ takes over the rôle of the coupling constant in the large $N_{c}$ limit, with $\lambda \ll 1$ the perturbative regime, the duality relates said supergravity theory to 
strongly coupled $\mathcal{N}=4 \mathrm{SYM}$ theory in the large $N_{c}$ limit. Since the discovery of the actual mapping prescription between correlators on both sides of the correspondence $[8,9]$, a plethora of non-trivial checks have been performed $[10,11]$, that not only extended the correspondence to less symmetric regimes but also provided overwhelming evidence that the conjecture actually holds true.

This thesis is devoted to studying the coupling between supergravity (SUGRA) theories and quantum field theories. Although the idea was QFT coupled to revived by the discovery of AdS/CFT duality, where this coupling is realised holographically, that is between a four and a five dimensional theory, it has also been considered earlier in the context of space-time dependent coupling constants $[12-14]$.

In the first part of this thesis several aspects of AdS/CFT correspondence will be discussed, while the second part uses the idea of space-time dependent couplings to analyse the conformal anomaly in super-YangMills theories coupled to minimal supergravity.

Since at a first glance these two subjects seem rather unrelated, I would like to linger on a bit on the question of what the two topics have in common before continuing the introduction to those two parts.

The idea of space-time dependent couplings is to promote coupling space-time constants to (external) fields. Generically the coupling takes the form $\int d^{4} x \mathcal{J O}$, where $\mathcal{J}$ acts as a source for the operator $\mathcal{O}$. A particularly dependent important example for such a source/operator pair is the metric and the energy-momentum tensor, which couple according to

$$
S \mapsto S+\int d^{4} x g^{m n} T_{m n}
$$

such that allowing coordinate dependence $g^{m n}=g^{m n}(x)$ amounts to coupling the quantum field theory to a (classical) gravity background - or a supergravity background for supersymmetric quantum field theories. Invariance of the action under diffeomorphisms $\delta g^{m n}=\mathcal{L}_{v} g^{m n}$ implies $\nabla^{m} T_{m n}=0$, while from Weyl invariance $\left(\delta g^{m n}=2 \sigma g^{m n}\right)$ one may conclude $T_{m}{ }^{m}=0$. When quantum effects destroy the Weyl symmetry of a classical theory, the trace of the energy-momentum tensor does not vanish anymore. It is said to have an anomaly: the Weyl or trace anomaly, which 
is a standard example of a quantum anomaly. More will be said about it below.

AdS/CFT mapping of correlation functions$$
\text { terms of SUGRA fields is given by }
$$

$$
\left\langle\exp \int d^{4} x \phi^{(0)} \mathcal{O}\right\rangle_{\mathrm{CFT}}=\left.\exp \left\{-S_{\mathrm{SUGRA}}[\phi]\right\}\right|_{\phi(\partial \mathrm{AdS})=\phi^{(0)}}
$$

where the right hand side is the generating functional of the classical supergravity theory, which is evaluated with its fields $\phi$ determined by their equations of motion and their boundary values $\phi^{(0)}$ that appear as sources for field theory operators in the CFT.

AdS/QCD? Much of the excitement about the AdS/CFT duality came from the prospect of gaining insight into the strong coupling regime of Yang-Mills (YM) theories and QCD. Both $\mathcal{N}=4$ SYM and type IIB SUGRA are (almost) entirely determined by their large symmetry group, namely $\mathrm{SU}(2,2 \mid 4)$. For the mapping of operators on both sides, this is a beautiful feature, but non-supersymmetric YM has a much smaller field content and the problem arises how to get rid of the extra fields. Furthermore to describe QCD quarks are needed but $\mathcal{N}=4$ SYM contains only one hypermultiplet whose gauge field forces its adjoint representation on all other fields.

The conformal group $\mathrm{SO}(2,4)$ of the CFT corresponds to the isometry group of $\mathrm{AdS}_{5}$. Similarly the $\mathrm{SU}(4)_{R}$ group is matched by the $\mathrm{SO}(6)$ isometry group of the $\mathrm{S}^{5}$. Therefore a less supersymmetric CFT will be dual to a SUGRA on $\mathrm{AdS}_{5} \times \mathrm{M}^{5}$, where $\mathrm{M}^{5}$ is a suitable less symmetric manifold. Unfortunately the operator map relies heavily upon the field theory operators being uniquely determined by their transformational behaviour under the global symmetry groups, such that reducing the symmetry implies making the correspondence less precise. This is especially true when also giving up the conformal symmetry in order to obtain discrete mass spectra.

deformed AdS/CFT

Therefore the strategy employed in this thesis will be to describe theories that are very symmetric in the UV but are relevantly deformed and flow to a less symmetric, phenomenologically more interesting non-conformal 
infrared (IR) theory. This allows to still use the established AdS/CFT correspondence while at the same time capturing interesting IR physics.

Such a renormalisation group (RG) flow is represented by a supergravity solution that approaches an AdS geometry only towards the boundary, it is asymptotically AdS. The interior of the deformed space corresponds to the field theoretic IR. The interpretation of the radial direction of the (deformed) AdS space as the energy scale can be easily seen from considering dilations of the boundary theory. Since the boundary theory is conformal such a dilation should leave the action invariant. To achieve the same in the SUGRA theory, the radial direction has to transform as an energy to cancel in the metric the transformation of the coordinates parallel to the boundary. The interpretation of the radial direction as the renormalisation scale was introduced in $[15,16]$ and has been used for a number of checks of the AdS/CFT duality, for example calculation of the ratio of the conformal anomaly at the fixed points of holographic RG flows $[10,17]$, which coincides with field theory predictions.

An important step towards a holographic description of QCD is the quarks introduction of fundamental fields into the correspondence. The first realisation of such a theory was a string theory in an $\mathrm{AdS}_{5} \times \mathrm{S}^{5} / \mathbb{Z}_{2}$ background where a number of D7 branes wrapped the $\mathbb{Z}_{2}$ orientifold plane with geometry $\mathrm{AdS}_{5} \times \mathrm{S}^{3}[18,19]$, which is dual to an $\mathcal{N}=2 \operatorname{Sp}\left(N_{c}\right)$ gauge theory. As was realised by [20], a similar scenario of probe D7-branes wrapping a contractible $\mathrm{S}^{3}$ in $\mathrm{AdS}_{5} \times \mathrm{S}^{5}$ leads to a consistent description of an $\mathcal{N}=2 \mathrm{SU}\left(N_{c}\right)$ theory, since a contractible $\mathrm{S}^{3}$ does not give rise to a tadpole requiring cancellation, nor to an unstable tachyonic mode due to the Breitenlohner-Freedman bound [21]. (Further extensions of AdS/CFT using D7 branes to include quarks have been presented in [1, 2228].*) The full string picture is that of a D3-brane stack, whose near horizon geometry gives rise to an $\mathrm{AdS}_{5} \times \mathrm{S}^{5}$ space, probed by parallel D7-branes wrapping and completely filling an $\mathrm{AdS}_{5} \times \mathrm{S}^{3}$ geometry. The probe D7-branes strings connecting the two stacks give rise to an $\mathcal{N}=2$ hypermultiplet in the fundamental representation. The resulting field theory is conformal as long as the two brane stacks coincide. In this case the setup preserves an $\mathrm{SO}(4) \times \mathrm{SO}(2)$ subgroup of the original $\mathrm{SO}(6)$ isometry, which is dual

*Related models involving other brane setups may be found in [29-37]. 
to an $\mathrm{SU}(2)_{L} \times \mathrm{SU}(2)_{R} \times \mathrm{U}(1)_{R}$ subgroup of the $\mathrm{SU}(4)_{R}$.

Separating the two stacks introduces a quark mass and breaks conformal symmetry as well as the $\mathrm{SO}(2) \simeq \mathrm{U}(1)_{R}$ symmetry. Consequently the induced geometry on the D7-branes becomes only asymptotically $\mathrm{AdS}_{5}$. At the same time, the $S^{3}$ starts to slip of the internal $S^{5}$ when approaching the interior of the $\mathrm{AdS}_{5}$ and shrinks to zero size. At that point the quarks decouple from the IR dynamics and the D7-brane seems to end from a five dimensional point of view. By solving the Dirac-Born-Infeld (DBI) equations of motion for the fluctuations of the D7 branes about their embedding the meson spectrum can be determined [24]. The setup is reviewed in more detail in Chapter 2.

deformed In Chapter 3, I discuss how to combine the ideas laid out above, that is background to consider probe D7-branes in background geometries that only approach geometry $\mathrm{AdS}_{5} \times \mathrm{S}^{5}$ asymptotically. The specific geometry under consideration is that of a dilaton flow by Gubser [38], which preserves an $\mathrm{SO}(1,3) \times \mathrm{SO}(6)$ isometry while breaking conformal invariance and supersymmetry, thereby allowing chiral symmetry breaking by the formation of a bilinear quark condensate.

In the framework of AdS/CFT correspondence all supergravity fields encode two field theoretic quantities, a source and a vacuum expectation value (VEV). The embedding of a probe D7-brane is determined by a scalar field arising from the pullback of the ambient metric to the world volume of the brane. Solving the equation of motion for this scalar field $\Phi$ yields the following UV behaviour,

$$
\Phi \sim m_{q}+\frac{\langle\bar{\psi} \psi\rangle}{\rho^{2}}
$$

where $\rho$ is the radial coordinate of the AdS space, whose boundary is approached for $\rho \rightarrow \infty$.

Extending the solution to the interior of the space, it turns out that generic combinations of the quark mass $m_{q}$ and the chiral condensate $\langle\bar{\psi} \psi\rangle$ do not produce solutions that have a reasonable interpretation as a field theoretic flow; i.e. are expressible as a function of the energy scale $\rho$. I demonstrate that this requirement is sufficient to completely fix the condensate as a function of the quark mass. In the limit of vanishing 
quark mass there is a non-vanishing bilinear quark VEV indicating that the background is indeed a holographic description of spontaneous chiral symmetry breaking.

I then determine the mass of the lowest scalar, pseudoscalar and vector meson by calculating the fluctuations about the embedding solutions. Since the equations of motion for the D7 embedding in the deformed background could only be solved numerically, the same holds true for the fluctuations about these vacuum solutions. Still the spectrum is well understood because it approaches the analytic solutions of the supersymmetric case in the limit of large quark mass. This is to be expected since for larger quark mass, the corresponding mesons decouple from the dynamics at high energies where supersymmetry is restored. I show that in the limit of vanishing quark mass, where chiral symmetry is broken spontaneously, the pseudoscalar meson becomes massless and is therefore a Goldstone boson for the axial symmetry. For small quark mass $m_{q}$, the mass of the Goldstone mode essentially behaves like $\sqrt{m_{q}}$ in accordance with predictions from effective field theory.

Moreover I discuss the spectrum of highly radially excited mesons (as opposed to excitations on the $\mathrm{S}^{3}$, which are not in mutually same representations of $\left.\mathrm{SU}(2)_{L} \times \mathrm{SU}(2)_{R}\right)$. It is explained why in this holographic setup (as in many others [39]) the field theoretic expectation [40, 41] of chiral symmetry restoration cannot be met. The reason is the infrared being probed more densely in the limit of large radial excitations, which also has an interesting effect on the heavy-light spectra discussed below.

In Chapter 4 instead of considering a non-trivial geometry, I discuss the effects of a non-trivial gauge field configuration on the brane. The non-trivial gauge spectrum of $N_{f} \ll N_{c}$ coincident D7-branes is described by a non-Abelian DBI action plus Wess-Zumino term $C_{4} \wedge F \wedge F$. Both scalar and vector fields on the brane are now matrix valued. Assuming that the branes are coincident one may diagonalise and obtain effectively $N_{f}$ copies of the spectrum of a single brane - unless there is a contribution from the Wess-Zumino term. This requires to choose a background configuration with non-trivial second Chern class; i.e. an instanton solution, which I demonstrate to indeed minimise the D7-brane action.

The string connecting the D7 and D3-branes separated by a distance 
$\left(2 \pi \alpha^{\prime}\right) m_{q}$ introduces a massive $\mathcal{N}=2$ hypermultiplet in the fundamental representation, which contributes the term $\tilde{Q}_{i}\left(m_{q}+\Phi_{3}\right) Q^{i}$ to the superpotential. $\tilde{Q}_{i}$ and $Q^{i}$ form the fundamental hypermultiplet and $\Phi_{3}$ is the chiral field that is part of the adjoint $\mathcal{N}=2$ gauge multiplet. The scalar component of $\Phi_{3}$ is an $N_{c} \times N_{c}$ matrix. If some of its elements acquire a VEV such that $m_{q}+\Phi_{3}$ is zero, then the corresponding components of the fundamental field may also get a VEV and the theory is on the mixed Coulomb-Higgs branch. I show that this Higgs VEV corresponds to the instanton size of above background and calculate the spectrum of scalar and vector mesons as a function of the Higgs VEV. In the limit of vanishing Higgs VEV I reproduce the analytic spectrum of the $\mathrm{SU}\left(N_{c}\right)$ gauge theory. Not surprisingly there is a sense in which the spectrum of an infinitely large Higgs VEV is equivalent since it belongs to an $\mathrm{SU}\left(N_{c}-1\right)$ gauge theory. I show that this equivalence holds only up to a non-trivial rearrangement of the spectrum by a singular gauge transformation.

In Chapter 5 mesons consisting of a light and a heavy quark are discussed. A naïve approach would be to use the non-Abelian DBI action, where the diagonal elements of the matrix valued scalar field now encode a mass and bilinear condensate for each of the corresponding $N_{f}$ quarks. Offdiagonal elements of the embedding solution would contain mass-mixing terms and mixed condensates, which one could set to zero for phenomenological reasons. Fluctuations about these embeddings would correspond to the ordinary same-quark meson for the diagonal elements and to heavylight mesons for the off-diagonal entries. However the latter are not small with respect to the corresponding light quark and expansion of the DBI action to quadratic order is not possible anymore. This step however is crucial to obtain an eigenvalue equation for the meson mass.

The approach chosen here is to find an effective description for heavylight mesons from the Polyakov action of the string stretched between two D7-branes with different separation from the D3 branes corresponding to two different quark masses. The separation is assumed to be large (that is only one quark is heavy, the light quark is taken massless), such that a semi-classical description of this long string is possible. I take the ansatz of a rigid string spanned in the direction of the separation of the two branes. The string is not allowed to oscillate or bend but only to move along the 
world volume of the D7s. Then integration over the string length can be carried out to obtain an effective point-particle-like action. Its equation of motion is a generalisation of the Klein-Gordon equation which can be quantised. I evaluate the resulting eigenvalue equation for the undeformed AdS background as well as dilaton deformed backgrounds by Gubser [38] and Constable-Myers [42].

The heavy-light meson spectrum for both deformed geometries approximates the AdS heavy-light spectrum for large quark mass. This behaviour is expected because a large quark mass corresponds to the string probing larger parts of the space-time that are approximately AdS. At the same time, it can be observed that highly excited mesons converge more slowly to their AdS values. Again this is in accordance with previous results of Section 3.8, where it has been demonstrated that highly excited mesons probe the IR region of the space time more densely, where the deviation from the AdS geometry is large.

These heavy-light spectra can be used to determine the mass of the B physics B meson by using the results of Chapter 3 as well as the experimental values of the Rho and Upsilon meson mass to fix the confinement scale and heavy quark mass. The prediction for the B mesons is $20 \%$ above the experimental value. Since the B mesons are far in the supersymmetric regime of this holographic model while at the same time the field theory is strongly coupled at that scale, this level of agreement is surprisingly good.

The AdS/CFT models I considered here describe chiral symmetry break- summary ing, highly excited mesons, the Higgs branch and heavy-light mesons, respectively. They have in common that they are not focused on building a perfect QCD dual, but instead are used to investigate particular features of YM theory with matter. The strategy of keeping a connection to standard AdS/CFT with flavours worked out and the results show either the qualitative behaviour expected from field theoretic and SUGRA considerations or could even be matched quantitatively to analytic results in certain limits.

As already mentioned this thesis consists of two parts. In the first part presented so far various aspects of AdS/CFT correspondence have been discussed and a number of models extending the AdS/CFT correspondence to theories 
with fundamental quarks have been developed and explored. The second part is devoted to an analysis of the conformal anomaly in super-Yang-Mills theories coupled to minimal supergravity in four space-time dimensions. This analysis is aimed at providing building blocks for a future generalisation of the two dimensional $c$-theorem, see below, to four-dimensional supersymmetric field theories.

trace anomaly

The conformal anomaly expresses the breaking of conformal invariance in a classically conformal field theory by quantum effects. It arises as the trace of the energy-momentum tensor, which - as mentioned abovevanishes in a conformally invariant theory, and is also called trace anomaly, hence.

c-theorem An investigation of the trace anomaly is interesting because of its potential relation to a four dimensional version of Zamolodchikov's $c$-theorem [43]. The $c$-theorem is a statement about the irreversibility of renormalisation group flows connecting two fixed points of a quantum field theory in two space-time dimensions. To be more precise the theorem states the existence of a monotonic function that at the fixed points, where the $\beta$ functions vanish, coincides with the trace anomaly coefficient $c$ defined by

$$
\left\langle T_{m}{ }^{m}\right\rangle=\frac{c}{24 \pi} \mathcal{R}
$$

where $\mathcal{R}$ is the scalar curvature. Moreover the coefficient $c$ turns up as the central charge of the Virasoro algebra and in the two point function of the energy-momentum tensor.

The $c$-theorem is also interesting from a philosophical point of view, because the $c$-function is interpreted to measure the number of degrees of freedom along the RG flow. Suppose that one believes that in the real world this number should be non-increasing when going to lower energies, a future "theory of everything" should certainly incorporate a function that measures these degrees of freedom and is monotonic hereby. While it is not clear that such an irreversibility theorem should be realised in terms of a $c$-theorem, the questions remains if there is a class of theories in four dimensions where an analogous statement to the two dimensional $4 D \quad c$-theorem can be made. Such a generalisation is not straight forward since 
conformal symmetry in four dimensions is far less powerful because the conformal algebra contains only a finite number of generators.

In four dimensions the trace anomaly reads

$$
\left\langle T_{m}{ }^{m}\right\rangle=c C^{2}-a \tilde{\mathcal{R}}^{2}+b \mathcal{R}^{2}+f \square \mathcal{R},
$$

with $C^{2}, \tilde{\mathcal{R}}^{2}$ and $\mathcal{R}^{2}$ respectively the square of the Weyl tensor, the Euler density and the square of the Ricci scalar $\mathcal{R}$. The first question that arises is which of these coefficients is to take over the rôle of the two dimensional $c$. While $f$ can be removed by adding a local counterterm to the quantum effective action, $c$ is known to be increasing in some theories and decreasing in others and $b$ is eliminated by Wess-Zumino consistency conditions. For the remaining coefficient, conventionally denoted " $a$ ", there is no known counterexample to $a_{\mathrm{UV}}>a_{\mathrm{IR}}$, though explicit checks can only be performed in certain classes of supersymmetric field theories [44, 45]. This might be an indication that supersymmetry is a necessary ingredient for such an $a$-theorem. The prospect of an $a$-theorem [46] has attracted some interest in the recent past under the name $a$-maximisation [47].

In this thesis a different approach inspired by an alternative proof of the $c$-theorem in two dimensions is chosen [48]. The author of [48] couples a quantum field theory that is conformal to a classical gravity background space-time dependent and investigates the anomaly arising from that coupling by promoting the coupling constants $\lambda$ to external fields $\lambda(x)$.

This trick yields well-defined operator insertions from functional derivations of the generating functional with respect to the couplings. A generalisation of the Callan-Symanzik equation to Weyl rescalings is found, which becomes anomalous when Weyl symmetry is broken upon quantisation. The structure of this equation is $\Delta_{\sigma} W=\mathcal{A}$, where $\Delta_{\sigma}$ contains a Weyl scaling part and a $\beta$ function part in analogy to the case of constant couplings and constant scale transformations.

The shape of the anomaly $\mathcal{A}$ is determined by dimensional analysis, anomaly ansatz yielding an ansatz that is a linear combination between a set of coefficient functions, which only depend on the couplings, and a set of basis terms, which depend on the curvature and derivatives of the couplings. There is only a finite number of possible basis terms and their coefficient functions 
can be perturbatively determined for a particular theory.

Wess-Zumino consistency

Without resorting to a particular theory, one may nevertheless find constraints between the coefficients arising from a Wess-Zumino consistency condition

$$
\left[\Delta_{\sigma}, \Delta_{\sigma^{\prime}}\right] W=0
$$

In two dimensions this consistency condition implies $\beta^{i} \partial_{i}\left(c+w_{i} \beta^{i}\right)=$ $\chi_{i j} \beta^{i} \beta^{j}$, where $c$ is the central charge and $w_{i}\left(\lambda^{k}\right)$ and $\chi_{i j}\left(\lambda^{k}\right)$ are above mentioned coefficient functions. $\chi_{i j}$ can be related to the positive definite Zamolodchikov metric, which is the key ingredient for the definition of a monotonic $c$-function.

failure In the four-dimensional case it is such a relation to a positive definite object that is missing. In particular the analogous consistency condition for the $a$ coefficient in the four dimensional trace anomaly $(\star)$ reads

$$
\beta^{i} \partial_{i}\left(a+\frac{1}{8} w_{i} \beta^{i}\right)=\frac{1}{8} \chi_{i j}^{g} \beta^{i} \beta^{j}
$$

where $\chi_{i j}^{g}\left(\lambda^{k}\right)$ is one of the (many) coefficients in the four-dimensional anomaly ansatz. There is a relation to a positive definite coefficient $\chi^{a}$, $\chi_{i j}^{g}=2 \chi_{i j}^{a}+$ (other terms), but it is spoiled by the occurrence of extra terms.

In supersymmetric theories, some of these extra terms are known to vanish and there might be hope that additional constraints arise from a local RG equation incorporating super-Weyl transformations that allow the construction of a monotonic $a$-function. Before tackling this ambitious task, a first step is to analyse the trace anomaly in a supersymmetric framework, which is what has been pursued in the second part of this thesis.

contents In Chapters 6 and 7 respectively, I give an introduction to minimal supergravity in an $\mathcal{N}=1$ superfield formulation and to the non-supersymmetric local renormalisation group technique outlined above.

In Chapter 8, I present superfield versions of the local RG equation, give a complete ansatz for the trace anomaly, and determine the full set of consistency equations. I then discuss the $\mathcal{N}=4$ case, which gives rise to an interesting puzzle: In [49] by a component approach a one-loop result 
for the trace anomaly of $\mathcal{N}=4$ SYM was found to contain a conformally covariant operator of fourth order, the Riegert operator [50], which is reviewed in Section 7.1.4. In [51] a supersymmetric version of this operator is given in components, but I was not able to find a satisfactory superfield version of this operator. A superfield Riegert operator is known to exist in new-minimal supergravity [52], which however in general is known to be inconsistent on the quantum level $[53,54]$. I discuss the possible origin of that problem, which I suspect to arise from the impossibility to separate local $\mathrm{U}(1)_{R}$ transformations from super-Weyl transformations in the minimal supergravity formulation such that a too strong symmetry requirement is imposed on the ansatz.* Nevertheless the extended calculations presented here should provide a good starting point for further exploration of this fascinating topic. In the conclusions possible future steps are discussed.

${ }^{*}$ In new-minimal supergravity this problem does not arise because $\mathrm{U}(1)_{R}$ is indeed a local symmetry of the theory. 



\section{Part I}

Generalizations of AdS/CFT 

"After all, all he did was string together a lot of old, well-known quotations."

H. L. Mencken, on Shakespeare

\section{Chapter 1}

\section{Overview}

$\S 1.1$ QCD, 3. $\S 1.2 \mathcal{N}=4$ Super-Yang-Mills Theory, 5. $\S 1.3$ Type IIB Supergravity, 6. $\S 1.3 .1$ p-brane Solutions, 8. $\S 1.4$ D-branes, 9. $\S 1.4 .1$ Abelian, 10. $§ 1.4 .2$ Non-Abelian, 11. §1.4.3 Quadratic Action, 13. §1.5 AdS/CFT Correspondence, 15.

\section{$1.1 \quad$ QCD}

The gauge theory of the strong interaction, quantum chromodynamics (QCD), is based on the success of the parton model [55, 56], which describes the high-energy behaviour of hadrons as bound states of localised but essentially free particles, to describe the high-energy hadron spectrum. The other key ingredient was to realise that an additional hidden threevalued quantum number, colour, is needed.

The former means that the theory should be asymptotically free; i.e. the coupling constant becomes small in the ultraviolet regime (UV). This requirement is only met by Yang-Mills theories, that means non-Abelian gauge theories.

The latter (hiding the colour) makes plausible a colour dependent force to form colour singlets only, such that one may assume the colour symmetry (as opposed to the flavour symmetry) to be gauged. Indeed lattice 


\begin{tabular}{c|c|c|c|c|}
\multicolumn{7}{|c|}{ Quark Masses } \\
Type & $Q$ & \multicolumn{3}{|c|}{ Generations } \\
\hline up & $\frac{2}{3}$ & $\mathbf{u}$ & $\mathbf{c}$ & $\mathbf{t}$ \\
\hline & & 1.5 to $4 \mathrm{MeV}$ & 1.15 to $1.35 \mathrm{GeV}$ & 169 to $179 \mathrm{GeV}$ \\
\hline down & $-\frac{1}{3}$ & $\mathbf{d}$ & $\mathbf{s}$ & $\mathbf{b}$ \\
& & 4 to $8 \mathrm{MeV}$ & 80 to $130 \mathrm{MeV}$ & 4.1 to $4.4 \mathrm{GeV}$ \\
\hline
\end{tabular}

Table 1.1: Quark masses (Particle Data Group [57])

calculations demonstrated that QCD is confining, such that the formation of colour singlets is a consequence of the dynamics.

QCD Lagrangean

The QCD Lagrangean describes an $\mathrm{SU}\left(N_{c}\right)$ Yang-Mills theory with $N_{c}=3$ the number of colours and $N_{f}=6$ the number of quarks, with a global $\mathrm{SU}\left(N_{f}\right)_{L} \times \mathrm{SU}\left(N_{f}\right)_{R} \times \mathrm{U}(1)_{V} \times \mathrm{U}(1)_{A}$ symmetry that is partly broken by the different mass of the six quarks, cf. Table 1.1. It is given by

$$
\begin{aligned}
\mathscr{L}_{\mathrm{QCD}} & =-\frac{1}{2} \operatorname{Tr} F_{m n} F^{m n}+\sum_{i}^{N_{f}} \bar{q}_{i}\left(i \gamma^{m} D_{m}-m_{i}\right) q_{i} \\
F_{m n} & =\partial_{m} A_{n}-\partial_{n} A_{m}+i g\left[A_{m}, A_{n}\right] \\
D_{m} q_{i} & =\left(\partial_{m}-i g A_{m}\right) q_{i} \\
A_{m} & =A_{m}{ }^{a} T^{q} \\
{\left[T^{a}, T^{b}\right] } & =i f^{a b c} T^{c}
\end{aligned}
$$

The $N_{c}^{2}-1=8$ fields $A_{m}{ }^{a}$ are called gluons, the $N_{f}=6$ quark fields $q_{i}$ are the Dirac fermions $u, d, s, c, b, t$. The global flavour symmetry is explicitly broken by (the inequality of) the masses $m_{i}$, though they can be assumed to be realised approximately for the isospin group $\mathrm{SU}(2)_{f}$ or even (including the strange quark) SU $(3)_{f}$. The corresponding transformation and algebra as well as Noether current and charge read

$$
\begin{array}{rlrl}
\delta q^{i} & =i \alpha^{a} t_{i j}^{a} q_{j}, & {\left[t^{a}, t^{b}\right]=i f^{a b c} t^{c},} \\
J_{\mu}^{a}=\bar{q}_{i} \gamma_{\mu} t_{i j}^{a} q_{j}, & {\left[Q^{a}, Q^{b}\right]=i f^{a b c} Q^{c},} \\
Q^{a}=\int d^{3} x J_{0}^{a}, &
\end{array}
$$


where for $S U(3)_{f}$ the generators $t^{a}=\frac{\lambda^{a}}{2}$ are usually expressed by the eight Gell-Mann matrices $\lambda^{a}$.

Furthermore the Lagrangean is invariant under an overall $\mathrm{U}(1)_{V}$ vector axial symmetry $q \mapsto \mathrm{e}^{i \alpha} q$, often also referred to by baryon number symmetry. transformation The massless version of (1.1) is in addition invariant under the $\mathrm{U}(1)_{A}$ axial transformations $q \mapsto \mathrm{e}^{i \beta \gamma_{5}} q$ giving rise to a second copy of the flavour symmetry group,

$$
\begin{aligned}
\delta q^{i} & =i \alpha^{a} t_{i j}^{a} q_{j}, & J_{\mu}^{5 a} & =\bar{q}_{i} \gamma_{\mu} t_{i j}^{a} q_{j}, \\
Q^{5 a} & =\int d^{3} x J_{0}^{5 a}, & {\left[Q^{5 a}, Q^{5 b}\right] } & =i f^{a b c} Q^{5 c} .
\end{aligned}
$$

Together they form the chiral symmetry group $\mathrm{SU}\left(N_{f}\right)_{L} \times \mathrm{SU}\left(N_{f}\right)_{R}$, whose generators and corresponding algebra are given by

$$
\begin{aligned}
Q_{L}^{a} & =\frac{1}{2}\left(Q^{a}-Q^{5 a}\right), & Q_{R}^{a} & =\frac{1}{2}\left(Q^{a}+Q^{5 a}\right), \\
{\left[Q_{L}^{a}, Q_{L}^{b}\right] } & =i f^{a b c} Q_{L}^{c}, & {\left[Q_{R}^{a}, Q_{R}^{b}\right] } & =i f^{a b c} Q_{R}^{c}, \\
{\left[Q_{L}^{a}, Q_{R}^{b}\right] } & =0 . & &
\end{aligned}
$$

When switching on mass terms this symmetry is not exact anymore and the associated charges, while still obeying the algebra, are not conserved; i.e. become time dependent.

\section{$1.2 \mathcal{N}=4$ Super-Yang-Mills Theory}

While classically Yang-Mills theories are conformally invariant, this is no longer true upon quantisation and the conformal symmetry becomes anomalous. It turns out that it is actually quite hard to find a field theory that is conformally invariant on the quantum level and it comes as a surprise that $\mathcal{N}=4 \mathrm{SYM}$, whose formulation was first achieved by compactifying ten dimensional $\mathcal{N}=1 \mathrm{SYM}$ on a six dimensional torus, actually preserves a larger symmetry group than its higher dimensional ancestor and has vanishing $\beta$ functions to all orders in perturbation theory [58].

Consequently from the commutators of supercharges and the generator of special conformal transformation, an additional set of (so-called confor- 
mal) supercharges is generated. From the perspective of AdS/CFT correspondence this doubling of supercharges is quite important since $\mathcal{N}=4$ has therefore the same number of supercharges as five dimensional maximally supersymmetric supergravity. The full superconformal algebra is $\mathrm{SU}(2,2 \mid 4)$, where its bosonic subgroups are $\mathrm{SU}(2,2) \simeq \mathrm{SO}(2,4)$, the conformal group in four dimensions, and $\mathrm{SU}(4)_{\mathrm{R}}$, the R-symmetry group.

multiplets

Being maximally supersymmetric, $\mathcal{N}=4$ SYM consists entirely of one multiplet, the $\mathcal{N}=4$ gauge multiplet. In $\mathcal{N}=1$ language, this corresponds to one gauge multiplet plus three chiral multiplets.* So the field content is one vector, four chiral fermions and three complex scalars. As the gauge and SUSY generators commute, all fields are in the adjoint representation. Two of the chiral superfields form an $\mathcal{N}=2$ hypermultiplet, while the other chiral superfield together with the $\mathcal{N}=1$ gauge multiplet forms an $\mathcal{N}=2$ gauge multiplet.

Lagrangean $\quad$ In $\mathcal{N}=1$ superfield language the Lagrangean reads

$$
\mathscr{L}=\int d^{4} \theta \operatorname{Tr}\left(\bar{\Phi}^{i} \mathrm{e}^{2 V} \Phi^{i} \mathrm{e}^{-2 V}\right)+\left[\frac{1}{4 g^{2}} \int d^{2} \theta W_{\alpha} W^{\alpha}+\int d^{2} \theta W+\text { c.c. }\right],
$$

where the gauge field strength is given by $W_{\alpha}=-\frac{1}{8} \bar{D}^{2}\left(\mathrm{e}^{-2 V} D_{\alpha} \mathrm{e}^{2 V}\right)$ and the superpotential is

$$
W=\operatorname{Tr} \Phi^{3}\left[\Phi^{1}, \Phi^{2}\right]
$$

\subsection{Type IIB Supergravity}

There are only two maximally supersymmetric supergravity theories in ten dimensions, called type IIA and type IIB. Both are $N=2$ SUGRAs and contain (among others) two chiral gravitini, but IIA is non-chiral in the sense that these fermions have opposite chirality while IIB has gravitini of the same chirality. The particle content of the latter is given by Table 1.2.

*In an attempt to embrace both naming conventions used in SUSY, multiplets are denoted chiral, gauge or hyper in conjunction with the number of supersymmetries. Super fields on the other hand shall always mean $\mathcal{N}=1$ language and will be distinguished by their constraint (none, chiral, real, linear) and transformation behaviour of the lowest component (scalar, spinor, vector, tensor, density). 


\begin{tabular}{|c|c|c|}
\hline \multicolumn{3}{|c|}{ IIB SUGRA Particle Content } \\
\hline Symbol & \#DOF & Field \\
\hline$G_{A B}$ & $35_{B}$ & metric - graviton \\
\hline$C+i \varphi$ & $2{ }_{B}$ & axion - dilaton \\
\hline$B_{A B}+i C_{2 A B}$ & $56_{B}$ & rank 2 antisymmetric \\
\hline$C_{4 A B C D}$ & $35_{B}$ & antisymmetric rank 4 \\
\hline$\psi_{A \alpha}^{1,2}$ & $112_{F}$ & two Majorana-Weyl gravitini \\
\hline$\lambda_{\alpha}^{1,2}$ & $16_{F}$ & two Majorana-Weyl dilatini \\
\hline
\end{tabular}

Table 1.2: IIB SUGRA Particle Content [59]

IIB contains a self-dual five-form field $\tilde{F}_{5}:=F_{5}-\frac{1}{2} C_{2} \wedge H_{3}+\frac{1}{2} B \wedge F$, $F_{5}:=d C_{4}$, which makes it hard to write down an action from which all equations of motion may be derived.*

Often in the literature $[59,62]$, the following action is used, ${ }^{* *}$ augmented by the self-duality condition $\tilde{F}_{5}={ }^{*} \tilde{F}_{5}$, which has to be imposed additionally on the equations of motion and where ${ }^{*}$ denotes the Hodge dual.

IIB action

$$
\begin{aligned}
S_{\mathrm{IIB}}= & \frac{1}{2 \kappa^{2}} \int d^{10} x \sqrt{G_{\mathrm{E}}}\left\{R_{\mathrm{E}}-\frac{\partial_{A} \bar{\tau} \partial^{A} \tau}{2(\operatorname{Im} \tau)^{2}}-\frac{1}{4}\left|F_{1}\right|^{2}-\frac{1}{2}\left|G_{3}\right|^{2}-\frac{1}{4}\left|\tilde{F}_{5}\right|^{2}\right\} \\
& -\frac{1}{4 i \kappa^{2}} \int C_{4} \wedge \bar{G}_{3} \wedge G_{3}
\end{aligned}
$$

where the expressions in order of appearance are the determinant of the metric, the Ricci scalar $R_{E}$, axion-dilaton field $\tau:=C+i \mathrm{e}^{-\varphi}$ composed of the axion $C$ and the dilaton $\varphi$, field strength $F_{1}:=d C$ and $G_{3}:=$ $\sqrt{\operatorname{Im} \tau}\left(F_{3}-i H_{3}\right)$ with $F_{3}:=d C_{2}$ and $H_{3}=d B$. The complex objects have been introduced to make manifest an additional rigid $\mathrm{SL}(2, \mathbb{R})$ symmetry $\mathrm{SL}(2, \mathbb{R})$

*See $[60,61]$ for recent attempts to improve this situation.

** The conventions employed here are:

$A_{p}=\frac{1}{p !} A_{A_{1} \ldots A_{p}},\left(d A_{p+1}\right)_{A_{1} \ldots A_{p+1}}=(p+1) \partial_{\left[A_{1}\right.} A_{\left.A_{2} \ldots A_{p+1}\right]}$, and $\left|F_{p}\right|^{2}=\frac{1}{p !} F_{A_{1} \ldots A_{p}} F^{A_{1} \ldots A_{p}}$. 
of type IIB SUGRA, which transforms

$$
\begin{aligned}
\tau & \mapsto \frac{a \tau+b}{c \tau+d}, & \operatorname{det}\left(\begin{array}{ll}
a & b \\
c & d
\end{array}\right)=1, \\
G_{3} & \mapsto \frac{c \bar{\tau}+d}{|c \tau+d|} G_{3}, &
\end{aligned}
$$

and leaves invariant the other fields.

equations of motion

Many also prefer to follow the historic approach [63-67] of writing down the equations of motion only, which restricted to the graviton, axion, dilaton, and four-form Ramond-Ramond potential read:

$$
\begin{aligned}
R_{A B}= & \mathrm{e}^{2 \varphi} \partial_{A} C \partial_{B} C+\partial_{A} \varphi \partial_{B} \varphi \\
& +\frac{1}{2 \cdot 4 !} \tilde{F}_{A C_{2} \ldots C_{5}} \tilde{F}_{B} C_{2} \ldots C_{5} \\
\nabla_{A} \nabla^{A} C= & -2\left(\nabla_{A} C\right)\left(\nabla^{A} \varphi\right), \\
\nabla_{A} \nabla^{A} \varphi= & \mathrm{e}^{2 \varphi}\left(\nabla_{A} C\right)\left(\nabla^{A} C\right), \\
\partial_{\left[A_{1}\right.}\left(C_{4}\right)_{\left.A_{2} \ldots A_{5}\right]}= & \varepsilon_{A_{1} \ldots A_{5}} A_{6} \ldots A_{10} \partial_{A_{6}}\left(C_{4}\right)_{A_{7} \ldots A_{10}},
\end{aligned}
$$

where by convention the total anti-symmetric Levi-Civita symbol takes values $\pm \sqrt{-\operatorname{det} G_{E}}$ for all indices lowered (and accordingly $\pm{\sqrt{-\operatorname{det} G_{E}}}^{-1}$ for all indices raised).

\subsection{1 $p$-brane Solutions}

There is a particular class of solutions to the supergravity equations of motion (1.11) that preserve half of the supersymmetry and the subgroup $\mathrm{SO}(1, p) \times \mathrm{SO}(9-p)$ of the ten dimensional Lorentz group. Additionally they have a non-trivial $C_{p+1}$ charge coupled to the supergravity action by

$$
S_{p} \sim \int d C_{p+1}
$$

p-brane ansatz

These solutions are called $p$-branes. They are determined by the ansatz

$$
d s^{2}=H(y)^{\alpha} \eta_{\mu \nu} d x^{\mu} d x^{\nu}+H(y)^{\beta}\left(d y^{2}+y^{2} d \Omega_{5}^{2}\right)
$$

with $\eta_{\mu \nu}$ the $(p+1)$-dimensional Minkowski metric, $d \Omega_{8-p}^{2}$ the line element of the $(8-p)$-dimensional unit sphere and constants $\alpha, \beta$ to be determined 
by the equations of motion. The directions $x$ are referred to as worldvolume or longitudinal coordinates, while $y$ are called transversal.

Since to this thesis, the most relevant $p$-branes are 3-branes, their full 3 -brane solution solution in terms of bosonic supergravity fields is given,

$$
\begin{array}{rlrl}
d s^{2} & =H(y)^{-1 / 2} \eta_{\mu \nu} d x^{\mu} d x^{\nu}+H(y)^{1 / 2}\left(d y^{2}+y^{2} d \Omega_{8-p}^{2}\right), \\
\Phi & =\Phi_{0}=\mathrm{const}, & C=\mathrm{const}, \\
B_{A B} & =C_{2, A B}=0, & \\
C_{4} & =H(y)^{-1} d x^{0} \wedge \cdots \wedge d x^{3}, & \\
H(y) & =1+\sum_{i} \frac{L^{4}}{\left|\vec{y}-\vec{y}_{i}\right|}, & L^{4}=4 \pi g_{s} N \alpha^{\prime 2},
\end{array}
$$

for a distribution of 3-branes at positions $y_{i}$. Close to the origin of a single brane $\left|\vec{y}-\vec{y}_{i}\right| \ll L^{4}$, the 1 in the warp factor can be neglected such that near-horizon the geometry becomes approximately $\mathrm{AdS}_{5} \times \mathrm{S}^{5}$.

geometry

\subsection{D-branes}

A D $p$-brane is a $(p+1)$-dimensional hypersurface in the target space of string theory, where open strings can end $[68,69]$. Their discovery integrates some features of superstring theory and supergravity that would have been puzzling without them. Firstly, the open string admits two boundary kinds of boundary conditions, conditions

$$
\begin{aligned}
& \text { Dirichlet } & X^{i}(\tau, \sigma) & =\text { const } \\
& \text { Neumann } & \partial_{\sigma} X^{i}(\tau, \sigma) & =0 .
\end{aligned}
$$

However from a naïve point of view, Dirichlet boundary conditions have to be considered unphysical as they break Lorentz invariance andworse - make the open strings loose momentum trough their endpoints. With the discovery of T-duality [70-73] it became apparent that one could transform from one kind of boundary condition to the other and it was no longer possible to exclude Dirichlet boundary conditions a priori. In the D-brane picture, momentum conservation can be restored by assuming the D-branes as dynamical objects can absorb the above mentioned 


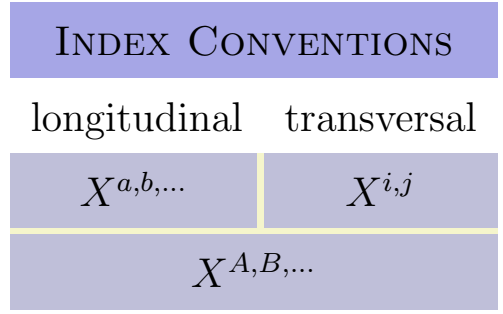

Table 1.3: Index conventions for ambient space, world volume and transversal coordinates

momentum flow.

Secondly, $p$-brane solutions* of SUGRA are interpreted as the low energy effective objects corresponding to $\mathrm{D} p$-branes.

Thirdly, it was realised early [74], that it is possible to attach gauge group factors to the end points of open strings. These Chan-Paton factors have a natural explanation as encoding which brane in a stack of coincident branes the string is attached to.

\subsubsection{Abelian}

For a single $\mathrm{D} p$-brane this factor is a $\mathrm{U}(1)$ in accordance with the fact, that the massless modes of open string theory form a $(p+1)$-dimensional U(1) SYM with one vector, $9-p$ real scalars, whose VEVs describe the position of the brane, and fermionic superpartners, which shall be ignored in the following. For constant field strengths $F_{a b}, F=\frac{1}{2} F_{a b} d X^{a} \wedge d X^{b}$, by resummation it is possible to determine the action to all orders in $\alpha^{\prime}$ [75] Dirac-Born-Infeld to be the first (Dirac-Born-Infeld, DBI) part of

$$
\begin{aligned}
S_{\mathrm{D} p}= & -T_{p} \int d^{p+1} \xi \mathrm{e}^{-\varphi} \sqrt{-\operatorname{det} P[G+B]_{a b}+2 \pi \alpha^{\prime} F_{a b}} \\
& \pm T_{p} \int P\left[\sum C_{n} \mathrm{e}^{B}\right] \mathrm{e}^{2 \pi \alpha^{\prime} F}
\end{aligned}
$$

which couples the brane to the massless Neveu-Schwarz (NS) sector of closed string theory while the second (Wess-Zumino, WZ) part determines the coupling of the brane to the massless Ramond-Ramond (RR) sector. The index conventions are depicted in Table 1.3, while the fields are explained in Section 1.3.

${ }^{*} p$-branes are domain wall solutions of SUGRA, see Section 1.3.1 for details. 
The prefactor $T_{p}$ is given by

$$
T_{p}=\frac{2 \pi}{g_{s}\left(2 \pi \ell_{s}\right)^{p+1}}
$$

with $g_{s}$ the string coupling and $\ell_{s}$ the string length.

Throughout this thesis, for explicit calculations the Kalb-Ramond field will be assumed to vanish. As will be commented on below, the Wess-Zumino term allows coupling to - with respect to the brane's world volume - lower dimensional RR potentials if the gauge field has a nontrivial Chern class. The only RR potential in the backgrounds discussed here, will be $C_{4}$ associated to the five-form flux always present in the AdS/CFT correspondence. In the particular case of a D7-brane, the WessZumino term then reads

$$
S_{D 7-W Z}=T_{p} \int d^{8} \xi P\left[C_{4}\right] \wedge F \wedge F .
$$

\subsubsection{Non-Abelian}

$N$ parallel D-branes describe a $\mathrm{U}(1)^{N}$ gauge theory. When these branes approach one another, strings stretched between different branes become light and the gauge symmetry is promoted to $\mathrm{U}(N)$. Generalising to the case of $\mathrm{U}(N)$ is straight forward in the case of D9-branes, ${ }^{*}$ which does not require a generalised pull-back and thus requires merely an additional trace over gauge indices. The action of $\mathrm{D} p$-branes of arbitrary world volume dimension $p+1$ can then be determined by T-duality, which transforms the T-dualized direction from longitudinal to transversal and vice versa. The result [76] in string frame is

non-Abelian

pull-back

$$
\begin{aligned}
S_{\mathrm{D} p}= & -T_{p} \int d^{p+1} \xi \mathrm{S} \operatorname{Tr}\left[\mathrm{e}^{-\varphi} \sqrt{\operatorname{det} Q} \sqrt{-\operatorname{det} P[\tilde{E}]_{a b}+2 \pi \alpha^{\prime} F_{a b}}\right] \\
& \pm T_{p} \int \mathrm{S} \operatorname{STr}\left[P\left[\mathrm{e}^{i\left(2 \pi \alpha^{\prime}\right) \mathrm{i}_{\Phi} \mathrm{i}_{\Phi}} \sum C_{n} \mathrm{e}^{B}\right] \mathrm{e}^{2 \pi \alpha^{\prime} F}\right]
\end{aligned}
$$

where "STr" is a trace operation that shall also take care of any ordering ambiguities in the expansion of the non-linear action. Its name ("sym-

* Apart from the additional complication of finding the correct series expansion, which is non-trivial due to ordering ambiguities. 
metrised trace") is reminiscent of an ordering prescription suggested by [77], which however is not valid beyond fifth order. Throughout this thesis, an expansion to second order will be sufficient and no ordering ambiguities appear at all.

The following abbreviations have been introduced:

$$
\begin{aligned}
\tilde{E}_{A B} & :=E_{A B}+E_{A i}\left(Q^{-1}-\delta\right)^{i j} E_{j B} \\
E_{A B} & :=G_{A B}+B_{A B}, \\
Q^{i}{ }_{j} & :=\delta_{j}^{i}+i \gamma\left[\Phi^{i}, \Phi^{k}\right] E_{k j}, \\
\left(Q^{-1}-\delta\right)^{i j} & :=\left[\left(Q^{-1}\right)^{i}{ }_{k}-\delta_{k}^{i}\right] E^{k j}, \\
\gamma & :=2 \pi \alpha^{\prime}, \\
\mathrm{i}_{\Phi} \mathrm{i}_{\Phi} f^{(n)} & :=\frac{1}{2(n-2) !}\left[\Phi^{i}, \Phi^{j}\right] f_{j i A_{3} \ldots A_{n}}^{(n)} d x^{A_{3}} \wedge \cdots \wedge d x^{A_{n}},
\end{aligned}
$$

where $f^{(n)}$ is an arbitrary $n$-form field acted upon by $\mathrm{i}_{\Phi}$, the interior product with $\Phi^{i}$. $E^{i j}$ is the inverse of $E_{i j}$ (as opposed to the transversal components of $\left.E^{A B}\right)$.

In particular static gauge is chosen,

$$
X^{a}=\xi^{a}, \quad X^{i}=\gamma \Phi^{i}\left(\xi^{a}\right),
$$

which means transversal coordinates $X^{i}$ are in one-to-one correspondence to the scalar fields $\Phi^{i}$. Then the pull-back of an arbitrary ambient space tensor $T_{A_{1} \ldots A_{n}}$ can recursively be defined by

$$
P\left[T_{A_{1} \ldots A_{n}}\right]_{a_{1} \ldots a_{n}}:=P\left[T_{a_{1} A_{2} \ldots A_{n}}\right]_{a_{2} \ldots a_{n}}+\gamma\left(\mathcal{D}_{a_{1}} \Phi^{i}\right) P\left[T_{i A_{2} \ldots A_{n}}\right]_{a_{2} \ldots a_{n}}
$$

which yields for the combined metric/Kalb-Ramond field

$$
P[\tilde{E}]:=\tilde{E}_{a b}+\gamma \tilde{E}_{a i} \mathcal{D}_{b} \Phi^{i}+\gamma \tilde{E}_{i b} \mathcal{D}_{a} \Phi^{i}+\gamma^{2} \tilde{E}_{i j} \mathcal{D}_{a} \Phi^{i} \mathcal{D}_{b} \Phi^{j}
$$

$\mathcal{D}_{a}$ denotes the gauge covariant derivative.

Finally $E_{a b}$ still may contain a functional dependence on the noncommutative scalars $\Phi$ and is to be understood as being defined by a Taylor expansion non-Abelian Taylor expansion [78] 


$$
E_{a b}\left(\xi^{a}\right)=\left.\exp \left[\gamma \Phi^{i} \partial_{X^{i}}\right] E_{a b}\left(\xi^{a}, X^{i}\right)\right|_{X^{i}=0}
$$

Again the Wess-Zumino part shall be given for the eight dimensional case; i.e. a stack of D7-branes,

$$
\begin{aligned}
S_{W Z}=T_{7} \int \operatorname{STr}\{ & P\left[C_{8}\right]+\gamma P\left[i \mathrm{i}_{\Phi} \mathrm{i}_{\Phi} C_{8}+C_{6}\right] \wedge F \\
& +\frac{\gamma^{2}}{2} P\left[\left(i \gamma \mathrm{i}_{\Phi} \mathrm{i}_{\Phi}\right)^{2} C_{8}+i \gamma \mathrm{i}_{\Phi} \mathrm{i}_{\Phi} C_{6}+C_{4}\right] \wedge F \wedge F \\
& +\frac{\gamma^{3}}{3 !} P\left[\left(i \gamma \mathrm{i}_{\Phi} \mathrm{i}_{\Phi}\right)^{3} C_{8}+\left(i \gamma \mathrm{i}_{\Phi} \mathrm{i}_{\Phi}\right)^{2} C_{6}\right. \\
& \left.\left.+i \gamma \mathrm{i}_{\Phi} \mathrm{i}_{\Phi} C_{4}+C_{2}\right] \wedge F \wedge F \wedge F\right\},
\end{aligned}
$$

where $B$ has been assumed to vanish. For a 3 -brane background, there is only a four-form potential and accordingly the Wess-Zumino part is given by

$$
S_{W Z}=T_{7} \int \mathrm{S} \operatorname{Tr} \frac{\gamma^{2}}{2} P\left[C_{4}\right] \wedge F \wedge F+\frac{i \gamma^{4}}{3 !} P\left[\mathrm{i}_{\Phi} \mathrm{i}_{\Phi} C_{4}\right] \wedge F \wedge F \wedge F
$$

While (1.18) encodes the high non-linearity of a D-brane action in a compact manner, it is often not suited for explicit calculations and needs to be expanded.

\subsubsection{Quadratic Action}

As both the non-Abelian scalars and the field strength carry $\gamma$ as a prefactor, it is tempting to think of it as an expansion parameter, keeping track of the order. However in equation (1.19c) in front of the commutator there is a factor of $\gamma$ where following this logic a factor of $\gamma^{2}$ should be expected.*

To avoid these pitfalls and unambiguously define what is meant by quadratic order "quadratic order", a parameter $\varepsilon$ shall be thought to accompany $\gamma$ in each of the equations of the last Section with the sole exception of (1.19c), where an $\varepsilon^{2}$ is included in front of the commutator. Then, the order $\varepsilon^{n}$

${ }^{*}$ Furthermore some authors prefer to use factors of $\alpha^{\prime}$ to obtain D3-transversal coordinates with mass dimension 1, thus modifying the manifest $\alpha^{\prime}$ dependence even though in physical observables such redefinitions cancel of course. 
denotes a total of $n$ fields of $\Phi$ or $F_{a b}$ in a term.

Pulling out a factor $E_{a b}(\varepsilon=0)$ (which shall also not depend on transverse directions $X^{i}$ as they come with an $\varepsilon$ ) from the DBI part of the D-brane action defines a matrix $M(\gamma)$ according to

$$
S_{\mathrm{DBI}}=-T_{p} \int d^{p+1} \xi \mathrm{STr}\left[\mathrm{e}^{-\varphi} \sqrt{\operatorname{det} Q} \sqrt{-\operatorname{det} E_{a b}(0)} \sqrt{\operatorname{det} M(\varepsilon)}\right]
$$

which has the property $M(0)=\mathbb{1}$ and is given by

$$
M(\varepsilon)_{b}^{a}=E^{a c}(\varepsilon=0)\left(P[\tilde{E}(\gamma)]_{c b}+\varepsilon \gamma F_{c b}\right)
$$

$E^{a c}$ is the inverse of $E_{a c}$. An expansion in $\varepsilon$ is performed according to

$$
\begin{aligned}
\sqrt{\operatorname{det} M(\varepsilon)}=1+\frac{\varepsilon}{2} \operatorname{Tr}\left(M^{\prime}(0)\right)+\frac{\varepsilon^{2}}{4}[ & \operatorname{Tr}\left(M^{\prime \prime}(0)\right)-\operatorname{Tr}\left(M^{\prime}(0)^{2}\right) \\
& \left.+\frac{1}{2} \operatorname{Tr}^{2}\left(M^{\prime}(0)\right)\right]+\mathcal{O}\left(\varepsilon^{3}\right)
\end{aligned}
$$

where

$$
\begin{aligned}
M^{\prime}(0)= & \gamma E^{a c} \Phi^{i} \partial_{X^{i}} E_{c b}+E^{a c}\left(\gamma E_{k b} \mathcal{D}_{c} \Phi^{k}+\gamma E_{c k} \mathcal{D}_{b} \Phi^{k}\right)+\gamma E^{a c} F_{c b} \\
M^{\prime \prime}(0)= & \gamma^{2} E^{a c} \Phi^{i} \Phi^{j} \partial_{X^{i}} \partial_{X^{j}} E_{c b} \\
& +2 \gamma^{2} E^{a c} \Phi^{i} \partial_{X^{i}}\left(E_{k b} \mathcal{D}_{c} \Phi^{k}+E_{c k} \mathcal{D}_{b} \Phi^{k}\right) \\
& +E^{a c}\left[E_{c i}\left(2 i \gamma\left[\Phi^{i}, \Phi^{j}\right]-E^{i j}\right) E_{j b}+2 \gamma^{2} E_{i j} \mathcal{D}_{c} \Phi^{i} \mathcal{D}_{b} \Phi^{j}\right]
\end{aligned}
$$

All quantities on the right hand sides of (1.29) and (1.30) are to be understood as having $\varepsilon$ set to zero. In particular this means the right hand sides are evaluated at vanishing transversal coordinates $X^{i}=0$. 
of the action up to quadratic order simplifies dramatically,

$$
\begin{aligned}
S_{\mathrm{DBI}}=-T_{p} \int d^{p+1} \xi & \operatorname{STr} \mathrm{e}^{-\varphi} \sqrt{-\operatorname{det} G_{a b}}[1+(\text { lin. }) \\
& +\frac{\gamma^{2}}{2} G^{a b} G_{i j} \mathcal{D}_{a} \Phi^{i} \mathcal{D}_{b} \Phi^{j}+\frac{\gamma^{2}}{4} G^{a c} G^{b d} F_{a b} F_{c d} \\
& \left.+\frac{\gamma^{2}}{4}\left(G^{a b} \partial_{X^{i}} \partial_{X^{j}} G_{a b}\right) \Phi^{i} \Phi^{j}\right]
\end{aligned}
$$

where the following terms vanish unless the transversal coordinates enter the metric linearly,

$$
\begin{aligned}
\text { (lin.) } & :=\frac{\gamma}{2} \operatorname{Tr} \mathcal{M}-\frac{\gamma^{2}}{4} \operatorname{Tr} \mathcal{M}^{2}+\frac{\gamma^{2}}{8} \operatorname{Tr}^{2} \mathcal{M}, \\
\mathcal{M}^{a}{ }_{c} & :=G^{a b} \Phi^{i} \partial_{X^{i}} G_{b c} .
\end{aligned}
$$

\subsection{AdS/CFT Correspondence}

The AdS/CFT correspondence (Anti-de Sitter/Conformal Field Theory) is the statement of two seemingly different theories to be equivalent. These theories are ten dimensional Type IIB string theory on an $\mathrm{AdS}_{5} \times \mathrm{S}^{5}$ spacetime background and four dimensional $\mathcal{N}=4$ extended supersymmetric $S U\left(N_{c}\right)$ Yang-Mills theory. The latter is a (super)conformal field theory with coupling constant $g_{Y M}^{2}=g_{s}$, where $g_{s}$ is the string coupling. The string theory has $N_{c}$ units of five-form flux through the $\mathrm{S}^{5}$, which is related to the equal curvature radii $L$ of the $\mathrm{AdS}_{5}$ and $\mathrm{S}^{5}$ by $L^{4}=4 \pi \ell_{s}^{4} g_{s} N_{c}$, where $\ell_{s}=\sqrt{\alpha^{\prime}}$ is the string length. This equivalence is supposed to hold for arbitrary values of $N_{c}$ and the coupling constants, but since string theory on $\mathrm{AdS}_{5} \times \mathrm{S}^{5}$ is not well-understood, it is usual to take two consecutive limits that make a supergravity description valid but still leave the duality non-trivial.

The first limit to take is the 't Hooft large $N_{c}$ limit, with $N_{c} \rightarrow \infty$ 't Hooft limit while $\lambda:=g_{Y M}^{2} N_{c}$ is kept fixed, in which the field theory reorganises itself in a topological expansion. This can be seen by using a double line representation for Feynman diagrams assigning a line to each gauge index, such that fields in the adjoint are equipped with two indices, while fields in a vector representation carry a single line. The diagrams, see Figure 1.1, then correspond to polyhedrons, which contribute with a power of $N_{c}$ that 


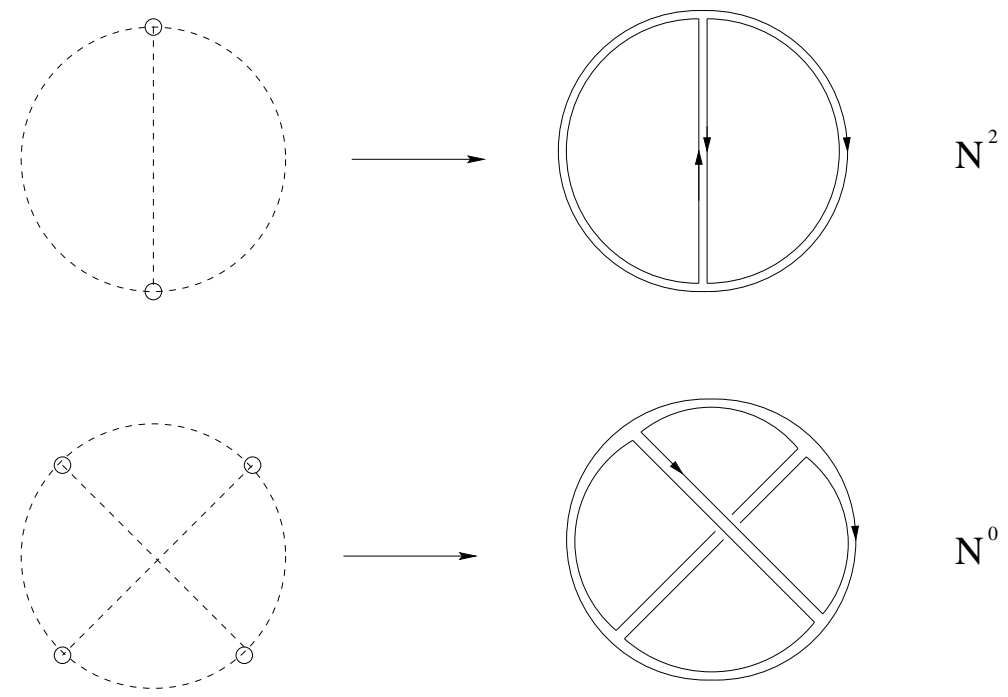

Figure 1.1: Double Line Representation: Non-planar diagrams are suppressed by powers of $N_{c}^{2}$ [79]

is suppressed by the diagram's genus and the polyhedrons are interpreted as triangulating the string world sheet, though the exact nature of this triangulation is still to be understood. Due to $g_{s}=\lambda / N_{c}$ the strict ' $t$ Hooft limit corresponds to considering classical string theory on $\operatorname{AdS}_{5} \times \mathrm{S}^{5}$. At the same time the 't Hooft coupling takes over the rôle of the field theoretic coupling constant.

small curvature

In the second limit $\ell_{s} \rightarrow 0$, the curvature radius is assumed to be large compared to the string length $\ell_{s} \ll L$. This corresponds to the low energy limit where supergravity becomes an effective description. On the field theory side this implies a large 't Hooft coupling

$$
1 \ll \frac{L^{4}}{\ell^{4}}=4 \pi \lambda
$$

and a strongly coupled theory therefore, indicating that AdS/CFT is a weakstrong duality. This means that one theory in its perturbative regime is dual to the other theory in the strong coupling regime, which renders the duality both extremely useful and hard to proof.

correlation While on the one hand the supergravity version is the weakest form of functions the AdS/CFT conjecture, it is the most useful version for practical calculations on the other hand. The equivalence of both theories to be expressed 
by

$$
\left\langle\exp \int d^{4} x \phi^{(0)} \mathcal{O}\right\rangle_{\mathrm{CFT}}=\left.\exp \left\{-S_{\mathrm{SUGRA}}[\phi]\right\}\right|_{\phi(\partial \mathrm{AdS})=\phi^{(0)}}
$$

where the field theoretic operator $\mathcal{O}$ is coupled to the boundary value $\phi_{0}$ of an associated supergravity field $\phi$, which is determined by the supergravity equations of motion and the boundary condition.

This implicitly introduces the notion of the conformal field theory be- bulk vs. ing defined on the boundary of $\mathrm{AdS}_{5}$, where one may imagine the $\mathrm{AdS}_{5}$ boundary space being build up from slices of Minkowski spaces parallel to the boundary and fibred over a fifth ("radial") direction $y$. The line element reads

$$
d s_{\mathrm{AdS}_{5} \times \mathrm{S}^{5}}=\frac{y^{2}}{L^{2}} d x_{1,3}^{2}+\frac{L^{2}}{y^{2}} d y^{2}+L^{2} d \Omega_{5}^{2} .
$$

For the metric to be invariant under rescalings of the coordinates on the boundary $x$, the radial direction has to transform reciprocal, which means that $y$ transforms as an energy and is interpreted as the renormalisation scale of the boundary theory. Considering domain wall solutions it is actually possible to represent field theoretic renormalisation group flows on the supergravity side $[10,17]$, establishing the fact that the interior of the AdS space may be interpreted as the infrared (IR) and the boundary as the ultraviolet (UV) of the field theory.

By the standard AdS/CFT dictionary supergravity fields, $\phi$ being solu- operator map tions to differential equations of second order, encode actually two field theoretic objects, whose conformal dimension can be read off from the asymptotic behaviour,

$$
\phi(y \rightarrow \infty) \sim \mathcal{J} y^{\Delta-4}+\langle\mathcal{O}\rangle y^{-\Delta},
$$

where the radial direction is interpreted as the renormalisation scale. The first, non-normalisable part corresponds to a field theoretic source and has conformal dimension $4-\Delta$; the normalisable part yields the corresponding VEV of mass dimension $\Delta$. A simple example shall illustrate this. For the bilinear operator $\bar{\psi} \psi$, the dual supergravity field has the asymptotic 
behaviour

$$
\phi(y \rightarrow \infty) \sim \frac{m}{y}+\frac{c}{y^{3}},
$$

where $m$ is the mass term of field $\psi$ and $c$ the bilinear quark condensate $\langle\bar{\psi} \psi\rangle$. The difficult part is to find out which supergravity fields correspond to which field theoretic operators. For $\frac{1}{2}$-BPS states, which correspond to superconformal chiral primary operators, the situation is simpler because they are determined by their transformational behaviour under the large global symmetry group $\mathrm{SU}(2,2 \mid 4)$. On the field theory side its bosonic subgroup $\mathrm{SO}(2,4) \times \mathrm{SU}(4) \simeq \mathrm{SO}(2,4) \times \mathrm{SO}(6)$ is realised as the conformal and R-symmetry group, while it corresponds to the isometry group on the supergravity side.

D3-branes From a string theoretical perspective, the correspondence can be understood as two different effective descriptions of a D3-brane stack, namely as a Yang-Mills theory from an open string perspective and a $p$-brane solution from a closed string perspective. In the latter case, the $\mathrm{AdS}_{5} \times \mathrm{S}^{5}$ geometry arises from a near-horizon limit. The picture of AdS/CFT being two descriptions of a D3-brane stack turns out to be particularly useful when adding additional branes to include fundamental fields into the duality. This shall be the topic of the next Chapter. 
We used to think that if we knew one, we knew two, because one and one are two. We are finding that we must learn a great deal more about "and".

Sir Arthur Eddington

\section{Chapter 2}

\section{Spicing with Flavour}

§2.1 Motivation, 20. §2.2 Probe Brane, 21. §2.3 Analytic Spectrum, 23. §2.3.1 Fluctuations of the Scalars, 23. §2.3.2 Fluctuations of the Gauge Fields, 26. $\S 2.4$ Operator Map, 28.

While the AdS/CFT correspondence has been a remarkable progress in the understanding of the 't Hooft large $N_{c}$ limit [5], a need to extend the Maldacena conjecture beyond $\mathcal{N}=4$ super-Yang-Mills (SYM) theory was soon felt, see [80] for a most prominent example. Since $\mathcal{N}=4 \mathrm{SYM}$ contains only one multiplet, the gauge field forces its representation on all other fields in the theory. As a consequence, also the fermions transform under the adjoint representation, and thus do not describe quarks.

only adjoints

There have been early attempts to augment the boundary theory with fundamental fields by including D7-branes in an $\mathrm{AdS}_{5} \times \mathrm{S}^{5} / \mathbb{Z}_{2}$ geometry $[18,19]$. The orientifold was introduced to satisfy a tadpole cancellation condition, but the dual $\mathcal{N}=2$ boundary theory had gauge group $\operatorname{Sp}(N)$. In order to obtain an $\mathrm{SU}\left(N_{c}\right)$ gauge theory for the description of large $N_{c}$ cousins of quantum chromodynamics (QCD), [20] dropped the orientifold from the setup. This was justified by the fact, that the probe D7-brane wraps a contractible $S^{3}$ cycle on the $S^{5}$ and does not lead to a tadpole, hence. In [20] it was shown that the string mode corresponding to the 
direction in which the $S^{3}$ slips from the $S^{5}$ has negative mass square, but satisfies (saturates) the Breitenlohner-Freedman bound and does not introduce an instability.

In this Chapter, the main ideas of [20] will be reviewed, before calculating the meson spectrum of a field theory dual to a more general geometry in the next Chapter.

\subsection{Motivation}

Conventional AdS/CFT correspondence can be understood as two different limits (see the introductory Chapter) of the same object, namely a stack of $N_{c}$ coincident D3-branes in string theory. The choice on which of those $N_{c}$ branes an open string may end, is reflected by the $\mathrm{SU}\left(N_{c}\right)$ symmetry of the dual field theory. The number of ways to attach both ends to the stack is $N_{c}^{2}-N_{c}$, indicating that the field describing the open string is in the adjoint representation. When including another, non-coincident brane in this setup, a string connecting it to the stack has $N_{c}$ choices and thus describes a field transforming under the vector representation of the gauge group. Another perhaps less heuristic way to understand this scenario, is to return to the 't Hooft expansion. If one takes the intuition about the field theory's reorganisation into a triangulation of the closed

world sheet string world sheet serious, then apparently, fundamental fields will provide triangulation boundaries that lead to a triangulation of the open string world sheet. In this sense, augmenting the AdS/CFT correspondence by additional branes, which exactly provide these open strings, extends the correspondence from an open-closed duality to a full string duality.

While the inclusion of D3 or D5-branes leads to fundamental fields on the boundary of AdS that are confined to a lower dimensional defect (sowhy D7 called "defect CFTs"), the addition of D7-branes provides space-time filling fields in the fundamental representation. Furthermore it breaks supersymmetry by a factor of two; from $\mathcal{N}=4$ to $\mathcal{N}=2$ on the four-dimensional field theory side by inclusion of an $\mathcal{N}=2$ fundamental hypermultiplet given rise to by the light modes of strings with one end on the D3s and one on the D7s. 


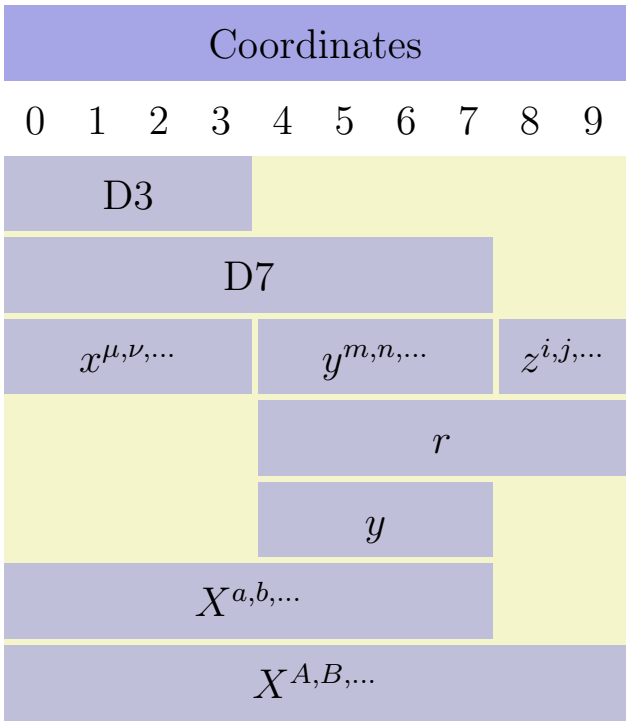

Table 2.1: D3- and D7-brane embedding in the $\mathrm{AdS}_{5} \times \mathrm{S}^{5}$ geometry. The D7-branes (asymptotically) wrap an $\mathrm{AdS}_{5} \times \mathrm{S}^{3}$. The Table also summarises the index conventions used throughout this part of the thesis.

\section{$2.2 \quad$ Probe Brane}

In order to maintain the framework of conventional AdS/CFT correspondence, [20] neglected the gravitational backreaction of the D7-branes on the geometry, which was justified by requiring the number $N_{f}$ of D7-branes to be sufficiently small. The contribution of the $N_{c}$ D3-branes and the $N_{f}$ D7-branes to the background fields is of order $g_{s}$ times their respective number. So as long as $N_{c} \gg N_{f}$, the geometry is dominated by the probe limit D3-branes and the D7-branes are approximately probe branes. In the strict $N_{c} \rightarrow \infty$ limit, which comes with the supergravity description of AdS/CFT, this approximation becomes exact.*

This is analogous to the so-called quenched approximation in lattice quenched QCD, where the action of the gauge bosons on the matter field is included, approximation while the action of the matter on the bosons is neglected.

\footnotetext{
* It should be noted that meanwhile there are supergravity solutions that include the backreaction of the D7-branes [81].
} 
The metric of $\mathrm{AdS}_{5} \times \mathrm{S}^{5}$ can be written as

$$
\begin{aligned}
d s^{2} & =\frac{r^{2}}{L^{2}} \eta_{\mu \nu} d x^{\mu} d x^{\nu}+\frac{L^{2}}{r^{2}}\left(d \vec{y}^{2}+d \vec{z}^{2}\right) \\
& =\frac{r^{2}}{L^{2}} \eta_{\mu \nu} d x^{\mu} d x^{\nu}+\frac{L^{2}}{r^{2}} d r^{2}+L^{2} d \Omega_{5}^{2},
\end{aligned}
$$

where the index conventions as well as the embedding of the D7-branes have been summarised in Table 2.1. The multiplication of vectors is supposed to denote contraction with a Euclidean metric, that means $d \vec{y}^{2}=\sum_{4,5,6,7} d y^{m} d y^{m}, d \vec{z}^{2}=\sum_{8,9} d z^{i} d z^{i}$. There are three qualitatively different types of directions: $x$ denote the world volume coordinates of the D3s, $y$ the coordinates transversal to the D3s and longitudinal to the D7s, and $z$ the coordinates transversal to both kinds of branes. Since $y$ and $z$ are on the same footing in the metric, assigning $z$ to the 8,9-plane is isometry group arbitrary, but manifestly breaks the $\mathrm{SO}(6) \simeq \mathrm{SU}(4)_{R}$ isometry group to $\mathrm{SO}(4) \times \mathrm{SO}(2) \simeq \mathrm{SU}(2)_{L} \times \mathrm{SU}(2)_{R} \times \mathrm{U}(1)_{R}$, where the orthogonal groups represent rotational invariance in the coordinates $y$ and $z$, respectively. In the case of coincident D3 and D7 branes, the hypermultiplet stemming from the strings stretched between the two stacks is massless, such that there is no classical scale introduced into the setup and conformal symmetry is maintained in the strict probe limit. Then the R-symmetry of the field theory is $\mathrm{SU}(2) \times \mathrm{U}(1)_{R}$.

embedding

When separating the stacks in the $z$-plane, the $\mathrm{SO}(2)_{8,9} \simeq \mathrm{U}(1)_{R}$ group is explicitly broken, though one may use the underlying symmetry to parametrise this breaking as

$$
z^{8}=0, \quad z^{9}=\tilde{m}_{q}
$$

Since this introduces a scale into the setup, namely a hypermultiplet mass $m_{q}=\tilde{m}_{q} /\left(2 \pi \alpha^{\prime}\right)$, it is not to be expected that conformal symmetry, and hence AdS isometry, can be maintained. The R-symmetry of the field theory becomes $\mathrm{SU}(2)_{R}$ only, which is in accordance with the geometric symmetry breaking above. 


$$
\begin{aligned}
d s^{2} & =\frac{y^{2}+\tilde{m}_{q}^{2}}{L^{2}} \eta_{\mu \nu} d x^{\mu} d x^{\nu}+\frac{L^{2} y^{2}}{y^{2}+\tilde{m}_{q}^{2}} d \vec{y}^{2} \\
& =\frac{y^{2}+\tilde{m}_{q}^{2}}{L^{2}} \eta_{\mu \nu} d x^{\mu} d x^{\nu}+\frac{L^{2}}{y^{2}+\tilde{m}_{q}^{2}} d y^{2}+\frac{L^{2} y^{2}}{y^{2}+\tilde{m}_{q}^{2}} d \Omega_{3}^{2},
\end{aligned}
$$

which towards the boundary at $|y| \rightarrow \infty$, with $y^{2} \equiv|y|^{2}:=\vec{y} \vec{y}$, approximates $\mathrm{AdS}_{5} \times \mathrm{S}^{3}$, reflecting the fact that a quark mass term is a relevant deformation that is suppressed in the ultraviolet.

This is in accordance with the usual picture of the radial direction $r=$ radius $=$ energy $\sqrt{y^{2}+\tilde{m}_{q}^{2}}$ of the AdS space describing the energy scale of the field theory, where approaching the interior of AdS from the boundary corresponds to following a renormalisation group flow from the ultraviolet (UV) to the infrared (IR).

When the renormalisation scale is lowered below the quark mass, the quarks should drop out of the dynamics. This happens when reaching the radius $r=\tilde{m}_{q}$ in the ambient space, which corresponds to the interior of the D7s at $y=0$, where the D7-branes stop from a five dimensional perspective, although as depicted in Figure 2.1 there is no boundary associated to this ending.

When $\tilde{m}_{q}=0$, the $\mathrm{U}(1)_{R}$ and $\mathrm{SO}(2,4)_{\text {AdS }}$ symmetry are restored and conformal limit the D7s fill the whole of the ambient $\mathrm{AdS}_{5}$, which suggests that conformal symmetry is restored. However, this is only true in the strict probe limit, as otherwise contributions to the beta function of order $N_{f} / N_{c}$ occur [20, 24].

\subsection{Analytic Spectrum}

\subsubsection{Fluctuations of the Scalars}

The spectrum of the undeformed D3/D7 system described above admits analytic treatment at quadratic order [24] and therefore sets the baseline for the numerical determination of meson spectra in the more complicated setups of the following Chapters.

From equations (1.14), (1.15) and (1.17) the D7-brane action in a 


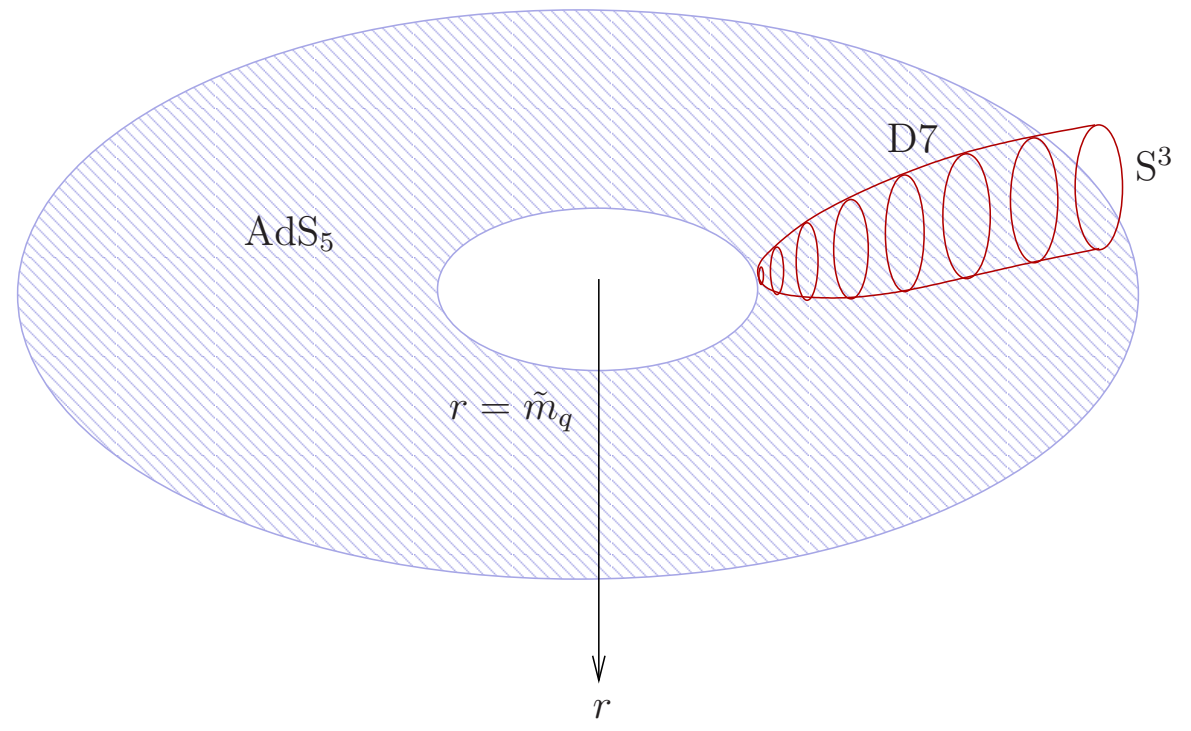

Figure 2.1: The D7-brane wraps an $S^{3}$ on the internal $S^{5}$ which slips towards a pole and shrinks to zero size. From the five dimensional point of view, the brane terminates at a certain radius, but there is no boundary associated to this ending. (Figure taken from [82])

background of D3-branes reads

$$
\begin{aligned}
S_{D 7}= & -T_{7} \int d^{8} \xi \sqrt{-\operatorname{det}\left(P[G]_{a b}+\left(2 \pi \alpha^{\prime}\right) F_{a b}\right)} \\
& +\frac{2 \pi \alpha^{\prime}}{2} T_{7} \int P\left[C_{4}\right] \wedge F \wedge F, \\
C_{4}= & \frac{r^{4}}{L^{4}} d x^{0} \wedge \cdots \wedge d x^{3},
\end{aligned}
$$

where $P$ is the pullback to the world-volume of the D7-branes and $r^{2}=$ $y^{2}+z^{2}$.

fluctuations about the embedding
For fluctuations of the scalars, the Wess-Zumino term contributes only at fourth order (with $(\text { scalar })^{2} \cdot F^{2}$ ). From the action and for an embedding according to

$$
z^{8}=0+\left(2 \pi \alpha^{\prime}\right) \delta z^{8}(\xi), \quad z^{9}=\tilde{m}_{q}+\left(2 \pi \alpha^{\prime}\right) \delta z^{9}(\xi)
$$

the expansion of the action to quadratic order (1.31) yields

$$
\mathscr{L}=\sqrt{-\operatorname{det} g_{a b}}\left(1+\frac{1}{2}\left(2 \pi \alpha^{\prime}\right)^{2} g_{i j} g^{a b} \partial_{a} z^{i} \partial_{b} z^{j}\right)
$$


where the fact that metric admits a diagonal form has been used. For the induced D7 metric (2.3), the Lagrangean (2.7) reads at quadratic order

$$
\begin{aligned}
2\left(2 \pi \alpha^{\prime}\right)^{-2} \mathscr{L} & =y^{3} \sqrt{\operatorname{det}(\hat{g})}\left[\eta^{\mu \nu}\left(\partial_{\mu} \delta z^{8}\right)\left(\partial_{\nu} \delta z^{8}\right)\right. \\
& \left.+\left(\frac{L^{2}}{y^{2}+\tilde{m}_{q}^{2}}\right)^{2}\left(\partial_{y} \delta z^{8}\right)^{2}+\hat{g}^{\alpha \beta}\left(\partial_{\alpha} z^{8}\right)\left(\partial_{\beta} z^{9}\right)+\left(z^{8} \leftrightarrow z^{9}\right)\right],
\end{aligned}
$$

with $\hat{g}_{\alpha \beta}$ the metric on the three sphere and the equation of motion

$$
\frac{L^{4}}{\left(y^{2}+\tilde{m}_{q}^{2}\right)^{2}} \partial^{\mu} \partial_{\mu} \delta z^{i}+y^{-3} \partial_{y}\left(y^{3} \partial_{y} \delta z^{i}\right)+y^{-2} \hat{\nabla}^{\alpha} \hat{\nabla}_{\alpha} \delta z^{i}=0, \quad i=8,9,
$$

equation of motion

where $\hat{\nabla}_{\alpha}$ is the covariant derivative on the unit $S^{3}$. An ansatz for sep- radial equation aration of variables $\delta z^{i}\left(x^{\mu}, y, \mathrm{~S}^{3}\right)=\zeta^{i}(y) \mathrm{e}^{i k \cdot x} \mathcal{Y}^{\ell}\left(\mathrm{S}^{3}\right)$, with $\hat{\nabla}^{\alpha} \hat{\nabla}_{\alpha} \mathcal{Y}^{\ell}=$ $-\ell(\ell+2) \mathcal{Y}^{\ell}, \ell \in \mathbb{N}_{0}$ yields

$$
\begin{gathered}
{\left[\partial_{\tilde{y}}^{2}+\frac{3}{\tilde{y}} \partial_{\tilde{y}}+\frac{\tilde{M}_{s}^{2}}{\left(1+\tilde{y}^{2}\right)^{2}}-\frac{\ell(\ell+2)}{\tilde{y}^{2}}\right] \zeta^{i}(\tilde{y})=0} \\
\tilde{y}=\frac{y}{\tilde{m}_{q}}, \quad \tilde{M}_{s}^{2}=-\frac{k^{2} L^{4}}{\tilde{m}_{q}^{2}}
\end{gathered}
$$

where a rescaling has removed all explicit scale dependencies. Requiring regularity at the origin, the radial equation (2.10) can be solved uniquely in terms of a hypergeometric function,

$$
\begin{aligned}
\zeta^{i}(y) & =\frac{y^{\ell}}{\left(y^{2}+\tilde{m}_{q}^{2}\right)^{n+\ell+1}}{ }_{2} \mathrm{~F}_{1}\left(-(n+\ell+1),-n ; \ell+2 ;-y^{2} / \tilde{m}_{q}^{2}\right), \\
M_{s}^{2} & =-k^{2}=\frac{4 \tilde{m}_{q}^{2}}{L^{4}}(n+\ell+1)(n+\ell+2),
\end{aligned}
$$

with the discretisation condition $n \in \mathbb{N}_{0}$ from normalisability. Note that the spectrum becomes degenerate in the conformal $\tilde{m}_{q} \rightarrow 0$ limit. The conformal dimension of the boundary operator dual to the solution above, can be read off from its scaling behaviour with respect to the radial coordinate. In [24] the UV behaviour is determined from (2.12), but one UV behaviour may instead simply discuss the radial equation (2.10), which for large $\tilde{y}$ 
becomes approximately

$$
\left[\partial_{\tilde{y}}^{2}+\frac{3}{\tilde{y}} \partial_{\tilde{y}}-\frac{\ell(\ell+2)}{\tilde{y}^{2}}\right] \zeta^{i}(\tilde{y})=0
$$

Its solutions are of the form $\zeta^{i}(\tilde{y})=A \tilde{y}^{\ell}+B \tilde{y}^{-\ell-2}$, which contradicts the naïve AdS/CFT expectation of $\tilde{y}^{\Delta-4}+\tilde{y}^{-\Delta}$ as can be seen from taking the sum of the exponents. This is due to the appearance of a determinant non-canonical factor $\sqrt{-\operatorname{det} g_{a b}} \sim \tilde{y}^{3}$, which imposes a non-canonical normalisation on normalisation the kinetic term. So the generic behaviour should be $\tilde{y}^{p+\Delta-4}+\tilde{y}^{p-\Delta}$ and subtracting the exponent of the non-normalisable solution, which corresponds to a field theory source, from that of the normalisable one, which corresponds to a vacuum expectation value, it can be seen that

$$
\begin{aligned}
& -(\ell+2)-\ell=(p-\Delta)-(p+\Delta-4)=-2 \Delta+4 \\
& \Longrightarrow \Delta=\ell+3 .
\end{aligned}
$$

\subsubsection{Fluctuations of the Gauge Fields}

The equations of motion for the gauge fields read

$$
\partial_{a}\left(\sqrt{-\operatorname{det} g_{c d}} F^{a b}\right)-\frac{4 \rho\left(\rho^{2}+\tilde{m}_{q}^{2}\right)}{L^{4}} \varepsilon^{b \beta \gamma} \partial_{\beta} A_{\gamma}=0
$$

with $\varepsilon^{\alpha \beta \gamma}$ taking values \pm 1 , and 0 when the free index $b$ is none of the angular $\mathrm{S}^{3}$ directions.

Expanding the equation of motion yields

$$
\begin{aligned}
& {\left[\left(g_{x x}\right)^{-1} \partial_{\mu} \partial^{\mu}+y^{-3} \partial_{y}\left(y^{3}\left(g_{y y}\right)^{-1} \partial_{y}\right)+\tilde{\nabla}_{\alpha} \tilde{\nabla}^{\alpha}\right] A_{\nu}} \\
& -\partial_{\nu}\left[\left(g_{x x}\right)^{-1} \partial_{\mu} A^{\mu}+y^{-3} \partial_{y}\left(y^{3}\left(g_{y y}\right)^{-1} A_{y}\right)+\tilde{\nabla}^{\alpha} A_{\alpha}\right]=0 \\
& {\left[\left(g_{x x}\right)^{-1} \partial_{\mu} \partial^{\mu}+\tilde{\nabla}_{\alpha} \tilde{\nabla}^{\alpha}\right] A_{y}-\partial_{y}\left[\left(g_{x x}\right)^{-1} \partial_{\mu} A^{\mu}+\tilde{\nabla}^{\alpha} A_{\alpha}\right]=0}
\end{aligned}
$$




$$
\begin{aligned}
& {\left[\left(g_{x x}\right)^{-1} \partial_{\mu} \partial^{\mu}+y^{-3} \partial_{y}\left(y^{3}\left(g_{y y}\right)^{-1} \partial_{y}\right)+\tilde{\nabla}_{\alpha} \tilde{\nabla}^{\alpha}\right] A_{\delta}} \\
& \quad-\partial_{\delta}\left[\left(g_{x x}\right)^{-1} \partial_{\mu} A^{\mu}+y^{-3} \partial_{y}\left(y^{3}\left(g_{y y}\right)^{-1} A_{y}\right)+\tilde{\nabla}^{\alpha} A_{\alpha}\right] \\
& -C_{4}^{\prime} \tilde{g}_{\delta \alpha} \varepsilon^{\alpha \beta \gamma} \partial_{\beta} A_{\gamma}=0,
\end{aligned}
$$

each of which has to be satisfied for a particular ansatz. For the components $\left(A_{\mu}, A_{y}, A_{\alpha}\right)$, the first two should transform under $\mathrm{SO}(4)_{4567}$ as scalars, while the last should transform as a vector and accordingly be built up from vector spherical harmonics. The simplest choice is $\tilde{\nabla}^{\alpha} \mathcal{Y}^{\ell}$, spherical which transforms in the $\left(\frac{\ell}{2}, \frac{\ell}{2}\right)$. The other two possibilities are $\mathcal{Y}_{\alpha}^{\ell, \pm}$, which harmonics transform in the $\left(\frac{\ell \pm 1}{2}, \frac{\ell \mp 1}{2}\right)$ and obey

$$
\begin{aligned}
\tilde{\nabla}^{2} \mathcal{Y}_{\alpha}^{\ell, \pm}-2 \delta_{\alpha}^{\beta} \mathcal{Y}_{\beta}^{\ell, \pm} & =-(\ell+1)^{2} \mathcal{Y}_{\alpha}^{\ell, \pm} \\
\varepsilon_{\alpha \beta \gamma} \tilde{\nabla}_{\beta} \mathcal{Y}_{\beta}^{\ell, \pm} & = \pm(\ell \pm 1) \mathcal{Y}_{\alpha}^{\ell, \pm}, \\
\tilde{\nabla}^{\alpha} \mathcal{Y}_{\alpha}^{\ell, \pm} & =0 .
\end{aligned}
$$

The modes containing $\mathcal{Y}^{\ell, \pm}$ should not mix with the others since they are in a different representations. The following types of solutions can be obtained:

$$
\begin{aligned}
& \text { Type I } \pm A_{\alpha}=\phi_{I}^{ \pm}(y) \mathrm{e}^{i k x} \mathcal{Y}^{\ell, \pm}, \quad A_{\mu}=A_{y}=0, \\
& \text { Type II } A_{\mu}=\xi_{\mu} \phi_{I I}(y) \mathrm{e}^{i k x} \mathcal{Y}^{\ell}, \quad A_{y}=A_{\alpha}=0, \quad k_{\mu} \xi^{\mu}=0 \text {, } \\
& \text { Type III } A_{y}=\phi_{I I I}(y) \mathrm{e}^{i k x} \mathcal{Y}^{\ell}, \quad A_{\alpha}=\tilde{\phi}_{I I I}(y) \mathrm{e}^{i k x} \tilde{\nabla}_{\alpha} \mathcal{Y}^{\ell} \text {. }
\end{aligned}
$$

Type II and III come from recognising that in the gauge $\partial_{\mu} A^{\mu}=0, A_{\mu}$ does not appear in (2.17) and (2.18), and can therefore be treated independently. Kruczenski et al. argue that modes not satisfying the gauge condition are either irregular or have a polarisation parallel to the wave vector $k$; i.e. can be brought to the gauge $\partial_{\mu} A^{\mu}=0$.

The simplest radial equation arises from the ansatz II,

$$
\left[\partial_{\tilde{y}}^{2}+\frac{3}{\tilde{y}} \partial_{\tilde{y}}+\frac{\tilde{M}_{I I}^{2}}{\left(1+\tilde{y}^{2}\right)^{2}}-\frac{\ell(\ell+2)}{\tilde{y}^{2}}\right] A_{a}=0 .
$$

Up to the polarisation vector, this is the same equation as (2.9) and therefore produces a degeneracy of the mass spectrum, 


$$
\tilde{M}_{I I}^{2}=\tilde{M}_{s}^{2}=4(n+\ell+1)(n+\ell+2), \quad n, \ell \geq 0,
$$

with the same conformal dimension $\Delta=\ell+3$.

For type III and $\mathrm{I} \pm$, an analogous calculation yields the mass formulae and conformal dimensions of the corresponding UV operators,

$$
\begin{array}{lll}
\tilde{M}_{I+}^{2}=4(n+\ell+2)(n+\ell+3), & \Delta=\ell+5 & \ell \geq 1, \\
\tilde{M}_{I-}^{2}=4(n+\ell)(n+\ell+1), & \Delta=\ell+1 & \ell \geq 1, \\
\tilde{M}_{I I I}^{2}=4(n+\ell+1)(n+\ell+2), & \Delta=\ell+3 & \ell \geq 1,
\end{array}
$$

with $n \geq 0$ in all cases.

matching of

The full mesonic mass spectrum is given in Table 2.2, were the Dirac representations fermions needed to fill the states into massive $\mathcal{N}=2$ supermultiplets have been added. Since the $\mathrm{SU}(2)_{L}$ group commutes with the supercharges, all states in the same supermultiplet should be in the same representation with respect to the left quantum number. Indeed redefining $\ell$ in such a manner that the $\mathrm{SU}(2)_{L}$ representations are the same also makes the mass coincide. This argument cannot be applied to the right quantum number, for the supercharges are not singlets under the $R$-symmetry. (Although the spectrum is symmetric under swapping the rôles of the left and right group, which corresponds to considering an anti-D7-brane, that is the opposite sign in front of the Wess-Zumino term.)

\subsection{Operator Map}

As has been seen, the fluctuation modes of the D7-brane organise themselves in $\mathcal{N}=2$ multiplets, which are made of a chiral primary field and descendants. The mode with highest $\mathrm{SU}(2)_{R}$ quantum number is the scalar of type (I-). The choice of the corresponding primary operator

lowest primary is restricted by the requirement of containing exact two hypermultiplet fields in the fundamental representation, being in the same representation $\left(\frac{\ell}{2}, \frac{\ell+2}{2}\right)_{0}$ and having conformal dimension $\Delta=\ell+2$. For $\ell=0$ this merely admits the unique combination

$$
\mathcal{O}^{I}=\psi^{\alpha} \sigma_{\alpha \dot{\beta}}^{I} \bar{\psi}^{\dot{\beta}}
$$




\begin{tabular}{|c|c|c|c|c|c|}
\hline \multicolumn{6}{|c|}{ IIB SUGRA Particle Content } \\
\hline \multicolumn{2}{|c|}{ Type } & \multirow{2}{*}{$\begin{array}{c}\mathrm{SU}(2)_{R} \\
\frac{\ell+2}{2}\end{array}$} & \multicolumn{2}{|l|}{$\mathrm{U}(1)_{R}$} & \multirow{2}{*}{$\begin{array}{c}\Delta-\ell \\
2\end{array}$} \\
\hline 1 scalar & $(\mathrm{I}-)$ & & 2 & $\ell \geq 0$ & \\
\hline 2 scalars & $(\mathrm{s})$ & $\frac{\ell}{2}$ & 0 & $\ell \geq 0$ & 3 \\
\hline 1 vector & (II) & $\frac{\ell}{2}$ & 0 & $\ell \geq 0$ & 3 \\
\hline 1 scalar & (III) & $\frac{\ell}{2}$ & 0 & $\ell \geq 1$ & 3 \\
\hline 1 scalar & $(\mathrm{I}+)$ & $\frac{\ell-2}{2}$ & 0 & $\ell \geq 2$ & 4 \\
\hline 1 Dirac & $(\mathrm{F} 1)$ & $\frac{\ell+1}{2}$ & 1 & $\ell \geq 0$ & $\frac{5}{2}$ \\
\hline 1 Dirac & (F2) & $\frac{\ell-1}{2}$ & 1 & $\ell \geq 1$ & $\frac{9}{2}$ \\
\hline
\end{tabular}

Table 2.2: Mesonic Spectrum in $\mathrm{AdS}_{5} \times \mathrm{S}^{5}$. The Dirac fermions are deduced from Supersymmetry. $\Delta$ is the conformal dimension of the corresponding UV operator and the representations have been shifted to have the same $\mathrm{SU}(2)_{L}$ spin $\frac{\ell}{2}$ and therefore the same mass $\tilde{M}^{2}=$ $4(n+\ell+1)(n+\ell+2), n \geq 0$.

with the Pauli matrices $\sigma^{I}$. The higher chiral primary in the Kaluza-Klein Kaluza-Klein tower, can be obtained by including the adjoint operators obtained a the primaries subset $Y^{4,5,6,7}$ of the six adjoint scalars of the $\mathcal{N}=4$ multiplet by traceless symmetrisation, ${ }^{*}$

$$
\chi_{\ell}=Y^{\left(i_{1}\right.}, \ldots Y^{\left.i_{\ell}\right)}
$$

The operators $\chi_{\ell}$ transforms under $\mathrm{SU}(2)_{L} \times \mathrm{SU}(2)_{R} \times \mathrm{U}(1)_{R}$ as $\left(\frac{\ell}{2}, \frac{\ell}{2}\right)_{0}$, which in the combination

$$
\mathcal{O}_{\ell}^{I}=\psi \chi_{\ell} \sigma^{I} \bar{\psi}
$$

gives a $\left(\frac{\ell}{2}, \frac{\ell+2}{2}\right)_{0}$ of conformal dimension $\Delta=\ell+2$. The other operators can be obtained from acting with supercharges on those chiral primaries.

${ }^{*}$ The four scalars belong to the $\mathcal{N}=2$ hypermultiplet. 

Where a calculator on the ENIAC is equipped with 18,000 vacuum tubes and weighs 30 tons, computers in the future may have only 1,000 vacuum tubes and perhaps weigh 1.5 tons.

unknown, "Popular Mechanics", March 1949

\section{Chapter 3}

\section{First Deformation: \\ Geometry}

$\S 3.1$ Chiral Symmetry Breaking, 31. $§ 3.2$ DBI to Quadratic Order, 35 . $§ 3.3$ Quadratic Fluctuations, 36. §3.4 Equations of Motion, 38. §3.4.1 Vacuum, 38. §3.4.2 Pseudoscalar Mesons, 38. §3.4.3 Scalar Mesons, 38. §3.4.4 Vector Mesons, 39. §3.5 Backgrounds, 39. §3.5.1 $\mathrm{AdS}_{5} \times \mathrm{S}^{5}, 39$. §3.5.2 Gubser's Geometry, 40 . $\S 3.6$ Chiral Symmetry Breaking in Gubser's Background, 42. §3.7 Mesons, 46. $\S 3.8$ Highly Excited Mesons, 48.

\subsection{Chiral Symmetry Breaking}

While much progress has been made in the sector of AdS/CFT correspondence, it has proved difficult to find a realistic holographic dual of QCD. There are many reasons, which range from practical - working with tendimensional supergravity equations - to principle: The ultraviolet (UV) regime is weakly coupled, which corresponds to strong coupling (large curvature) on the AdS side and hence the requirement of quantising string theory on that background. Furthermore models discussed so far contain only one scale and cannot provide a separation of supersymmetry (SUSY) breaking and confinement scale $\Lambda_{Q C D}$.

Despite those obstacles AdS/CFT correspondence has been remarkably 
successful in capturing many aspects of QCD. In this Chapter, such an chiral symmetry aspect will be the important feature of chiral symmetry breaking, which shall be described holographically. Since supersymmetry prohibits chiral symmetry breaking as a non-vanishing chiral VEV violates D-flatness, the background geometry has to be deformed in such a way that SUSY is broken. At the same time it is desirable not to loose contact to the well tested framework of AdS/CFT. It is therefore crucial to look at a geometry that in the ultraviolet approaches $\mathrm{AdS}_{5} \times \mathrm{S}^{5}$.

dilaton deformed

Here this will be achieved by preserving in the whole space time an backgrounds $\mathrm{SO}(1,3) \times \mathrm{SO}(6)$ isometry. There are three IIB supergravity backgrounds in the literature $[38,42,83]$, which satisfy this condition. The implications of the background by Constable-Myers [42] have been studied in [82]. Here the focus shall be on the background by Gubser.

In analogy to the undeformed case of the previous Chapter, a D7-brane embedding parallel to the D3s will be considered and its scalar and vector fluctuations be studied. By diagonalising the fields, the discussion of multiple D7-branes reduces to several identical copies of the single brane case and has therefore no impact on the mass spectrum. There is however the important difference that a D7-brane stack admits non-trivial gauge configurations such that the Wess-Zumino term $C_{4} \wedge F \wedge F$ can contribute. The effect of non-trivial $F \wedge F$ will be studied in the next Chapter, the Wess-Zumino term will be assumed to vanish for now and an Abelian Dirac-Born-Infeld action (DBI) can safely be considered therefore.

chiral condensate VS. SUSY

As has been explained in Section 1.5, the quark mass $m_{q}$ and chiral quark condensate $c$ form the source/VEV pair that is described by the UV values of scalar fields on the brane. (Which in the string picture describe the transversal position of the brane.) In the supersymmetric scenario, the only solutions that have a field theoretic interpretation require $c=0$ for all $m_{q}$. In particular, this implies that there is no chiral quark condensate in the limit $m_{q} \rightarrow 0$ and no dynamical chiral symmetry breaking, hence. Basically the problem is that in terms of geometry a chiral condensate corresponds to a brane bending outward and behaving irregular towards the interior of AdS. Since the radial direction of the AdS space corresponds to the energy scale in the field theory, such a bending means that the field theory flows to the IR and comes back as is shown ("Bad") 


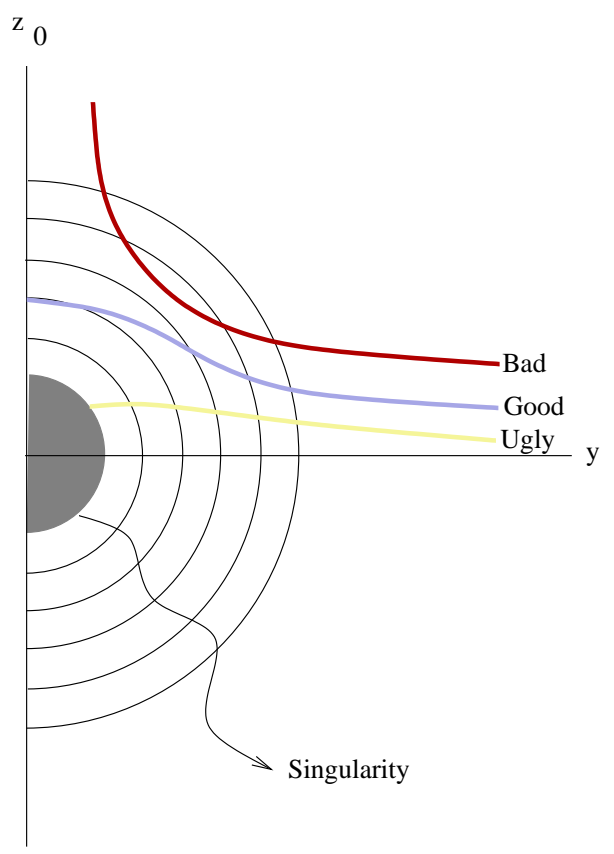

Figure 3.1: Possible solutions for the D7 embeddings. The half circles correspond to constant energy scale $\mu$. The "bad" solution cannot have an interpretation as a field theoretic flow. The "ugly" solution hits the singularity (filled circle at the centre) and can thus not be relied on. (Plot taken from [82])

in Figure 3.1. Clearly this is an unphysical behaviour. The effect of the deformed background is that the D7-brane experiences attraction from the singularity and bends inward compensating the effect of the boundary value $c$. This compensating is highly sensitive to the exact value of the chiral condensate as a function of the quark mass, which completely fixes the functional dependence.

In the previous Chapter, it was explained how adding D7-branes to the isometry AdS/CFT correspondence breaks the $\mathrm{SO}(6)_{4 \ldots 9} \simeq \mathrm{SU}(4)_{R}$ isometry of the six D3 transversal coordinates to an $\mathrm{SO}(4)_{4567} \times \mathrm{SO}(2)_{89}$ isometry, which corresponds to $\mathrm{SU}(2)_{L} \times \mathrm{SU}(2)_{R} \times \mathrm{U}(1)_{R}$, with $\mathrm{SU}(2)_{R} \times \mathrm{U}(1)_{R}$ the Rsymmetry group of the $\mathcal{N}=2$ superconformal Yang-Mills theory.* Giving a mass to the $\mathcal{N}=2$ hypermultiplet corresponds to separating the two brane stacks and breaking the conformal symmetry. This has two effects:

${ }^{*}$ To be precise, the $\mathrm{SO}(2)_{89}$ corresponds to the $\mathrm{U}(1)_{A}$ axial symmetry, while the $\mathrm{U}(1)_{R}$ R-symmetry is diag $\left[\mathrm{SO}(2)_{45} \times \mathrm{SO}(2)_{67} \times \mathrm{SO}(2)_{89}\right]$. Breaking of $\mathrm{SO}(2)_{89}$ implies breaking of the axial and R-symmetry simultaneously. 


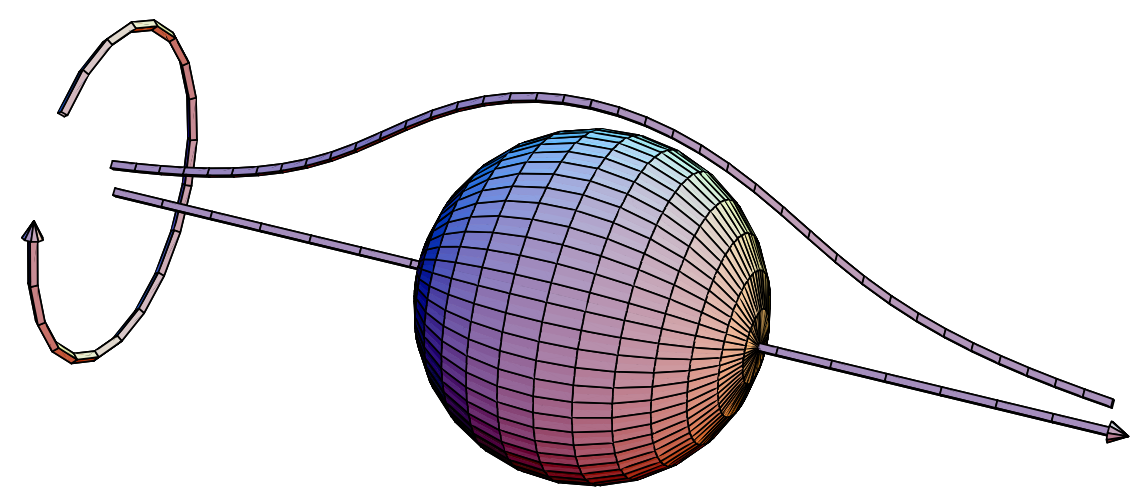

Figure 3.2: Spontaneous breaking of the $\mathrm{U}(1)_{A}$ symmetry, which rotates (circle) the 8,9-plane, by the zero quark mass solution. Zero quark mass means that the asymptotic separation between the brane embedding and the $y$-axis (large axis) vanishes. Non-vanishing quark mass means explicit and thereby not spontaneous symmetry breaking.

On the field theory side, the breaking of conformal symmetry reduces the R-symmetry to $\mathrm{SU}(2)_{R}$, on the supergravity side it breaks the rotational invariance in the 8,9-plane associated to the $\mathrm{U}(1)_{R}$. Now this breaking acquires an additional interpretation in the limit $m_{q} \rightarrow 0$, where this $\mathrm{U}(1)$ is present in the UV, but is broken dynamically by the branes bending away from the symmetry axis, cf. Figure 3.2: The symmetry spontaneously broken by the chiral condensate is the $\mathrm{U}(1)_{A}$ axial symmetry.

Since determining the chiral symmetry breaking behaviour is equivalent to finding correct D7-brane embeddings, one may go one step further and also find fluctuations about these embeddings, which corresponds to meson excitations in the correct field theoretic vacuum. For vanishing quark mass, the bilinear quark condensate breaks the axial symmetry spontaneously and the associated meson becomes massless providing a holographic version of the Goldstone theorem.

It should be noted that the explicit breaking of the $\mathrm{U}(1)_{A}$ by an instantonic anomaly, which in QCD is responsible for the $\eta^{\prime}$ to be heavy, is suppressed in the large $N_{c}$ limit. In that sense the holographic $\eta^{\prime}$ is more similar to a Pion even though it is not related to the breaking of the chiral $\mathrm{SU}\left(N_{f}\right)_{L} \times \mathrm{SU}\left(N_{f}\right)_{R}$ to its diagonal subgroup. Therefore in particular for comparison with experimental data the Pion mass is a more appropriate choice. 
This Chapter is organised as follows. First, the Dirac-Born-Infeld contents (DBI) action and the equations of motion describing the D7-brane embedding and fluctuations about the vacuum solution will be derived. Then the background by Gubser will be shortly reviewed and transformed into a convenient coordinate system. The undeformed supersymmetric scenario will be compared with a numerical evaluation of the chiral symmetry breaking and meson spectrum in the Gubser background. Additionally, the behaviour of strongly radially excited mesons will be discussed.

\subsection{DBI to Quadratic Order}

Consider the diagonal background metric

$$
d s^{2}=g_{x x}(y, z) d x_{1,3}^{2}+g_{y y}(y, z)\left(d y^{2}+y^{2} d \Omega_{3}^{2}\right)+\hat{g}_{z z}(y, z)\left(d z^{2}+z^{2} d \theta^{2}\right)
$$

which may be written as

$$
g_{(10)}=\operatorname{diag}\left(g_{x x} \mathbb{1}_{1,3}, g_{y y}, g_{y y} y^{2} \tilde{g}_{\alpha \beta}, \hat{g}_{z z}, \hat{g}_{\theta \theta}\right)
$$

where $\tilde{g}_{\alpha \beta}$ is the metric on the unit three sphere, and it holds

$$
\hat{g}_{\theta \theta}=z^{2} \hat{g}_{z z}
$$

In the case $g_{y y}=\hat{g}_{z z}$, the radial direction of the warped AdS space can be expressed as $r^{2}=y^{2}+z^{2}=\vec{y}^{2}+\vec{z}^{2}$ with $y, \Omega_{3} \mapsto y^{5}, \ldots, y^{7}$ and $z, \theta \mapsto$ $z^{8}=z \sin \theta, z^{9}=z \cos \theta$ a transformation from respectively spherical or polar to Cartesian coordinates.

Choosing static gauge,

$$
x^{0, \ldots, 3}=\xi^{0, \ldots, 3}, \quad y^{4, \ldots, 7}=\xi^{4, \ldots, 7}, \quad z^{8, \ldots, 9}=\phi^{8,9}\left(\xi^{0, \ldots, 7}\right),
$$

the DBI action in Einstein frame for a D7-brane in this background is given 
by

$$
S=\int d^{4} x d y d \Omega_{3} \sqrt{-g} \mathrm{e}^{\varphi}\left[\begin{array}{r}
1+\hat{g}_{z z} g^{a b}\left(\partial_{a} \Phi\right)\left(\partial_{b} \bar{\Phi}\right) \\
+\frac{1}{2} \mathrm{e}^{-\varphi} F_{a b} F^{a b}
\end{array}\right]^{\frac{1}{2}}
$$

where expansion to second order in the scalar fields $\Phi, \bar{\Phi}=\phi^{9} \pm i \phi^{8}$ and field strength has been performed. The remaining determinant is

$$
\sqrt{-g}=y^{3} \sqrt{\tilde{g}}\left(g_{x x} g_{y y}\right)^{2} .
$$

\subsection{Quadratic Fluctuations}

Expanding an action

$$
S=\int d^{8} \xi \mathscr{L}\left(\phi^{i}, \partial_{a} \phi^{i}\right)
$$

into small fluctuations $\delta \phi^{i}$ around a solution $\phi_{0}$ of the Euler-Lagrangeequations yields

$$
\begin{aligned}
\phi^{i} & =\phi_{0}^{i}+\varepsilon \delta \phi^{i}, \\
S & =\int d^{8} \xi \mathscr{L}_{0}+\frac{1}{2} \varepsilon^{2}\left[\frac{\partial^{2} \mathscr{L}}{\partial\left(\partial_{a} \phi^{i}\right) \partial\left(\partial_{b} \phi^{j}\right)}\right]_{\varepsilon=0}\left(\partial_{a} \delta \phi^{i}\right)\left(\partial_{b} \delta \phi^{j}\right) .
\end{aligned}
$$

Note that the above statement is merely the Legendre criterion for an extremal solution of a variational principle, which is a minimum if the parenthesised expression above is positive definite.

In accordance with the previous Chapter, where dependence on $x$ was associated to massive excitations and dependence on the spherical coordinates $\Omega_{3}$ gave rise to Kaluza-Klein states, the embedding of the D7 that forms a ground state should only depend on the radial direction $y$. For vacuum solution fluctuations about a vacuum solution $\phi_{0}=\phi_{0}(y), F_{0}^{a b} \equiv 0$, the quadratic 
expansion in scalar and vector fluctuations yields

$$
\begin{aligned}
S=\int d^{4} x d y & d \Omega_{3} \sqrt{-g} \mathrm{e}^{\varphi} \sqrt{1+\left|\phi_{0}^{\prime}(y)\right|^{2}}[ \\
1 & +\frac{1}{2}\left(\frac{g^{a b} \hat{g}_{i j}}{1+\left|\phi^{\prime}(y)\right|^{2}}\right)\left(\partial_{a} \delta \phi^{i}\right)\left(\partial_{b} \delta \phi^{j}\right) \\
& \left.-\frac{1}{2}\left(\frac{g^{y y} \hat{g}_{i j}\left(\partial_{y} \phi_{0}^{i}\right)\left(\partial_{y} \delta \phi^{j}\right)}{1+\left|\phi^{\prime}(y)\right|^{2}}\right)^{2}+\frac{1}{4} \frac{F_{a b} F^{a b}}{1+\left|\phi_{0}^{\prime}(y)\right|^{2}}\right],
\end{aligned}
$$

with

$$
\begin{aligned}
\sqrt{-g} & =y^{3}\left(g_{x x} g_{y y}\right)^{2} \sqrt{\tilde{g}}, \\
\left|\phi_{0}^{\prime}(y)\right|^{2} & :=\hat{g}_{i j} g^{a b}\left(\partial_{a} \phi_{0}^{i}\right)\left(\partial_{b} \phi_{0}^{j}\right),
\end{aligned}
$$

and $F_{a b} F^{a b}$ expressed solely in terms of fluctuations $\delta A_{m}$ about the trivial background $A_{m} \equiv 0$.

For numerics, expressing the scalar fluctuations in terms of Cartesian Cartesian vs. fields $z^{8}, z^{9}$ has some advantages. ${ }^{*}$ From the field theoretic point of view, polar expressing the fluctuations in polar coordinates $z \mathrm{e}^{i \theta}=z^{9}+i z^{8}$ is more natural, because the fluctuations of the pseudo-Goldstone mode correspond then exactly to rotations of the $\mathrm{U}(1)_{A}$. Since both approaches yield the same results due to the infinitesimal nature of the fluctuations, the polar coordinate formulation will be chosen here.

For $\Phi=z \mathrm{e}^{i \theta}, z=z_{0}(y)+\delta \sigma(\vec{x}, \vec{y})$ and $\theta=0+\delta \pi(\vec{x}, \vec{y})$, expansion of the DBI action to quadratic order in the fluctuations yields

$$
\begin{array}{rl}
S=\int d^{4} x & d y d \Omega_{3} \sqrt{\tilde{g}} \mathrm{e}^{\varphi} y^{3}\left(g_{x x} g_{y y}\right)^{2} \sqrt{1+z_{0}^{\prime}(y)^{2}}[ \\
1 & +\frac{1}{2} \frac{g^{a b} \hat{g}_{\theta \theta}\left(\partial_{a} \delta \pi\right)\left(\partial_{b} \delta \pi\right)+g^{a b} \hat{g}_{z z}\left(\partial_{a} \delta \sigma\right)\left(\partial_{b} \delta \sigma\right)}{1+z_{0}^{\prime}(y)^{2}} \\
& \left.-\frac{1}{2}\left(\frac{g^{y y} \hat{g}_{z z}\left(\partial_{y} z_{0}\right)\left(\partial_{y} \delta \sigma\right)}{1+z_{0}^{\prime}(y)^{2}}\right)^{2}+\frac{1}{4} \frac{F_{a b} F^{a b}}{1+\left|z_{0}^{\prime}(y)\right|^{2}}\right],
\end{array}
$$

where $\left(z_{0}^{\prime}\right)^{2}=\hat{g}_{z z} g^{y y}\left(\partial_{y} z_{0}\right)^{2}$.

${ }^{*}$ In particular, the excitation number $n$ of the meson tower (2.12) corresponds to the number of zeros of the solution to the radial equation (2.10), which provides a good check whether a meson solution was accidentally skipped. 


\subsection{Equations of Motion}

\subsubsection{Vacuum}

embedding From (3.12) by setting $\delta \sigma=\delta \pi=0$, the equation describing the D7 embedding in terms of $z_{0}(y)$ is obtained,

$$
\begin{aligned}
\frac{d}{d y}\left[\frac{g^{y y} g_{z z} \mathcal{F}\left(y, z_{0}\right)}{\sqrt{1+z_{0}^{\prime}(y)^{2}}} z_{0}^{\prime}(y)\right] & =g^{y y} g_{z z} \sqrt{1+z_{0}^{\prime}(y)^{2}} \frac{\partial}{\partial z_{0}} \mathcal{F}\left(y, z_{0}\right), \\
\mathcal{F} & =\mathrm{e}^{\varphi} y^{3}\left(g_{x x} g_{y y}\right)^{2} .
\end{aligned}
$$

\subsubsection{Pseudoscalar Mesons}

Goldstone mode The pseudoscalar mesons correspond to fluctuations along the $\mathrm{U}(1)_{A}$ andas shall be seen below - become massless for vanishing quark mass. They are thus (pseudo-) Goldstone bosons, which become true Goldstones for $m_{q} \rightarrow 0$. Their equations of motion are

$$
\partial_{a}\left[\frac{\sqrt{|\tilde{g}|} \mathcal{F}\left(y, z_{0}\right)}{\sqrt{1+z_{0}^{\prime}(y)^{2}}} \hat{g}_{\theta \theta} g^{a b} \partial_{b} \delta \pi\right]=0
$$

which for the ansatz $\delta \pi=\delta \pi(y) \mathrm{e}^{i k \cdot x} \mathcal{Y}^{\ell}\left(\mathrm{S}^{3}\right)$ and $M_{\pi}^{2}=-k^{2}$ read

$$
\begin{aligned}
& \frac{\sqrt{1+z_{0}^{\prime}(y)^{2}}}{\mathcal{F}} \partial_{y}\left[\mathcal{F} \frac{g^{y y} \hat{g}_{\theta \theta}}{\sqrt{1+z_{0}^{\prime}(y)^{2}}} \partial_{y} \delta \pi\right] \\
& +\left[M_{\pi}^{2} \hat{g}_{\theta \theta} g^{x x}-\ell(\ell+2) \frac{\hat{g}_{\theta \theta} g^{y y}}{y^{2}}\right] \delta \pi=0,
\end{aligned}
$$

with the same shorthand $\mathcal{F}$ as in (3.13).

\subsubsection{Scalar Mesons}

Higgs mode These correspond to fluctuations in the radial direction transverse to the 
$\mathrm{U}(1)_{A}$. The equations of motion for the scalar mesons are

$$
\partial_{a}\left[\frac{\sqrt{|\tilde{g}|} \mathcal{F}\left(y, z_{0}\right)}{\sqrt{1+z_{0}^{\prime}(y)^{2}}} \hat{g}_{z z} g^{a b} \partial_{b} \delta \sigma\right]=\partial_{y}\left[\frac{\sqrt{|\tilde{g}|} \mathcal{F}\left(y, z_{0}\right)}{{\sqrt{1+z_{0}^{\prime}(y)^{2}}}^{3}}\left(\hat{g}_{z z} g^{y y}\right)^{2}\left(\partial_{y} z_{0}\right)^{2} \partial_{y} \delta \sigma\right]
$$

which for the ansatz $\delta \sigma=\delta \sigma(y) \mathrm{e}^{i k x} \mathcal{Y}^{\ell}\left(\mathrm{S}^{3}\right)$ and $M_{\sigma}^{2}=-k^{2}$ become

$$
\begin{array}{r}
\frac{\sqrt{1+z_{0}^{\prime}(y)^{2}}}{\mathcal{F}} \partial_{y}\left[\mathcal{F} \frac{\hat{g}_{z z} g^{y y}}{\sqrt{1+z_{0}^{\prime}(y)^{2}}}\left(1-\frac{\hat{g}_{z z} g^{y y} z_{0}^{\prime}(y)^{2}}{1+z_{0}^{\prime}(y)^{2}}\right) \partial_{y} \delta \sigma\right] \\
+\left[\hat{g}_{z z} g^{x x} M_{\sigma}^{2}-\ell(\ell+2) \frac{\hat{g}_{z z} g^{y y}}{y^{2}}\right] \delta \sigma=0 .
\end{array}
$$

Again it holds $\mathcal{F}\left(y, z_{0}\right)=\mathrm{e}^{\varphi} y^{3}\left(g_{x x} g_{y y}\right)^{2}$.

\subsubsection{Vector Mesons}

In accordance with Section 2.3.2, vector mesons can be obtained from the D7-brane gauge fields whose equations of motion are

$$
\partial_{a}\left[\frac{\sqrt{\tilde{g}} y^{3}\left(g_{x x} g_{y y}\right)^{2}}{\sqrt{1+z_{0}^{\prime}(y)^{2}}} F^{a b}\right]=0
$$

for solutions with no components on the $\mathrm{S}^{3}, \delta A_{\alpha}=0$. The ansatz $\delta A_{\nu}=$ $\xi_{\nu} \delta \rho(y) \mathrm{e}^{i k \cdot x} \mathcal{Y}^{\ell}\left(\mathrm{S}^{3}\right)$, where the polarisation vector $\xi_{\nu}$ satisfies $k_{\mu} \xi_{\mu}=0$, yields

$$
\begin{aligned}
& \frac{\sqrt{1+z_{0}^{\prime}(y)^{2}}}{y^{3}\left(g_{x x} g_{y y}\right)^{2}} \partial_{y}\left[\frac{y^{3} g_{x x} g_{y y}}{\sqrt{1+z_{0}^{\prime}(y)^{2}}} \partial_{y} \delta \rho\right] \\
& +\left[\left(g^{x x}\right)^{2} M_{\rho}^{2}-\frac{\ell(\ell+2)}{y^{2}}\right] \delta \rho=0 .
\end{aligned}
$$

\subsection{Backgrounds}

\subsection{1 $\mathrm{AdS}_{5} \times \mathrm{S}^{5}$}

In this Section, it is demonstrated that the holographic description of the undeformed, supersymmetric case [24] shows no chiral symmetry breaking. To describe the field theoretic vacuum, the embedding should neither 
depend on $x$, which gives rise to a massive excitation, nor on the coordinates of the internal $\mathrm{S}^{3}$, which gives rise to Kaluza-Klein states. Using the $\mathrm{SO}(2)_{89}$ symmetry, one may choose the coordinate system such that the embedding is simply $z^{9}=z_{0}(y)$.

Then the linearised equation of motion (2.10) is given by

$$
\left[\partial_{\tilde{y}}^{2}+\frac{3}{\tilde{y}} \partial_{\tilde{y}}\right] z_{0}(y)=0
$$

with $\tilde{M}=\ell=0$. The full (as opposed to only asymptotic) solutions* are of the form

$$
z_{0}(y)=m+c y^{-2}
$$

with the conformal dimension of the dual operator $\bar{\psi} \psi$ given by $\Delta=$ $\ell+3=3$. For $c=0$, this is the constant embedding chosen by Kruczenski et al. [24] and presented in the previous Chapter. For $c \neq 0$, the solution diverges when approaching the centre $y \rightarrow 0$ of the D7-branes. This by itself is still a valid D7-brane embedding in the supergravity sense.

IR regularity However, it does not have an interpretation as a field theoretic renormalisation group flow, because the D7-brane embedding cannot be expressed as a (one-valued) function of the radial variable $r^{2}=y^{2}+z_{0}(y)^{2}$, which corresponds to the energy scale. This is also depicted as the "bad" solution in Figure 3.1.

\subsubsection{Gubser's Geometry}

A particular solution to the type IIB supergravity equations of motion (3.1) that preserves $\mathrm{SO}(1,3) \times \mathrm{SO}(6)$ isometry was found by Gubser [38], who chose an appropriate warped diagonal ansatz for the metric, a FreundRubin ansatz for the five-form flux and took only the dilaton as a nonconstant supergravity field with a radial dependence.

${ }^{*}$ Keep in mind that these are only solutions expanded to quadratic order. For the Abelian case one can do better, expand the determinant to all orders and keep the square root unexpanded. However, the outcome does not change. 
The solution presented in [38] takes the form*

$$
\begin{aligned}
d s_{10}^{2} & =\mathrm{e}^{2 \sigma} d x_{1,3}^{2}+\frac{L^{2} d \sigma^{2}}{1+B^{2} \mathrm{e}^{-8 \sigma}}+L^{2} d \Omega_{5}^{2}, \\
\varphi-\varphi_{0} & =\sqrt{\frac{3}{2}} \operatorname{arcoth} \sqrt{1+B^{-2} \mathrm{e}^{8 \sigma}},
\end{aligned}
$$

where due to $\operatorname{arcoth} x=\frac{1}{2} \ln \frac{x+1}{x-1}$ the dilaton $\varphi$ may be written as

$$
\varphi-\varphi_{0}=\sqrt{\frac{3}{8}} \ln \frac{\sqrt{1+B^{-2} \mathrm{e}^{8 \sigma}}+1}{\sqrt{1+B^{-2} \mathrm{e}^{8 \sigma}}-1} .
$$

These coordinates are such that

$$
\begin{array}{lll}
\text { IR } & \sigma \rightarrow-\infty & \text { singularity in the far interior, } \\
\text { UV } & \sigma \rightarrow+\infty & \text { boundary, }
\end{array}
$$

where there is a naked singularity in the infrared.

For calculating the meson spectrum in a background, it is more con- $\mathrm{SO}(6)$ manifest venient to work in a coordinate system that brings the metric exactly to coordinates the $\mathrm{SO}(1,3) \times \mathrm{SO}(6)$ manifest form (3.1). This can be achieved by the coordinate transformation

$$
\mathrm{e}^{2 \sigma}=\sqrt{\frac{B}{2 r_{0}^{4}}} r^{2} \sqrt{1-\frac{r_{0}^{8}}{r^{8}}}
$$

which yields

$$
\begin{aligned}
d s_{10}^{2} & =g_{x x}(r) d x_{1,3}^{2}+g_{y y}(r)\left(d r^{2}+r^{2} d \Omega_{5}^{2}\right), \\
g_{x x}(r) & =\frac{r^{2}}{L^{2}} \sqrt{1-\frac{r_{0}^{8}}{r^{8}}} \\
g_{y y}(r) & =g_{z z}=\frac{L^{2}}{r^{2}}, \\
\varphi-\varphi_{0} & =\sqrt{\frac{3}{2}} \ln \frac{r^{4}+r_{0}^{4}}{r^{4}-r_{0}^{4}} .
\end{aligned}
$$

Note that additionally $x$ has been rescaled such that $g_{x x}$ reproduces the canonical normalisation of the asymptotic AdS that is approached for $r \rightarrow$ $\infty$ and $r_{0}$ is the minimum value of $r$ where the infrared singularity resides.

${ }^{*}$ In the original publication $B^{2} / 24$ is used instead of $B^{2}$ to parametrise the deformation. 
For computations it is convenient to rescale the coordinates by $r_{0}$ such that effectively $r_{0} \mapsto 1$; i.e. all equations become independent of $r_{0}$. In this frame the quark mass is measured in units of $r_{0} T$, with $T$ the string tension, and the meson mass in units $L^{-2} r_{0}$. As will be shown below, for large quark masses the supersymmetric results of the undeformed $\mathrm{AdS}_{5} \times \mathrm{S}^{5}$ are reproduced, such that $M \sim m_{q}$. Due to

$$
\frac{M L^{2}}{r_{0}}=\text { const. } \cdot \frac{\left(2 \pi \alpha^{\prime}\right) m_{q}}{r_{0}}
$$

the supersymmetric limit $r_{0} \rightarrow 0$ allows direct identification of the numerical constant with that of equation (2.12). The situation is more complicated for the similar background of Constable-Myers, see Section 5.2.2, where by rescaling the deformation parameter cannot be entirely removed from the equations of motion, such that it also enters the numerical constant. Moreover in that background the units depend on the deformation parameter in such a way that it does not cancel in a relation similar to (3.27).

\subsection{Chiral Symmetry Breaking in Gubser's Background}

For (3.26), the equation of motion (3.13) for the vacuum solution $z=z_{0}(y)$ is given by

$$
\begin{gathered}
\frac{d}{d y}\left[\frac{y^{3} f}{\sqrt{1+z_{0}^{\prime}(y)^{2}}} z_{0}^{\prime}(y)\right]=y^{3} \sqrt{1+z_{0}^{\prime}(y)^{2}} \frac{\partial}{\partial z_{0}} f, \\
f=\frac{\left(r^{4}+1\right)^{(1+\Delta / 2)}\left(r^{4}-1\right)^{(1-\Delta / 2)}}{r^{8}}, \quad r^{2}=y^{2}+z_{0}(y)^{2}, \quad \Delta=\sqrt{6} .
\end{gathered}
$$

The constant $\Delta$ has been defined for convenient comparison to a background by Constable-Myers, cf. Chapter 5, and should not be mixed up with the conformal dimension.

Since the background (3.26) approaches $\mathrm{AdS}_{5} \times \mathrm{S}^{5}$ towards the boundary, it does not come as a surprise that the UV behaviour of $z_{0}(y)$ is given by $m_{q}+c y^{-2}$ with $m_{q}$ the quark mass and $c$ the bilinear quark conden- 


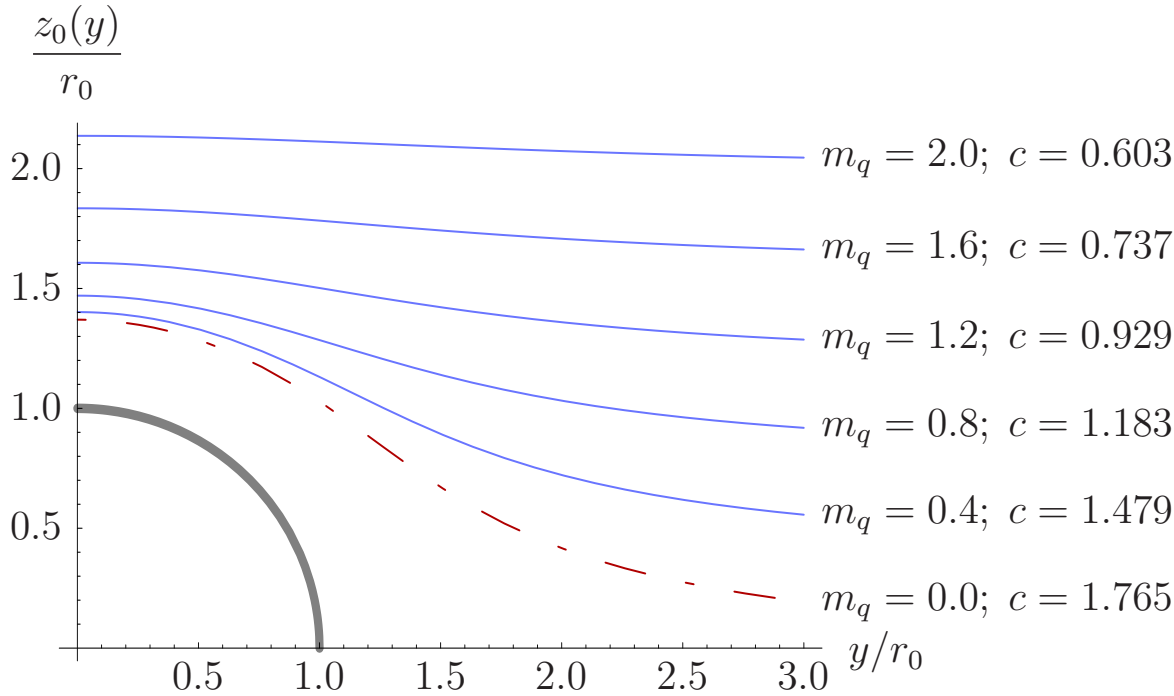

Figure 3.3: The Figure shows regular D7 embeddings with different quark mass. The embedding coordinate $z_{0}(y)$ is a radial coordinate in the 8,9-plane. While all solutions break the rotational $\mathrm{U}(1)_{A}$ symmetry in that plane, the zero quark mass solution (dashed) does so spontaneously.

sate as in the supersymmetric case. In the infrared there are still two solutions of qualitatively different behaviour: One is divergent and cannot correspond to field theoretic vacuum therefore, the other approaches a constant. However, the infrared dynamics is modified such that the pair in the UV mixes while going to the IR. Whereas in the supersymmetric case the UV solution with $c=0$ corresponded one-to-one to the regular behaviour in the IR, now for each value of $m_{q}$ there is only one value of $c$ such that the combined solution mixes into a regular one in the IR. Such regular solution have been determined numerically and are plotted in Figure 3.3. Each of the solutions is determined by a pair of quark mass and quark condensate. These pairs also determine the quark condensate as a function of the quark mass as is shown in Figure 3.4.

The possible outcomes for arbitrary combinations of $m_{q}$ and $c$ are de- qualitative picted in Figure 3.1: The solution can hit the singularity (denoted "ugly", behaviour since the supergravity approximation fails when coming to close to the singularity), the solution may diverge (denoted "bad", because it cannot correspond to a field theoretic flow), or the solution may reach a constant value for the embedding coordinate $z_{0}(y)$ at $y=0$, denoted "good". In 


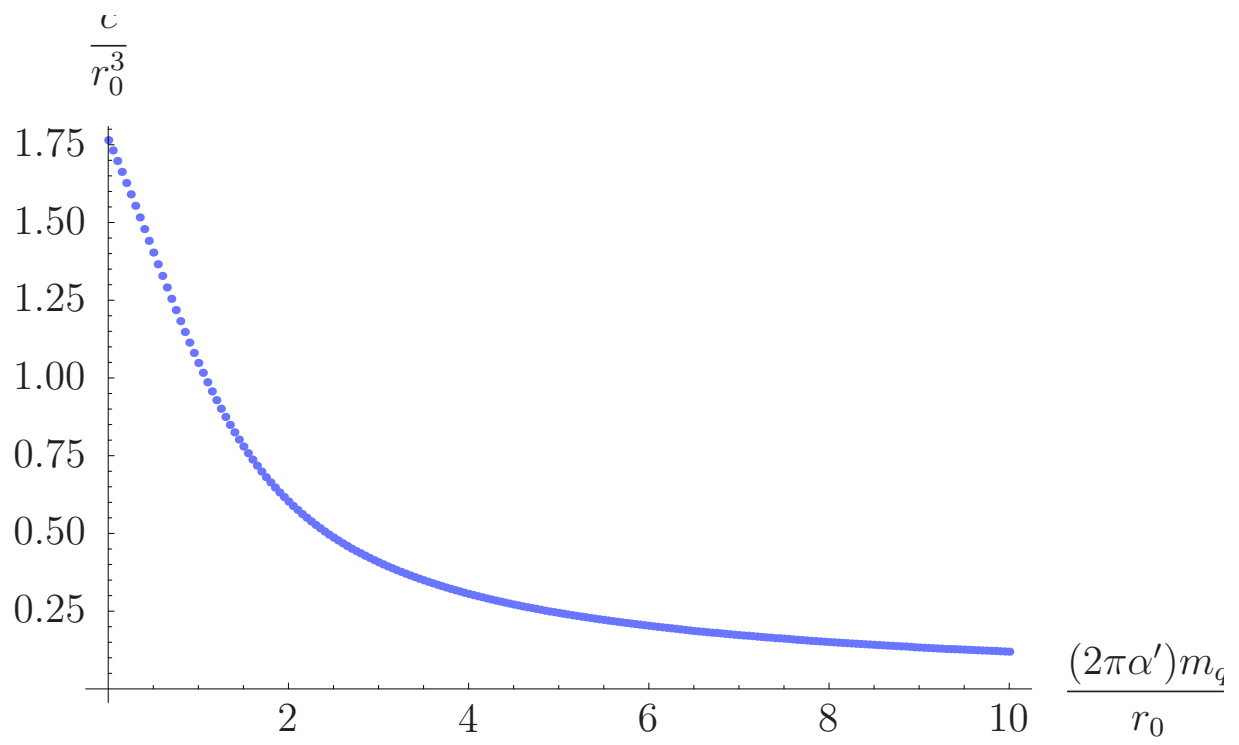

(a)

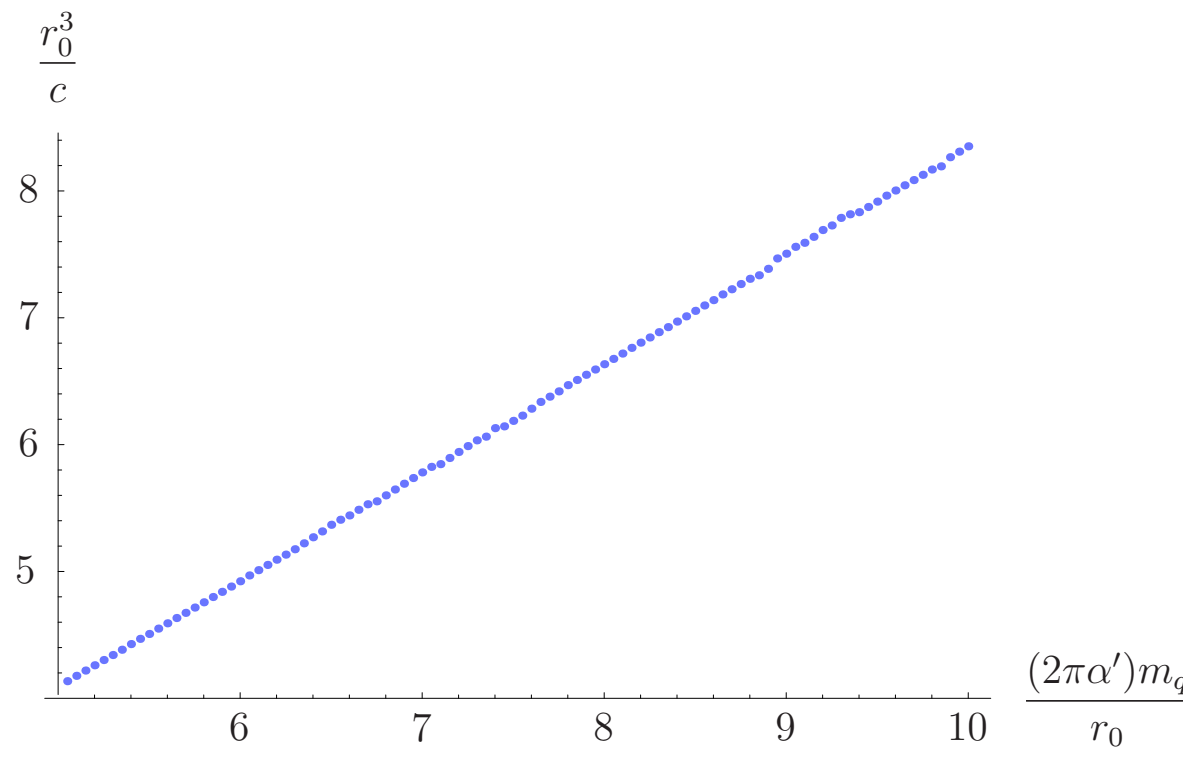

(b)

Figure 3.4: The first plot shows the chiral condensate $\langle\bar{\psi} \psi\rangle$ as a function of the quark mass $m_{q}$ as determined by regularity requirements for the D7 embedding. For large quark mass $m_{q}$ the chiral condensate behaves like $c \sim \frac{1}{m_{q}}$ in accordance with predictions from effective field theory. 
terms of the ambient space radial coordinate $r^{2}=y^{2}+z_{0}^{2}$ the D7-brane "ends" at $r=z_{0}(0)$ by the $S^{3}$ slipping from $S^{5}$ of the background and shrinking to zero size at a pole of the $\mathrm{S}^{5}$, cf. Figure 2.1. There is a stability tachyon associated to this slipping mode, but its mass obeys (saturates) the Breitenlohner-Freedman bound [21] and does not lead to an instability hence.

One might however worry about regular solutions reaching the singularity. For the discussion of whether this may happen, it is advantageous to shift the point of view to the infrared.

Starting at a finite value in the IR, there has to be a unique flow singularity to the UV, which fixes the correct combination of $m_{q}$ and $c$, since one shielding also needs the IR-divergent solution to create arbitrary combinations of $m_{q}$ and $c$. As has been explained above, $z_{0}(0)$ sets the scale were the quarks drop out of the dynamics. So one generically may expect that a large quark mass corresponds to a large value of $z_{0}(0)$. Starting at distances closer to the singularity generates solutions with smaller quark mass till one reaches a limiting solution at $z_{0}(0) \approx 1.38$ that corresponds to vanishing quarks mass. Going even closer to the singularity gives rise to a spurious negative quark mass. Due to the $\mathrm{SO}(2)_{89}$ present, these solutions are in fact positive mass solutions with negative quark condensate, as can be seen by rotating around the $y$-axis, see Figure 3.2. This assigns two potentially valid solutions to each positive quark mass. ${ }^{*}$ However solutions that do not come closer to the singularity than the zero quark mass solution have a smaller potential energy $V=-\mathscr{L}$, cf. Figure 3.6, and are therefore physical. This realises some sort of screening mechanism preventing solutions from entering the region between the zero-quark mass solution and the singularity, cf. Figure 3.5. The physical solutions outside have a positive quark condensate.

Having established the conditions that determine the chiral condensate vanishing quark as a function of the quark mass - the result is plotted in Figure 3.3- mass the case $m_{q}=0$ will be discussed in more detail now. $z_{0}(y) \equiv 0$ is obviously a solution of the equations of motion, which does however reach the singularity. To obtain a solution exhibiting chiral symmetry breaking

* The situation is to some extent analogous to asking which is the shortest route connecting two points on a sphere. The answer is a grand circle, which however also provides the longest straight route. 


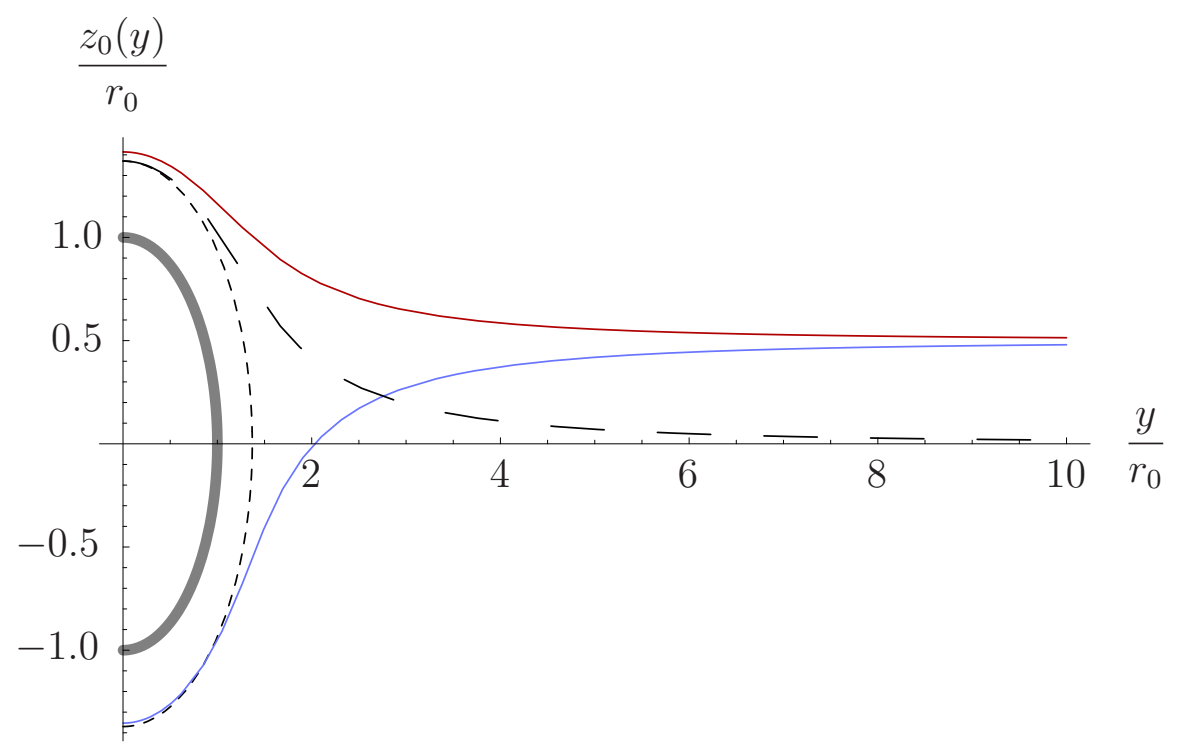

Figure 3.5: Two solutions of the same quark mass and the zero quark mass solution (dashed) are depicted. The zero mass solution exactly avoids the region between the inner circle, which is the singularity, and dashed outer "shielding" circle. Of the two massive solutions, only the one with the larger action enters the shielded region, cf. fig. 3.6.

behaviour, it is necessary to either start at a suitable value in the IR, thus forcing the solution to behave as desired, or to start with an infinitesimal deviation from $z_{0} \equiv m_{q}=0$ in the UV. This situation is analogous to calculations of the magnetisation in solid state physics, where spontaneous symmetry breaking is initiated by an arbitrarily small but non-vanishing external $B$ field.

The conclusion is that indeed spontaneous chiral symmetry breaking is observed in this geometry and one may wonder about the appearance of an associated Goldstone mode.

\subsection{Mesons}

spectrum The meson spectrum is determined by finding regular and normalisable solutions to the equations of motion arising from fluctuations about the brane embedding. These equations, given in (3.15), (3.17) and (3.19), can be solved in analogy to the case of the embedding equation (3.13), which 


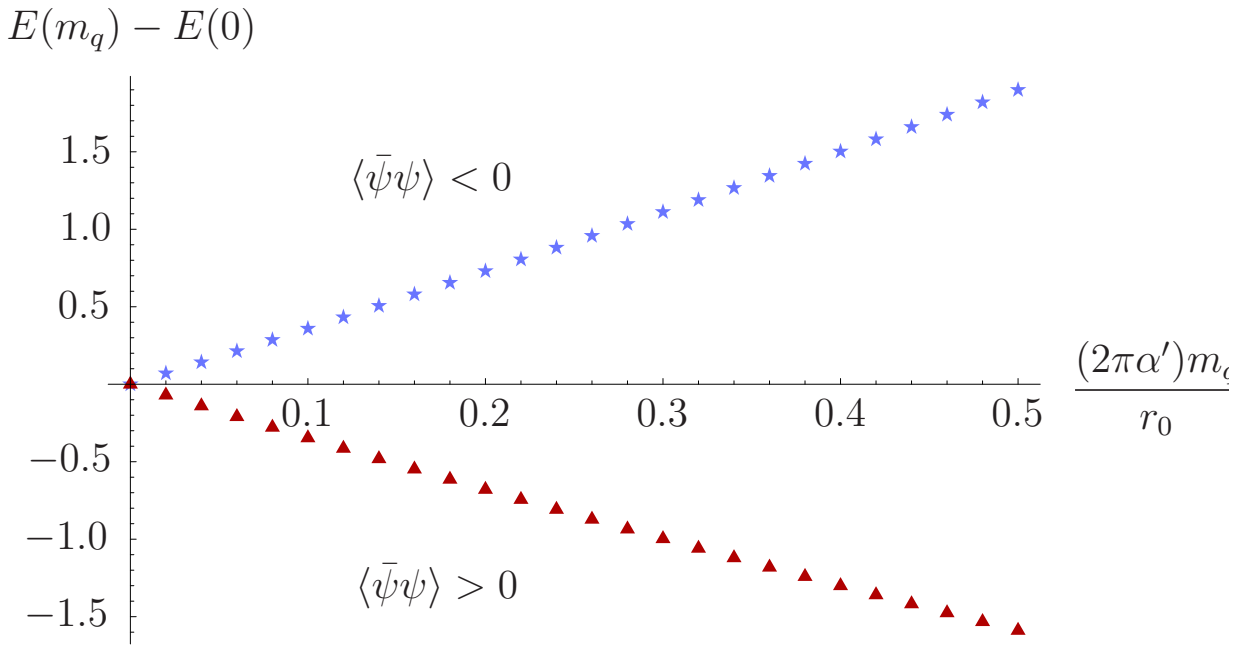

Figure 3.6: Potential Energy of D7-brane embeddings as function of the quark mass: Since the energy itself is infinite what is actually plotted is the finite difference to the action of the zero quark mass solution defined as follows,

$$
E\left(m_{q}\right)-E(0)=-\Delta S=-\lim _{Y \rightarrow \infty} \int_{0}^{Y} \mathscr{L}\left(m_{q}\right)-\mathscr{L}\left(m_{q}=0\right) d y .
$$

The physical solutions have smaller energy and are farther from the singularity than the zero-mass solution.

has been discussed in the previous Section. The solutions of the meson equations have a boundary behaviour of generic type $c_{1}+c_{2} / y^{2}$. In contrast to the embedding solutions, where regularity fixed $c_{2}$ as a function of $c_{1}$, the fluctuations should always be normalisable, such that the solutions behave as $y^{-2}$ towards the boundary. The remaining overall factor $c_{2}$ is undetermined because the equations of motion are linear. The requirement of regularity in the infrared can then only be satisfied by a discrete set of values for the meson mass $M$, which determines the spectrum. The result for the lowest lying meson modes is depicted in Figure 3.7.

With these results it is possible to return to the question of a holo- Goldstone graphic realisation of Goldstone's theorem. For the following discussion, it is important to keep in mind that the supergravity approximation in AdS/CFT correspondence implies being in the $N_{c} \rightarrow \infty$ limit, where the $\mathrm{U}(1)_{A}$ axial symmetry is non-anomalous in the field theory. A look at 
the large $N_{c}$ limit of QCD, where the $\eta^{\prime}$ becomes massless and thus a true Goldstone boson, inspires to look for the corresponding (pseudo-) Goldstone meson in this geometric setup.

A massless embedding with UV behaviour $z_{0}(y) \sim c y^{-2}$ restores the $\mathrm{U}(1)_{A} \simeq \mathrm{SO}(2)_{89}$ symmetry in the UV and therefore shows spontaneous symmetry breaking. In particular that means that the embedding solution $z_{0} \mathrm{e}^{i \theta_{0}}$, has an undefined angle $\theta_{0}$ at the boundary, which acquires an arbitrary value along the flow, picked out spontaneously by the dynamics. Clearly any fluctuation in the $\theta$ angle simply corresponds to a rotation into an-because of the presence of the U(1) - equivalent but different value of $\theta_{0}$. Since these values are all equivalent, the fluctuation in the $\theta$ direction should be a flat direction in the potential and correspond to a massless meson.

When the $\mathrm{U}(1)_{A}$ symmetry is explicitly broken in the UV by the quark mass $\left(z_{0} \sim m_{q}+c\left(m_{q}\right) y^{-2}\right)$, fluctuations in the angular direction do not rotate into an equivalent embedding and are therefore expected to become massive. Figure 3.7 shows that this holographic version of the Goldstone theorem is indeed realised. Furthermore beyond a certain quark mass, supersymmetry is restored and the meson masses become degenerate. For small quark mass, Figure 3.7(b), accordance with a prediction from effective field theory is found, the Gell-Mann-Oakes-Renner (GMOR) relation [84]

$$
M_{\pi}^{2}=\frac{m_{q}\langle\bar{\psi} \psi\rangle}{N_{f} f_{\pi}^{2}} .
$$

\subsection{Highly Excited Mesons}

In this Section inspired by a similar analysis in [39], the highly excited meson spectrum in the present background shall be investigated. In AdS/CFT this corresponds to considering mesons with large radial excitation number $n$. According to [40] the semi-classical approximation becoming valid in this limit gives rise to a restoration of chiral symmetry, because its breaking resulted from quantum effects at one-loop order which are suppressed for $S \gg \hbar$. 


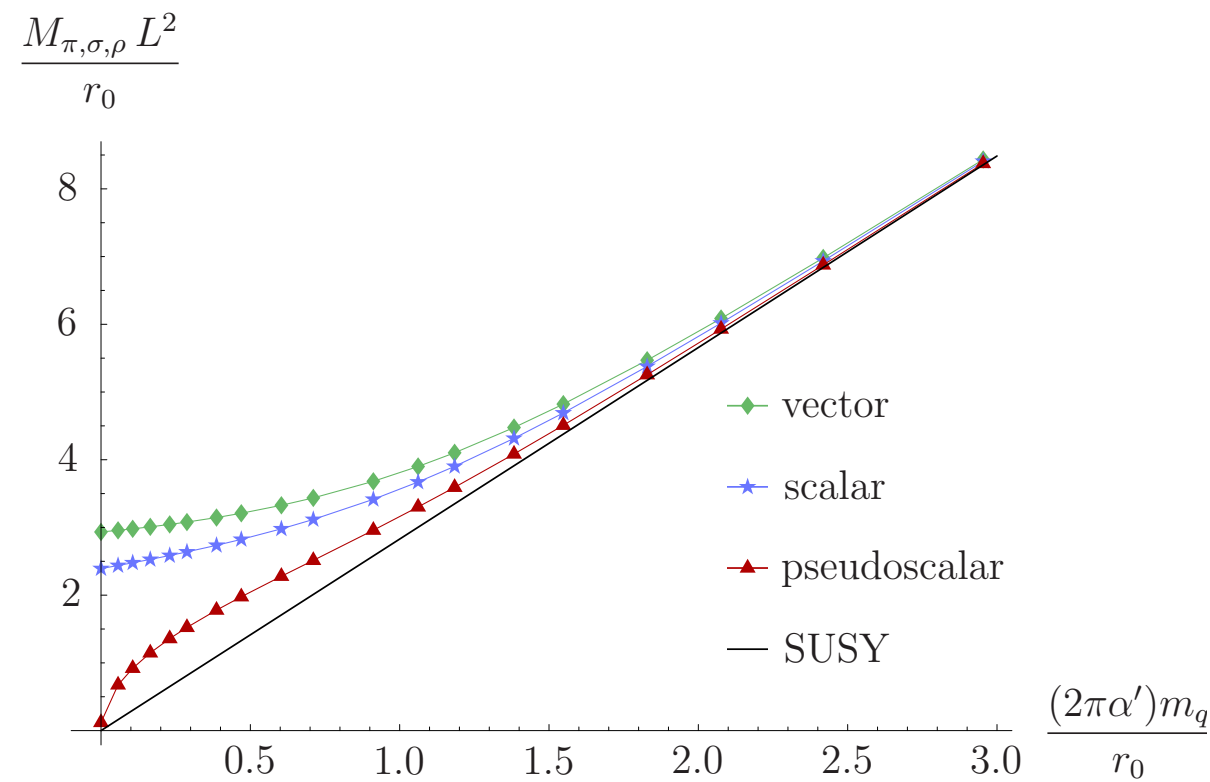

(a) Lightest mesons

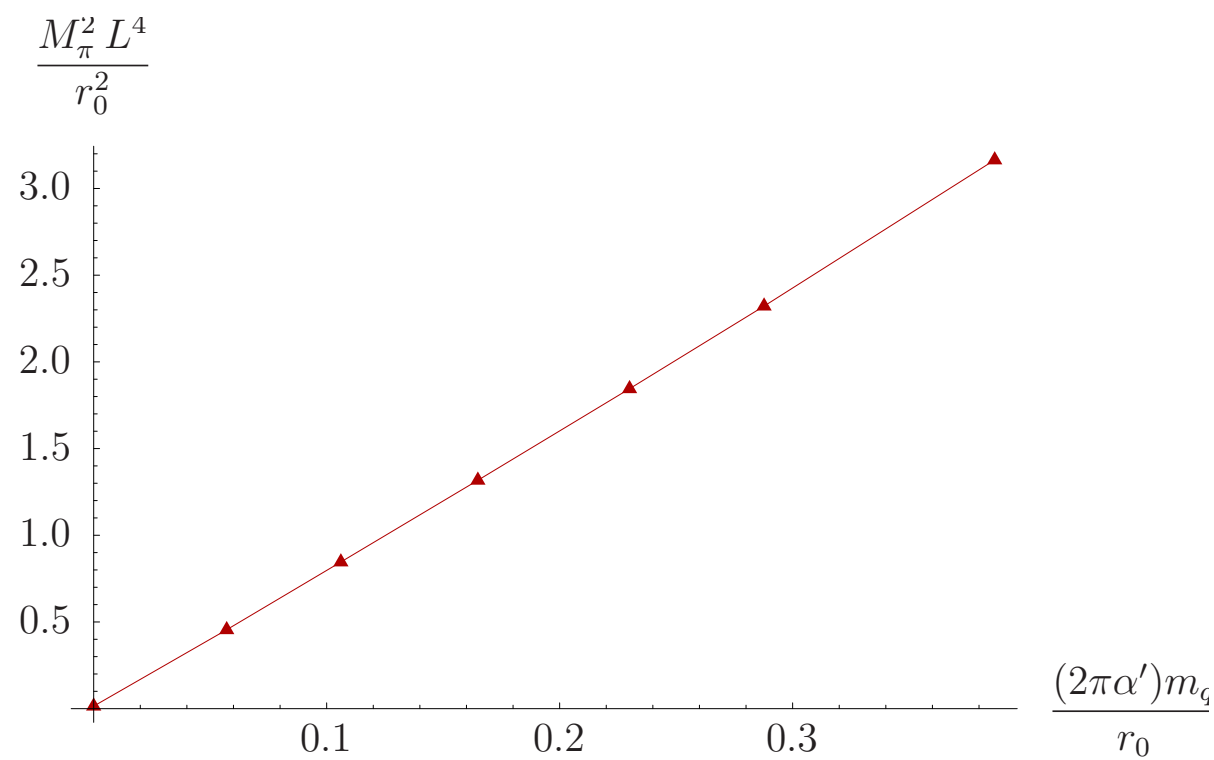

(b) Pseudoscalar meson and GMOR relation: $M_{\pi} \sim \sqrt{m_{q}}$

Figure 3.7: Plot (a) shows the lightest vector, scalar and pseudoscalar meson (in order of decreasing mass). While the scalar and vector meson retain a mass, the pseudoscalar meson becomes massless and therefore a true Goldstone boson in the limit $m_{q} \rightarrow 0$. Furthermore its mass exhibits a square root behaviour as predicted from effective field theory, plot (b). For large quark masses, supersymmetry is restored and the analytic SUSY result $M(n=0, \ell=0)=\frac{2 m_{q}}{L^{2}} \sqrt{2}$ is reproduced (black straight line). 


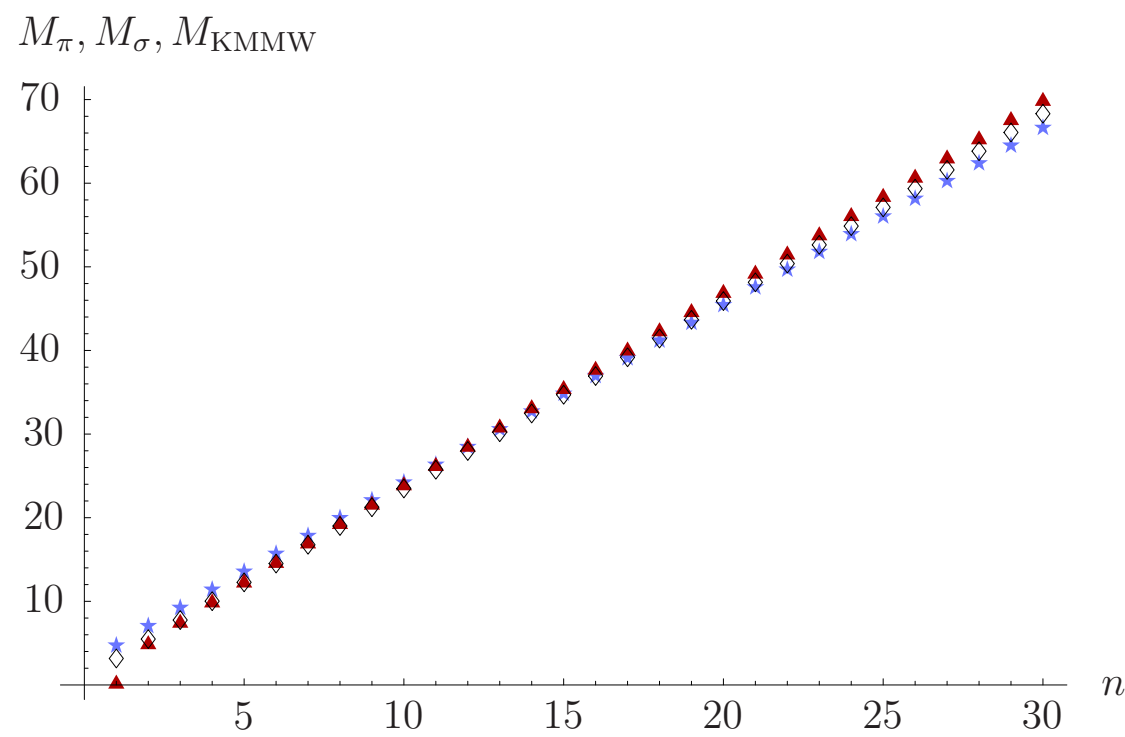

(a) Highly Excited Mesons

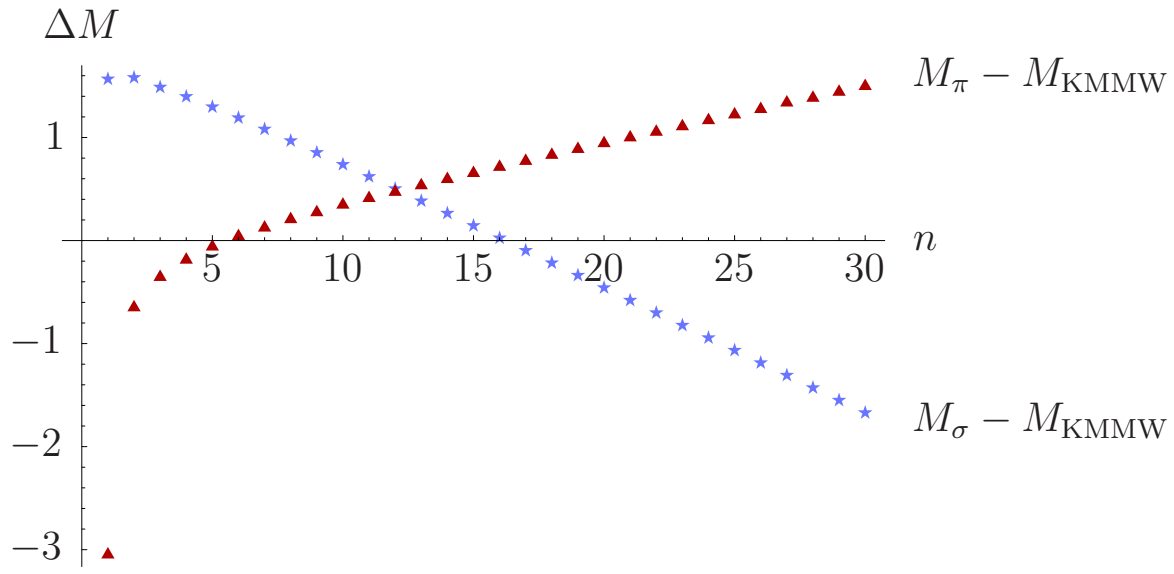

(b) Difference to SUSY Case

Figure 3.8: These plots show highly radially excited mesons for the $m_{q}=0$ embedding (with $r_{0}=L=1$ for numerics). For the analytically solvable SUSY case, this corresponds to $n \gg 1$ and therefore $M_{\mathrm{KMMW}}=2 \sqrt{(n+\ell+1)(n+\ell+2)} \sim 2 n$. While the proportionality to $n$ is preserved in the deformed case, the overall slope of the SUSY case is different and has been adjusted by multiplying $M_{\mathrm{KMMW}}$ by 1.15 for comparison. The difference to this rescaled mass as depicted in plot (b) suggests that the mass of the scalar and pseudoscalar mesons is not degenerate in the limit of large excitations. 
[39] found the rather generic behaviour

$$
M_{n} \sim n, \quad n \gg 1,
$$

for holographic duals of QCD-like theories. This is not in accordance with field theoretic expectations [41], which can be derived from simple scaling arguments: The length of the flux tube spanned between two ultra- scaling relativistic quarks of energy $E=p=M_{n} / 2$ is

$$
L \sim \frac{M_{n}}{\Lambda_{Q C D}^{2}}
$$

such that from the quasi-classical quantisation condition

$$
\int p d x \sim p L \sim \frac{M_{n}^{2}}{\Lambda_{Q C D}} \sim n,
$$

one reads off

$$
M_{n} \sim \sqrt{n}
$$

This is in contradiction to the results (3.30) and also the numerical results one obtains for the Gubser background shown in Figure 3.8. However this behaviour was to be expected since it is also found in the analytic spectrum of Kruczenski et al.

A to some extent related question is whether the difference $\delta M_{n}$ of chiral symmetry the scalar and pseudoscalar meson mass shows the right field theoretic restoration behaviour, which has been predicted to be $\left|\delta M_{n}\right| \lesssim n^{-3 / 2} \Lambda_{Q C D}$ with alternating sign for $\delta M_{n}[41]$.

While the analytic supersymmetric case fulfils this requirement trivially $\delta M_{n}=0$, interestingly this seems not to be the case for the Gubser background as can be seen in Figure 3.8. Actually $\delta M_{n}$ even could not be shown to vanish at all in the limit $n \rightarrow \infty$ implying that neither chiral symmetry nor supersymmetry is restored.

Having a closer look at the behaviour of such highly excited mesons, cf. Figure 3.9, one notices that the effect of large radial excitation is that the interior of the holographic space corresponding to the field theory's infrared is probed more densely. This suggests that for highly excited 
mesons in such a holographic description infrared effects might indeed not be sufficiently suppressed. On the other hand it seems surprising that mass degeneracy is not restored contrary to the case of large quark mass, where the mesons end up in the supersymmetric regime and do become degenerate as has been demonstrated in Figure 3.7.

Currently the method for calculating the meson spectrum inherently requires expansion to quadratic order in fluctuations. It would certainly be interesting to extend this procedure to include higher order contributions and reexamine the question of whether at least restoration of mass degeneracy can be achieved in the limit of highly radial excitation. 


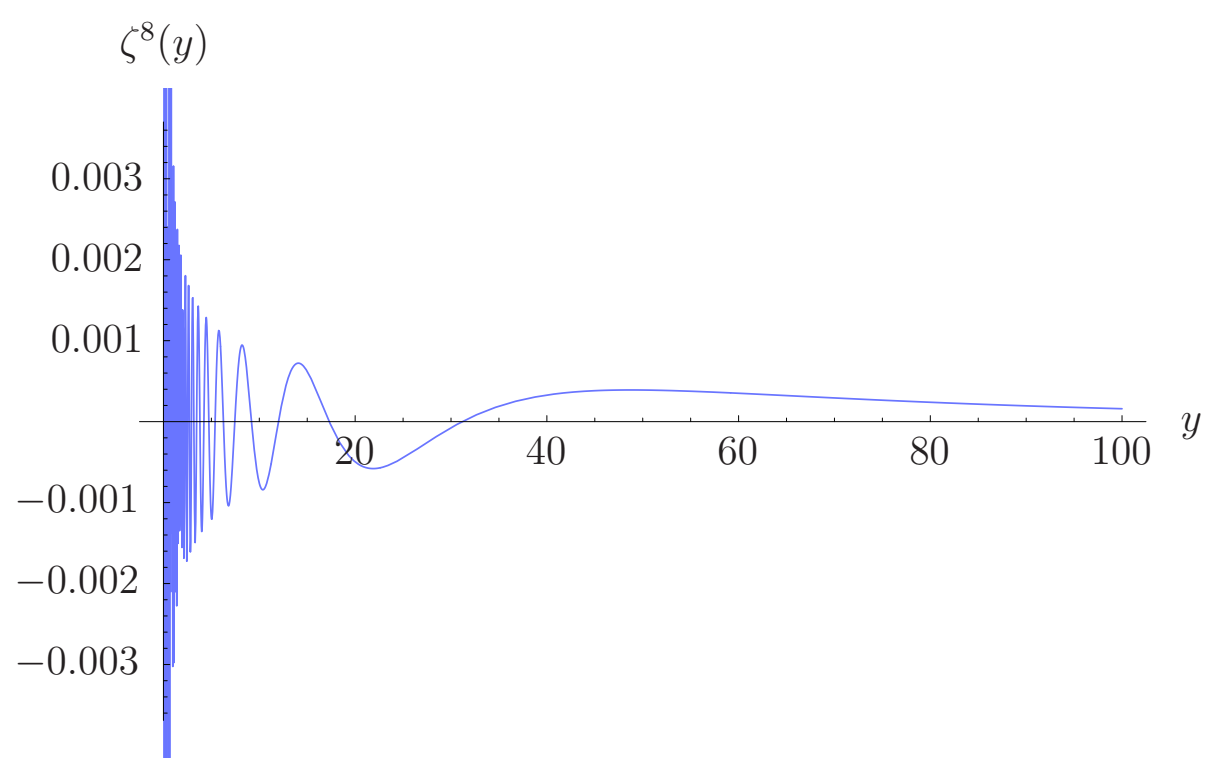

(a) Strong IR dependence

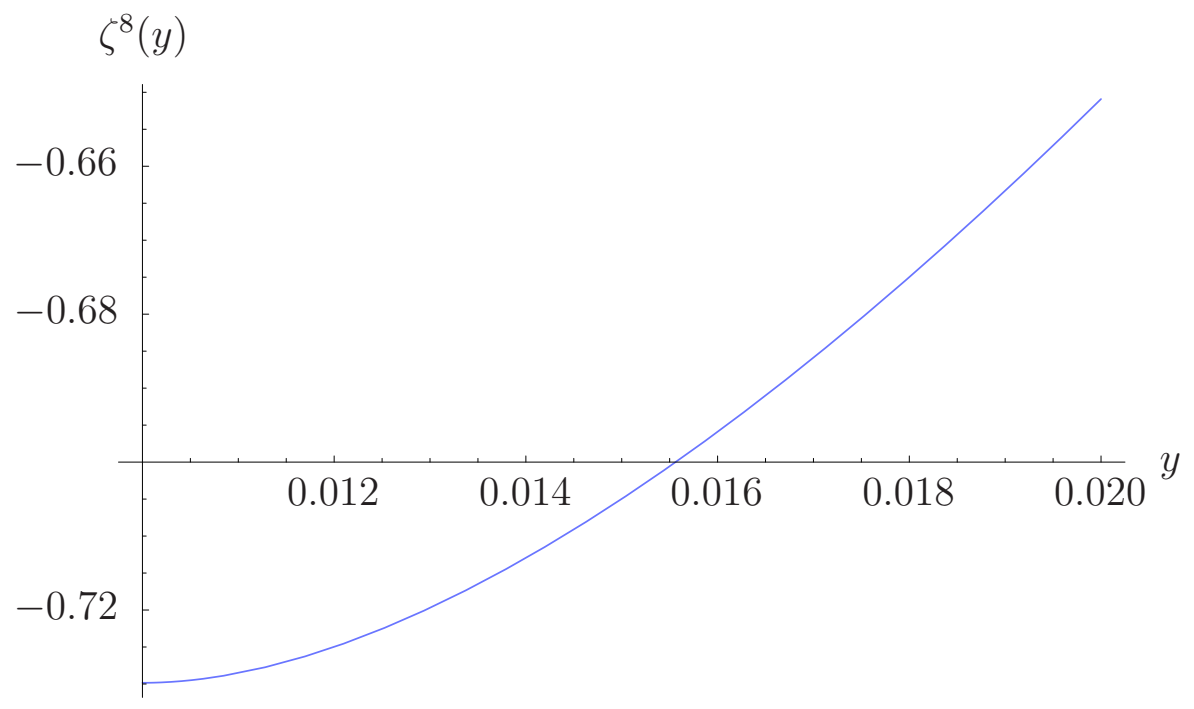

(b) IR Regularity

Figure 3.9: Pseudoscalar meson solution with excitation number $n=49$; i.e. the solution plotted in (a) has 49 zeros. Most of them concentrate in the far IR, but the solution is still smooth close to the centre (b). Increasing the excitation number scans the IR in more detail, where scalar and pseudoscalar meson mass are different. Therefore it is not expected to find mass degeneracy when increasing $n$ further. (Note that Cartesian fluctuations as opposed to polar fluctuations in $z$ and $\theta$ have been plotted. The mass spectrum is independent of this choice.) 

When I'm working on a problem, I never think about beauty. I think only how to solve the problem. But when I have finished, if the solution is not beautiful, I know it is wrong.

R. Buckminster Fuller

\section{Chapter 4}

\section{Second Deformation: Gauge Fields}

$\S 4.1$ Introduction, 55. $\S 4.2$ Conventions, 56. $\S 4.3$ Dual Field Theory, 57. $§ 4.3 .1$ Higgs Branch, 58. §4.4 Supergravity, 60. §4.4.1 Instantons, 60. §4.4.2 D7-brane Action, 62. $\S 4.5$ Meson Spectrum, 64. §4.5.1 Vector Fluctuations, 65. §4.5.2 Scalar Fluctuations, 71.

\subsection{Introduction}

In this Chapter the meson spectrum of the Higgs branch of the particular $\mathcal{N}=2$ super-Yang-Mills (SYM) theory (4.3) that can be described by a D3/D7-brane system [20] in the framework of AdS/CFT correspondence [79] will be determined. The analogous calculations for the Coulomb branch can be performed analytically [24], see Chapter 2, and can be made contact to in the cases of zero and infinite Higgs vacuum expectation value (VEV).

The work presented here is intrinsically a generalisation of the D3/D7 system of Chapter 2 to the case of more than one D7 brane, which corresponds to having multiple quark flavours. In particular, an additional effect that goes beyond simply having multiple copies of the Abelian case is considered. On the supergravity side it arises from the Wess-Zumino 
term in the D7-brane action, allowing four-dimensional instanton configurations to be classical solutions of the D7-brane gauge fields. On the field theory side this corresponds to switching on a vacuum expectation value (VEV) for the fundamental hypermultiplet. The field theory is therefore on the Higgs branch.

In the following Sections, the dual field theory will be presented and the exact notion of "Higgs branch" (which actually is a mixed CoulombHiggs branch) will be clarified. A short review of the BPST instanton solution is given.

The equations of motions are derived that determine the vector meson spectrum, which is calculated numerically and discussed analytically in the limits of small and large Higgs VEV. Finally the operator dictionary is explained and the fluctuations corresponding to scalar mesons are shown to fall into the same supermultiplets.

\subsection{Conventions}

The main difference between this Chapter and the preceding ones is the use of a non-Abelian D7-brane action to extend the analysis of the SUSY D3/D7 system to the sector of two flavours $\left(N_{f}=2\right)$. Therefore, the introduction of non-Abelian gauge covariant derivatives

$$
\begin{aligned}
& \mathcal{D}_{a}=\partial_{a}+g A_{a}, \\
& F_{a b}=\partial_{a} A_{b}-\partial_{b} A_{a}+g\left[A_{a}, A_{b}\right],
\end{aligned}
$$

can no longer be avoided and in addition to the index conventions of Table 4.1, a few notations have to be established.

The indices $M, N, \ldots$ will also be used as $\mathrm{SU}(2)$ generator indices, with the convention $\varepsilon_{456}=1$ and the Hermitean Pauli matrices

$$
\begin{aligned}
\left(T_{4}, T_{5}, T_{6}\right) & =\left(\left(\begin{array}{ll}
0 & 1 \\
1 & 0
\end{array}\right),\left(\begin{array}{cc}
0 & -i \\
i & 0
\end{array}\right),\left(\begin{array}{cc}
1 & 0 \\
-1 & 0
\end{array}\right)\right), \\
T_{M} T_{N} & =i \varepsilon_{M N K} T_{K}, \quad \operatorname{Tr} T_{M} T_{N}=2 \delta_{M N},
\end{aligned}
$$




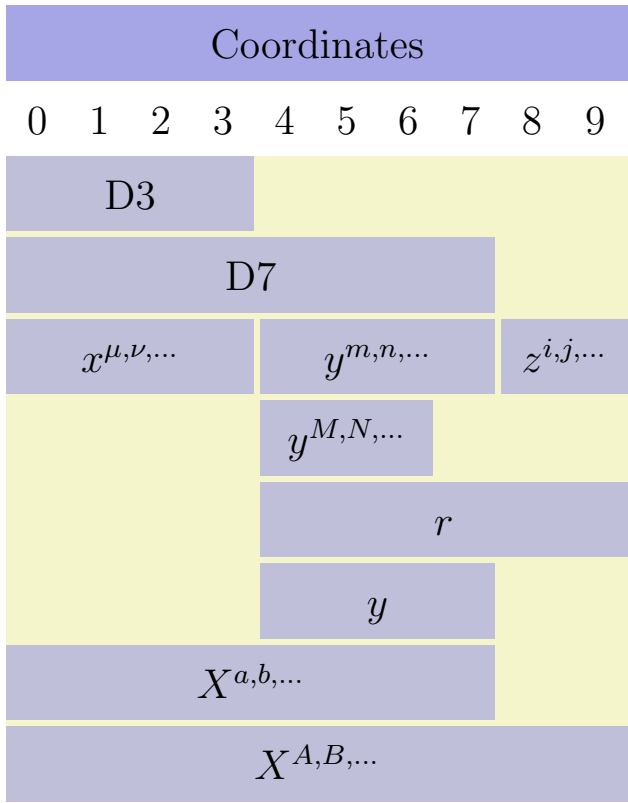

Table 4.1: Index Conventions

which allows to introduce the (anti-Hermitean) quaternion basis

quaternion basis

$$
\sigma_{4,5,6,7}=\left(i T_{4,5,6}, \mathbb{1}\right)
$$

The reader shall be reminded that in this basis $\mathrm{SO}(4)_{4567}$ transformations of $y^{m}$ can be also written as

$$
y^{m} \sigma_{m} \mapsto y^{m} U_{L} \sigma_{m} U_{R}
$$

with $U_{L}$ and $U_{R}$ elements of $\mathrm{SU}(2)_{L}$ and $\mathrm{SU}(2)_{R}$ respectively. Since the vector $\left(0,0,0, y^{7}\right)$ is invariant under transformations $U_{L}=\left(U_{R}\right)^{-1}$, rotations in the first three coordinates $\mathrm{SO}(3)_{456}$ can be identified with the diagonal subgroup $\operatorname{diag}\left[\mathrm{SU}(2)_{L} \times \mathrm{SU}(2)_{R}\right]$.

\subsection{Dual Field Theory}

On the string theory side, the setup discussed here is that of a stack of D3-branes and a parallel stack of D7s. In the decoupling limit, this amounts to considering type IIB supergravity (SUGRA) on $\mathrm{AdS}_{5} \times \mathrm{S}^{5}$ with $N_{f}$ probe D7-branes, which is dual to an $\mathcal{N}=2$ gauge theory obtained from coupling $N_{f} \mathcal{N}=2$ hypermultiplets in the fundamental representa- 
tion to $\mathcal{N}=4 \mathrm{SU}\left(N_{c}\right)$ SYM $[20]$.

In $\mathcal{N}=1$ language the Lagrangean of the dual field theory is

$$
\begin{aligned}
\mathscr{L}= & \int d^{4} \theta \operatorname{Tr}\left(\bar{\Phi}^{i} \mathrm{e}^{2 V} \Phi^{i} \mathrm{e}^{-2 V}+Q_{i}^{\dagger} \mathrm{e}^{2 V} Q^{i}+\tilde{Q}_{i} \mathrm{e}^{2 V} \tilde{Q}^{i \dagger}\right) \\
& +\left[\frac{1}{4 g^{2}} \int d^{2} \theta W_{\alpha} W^{\alpha}+\int d^{2} \theta W+\text { c.c. }\right]
\end{aligned}
$$

where the chiral fields $\Phi_{1,2,3}$ and the gauge field $V$ build up the $\mathcal{N}=4$ adjoint hypermultiplet, which in turn can be split into an $\mathcal{N}=2$ adjoint hypermultiplet composed of $\Phi_{1,2}$ and an $\mathcal{N}=2$ adjoint gauge multiplet of $V$ and $\Phi_{3}$. $Q^{i}$ and $\tilde{Q}_{i}$ are the $N_{f}$ chiral fields that build up the $\mathcal{N}=2$ fundamental hypermultiplet, and the superpotential is

$$
W=\operatorname{Tr}\left(\epsilon_{I J K} \Phi_{I} \Phi_{J} \Phi_{K}\right)+\tilde{Q}_{i}\left(m_{q}+\Phi_{3}\right) Q^{i}
$$

stability

At finite $N_{c}$ this theory is not asymptotically free, and the corresponding string background suffers from an uncancelled tadpole. However, in the strict probe limit $N_{f} / N_{c} \rightarrow 0$, the contributions to the 't Hooft couplings $\beta$ function, which scale like $N_{f} / N_{c}$, are suppressed. Furthermore the dual AdS string background has no tadpole problem because the probe D7-branes wrap a contractible $\mathrm{S}^{3}$. Although contractible, the background is stable, since the tachyon associated with shrinking the $\mathrm{S}^{3}$ satisfies (saturates) the Breitenlohner-Freedman bound [21]. Moreover the $\mathrm{AdS}_{5} \times \mathrm{S}^{3}$ embedding has been shown to be supersymmetric [85].

\subsubsection{Higgs Branch}

In terms of $\mathcal{N}=2$ multiplets, the theory consists of an adjoint gauge and hypermultiplet, which form the $\mathcal{N}=4$ hypermultiplet of $\mathcal{N}=4 \mathrm{SU}\left(N_{c}\right)$ SYM, and $N_{f}$ fundamental hypermultiplets. When the scalars of the latter acquire a VEV, the theory is on the Higgs branch.

While the scalars $\phi_{1,2}$ of the adjoint hypermultiplet independently may also have a VEV, VEVs of the $\mathcal{N}=2$ gauge multiplet's scalar $\phi_{3}$ prohibit a VEV for the fundamental hypermultiplets. Refining the discussion for the components gives rise to the mixed Coulomb-Higgs branch. The superpo- 
tential in $\mathcal{N}=1$ language is*

$$
W=\operatorname{Tr}\left(\epsilon_{I J K} \Phi_{I} \Phi_{J} \Phi_{K}\right)+\tilde{Q}_{i}\left(m_{q}+\Phi_{3}\right) Q^{i}
$$

with index $i$ enumerating the $N_{f}=2$ hypermultiplets.

Assume that a small number $k$ of the components of $\phi_{3}$ obtain a VEV,

$$
\left(\phi_{3}\right)_{N_{c} \times N_{c}}=\left(\begin{array}{cccccc}
0 & & & & & \\
& \ddots & & & & \\
& & 0 & & & \\
& & & -v & & \\
& & & \ddots & \\
& & & & & -v
\end{array}\right),
$$

which is dual to separating out $k$ D3-branes from the stack, and moreover that these VEVs are exactly such that some of the components of $m+\left\langle\phi_{3}\right\rangle$ vanish, $v=m$, which is dual to the singled out D3-branes coinciding with the D7-branes. Then F-flatness conditions $\tilde{q}_{i}\left(\phi_{3}+m\right)=\left(\phi_{3}+m\right) q^{i}=0$ permit the corresponding $2 k$ components of the fundamental hypermultiplet to also acquire a non-vanishing VEV

$$
\left(q^{i}\right)_{N_{c} \times 1}=\left(\begin{array}{c}
0 \\
\vdots \\
0 \\
\alpha_{1}^{i} \\
\vdots \\
\alpha_{k}^{i}
\end{array}\right), \quad\left(\tilde{q}_{i}\right)_{1 \times N_{c}}=\left(\begin{array}{llllll}
0 & \cdots & 0 & \beta_{1 i} & \cdots & \beta_{k i}
\end{array}\right) \text {. }
$$

These VEVs, which are further constrained by additional F- and D-flatness conditions, are the string theory dual of the D3-branes that coincide with the D7-branes to be dissolved [86] in the D7-branes and form instantons dissolved branes in the gauge fields of the D7s. This process is caused by the Wess-Zumino coupling $S_{W Z} \sim \int P\left[C_{(4)}\right] \wedge F \wedge F$. Note that the backreaction of the

${ }^{*}$ There are three adjoint $\mathcal{N}=1$ chiral fields $\Phi_{1,2,3}$ with lowest components $\phi_{1,2,3}$ and one real field $V$, which forms an $\mathcal{N}=2$ gauge multiplet with $\Phi_{3}$. The $N_{f}$ chiral fields $Q^{i}$ and $\tilde{Q}_{i}$ make up the $\mathcal{N}=2$ fundamental hypermultiplet and have lowest components $q^{i}$ and $\tilde{q}_{i}$. 
dissolved D3-branes can only be neglected when their number $k$ is small in comparison to $N_{c}$. Specifically in this Chapter $k=1$ will be assumed.

Taking into account the breaking of $\mathrm{SU}(2)_{R} \times \mathrm{SU}(2)_{f}$ to its diagonal subgroup, which is mediated by the instanton configuration on the supergravity side, the structure of the VEVs is as follows

$$
\left(\phi_{3}\right)_{N_{c} \times N_{c}}=\left(\begin{array}{cccc}
0 & & & \\
& \ddots & & \\
& & 0 & \\
& & & -m
\end{array}\right), \quad\left(\mathfrak{q}_{\alpha}^{i}\right)=\left(\begin{array}{c}
0 \\
\vdots \\
0 \\
\varepsilon_{i \alpha} \Lambda
\end{array}\right),
$$

with $\alpha=1,2$ the $\mathrm{SU}(2)_{R}$ index and $\mathfrak{q}_{1}=q, \mathfrak{q}_{2}=\tilde{q}$.

\subsection{Supergravity}

\subsubsection{Instantons}

In Yang-Mills (YM) theories, instantons arise as finite action solutions from the semi-classical approximation to path integrals, which requires to find all solutions that minimise the Euclidian action. These solutions, (anti-)self dual gauge field configuration of arbitrary topological charge $k$, can be found from a set of algebraic equations, the so-called ADHM constraints due to Atiyah, Drinfeld, Hitchin and Manin. These equations are non-linear and cannot be solved in general because of their complex structure, though there has been recent progress in AdS/CFT inspired large $N_{c}$ considerations [87]. In particular the four dimensional ADHM constraints arise from $\mathrm{D}$ and F-flatness conditions of $\mathrm{D}(p+4)$-branes probed by $\mathrm{D} p$-branes $[88,89]$.

Although the ADHM formalism works for all non-exceptional groups, the focus here will be on $\mathrm{SU}(N)$ theories in Euclidian space. Consider the following action,

$$
S=-\frac{1}{2} \int d^{4} x \operatorname{Tr} F_{m n}^{2}+i \theta k
$$


with the topological charge and field strength

$$
\begin{aligned}
k & :=-\frac{g^{2}}{16 \pi^{2}} \int d^{4} y \operatorname{Tr} F_{m n}{ }^{*} F_{m n}, \quad k \in \mathbb{Z}, \\
F_{m n} & :=\partial_{m} A_{n}-\partial_{n} A_{m}+g\left[A_{m}, A_{n}\right], \\
{ }^{*} F_{m n} & :=\frac{1}{2} \varepsilon_{m n k l} F_{k l}
\end{aligned}
$$

and anti-Hermitean gauge field $A_{m}$ such that the covariant derivative reads $\mathcal{D}_{m}=\partial_{m}+g A_{m}$.

Instantons with negative topological charge, also known as anti-instantons, will not be considered here. The action is minimised by self dual solutions

$$
\begin{aligned}
{ }^{*} F_{m n} & = \pm F_{m n}, \\
\Longrightarrow S & =-2 \pi i k \tau \quad k>0,
\end{aligned}
$$

with the complex coupling $\tau=\frac{4 \pi i}{g^{2}}+\frac{\theta}{2 \pi}$.

The self-dual SU(2) instanton solution, also known as the BelavinPolyakov-Shvarts-Tyupkin (BPST) instanton [90], is given by

$$
A_{n}^{\mathrm{inst}}=g^{-1} \frac{2\left(y_{m}-Y_{m}\right) \sigma_{m n}}{(y-Y)^{2}+\Lambda^{2}}, \quad F_{m n}^{\mathrm{inst}}=g^{-1} \frac{4 \rho^{2} \sigma_{m n}}{\left((y-Y)^{2}+\Lambda^{2}\right)^{2}},
$$

with the instanton moduli $\Lambda$ (size) and $Y^{m}$ (position). The Lorentz generators are given by

$$
\sigma_{m n}=\frac{1}{4}\left(\sigma_{m} \bar{\sigma}_{n}-\sigma_{n} \bar{\sigma}_{m}\right), \quad \bar{\sigma}_{m n}=\frac{1}{4}\left(\bar{\sigma}_{m} \sigma_{n}-\bar{\sigma}_{n} \sigma_{m}\right)
$$

and it holds

$$
\sigma_{m n}=\frac{1}{2} \varepsilon_{m n k l} \sigma_{k l}, \quad \quad \bar{\sigma}_{m n}=-\frac{1}{2} \varepsilon_{m n k l} \bar{\sigma}_{k l} .
$$

The above identification of gauge indices with vector indices expresses the instanton breaking the $\mathrm{SU}(2)_{L} \times \mathrm{SU}(2)$ to its diagonal subgroup, with $\mathrm{SU}(2)_{L}$ from the double covering group of the Euclidian Lorentz group $\mathrm{SO}(4)$ and $\mathrm{SU}(2)$ the gauge group.

The BPST instanton falls off slowly for large distances, which creates 
convergence problems of various integrals. A well known solution in the instanton literature is the use of a singular gauge transformation

$$
U(y):=\frac{\sigma_{m}(y-Y)^{m}}{|y-Y|}
$$

which transforms the non-singular instanton solution to a singular one,

$$
A_{n}=g^{-1} \frac{2 \Lambda^{2}(y-Y)_{m} \bar{\sigma}_{m n}}{(y-Y)^{2}\left[(y-Y)^{2}+\Lambda^{2}\right]}
$$

that has better large distance behaviour. This particular gauge transformation also associates $\mathrm{SU}(2)_{R}$ with the gauge group, such that (4.17) breaks the $\mathrm{SU}(2)_{L} \times \mathrm{SU}(2)_{R} \times \mathrm{SU}(2)$ to $\mathrm{SU}(2)_{L} \times \operatorname{diag}\left[\mathrm{SU}(2)_{R} \times \mathrm{SU}(2)\right]$. Note that also in the instanton literature a known consequence of (4.16) is the modification of boundary terms. Therefore consequences for the AdS/CFT dictionary are also to be expected.

\subsubsection{D7-brane Action}

As a reminder the $A d S_{5} \times \mathrm{S}^{5}$ background as given in (2.1), (2.5) is

$$
d s^{2}=H^{-1 / 2}(r) \eta_{\mu \nu} d x^{\mu} d x^{\nu}+H^{1 / 2}(r)\left(d \vec{y}^{2}+d \vec{z}^{2}\right),
$$

with

$$
\begin{aligned}
H(r) & =\frac{L^{4}}{r^{4}}, & r^{2} & =\vec{y}^{2}+\vec{z}^{2}, \\
L^{4} & =4 \pi g_{s} N_{c}\left(\alpha^{\prime}\right)^{2}, & \vec{y}^{2} & =\sum_{m=4}^{7} y^{m} y^{m}, \\
C_{0123}^{(4)} & =H^{-1}, & \vec{z}^{2} & =\left(z^{8}\right)^{2}+\left(z^{9}\right)^{2}, \\
\mathrm{e}^{\varphi} & =\mathrm{e}^{\varphi_{\infty}}=g_{s} . & &
\end{aligned}
$$

The constant embedding

$$
z^{8}=0, \quad \quad z^{9}=\tilde{m}_{q}
$$


defines the distance $\tilde{m}_{q}=\left(2 \pi \alpha^{\prime}\right) m_{q}$ between the D3 and D7-branes and therefore determines the mass $m_{q}$ of the fundamental hypermultiplet.

Moreover it yields the induced metric (2.3)

$$
\begin{gathered}
d s_{\mathrm{D} 7}^{2}=H^{-1 / 2}(r) \eta_{\mu \nu} d x^{\mu} d x^{\nu}+H^{1 / 2}(r) d \vec{y}^{2}, \\
r^{2}=y^{2}+\left(2 \pi \alpha^{\prime}\right)^{2} m_{q}^{2}, \quad y^{2} \equiv y^{m} y^{m}
\end{gathered}
$$

on the D7-brane.

At quadratic order, the non-Abelian DBI action (1.31) and the WessZumino term (1.25) are respectively

$$
\begin{aligned}
& S_{\mathrm{DBI}}=-\mu_{7} \int d^{p+1} \xi \mathrm{STr}^{-\varphi} \sqrt{-\operatorname{det} G_{a b}}\left[\frac{\lambda^{2}}{2} \mathcal{D}_{a} \Phi_{i} \mathcal{D}^{a} \Phi^{i}+\frac{\lambda^{2}}{4} F_{a b} F^{a b}\right] \\
& =-\frac{T_{7} \gamma^{2}}{4} \int d^{4} x d^{4} y \operatorname{Tr}\left[-2 H(r) \mathcal{D}_{\mu} \Phi \mathcal{D}_{\mu} \bar{\Phi}+2 \mathcal{D}_{m} \Phi \mathcal{D}_{m} \bar{\Phi}+\right. \\
& H(r) F_{\mu \nu} F_{\mu \nu}+2 F_{m \nu} F_{m \nu}+ \\
& \left.H^{-1}(r) F_{m n} F_{m n}\right] \text {, } \\
& S_{W Z}=T_{7} \int \operatorname{STr} \frac{\gamma^{2}}{2} P\left[C^{(4)}\right] \wedge F \wedge F \\
& =T_{7} \frac{\gamma^{2}}{4} \int \operatorname{Tr} H^{-1}(r) F_{m n} \frac{1}{2} F_{r s} d x^{0} \wedge \ldots d x^{3} \wedge \underbrace{d y^{m} \wedge d y^{n} \wedge d y^{r} \wedge d y^{s}}_{=\varepsilon_{m n r s} d y^{4} \wedge d y^{5} \wedge d y^{6} \wedge d y^{7}} \\
& =T_{7} \frac{\gamma^{2}}{4} \int d^{4} x d^{4} y H^{-1}(r) \operatorname{Tr} F_{m n}{ }^{*} F_{m n},
\end{aligned}
$$

where $\Phi, \bar{\Phi}=\Phi^{9} \pm i \Phi^{8}, \gamma=2 \pi \alpha^{\prime}$ and the Hodge dual is ${ }^{*} F_{m n}:=\frac{1}{2} \varepsilon_{m n r s} F_{r s}$, with the epsilon symbol $\varepsilon_{4567}=1$. All indices have been lowered and are now contracted with a Minkowski metric $\eta_{a b}=\left(\eta_{\mu \nu}, \delta_{m n}\right)$. This will be true for all subsequent expressions in this Chapter, providing a convenient framework for the discussion of solutions that are self-dual with respect to the flat metric $\delta_{m n}$.

These solutions arise because there is a (known, cf. [88, 89]) correspon- DBI/WZ dence between instantons and the Higgs branch. The discussion in this the- conspiracy sis will be confined to quadratic order, ${ }^{*}$ where the DBI and Wess-Zumino

${ }^{*}$ The explicit expanded form of the non-Abelian DBI action is only known to finite 
term due to $F_{m n}\left(F_{m n}-{ }^{*} F_{m n}\right)=2 F_{m n}^{-} F_{m n}^{-}$complement one another to yield

$$
\begin{gathered}
S=-\frac{T_{7} \gamma^{2}}{4} \int d^{4} x d^{4} y \operatorname{Tr}\left[-2 H(r) \mathcal{D}_{\mu} \Phi \mathcal{D}_{\mu} \bar{\Phi}+2 \mathcal{D}_{m} \Phi \mathcal{D}_{m} \bar{\Phi}+\right. \\
H(r) F_{\mu \nu} F_{\mu \nu}+2 F_{m \nu} F_{m \nu}+ \\
\left.2 H^{-1}(r) F_{m n}^{-} F_{m n}^{-}\right]
\end{gathered}
$$

This action is extremised by the configuration

$$
\begin{gathered}
F_{m n}^{-}=0, \\
\Phi=\tilde{m}_{q}, \quad F_{\mu \nu}=F_{m n}=0,
\end{gathered}
$$

which is manifestly self-dual with respect to the D3-transversal flat metric $\delta_{m n}$. The particular background configuration that will be investigated here,

$$
A_{m}=\frac{2 \Lambda^{2} \bar{\sigma}_{m n} y_{n}}{y^{2}\left(y^{2}+\Lambda^{2}\right)}, \quad A_{\mu}=0, \quad \Phi_{0}=\tilde{m}_{q}
$$

takes the singular gauge instanton (4.17) as an ansatz for (4.28) that brings the correct boundary behaviour for the AdS/CFT dictionary as will be seen below.

\subsection{Meson Spectrum}

Now the meson spectrum for fluctuations about the above background shall be calculated. Obviously there should be massless mesons corresponding to changes of the instanton moduli, size $(\Lambda)$ and position (not explicit in the above ansatz, since the instanton is simply located at $y_{m}=0$ ). These will be ignored and concentration will be instead on the more interesting fluctuations of the gauge fields and scalars. The simplest modes are vector fluctuations of type II, cf. eq. (2.22b), and scalar fluctuations, both in the same supermultiplet and in the the lowest representation of $\mathrm{SU}(2)_{L} \times \operatorname{diag}\left[\mathrm{SU}(2)_{R} \times \mathrm{SU}(2)_{f}\right]$. In particular this means that the fluc-

order, cf. [91] for terms at sixth order. The existence of instanton solutions puts constraints on unknown higher order terms [92, 93]. 
tuations will be assumed to be independent of angular variables in the D3-transversal/D7-longitudinal coordinates; i.e. in the language of the analytically solvable scenario of Chapter $2: \ell=0$.

\subsubsection{Vector Fluctuations}

In accordance with the coordinate splitting $X_{a}=x_{\mu}, y_{m}$ performed in the action (4.27), fluctuations of the form $\mathcal{A}:=A-A^{\text {inst }}$ will be considered. The simplest ansatz for gauge fluctuation, which at the same time is most interesting due to describing vector mesons, is given by "Type II" fluctuation (2.22b) in the language of Kruczenski et al., see Chapter 2. This particular ansatz is non-trivial in the D3-longitudinal components only, such that the simplest non-Abelian choice is a singlet under $\mathrm{SU}(2)_{L}$ and a triplet under $\operatorname{diag}\left[\mathrm{SU}(2)_{R} \times \mathrm{SU}(2)_{f}\right]$. An obvious ansatz is given by

$$
\mathcal{A}_{\mu}^{(a)}=\xi_{\mu}(k) f(y) \mathrm{e}^{i k_{\mu} x_{\mu}} T^{a}, \quad y^{2} \equiv y^{m} y^{m},
$$

and

$$
A_{\mu}=\mathcal{A}_{\mu}, \quad A_{m}=A_{m}^{\text {inst }}
$$

The Euler-Lagrange equations

$$
\begin{gathered}
\partial_{\mu} \frac{\partial \mathscr{L}}{\partial \partial_{\mu} A_{\nu}^{M}}+\partial_{m} \frac{\partial \mathscr{L}}{\partial \partial_{m} A_{\nu}^{M}}-\frac{\partial \mathscr{L}}{\partial A_{\nu}^{M}}=0 \\
\partial_{\mu} \frac{\partial \mathscr{L}}{\partial \partial_{\mu} A_{n}^{M}}+\partial_{m} \frac{\partial \mathscr{L}}{\partial \partial_{m} A_{n}^{M}}-\frac{\partial \mathscr{L}}{\partial A_{n}^{M}}=0
\end{gathered}
$$

for the action (4.27) are

$$
\begin{aligned}
\mathcal{D}_{\mu}\left(H F_{\mu \nu}\right)+\mathcal{D}_{m} F_{m \nu} & =0, \\
\mathcal{D}_{\mu} F_{\mu n}+2 \mathcal{D}_{m}\left[H^{-1} F_{m n}^{-}\right] & =0 .
\end{aligned}
$$

To linear order the former becomes $\partial_{\mu} \mathcal{A}_{\mu}=0$, which is solved by $k_{\mu} \xi_{\mu}=0$, while the latter reads

$$
\begin{aligned}
& H \partial_{\mu} \partial_{\mu} \mathcal{A}_{\nu}+\partial_{m} \partial_{m} \mathcal{A}_{\nu}+g \partial_{m}\left[A_{m}^{\text {inst }}, \mathcal{A}_{\nu}\right] \\
& \quad+g\left[A_{m}^{\text {inst }}, \partial_{m} \mathcal{A}_{\nu}\right]+g^{2}\left[A_{m}^{\text {inst }},\left[A_{m}^{\text {inst }}, \mathcal{A}_{\nu}\right]\right]=0,
\end{aligned}
$$


which for the ansatz (4.30) yields

$$
0=\left[\frac{M^{2} L^{4}}{\left(y^{2}+\left(2 \pi \alpha^{\prime}\right)^{2} m_{q}^{2}\right)^{2}}-\frac{8 \Lambda^{4}}{y^{2}\left(y^{2}+\Lambda^{2}\right)^{2}}+\frac{1}{y^{3}} \partial_{y}\left(y^{3} \partial_{y}\right)\right] f(y),
$$

where $M^{2}=-k_{\mu} k_{\mu}$ in accordance with having chosen a Minkowski metric with mostly plus convention for contraction of flat indices.

For numerics it is convenient to join the two parameters quark mass and instanton size by rescaling according to

$$
\tilde{y} \equiv \frac{y}{2 \pi \alpha^{\prime} m_{q}}, \quad \tilde{\Lambda} \equiv \frac{\Lambda}{2 \pi \alpha^{\prime} m_{q}}, \quad \tilde{M}^{2} \equiv \frac{M^{2} L^{4}}{\left(2 \pi \alpha^{\prime} m_{q}\right)^{2}},
$$

such that equation (4.37) becomes

$$
0=\left[\frac{\tilde{M}^{2}}{\left(\tilde{y}^{2}+1\right)^{2}}-\frac{8 \tilde{\Lambda}^{4}}{\tilde{y}^{2}\left(\tilde{y}^{2}+\tilde{\Lambda}^{2}\right)^{2}}+\frac{1}{\tilde{y}^{3}} \partial_{\tilde{y}}\left(\tilde{y}^{3} \partial_{\tilde{y}}\right)\right] f(\tilde{y})
$$

operator map

At large $\tilde{y}$ (4.39) has two linear independent solutions whose asymptotics are given by $\tilde{y}^{-w}$ with $w=0,2$. The normalisable solutions corresponding to vector meson states behave as $\tilde{y}^{-2}$ asymptotically. From standard AdS/CFT correspondence, one expects $w=\Delta$ and $w=4-\Delta$, where $\Delta$ is the UV conformal dimension of the lowest dimension operator with the quantum numbers of the vector meson. However, the kinetic term does not have a standard normalisation; i.e. the radial component of the Laplace operator appearing in the equation above is not (only) $\partial_{\tilde{y}}^{2}$, and consequently an extra factor of $\tilde{y}^{\alpha}$, for some $\alpha$, appears in the expected behaviour; so the exponents actually read $w=\alpha+\Delta, \alpha+4-\Delta$. From the difference it is read off that $\Delta=3$. The dimensions and quantum numbers are those of the $\mathrm{SU}(2)_{f}$ flavour current,

$$
\mathcal{J}_{\mu}^{b}=-\bar{\psi}^{ \pm i} \gamma_{\mu} \sigma^{b}{ }_{i j} \psi_{\mp}{ }^{j}+\bar{q}^{\alpha i} \stackrel{\leftrightarrow}{D}_{\mu} \sigma^{b}{ }_{i j} q_{\alpha}{ }^{j}
$$

with $\alpha$ the $\mathrm{SU}(2)_{R}$ index and $i, j$ the flavour indices. This current has $\mathrm{SU}(2)_{R} \times \mathrm{SU}(2)_{L} \times \mathrm{U}(1)$ quantum numbers $(0,0)_{0}$. 


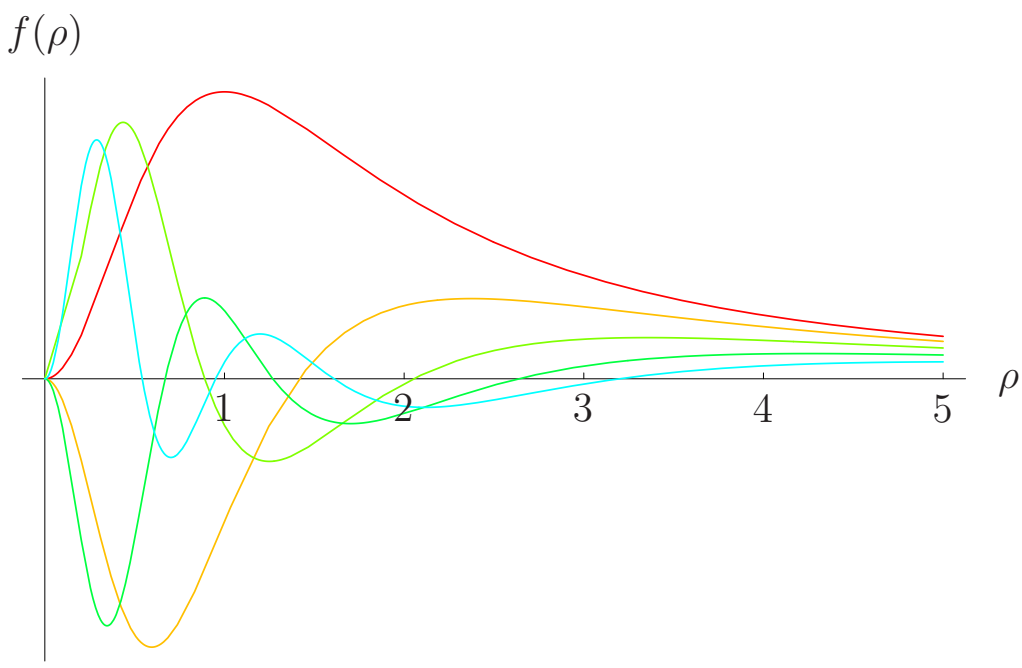

(a) Regular solutions of (4.39) in arbitrary units for $\Lambda=2 \pi \alpha^{\prime} m$

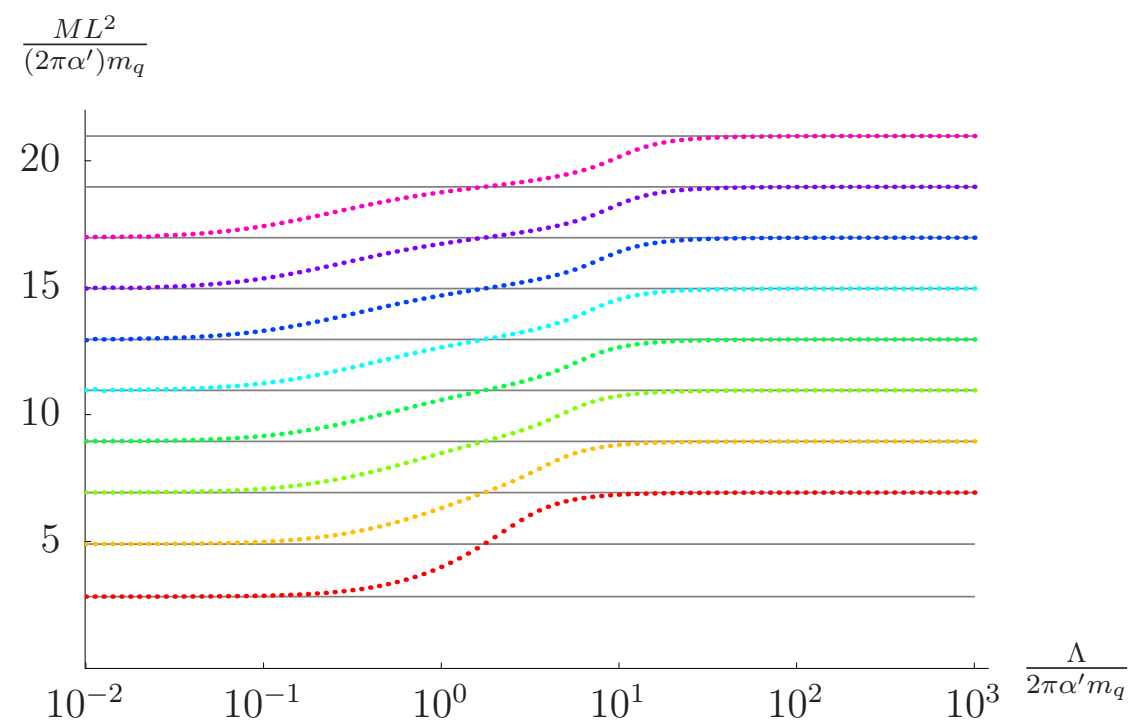

(b) Numerically determined meson masses.

Figure 4.1: Each dotted line represents a regular solution of the equation of motion, corresponding to a vector multiplet of mesons. Plot (a) shows the five regular solutions of (4.39) corresponding to the lightest meson masses in (b). The units on axis of ordinate in (a) are arbitrary because (4.39) is a linear equation. The vertical axis in (b) is $\sqrt{\lambda} M / m_{q}$ where $M$ is the meson mass, $\lambda$ the 't Hooft coupling and $m_{q}$ the quark mass. The horizontal axis is $v / m_{q}$, where $v=\Lambda / 2 \pi \alpha^{\prime}$ is the Higgs VEV. In the limits of zero and infinite instanton size (Higgs VEV), the spectrum (grey horizontal lines) obtained analytically in Chapter 2 is recovered. 
The asymptotic behaviour of the supergravity solution is

$$
\mathcal{A}_{b(a)}^{\mu}=\xi^{\mu}(k) \mathrm{e}^{i k \cdot x} f(\tilde{y}) \delta_{a b} \sim \tilde{y}^{-2}\left\langle a, \xi, k\left|\mathcal{J}_{b}^{\mu}(x)\right| 0\right\rangle,
$$

where $\mathcal{J}^{\mu}$ is the $\mathrm{SU}(2)_{f}$ flavour current and $|a, \xi, k\rangle$ is a vector meson with polarisation $\xi$, momentum $k$, and flavour triplet label $a$. Note that the index $b$ in $\mathcal{A}_{b(a)}^{\mu}$ is a Lie algebra index, whereas the index $(a)$ labels the flavour triplet of solutions.

meson spectrum The meson spectrum is numerically determined by a shooting technique using interval bisection to find the values $\tilde{M}^{2}$ that admit solutions to (4.39) that are regular $\left(c_{2}=0\right.$ for IR behaviour $\left.c_{1} \tilde{y}^{2}+c_{2} \tilde{y}^{-4}\right)$ and normalisable $\left(c_{1}=0\right.$ for UV behaviour $\left.c_{1}+c_{2} \tilde{y}^{-2}\right)$. The result for the lowest lying modes is shown in Figure 4.1.

why singular gauge

In passing it is noted that the second term in (4.39), which comes from the $g^{2}$ term in the equation of motion (4.36), is roughly the instanton squared and up to numerical constants would have been $y^{2} /\left(y^{2}+\Lambda^{2}\right)^{2}$ for the instanton in non-singular gauge. This contribution would have changed the UV behaviour of $f(y)$ and therefore prohibited to make contact to the SUSY case in the limit of zero instanton size, where (4.36) can be solved analytically.

asymptotics In the limit of infinite instanton size, one might expect the same spectrum since the field strength vanishes locally. This corresponds to infinite Higgs VEV in the field theory, which reduces the gauge group from $\mathrm{SU}\left(N_{c}\right)$ to $\mathrm{SU}\left(N_{c}-1\right)$. This difference is negligible in the large $N_{c}$ limit and one might expect to return to the origin of moduli space. However there is a non-trivial shift of the spectrum, which makes the flow from zero to infinite Higgs VEV not quite a closed loop as can be seen in Figure 4.1(b).

Since at both ends the analytic spectrum in reproduced, it should be possible to capture this behaviour in the equation of motion (4.36). Indeed a simultaneous treatment of both cases can be achieved by rewriting (4.36) in the suggestive form

$$
0=\left[\frac{\tilde{M}^{2}}{\left(\tilde{y}^{2}+1\right)^{2}}-\frac{\ell(\ell+2)}{\tilde{y}^{2}}+\frac{1}{\tilde{y}^{3}} \partial_{\tilde{y}}\left(\tilde{y}^{3} \partial_{\tilde{y}}\right)\right] f(\tilde{y}),
$$

with $\ell=0,2$ for zero or infinite $\tilde{\Lambda}$ respectively. 
This is the same equation (2.10) that was found for fluctuations about the trivial background $A^{a}=0$, but $\ell$ was given rise to by excitations on the internal manifold. The ansatz was

$$
\mathcal{A}^{\mu}=\xi^{\mu}(k) \mathrm{e}^{i k_{\mu} x^{\mu}} f(y) \mathcal{Y}^{\ell}\left(\mathrm{S}^{3}\right),
$$

with $\mathcal{Y}^{\ell}\left(\mathrm{S}^{3}\right)$ the scalar spherical harmonics on $\mathrm{S}^{3}$ transforming under $\left(\frac{\ell}{2}, \frac{\ell}{2}\right)$ representations of $\mathrm{SU}(2)_{L} \times \mathrm{SU}(2)_{R}$. [24] found that (4.42) can be solved analytically in terms of a hypergeometric function (2.12) parametrised by $n$ and $\ell$, which by regularity and normalisability become quantised and yield the discrete spectrum

$$
\tilde{M}^{2}=4(n+\ell+1)(n+\ell+2), \quad n, \ell \geq 0 .
$$

For intermediate values of the instanton size, a flow connecting the analytically known spectra is expected and could be confirmed numerically, see Figure 4.1(b).

It remains to comment on how it is possible to continuously transform a spherical harmonic in the $(0,0)$ of the unbroken $\mathrm{SU}(2)_{L} \times \mathrm{SU}(2)_{R}$ into singular gauge revisited one that transforms under the $(1,1)$, while $\mathrm{SU}(2)_{L}$ is unbroken along the flow.* The solution to this puzzle is that the instanton in singular gauge does not vanish in the limit of large instanton size, while in non-singular gauge it does. So the spectrum at large instanton size is related to the one closing the loop at zero instanton size exactly by the singular gauge transformation (4.16), which reads

$$
U=\frac{y^{m} \sigma^{m}}{|y|}
$$

This gauge transformation is large. While in the instanton literature it is merely employed as a computational trick to improve convergence of numerical calculations for large distance from the instanton core, in this setup it has physically observable consequences because the large distance behaviour is related to the conformal dimension of boundary operators. It also does not leave the global charges under $\mathrm{SU}(2)_{L} \times \mathrm{SU}(2)_{R} \times \mathrm{SU}(2)_{f}$

${ }^{*} \mathrm{SU}(2)_{R} \times \mathrm{SU}(2)_{f}$ is broken to $\operatorname{diag}\left[\mathrm{SU}(2)_{R} \times \mathrm{SU}(2)_{f}\right]$ except at zero and infinite Higgs VEV. 

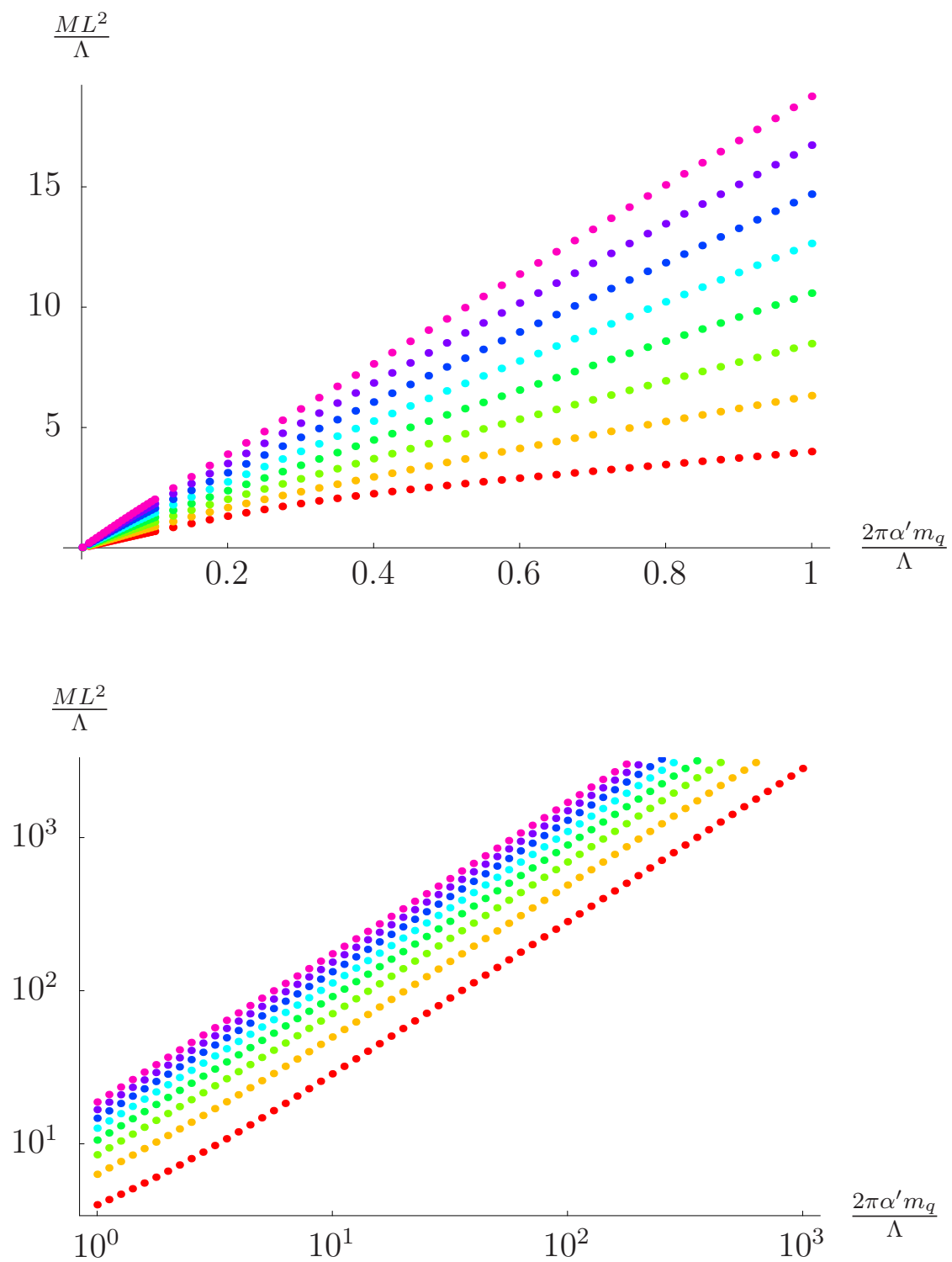

Figure 4.2: Numerical results for the meson mass spectrum as function of the quark mass. Both for $m_{q} / \Lambda \rightarrow 0$ and for $m_{q} / \Lambda \rightarrow \infty$, the curves become linear, however with different slope. The asymptotic slopes correspond to the constant values approached in Figure 4.1(b). 
invariant: Acting on the ansatz (4.30), the singular gauge transformation (4.16) yields

$$
\mathcal{A}_{\mu}^{(a)}=\xi_{\mu}(k) f(y) \mathrm{e}^{i k_{\mu} x_{\mu}}\left[\frac{y^{m} y^{n}}{y^{2}} \sigma^{m} T^{a} \bar{\sigma}^{n}\right] .
$$

The parenthesised expression should be the $\ell=2$ spherical harmonic. Due to $\sigma^{m} T^{a} \bar{\sigma}^{n}$ being traceless, there is indeed no singlet contribution. Moreover a spherical harmonic should be independent of $|y|$ as is true for $\frac{y^{m} y^{n}}{y^{2}}$. With $\hat{g}^{i j}$ the metric on the three sphere it holds

$$
\partial_{m} \partial_{m} \mathcal{Y}^{\ell}=y^{-2} \hat{\nabla}_{i} \hat{g}^{i j} \hat{\nabla}_{j} \mathcal{Y}^{\ell}=-\ell(\ell+2) y^{-2} \mathcal{Y}^{\ell}
$$

which is also satisfied by (4.45).

\subsubsection{Scalar Fluctuations}

The mesons arrange themselves in massive $\mathcal{N}=2$ multiplets, some of which are obtained by different, scalar ansätze for the gauge fluctuations (4.30). In addition, there arise mesons from fluctuations of the scalars in (4.27). For these the equation of motion reads

$$
H \partial_{\mu} \partial_{\mu} \Phi+\mathcal{D}_{m} \mathcal{D}_{m} \Phi=0
$$

where

$$
\begin{aligned}
\mathcal{D}_{m} \mathcal{D}_{m} \Phi= & \partial_{m} \partial_{m} \phi+\left[A_{m}^{\text {inst }}, \partial_{m} \Phi\right] \\
& +\partial_{m}\left[A_{m}^{\text {inst }}, \Phi\right]+\left[A_{m}^{\text {inst }},\left[A_{m}^{\text {inst }}, \Phi\right]\right]
\end{aligned}
$$

which coincides with the equation of motion for the gauge field (4.36) except for the vector index present. Therefore the same ansatz up to a polarisation vector

$$
\Phi^{(a)}=f(\tilde{y}) \mathrm{e}^{i k_{\mu} x_{\mu}} T^{a}
$$

yields exactly the same radial differential equation (4.39) and the same mass spectrum, Figure 4.1.

The scalar fluctuations (4.49) are dual to the descendant $Q Q\left(q_{i} \bar{q}^{i}\right)$ of the scalar bilinear $q_{i} \bar{q}^{i}$, which has conformal dimension $\Delta=3$. At $\Lambda=0$ 
the scalar bilinear is in the $(0,0)$ representation of the unbroken $\mathrm{SU}(2)_{L} \times$ $\mathrm{SU}(2)_{R}$ symmetry. 
If little else, the brane is an educational toy.

Tom Robbins (up to a small typo)

\section{Chapter 5}

\section{Heavy-Light Mesons}

$\S 5.1$ Heavy-Light Mesons in $\mathrm{AdS}_{5} \times \mathrm{S}^{5}, 74 . \quad \S 5.2$ Dilaton Flow Geometries, 80. §5.2.1 Gubser's Dilaton Deformed Geometry, 81. §5.2.2 Constable-Myers' Background, 85. §5.3 Bottom Phenomenology, 88.

This Chapter is similar in spirit to the D3-D7 systems discussed so far, though different in implementation. The reason is that while fundamental fields are still assumed to arise from D7 branes in a - possibly deformedAdS space, the requirement to describe quarks of vastly different mass, as needed for heavy-light mesons, makes those quarks arising from a stack of coincident D7-branes being no longer a good approximation. In this regard, heavy-light mesons are intrinsically stringy and cannot be captured by the DBI techniques discussed in the previous Chapters. Unfortunately as full quantised string theory on AdS is not well understood, the question arises of how to transfer such features into a supergravity framework.

Here idealised heavy-light mesons will be considered, composed of a massless and a very massive quark, such that in an appropriate background, the light quark may exhibit dynamical chiral symmetry breaking, while the heavy quark does not. For now, let us stick with the AdS case. Clearly the geometric picture is that of two parallel (probe) D7-branes in a background determined by a stack of D3-branes. The different quark 
masses correspond to the two different separations of the D7-branes from the D3 stack. Strings describing heavy-light mesons now differ from lightlight and heavy-heavy ones, whose ends are attached to the respective same brane, by being stretched between the two different D7-branes. In the limit where the heavy quark is much heavier than the light quark, henceforth called large separation limit, the string becomes very long and admits a classical description.

effective To obtain a description both simple and similar to the examples studied point-particle so far, the ansatz of a rigid non-oscillating string is chosen that moves in action the AdS radial direction along the D7-branes, with the essential assumption that oscillations and longitudinal movement are suppressed in the large separation limit.* Integration of the Polyakov action along the string can then be performed, yielding effectively a centre-of-mass movement weighted by a factor from averaging over the geometry between the two D7s. To obtain a field equation, naïve quantisation is performed, which results in a modified Klein-Gordon equation. (In a Minkowski space, this procedure yields the conventional, unmodified Klein-Gordon equations.) After the AdS case, the discussion will be moved on to the dilaton deformed background by Gubser introduced in Chapter 3 and a similar background by Constable-Myers. Both exhibit chiral symmetry breaking. While these are known to be far from perfect QCD gravity duals, experience shows that even simple holographic models can reproduce measured mass values with an accuracy of 10-20\%. Assuming the two respective quark flavours associated to the D7-branes being up and bottom, the mass of the rho $(u \bar{u})$ and upsilon $(b \bar{b})$ meson can be used to fix all scales in the theory and yield a numerical prediction for the B meson mass, which indeed is less than $20 \%$ from the experimental value.

\subsection{Heavy-Light Mesons in $\mathrm{AdS}_{5} \times \mathrm{S}^{5}$}

As shown in Chapter 2, quarks can be introduced into the AdS/CFT correspondence by augmenting the D3 stack with a stack of probe D7-branes

*On the field theory side at large separation; i.e. large quark mass $m_{H}$, effects distinguishing vector from scalar mesons are suppressed by $m_{H}^{-1}$. Indeed the formalism described here is not capable of capturing such a difference and meson masses are thus manifestly degenerate. 


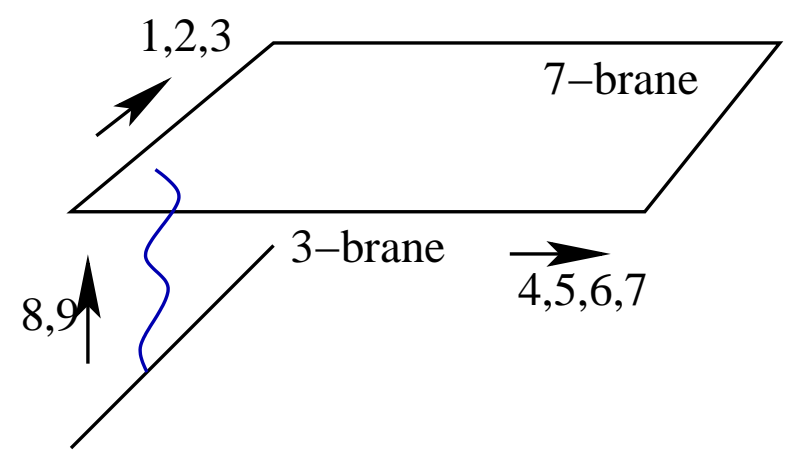

Figure 5.1: The geometry of the D3-D7 system under consideration [2].

[20]. The backreaction of the $N_{f}$ D7-branes on the $\mathrm{AdS}_{5} \times \mathrm{S}^{5}$ geometry (2.1) formed by the $N_{c}$ D3-branes may be neglected as long as $N_{f} \ll N_{c}$; i.e. $N_{f}$ is kept fixed in the 't Hooft limit.

$$
d s^{2}=\frac{r^{2}}{L^{2}} \eta_{\mu \nu} d x^{\mu} d x^{\nu}+\frac{L^{2}}{r^{2}} d r^{2}+L^{2} d \Omega_{5}^{2}
$$

This corresponds to the quenched approximation of lattice gauge theory on the field theory side. The D7-branes wrap an $\mathrm{AdS}_{5} \times \mathrm{S}^{3}$ geometry when coincident with the D3s. When separated the corresponding $\mathcal{N}=2$ hypermultiplet acquires a mass and the D7-branes wrap a geometry

$$
d s^{2}=\frac{y^{2}+\tilde{m}_{q}^{2}}{L^{2}} \eta_{\mu \nu} d x^{\mu} d x^{\nu}+\frac{L^{2}}{y^{2}+\tilde{m}_{q}^{2}} d y^{2}+\frac{L^{2} y^{2}}{y^{2}+\tilde{m}_{q}^{2}} d \Omega_{3}^{2},
$$

which is only asymptotically $\mathrm{AdS}_{5} \times \mathrm{S}^{3}$ and does not fill the complete $\mathrm{AdS}_{5}$ background, but instead terminates from the five-dimensional point of view and drops from the IR dynamics. This configuration is shown in Figure 5.1. The meson spectrum can be determined analytically [24] and spectrum the degenerate mass of the scalar and pseudoscalar meson is given by

$$
M_{s}^{2}=\frac{4 \tilde{m}_{q}^{2}}{L^{4}}(n+\ell+1)(n+\ell+2)
$$

These mesons are build up from quarks carrying all the same mass; two flavours i.e. they form "light-light" or "heavy-heavy" mesons depending on the distance $\tilde{m}_{q}=\left(2 \pi \alpha^{\prime}\right) m_{q}$ between the D7-branes and the D3 stack. When considering two D7-branes with different distances $\tilde{m}_{L}$ and $\tilde{m}_{H}$ to the 


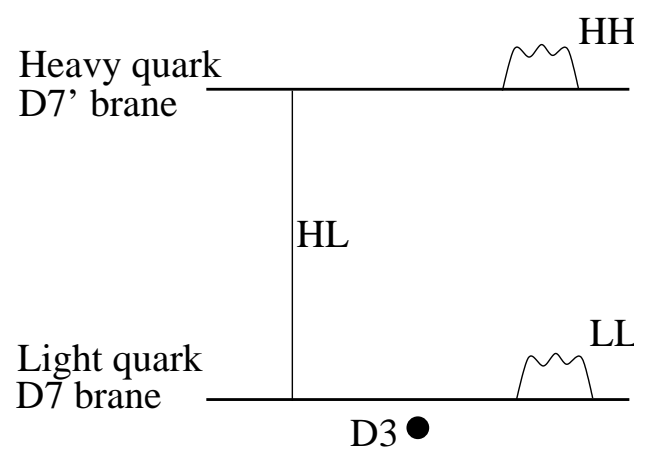

Figure 5.2: The brane configuration including both a heavy and a light quark. The 77 and $7^{\prime} 7^{\prime}$ strings are holographic to light-light and heavyheavy mesons respectively. Heavy-light mesons are described by strings between the two D7-branes.

D3 stack, there are accordingly two towers of mesons $M_{H}$ and $M_{L}$ whose lightest representatives have a mass ratio of $\frac{m_{L}}{m_{H}}$ and which come from strings having attached both ends to the same brane. The configuration is shown in Figure 5.2. Strings stretched between the two branes should then form a set of mesons composed of a heavy and a light quark.

In the limit $m_{H} \gg m_{L}$ the string becomes very long and will be assumed to be in the semi-classical limit, where quantum effects to the unexcited string can be neglected. The string described here will therefore approximate above mesons, which by construction will be degenerate.

Polyakov

The gauge-fixed Polyakov action will be taken as a starting point

$$
S_{P}=-\frac{T}{2} \int d \sigma d \tau G_{\mu \nu}\left(-\dot{X}^{\mu} \dot{X}^{\nu}+X^{\prime \mu} X^{\prime \nu}\right)
$$

such that the constraints

$$
G_{\mu \nu} \dot{X}^{\mu} X^{\prime \nu}=0, \quad G_{\mu \nu}\left(\dot{X}^{\mu} \dot{X}^{\nu}+X^{\prime \mu} X^{\nu}\right)=0
$$

have to be taken into account.

The two D7-branes are assumed to be separated from the D3 stack in the same direction $\theta=0$; i.e. the string connecting them will obey $\sigma=z$, where $\sigma$ is the spatial world sheet coordinate and $z \mathrm{e}^{i \theta}=z^{9}+i z^{8}$. While the string will be allowed to move along the world volume of the D7s, it shall be stiff such that integration over $\sigma$ can be performed to generate an effective point particle action. With the embedding 


$$
X^{A}=\left(x^{\mu}(\tau), y^{m}(\tau), z^{8}=0, z^{9}=\sigma\right)
$$

which implies $\dot{X}^{a} X_{a}^{\prime}=0$ automatically, and the $\mathrm{AdS}_{5} \times \mathrm{S}^{5}$ geometry (5.1), the Polyakov action reads

$$
S_{P}=-\frac{T}{2} \int d \tau \int_{\tilde{m}_{L}}^{\tilde{m}_{H}} d \sigma\left[-\frac{y^{2}+\sigma^{2}}{L^{2}} \dot{x}^{\alpha} \dot{x}_{\alpha}-\frac{L^{2}}{\left(y^{2}+\sigma^{2}\right)} \dot{y}^{i} \dot{y}_{i}+\frac{L^{2}}{\left(y^{2}+\sigma^{2}\right)}\right],
$$

where $y \equiv|y| \equiv \sqrt{\sum_{i=4,5,6,7}\left(y^{i}\right)^{2}}$. Integrating over $\sigma$ yields

$$
S_{P}=-\frac{T}{2} \int d \tau\left[-f(y) \dot{x}^{2}-g(y) \dot{y}^{2}+g(y)\right]
$$

with (choosing $\tilde{m}_{L}=0$ )

$$
f(y)=\frac{1}{L^{2}}\left(y^{2} \tilde{m}_{H}+\frac{1}{3} \tilde{m}_{H}^{3}\right), \quad g(y)=\frac{L^{2}}{y} \arctan \frac{\tilde{m}_{H}}{y} .
$$

The remaining constraint equation $G_{\mu \nu}\left(\dot{X}^{\mu} \dot{X}^{\nu}+X^{\prime \mu} X^{\nu}\right)=0$ is

$$
\frac{y^{2}+\sigma^{2}}{L^{2}} \dot{x}^{\alpha} \dot{x}_{\alpha}+\frac{L^{2}}{\left(y^{2}+\sigma^{2}\right)} \dot{y}^{i} \dot{y}_{i}+\frac{L^{2}}{\left(y^{2}+\sigma^{2}\right)}=0,
$$

which gives

$$
\begin{aligned}
\frac{1}{f(y)} p_{x}^{2} & +\frac{1}{g(y)} p_{y}^{2}+T^{2} g(y)=0 \\
p_{x}^{\alpha} & :=\frac{\partial \mathscr{L}}{\partial \dot{x}_{\alpha}} \\
p_{y}^{i} & :=\frac{\partial \mathscr{L}}{\partial \dot{y}_{i}}
\end{aligned}
$$

when integrating over $\sigma$. The same calculation for Minkowski space gives $f(y)=g(y)=\tilde{m}_{H}$, such that one obtains $E^{2}=m^{2}+p^{2}$. For AdS space the mass $m$ depends on the position of the string $y$ via the factors $f(y)$ and $g(y)$, which average over the geometry between the two D7-branes.

For the quantisation prescription $p \mapsto-i \partial$, the following modified equation of motion 

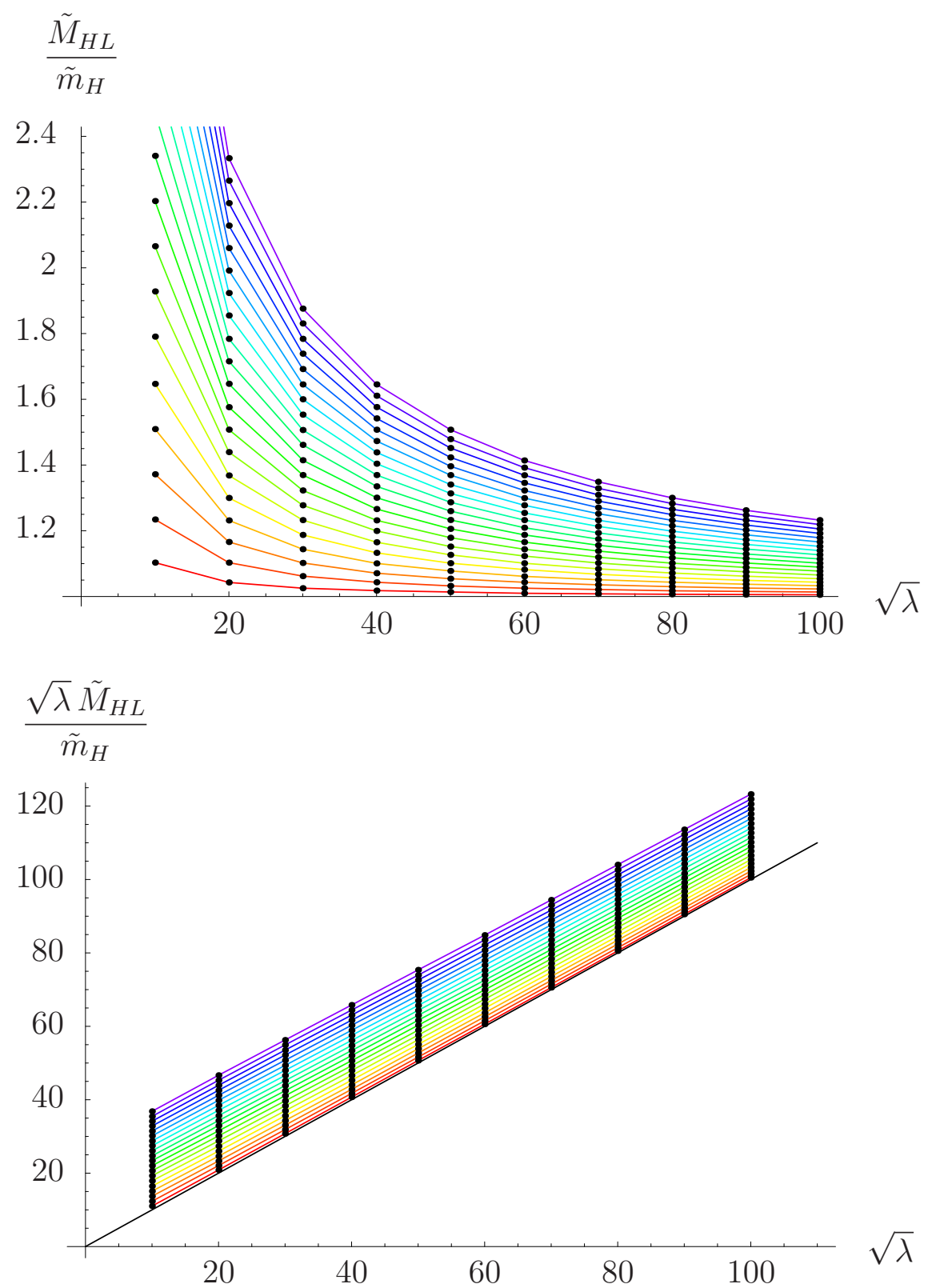

Figure 5.3: The mass ratio of the heavy-light meson and the heavy quark mass (the light quark is taken to be massless) as a function of the 't Hooft coupling for the AdS background. In the large $\lambda$ limit, $M_{H L} L^{2} /\left(2 \pi \alpha^{\prime} m_{H}\right)$ behaves as $1+$ const. $/ \sqrt{\lambda}+\mathcal{O}\left(\lambda^{-1}\right)$. The black line in the second plot corresponds to $M_{H L} L^{2}=\left(2 \pi \alpha^{\prime}\right) m_{H}$. 


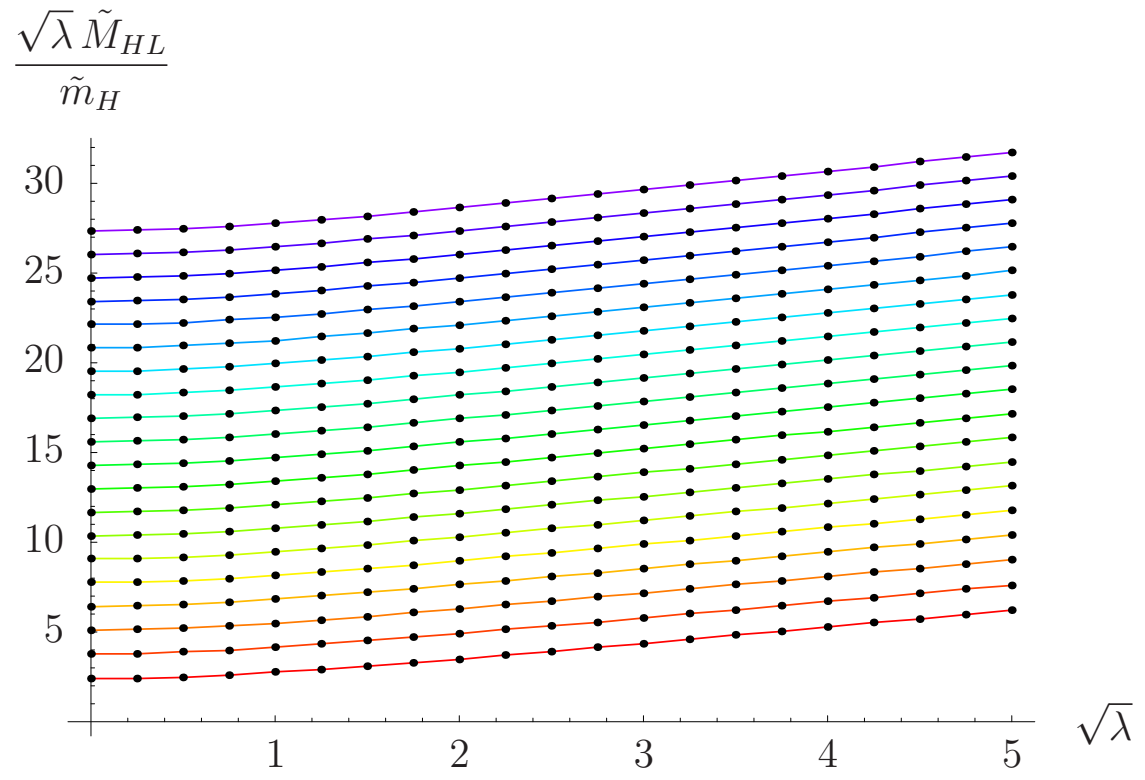

Figure 5.4: The heavy-light meson spectrum in AdS for small 't Hooft coupling with vanishing mass for the light quark. The mass ratio behaves as const. $/ \sqrt{\lambda}+\mathcal{O}(\lambda)$. Note however that the supergravity approximation is not reliable in this regime.

Klein-Gordon equation is obtained

$$
\left[\square_{x}^{2}+\frac{f(y)}{g(y)} \nabla_{y}^{2}-T^{2} g(y) f(y)\right] \phi(\vec{x}, \vec{y})=0
$$

The usual procedure for this kind of equations is to find the correct background solution, which by assumption only depends on the radial direction $y$ and find fluctuations about this solution. By a separation ansatz these fluctuations can be seen to be a plain wave in the $x$ direction and spherical harmonics in the angular coordinates $\Omega_{3}\left(y^{4,5,6,7}\right)$. The remaining equation for the radial coordinate $y$ often has to be solved numerically.

In the UV limit $y \rightarrow \infty$, (5.12) is dominated by the Laplace operator in the $y$ directions due to $\frac{f}{g} \sim y^{4}$ and $f g \rightarrow 1$, such that

$$
\nabla_{y}^{2} \phi=0
$$

When $\phi$ only depends on $y$, the solution has the form required to couple 
to the VEV and source of a heavy-light quark bilinear $\bar{\psi}_{H} \psi_{L}$.

$$
\phi(y \rightarrow \infty)=\tilde{m}_{H L}+\frac{c_{H L}}{y^{2}}+\ldots
$$

trivial vacuum However there are no heavy-light mass mixing term and no heavy-light bilinear condensate in QCD, so $\phi(y) \equiv 0$ is chosen.

Assuming a singlet under $\mathrm{SU}(2)_{L} \times \mathrm{SU}(2)_{R}$, the ansatz for linearised fluctuations about above vacuum solution reads

$$
\phi=0+h(y) \mathrm{e}^{i k \cdot x}, \quad M_{H L}^{2}=-k^{2},
$$

where $h(y)$ shall be regular in the IR and normalisable $h(y \rightarrow \infty) \sim y^{-2}$. Only for a discrete set of values for $M_{H L}$ this requirement can be satisfied. For numerics it is convenient to employ rescaled coordinates $y=\tilde{m}_{H} \tilde{y}$, such that equation (5.12) reads

$$
\left[\frac{\pi}{\lambda} \frac{\tilde{y}^{3}+\frac{\tilde{y}}{3}}{\arctan \frac{1}{\tilde{y}}} \nabla_{\tilde{y}}^{2}+\left(\tilde{y}+\frac{1}{3 \tilde{y}}\right) \arctan \frac{1}{\tilde{y}}+\frac{M^{2} L^{4}}{\tilde{m}_{H}^{2}}\right] h(\tilde{y})=0
$$

The 't Hooft coupling $\lambda$ arises from $R^{4} /\left(2 \pi \alpha^{\prime}\right)=g_{s} N_{c} / \pi$. The mass ratios yielding regular normalisable solutions to (5.16) have been plotted in Figures 5.3 and 5.4. It can be read off

$$
\frac{M_{H}}{m_{H}}=\frac{2 \pi \alpha^{\prime}}{L^{2}}\left[1+\frac{\text { const. }}{\sqrt{\lambda}}+\mathcal{O}\left(\lambda^{-1}\right)\right]
$$

In the large $\lambda$ limit, $\tilde{M}_{H L}=\tilde{m}_{H}$ is approached in agreement with the naivve expectation of the meson mass being equal to the string length times its tension. For comparison in Figure 5.4 the mass ratio is plotted for small values of the 't Hooft coupling, where supergravity is not a reliable approximation anymore.

\subsection{Dilaton Flow Geometries}

The $\mathcal{N}=2$ SYM considered so far provides a basis for studying meson spectra since it gives analytic expressions for solutions and masses consisting of identical quarks. However it does not capture a number of 
phenomenologically relevant features like chiral symmetry breaking since chiral symmetry breaking requires SUSY breaking. The setup discussed now improves at least in that regard by providing a simple geometry that describes a non-supersymmetric dual of a large $N_{c}$ QCD-like theory and thus exhibits dynamical chiral symmetry breaking.

The first background discussed is the dilaton deformed background by Gubser, which has been described in Chapter 3. It is demonstrated that the semi-analytic prediction of the AdS case is reproduced in the large heavy-quark limit. Then the same procedure is applied to the similar geometry of Constable and Myers, but it turns out that in this setup the heavy-light meson spectrum does not approach the AdS spectrum in a similar manner.

\subsubsection{Gubser's Dilaton Deformed Geometry}

Let me remind the reader that Gubser's geometry is given by, cf. (3.26),

$$
\begin{aligned}
d s_{10}^{2} & =g_{x x}(r) d x_{1,3}^{2}+g_{y y}(r)\left(d \vec{y}^{2}+d \vec{z}^{2}\right), \\
g_{x x}(r) & =\frac{r^{2}}{L^{2}} \sqrt{1-r^{-8}} \\
g_{y y}(r) & =g_{z z}=\frac{L^{2}}{r^{2}}, \\
\mathrm{e}^{\varphi} & =\mathrm{e}^{\varphi_{0}}\left(\frac{r^{4}+1}{r^{4}-1}\right)^{\sqrt{\frac{3}{2}}}, \\
r^{2} & =\vec{y}^{2}+\vec{z}^{2}
\end{aligned}
$$

where Einstein frame has been used and the coordinates have been rescaled such that infra-red singularity resides at $r=1$. The coordinates $y^{4,5,6,7}$ and $z^{8,9}$ are on equal footing and can be interchanged by $\mathrm{SO}(6)$ transformations until probe D7-branes, which break the $\mathrm{SO}(6)$ to $\mathrm{SO}(4) \times \mathrm{SO}(2)$, are introduced to obtain quarks. The D7-branes are embedded according 


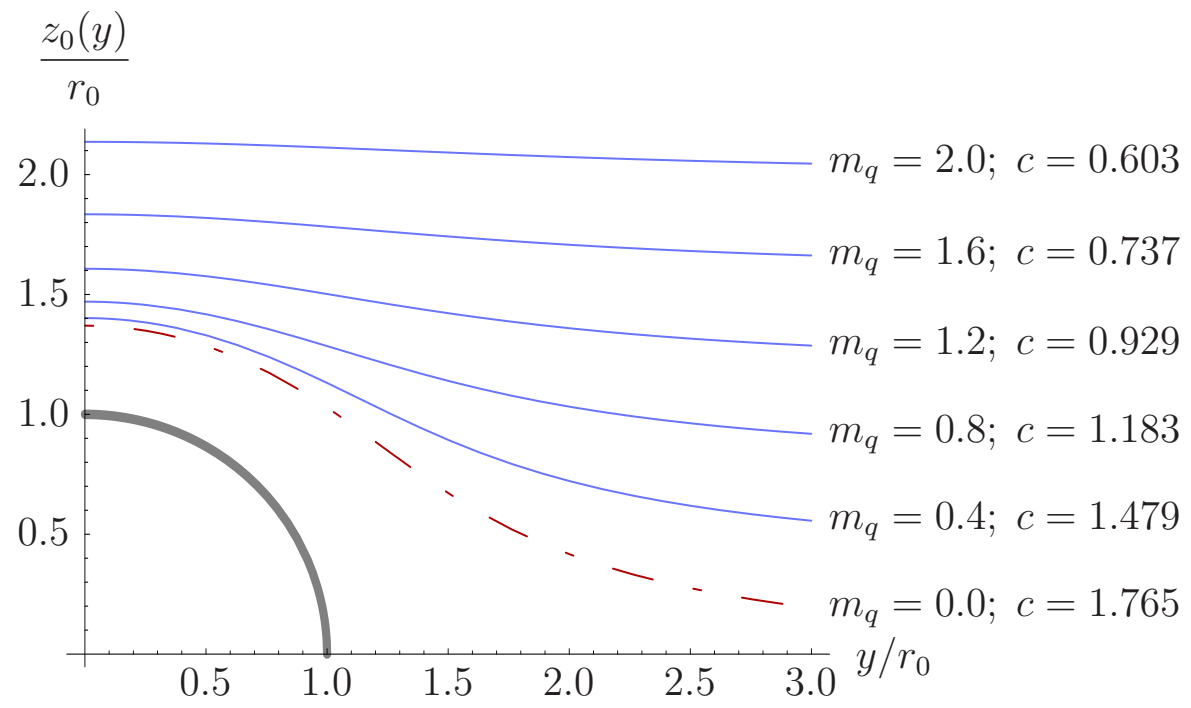

Figure 5.5: Chiral symmetry breaking embeddings in Gubser's background.

to $z=\left|z^{9}+i z^{8}\right|=z_{0}(y)$, which yields the following equation of motion

$$
\begin{gathered}
\frac{d}{d y}\left[\frac{y^{3} f}{\sqrt{1+z_{0}^{\prime}(y)^{2}}} z_{0}^{\prime}(y)\right]=y^{3} \sqrt{1+z_{0}^{\prime}(y)^{2}} \frac{\partial}{\partial z_{0}} f, \\
f=\frac{\left(r^{4}+1\right)^{(1+\Delta / 2)}\left(r^{4}-1\right)^{(1-\Delta / 2)}}{r^{8}}, \quad r^{2}=y^{2}+z_{0}(y)^{2}, \quad \Delta=\sqrt{6} .
\end{gathered}
$$

At large $y$, solutions to (5.19) take the form

$$
z_{0}=\frac{\tilde{m}_{q}}{r_{0}}+\frac{c}{r_{0}^{3} y^{2}}+\ldots
$$

which by standard AdS/CFT duality corresponds to a source of conformal dimension 1 and a VEV of conformal dimension 3 in the field theory. The former corresponds to the quark mass $m_{q}=\tilde{m}_{q} /\left(2 \pi \alpha^{\prime}\right)$ and describes the asymptotic separation $\tilde{m}_{q}$ of the D3 and D7-branes, the latter is the bilinear quark condensate $c \sim\langle\bar{\psi} \psi\rangle$. The factor of $r_{0}$, which gives the position of the singularity, arises from the coordinate rescaling used to remove $r_{0}$ from the metric and equations of motion.

regular embeddings

Requiring regularity in the IR by $\partial_{y} z_{0}(0)=0$ fixes the quark condensate as a function of the quark mass, see Section 3.6. Some regular solutions to 
(5.19) are plotted in Figure 5.5, which provide the D7 embeddings that are used as the boundary conditions for the heavy-light string in the following.

The Polyakov action (5.4), which due to being in string frame requires additional factors of $\mathrm{e}^{\varphi / 2}$, reads for this background

$$
S_{P}=-\frac{T}{2} \int d \tau \int_{z_{0}\left(m_{L}\right)}^{z_{0}\left(m_{H}\right)} d z_{0}\left[-\mathrm{e}^{\varphi / 2} g_{x x} \dot{x}^{\alpha} \dot{x}_{\alpha}-\mathrm{e}^{\varphi / 2} g_{y y} \dot{y}^{i} \dot{y}_{i}+\mathrm{e}^{\varphi / 2} g_{y y}\right]
$$

with the metric factors and dilaton from (5.18).

One obtains again an equation of motion of the form

$$
\left[\square_{x}^{2}+\frac{f(y)}{g(y)} \nabla_{y}^{2}-T^{2} g(y) f(y)\right] \phi(\vec{x}, \vec{y})=0,
$$

where the coefficients $f(y)$ and $g(y)$ this time are given by

$$
f(y)=\int_{z_{0}\left(m_{L}\right)}^{z_{0}\left(m_{H}\right)} d z_{0} \mathrm{e}^{\varphi / 2} g_{x x}, \quad g(y)=\int_{z_{0}\left(m_{L}\right)}^{z_{0}\left(m_{H}\right)} d z_{0} \mathrm{e}^{\varphi / 2} g_{y y} .
$$

The integration limits in (5.23); i.e. the positions of the D7-branes, are given by the solutions to (5.19), which are only known numerically, such that $f(y)$ and $g(y)$ also require numerics.

For an ansatz describing a field theoretic vacuum $\phi \equiv \phi_{0}(y)$, equation (5.22) has the same UV behaviour as the AdS case, $\phi_{0}(y \rightarrow \infty) \sim \tilde{m}_{H L}+$ $c_{H L} y^{-2}$, where $\tilde{m}_{H L}$ corresponds to heavy-light mass mixing term and $c_{H L}$ to a heavy-light quark condensate. Because both are absent in QCD, fluctuations about the trivial vacuum $\phi_{0}(y) \equiv 0$ are considered. Plot 5.6 fluctuation shows the mass spectrum of normalisable, regular solutions

$$
\delta \phi=\phi(y) \mathrm{e}^{i k \cdot x}
$$

as it can be obtained from

$$
\left[\frac{M_{H L}^{2}}{\Lambda^{2}}+\frac{\pi}{\lambda} \frac{\hat{f}(y)}{\hat{g}(y)} \nabla_{y}^{2}-g(y) f(y)\right] \phi(\vec{x}, \vec{y})=0
$$



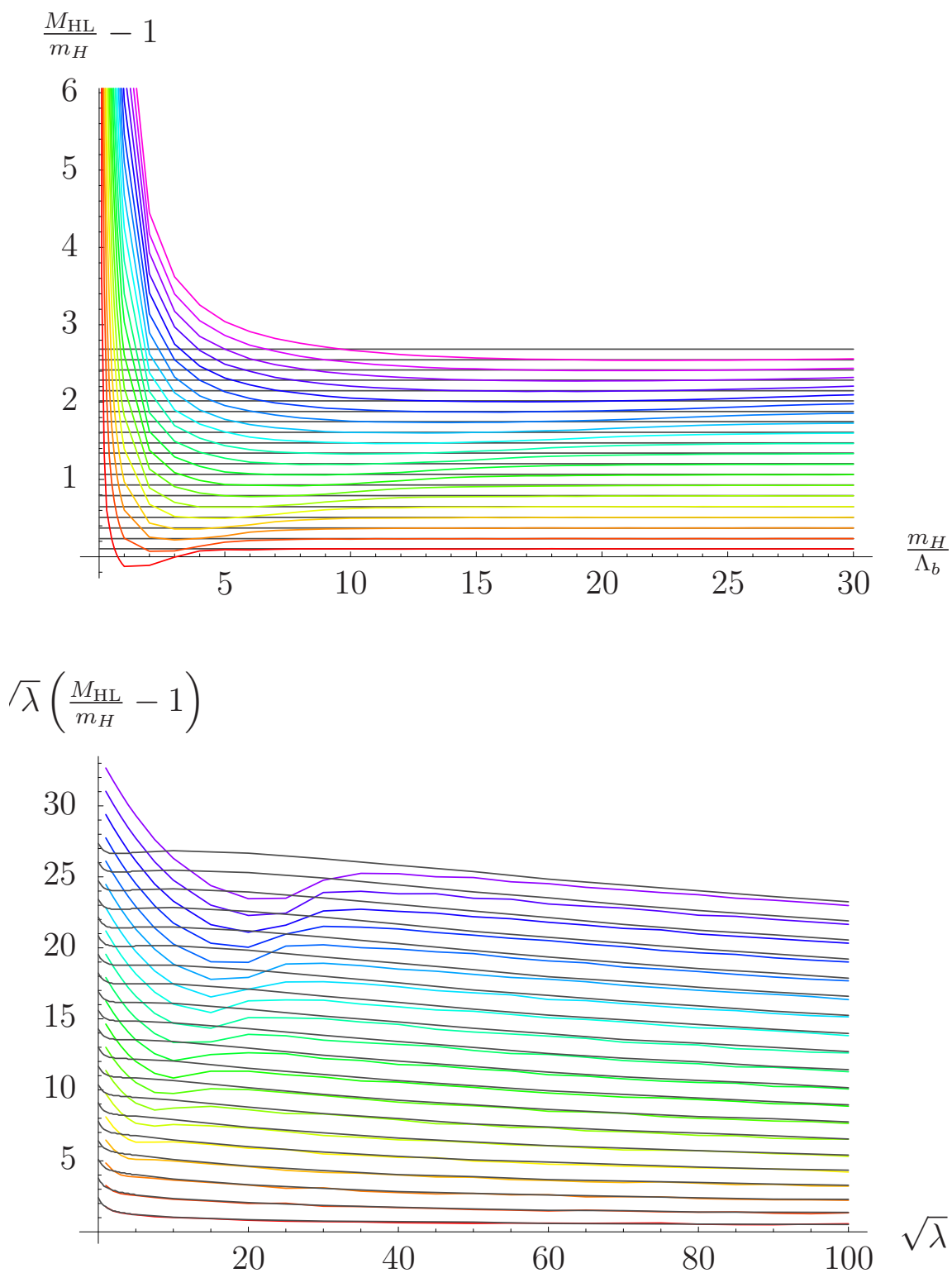

Figure 5.6: The binding energy of the heavy-light meson masses as a function of the heavy quark mass for $\lambda=100$ (first plot) and as a function of the 't Hooft coupling for $m_{H}=11.50 \Lambda$ (second plot). The respective AdS values are shown as gray lines in the background and are approached in the limit of large values of the heavy quark mass, while for small values effects of the chiral symmetry breaking are seen. 
with $\Lambda=r_{0} /\left(2 \pi \alpha^{\prime}\right)$ the QCD scale. $\hat{f}$ and $\hat{g}$ can be obtained from (5.23) by setting $L=1$. The light quark mass $m_{L}$ has been set to zero to describe a quark experiencing dynamical chiral symmetry breaking, while the large quark mass $m_{H}$ is varied.

The spectrum obtained is very similar to that of the AdS geometry. To make the deviations caused by the deformation more visible, the binding energy has been plotted. In Figure 5.6 it is shown for $\lambda=100$ as a function of the quark mass. It is also shown as a function of the 't Hooft coupling with the (for now arbitrary value of the) heavy quark mass $m_{H}=11.50 \Lambda$. The binding energy approaches its AdS values for $m_{H} \rightarrow \infty$, but highly SUSY restoration excited mesons converge more slowly. Both features can be understood from the spectrum of light-light/heavy-heavy mesons in Chapter 3. The higher the quark mass, the higher is the energy scale, where the brane "ends" and decouples from the spectrum. At high energies supersymmetry is restored and the light-light mesons become degenerate. While the effect is the same for the heavy-light mesons, that argument is not quite true anymore since the light quark has been set to be massless all the time- at least one end of the string stays close to IR region. However the centre of mass of the heavy-light string moves farther away from the interior of the space when the heavy quark mass grows. The effective averaging of the geometry in (5.23) takes into account more and more of the geometry far from the centre, which is nearly AdS.

At the same time highly excited mesons probe the IR more densely as has been seen in Section 3.8, so they require the string to be stretched much more to allow neglecting the vicinity of the singularity.

\subsubsection{Constable-Myers' Background}

The particular geometry considered here is a dilaton deformed AdS geometry introduced in [42], which has been employed by [82, 94] to describe chiral symmetry breaking in AdS/CFT. Like the background of the previous Section it is a warped $\mathrm{AdS}_{5} \times \mathrm{S}^{5}$ geometry with a running dilaton that preserves $\mathrm{SO}(1,3) \times \mathrm{SO}(6)$ isometry. 
The background is given by

$$
\begin{array}{rlrl}
d s^{2} & =H^{-1 / 2} X^{\delta / 4} d x_{1,3}^{2}+H^{1 / 2} X^{(2-\delta) / 4} Y\left(d \vec{y}^{2}+d \vec{z}^{2}\right), & \\
H & =X^{\delta}-1, & X & =\frac{r^{4}+b^{4}}{r^{4}-b^{4}}, \quad Y=\frac{r^{4}-b^{4}}{r^{4}}, \\
\mathrm{e}^{2 \varphi} & =\mathrm{e}^{2 \varphi_{0}} X^{\Delta}, \\
\delta & =\frac{L^{4}}{2 b^{4}}, & C_{(4)} & =H^{-1} d x_{0} \wedge \cdots \wedge d x_{3}, \\
\Delta^{2} & =10-\delta^{2},
\end{array}
$$

with $r^{2}=\vec{y}^{2}+\vec{z}^{2}$. $R$ and $b$ are free parameters and will be set to 1 for the numerics, since that allows to make contact with [82], where the same choice has been made. The authors of [82] embedded the D7-branes according to $z=\left|z^{9}+i z^{8}\right|=z_{0}(y)$ and obtained the following equation of motion

$$
\frac{d}{d y}\left[\frac{\mathrm{e}^{\varphi} \mathcal{G}\left(y, z_{0}\right)}{\sqrt{1+\left(\partial_{y} z_{0}\right)^{2}}}\left(\partial_{y} z_{0}\right)\right]=\sqrt{1+\left(\partial_{y} z_{0}\right)^{2}} \frac{\partial}{\partial z_{0}}\left[\mathrm{e}^{\varphi} \mathcal{G}\left(y, z_{0}\right)\right]
$$

where

$$
\mathcal{G}\left(y, z_{0}\right)=y^{3} \frac{\left(\left(y^{2}+z_{0}^{2}\right)^{2}+1\right)^{1+\Delta / 2}\left(\left(y^{2}+z_{0}^{2}\right)^{2}-1\right)^{1-\Delta / 2}}{\left(y^{2}+z_{0}^{2}\right)^{4}} .
$$

This is the same equation as (5.19) albeit with a free parameter $\Delta$, which in Gubser's geometry has the fixed value $\sqrt{6}$. The asymptotic behaviour and their field theoretic interpretation are the same as for Gubser's background and have been reviewed in the previous Section. Note however that only the particular combination $\mathrm{e}^{\varphi} \sqrt{-g}$ appearing in the equation for the vacuum embedding (5.27) coincides in both backgrounds. On the level of meson spectra, the results for light-light mesons are similar but not identical to those in Gubser's background.

Expanding the DBI action (1.15) to quadratic order in fluctuations (3.12) yields (3.19) for a vector meson ansatz, that is an ansatz of the form $A_{\mu}=\xi_{\mu} \delta \rho(y) \mathrm{e}^{i k \cdot x}, M_{\rho}^{2}=-k^{2}$ for the D7 gauge field. The vector meson radial equation (3.19) reads for the Constable-Myers background

$$
\partial_{y}\left(K_{1}(y) \partial_{y} \delta \rho(y)\right)+M_{\rho}^{2} K_{2}(y) \delta \rho(y)=0
$$



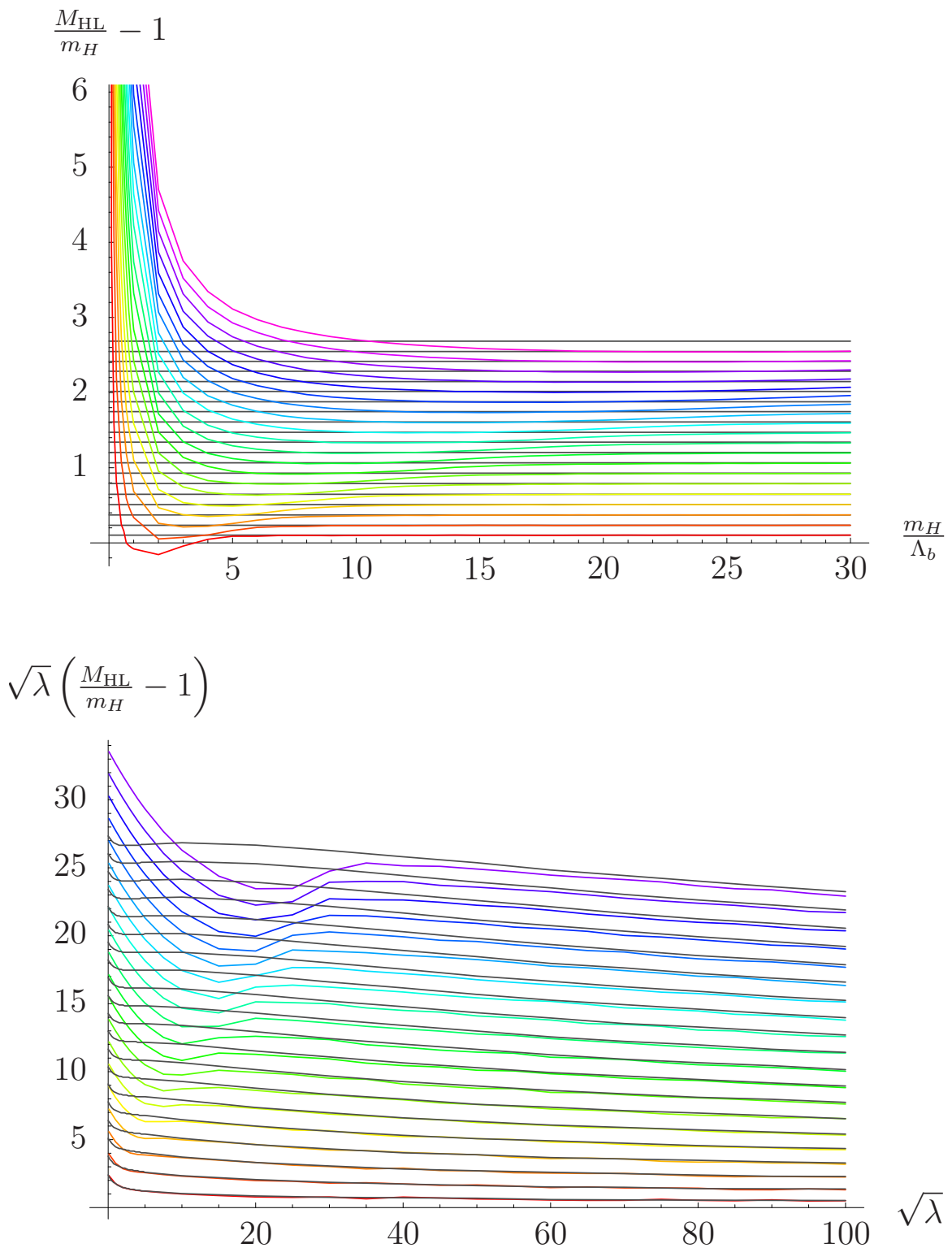

Figure 5.7: The binding energy of the heavy-light meson masses as a function of the heavy quark mass for $\lambda=100$ (first plot) and as a function of the 't Hooft coupling for $m_{H}=12.63 / \Lambda_{b}$ (second plot). The respective AdS values are shown as gray lines in the background and are approached in the limit of large values of the heavy quark mass, while for small values effects of the chiral symmetry breaking are seen. 
with

$$
K_{1}=X^{1 / 2} y^{3}\left(1+z_{0}^{\prime 2}\right)^{-1 / 2}, \quad K_{2}=H X^{1-\delta / 2} Y^{2} y^{3}\left(1+z_{0}^{\prime 2}\right)^{-1 / 2}
$$

and

$$
X=\frac{\left(y^{2}+z_{0}^{2}\right)^{2}+1}{\left(y^{2}+z_{0}^{2}\right)^{2}-1}, \quad Y=\frac{\left(y^{2}+z_{0}^{2}\right)^{2}-1}{\left(y^{2}+z_{0}^{2}\right)^{2}}
$$

The Polyakov action

$$
S_{P}=-\frac{T}{2} \int d \tau\left[-f(y) \dot{x}^{2}-g(y) \dot{y}^{2}+g(y)\right]
$$

preserves its AdS form but the coefficients are now

$$
\begin{aligned}
f(y) & =\int_{z_{0}\left(m_{L}\right)}^{z_{0}\left(m_{H}\right)} d z_{0}\left(X^{1 / 2}-1\right)^{-1 / 2} X^{\Delta+\frac{1}{8}}, \\
g(y) & =\int_{z_{0}\left(m_{L}\right)}^{z_{0}\left(m_{H}\right)} d z_{0} Y\left(X^{1 / 2}-1\right)^{1 / 2} X^{\Delta+\frac{3}{8}},
\end{aligned}
$$

with $X, Y$ defined in (5.31) and the integration limits are given by the solutions to (5.27).

Scalar fluctuations of the form $\phi=0+\delta \phi(y) \mathrm{e}^{i k \cdot x}$ yield

$$
\left[\frac{M_{H L}^{2}}{\Lambda_{b}^{2}}+\frac{\left(2 \pi \alpha^{\prime}\right)^{2}}{b^{4}} \frac{f(y)}{g(y)} \nabla_{y}^{2}-g(y) f(y)\right] \phi=0,
$$

with $\Lambda_{b}=b /\left(2 \pi \alpha^{\prime}\right)$ the QCD scale and $\left(2 \pi \alpha^{\prime}\right)^{2} / b^{4}=2 \pi \delta / \lambda$. For boundary conditions $\partial_{y} \delta \phi(0)=0$ and $\delta \phi(y \rightarrow \infty) \sim c y^{-2}$ equation (5.35) determines the meson spectrum. Since it is very similar to the AdS spectrum, the binding energy, which demonstrates the deviations more clearly, has been plotted in Figure 5.7 for massless light quark.

\subsection{Bottom Phenomenology}

There has been a number of attempts to apply holographic methods to phenomenological models [95, 96], even for the Constable-Myers background 


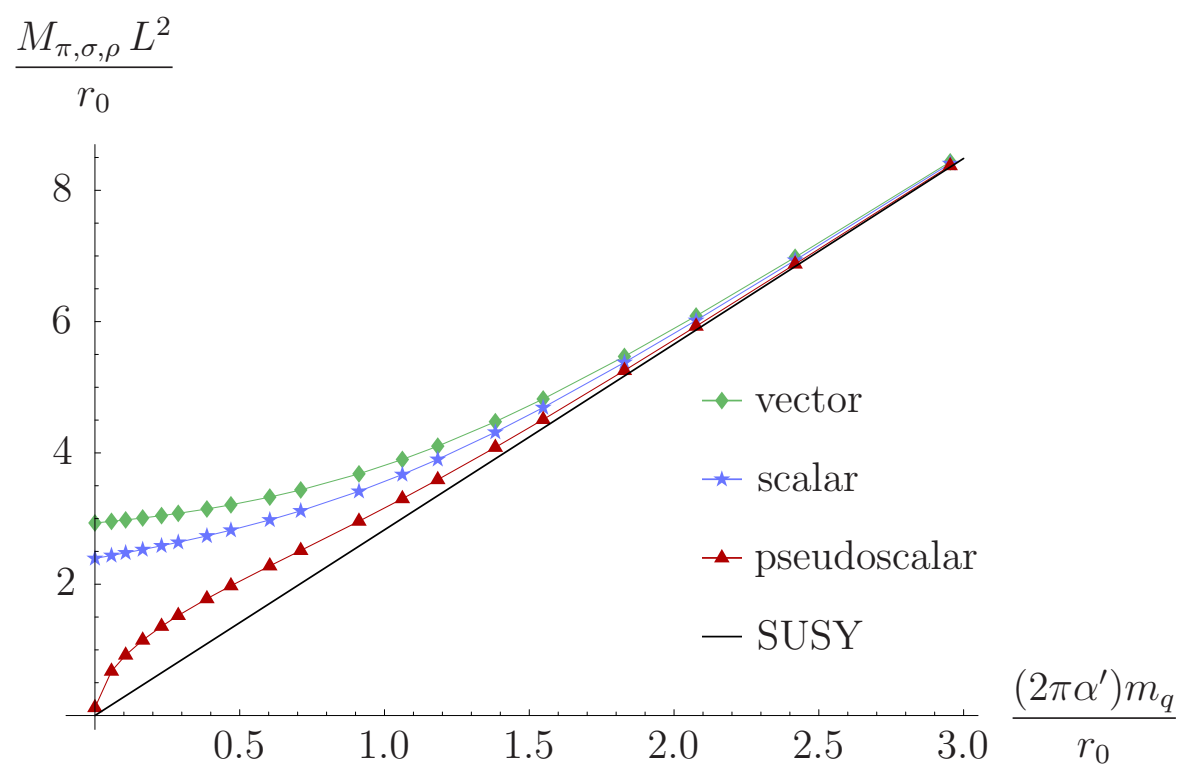

Figure 5.8: Lightest pseudoscalar, scalar and vector mesons in Gubser's dilaton deformed geometry. The vector mode for the massless quark is interpreted as a Rho meson, while for the heavy quark mass it yields the the Upsilon. See also Section 3.7.

of the previous Section [97], successfully reproducing light quark meson data with an accuracy better than $20 \%$. That shall be motivation enough to compare the heavy-light spectra calculated here with the bottom quark sector of QCD; i.e. the massless quark in the setup above will be assumed to play the rôle of an up quark, while the heavy quark, which will lie in the AdS-like region, will be interpreted as a bottom quark.

In that regime supersymmetry will be restored and the field theory will shortcomings be strongly coupled even though QCD dynamics should be perturbative at this energy scale. These are respective consequences of the background being too simple (though a background exhibiting separation of scales is not known yet) and an intrinsic feature of the SUGRA version of AdS/CFT that can only be overcome by a full string treatment, which is currently out of reach.

The scales of the theory will be fixed by identifying the mass of the lowest vector meson mode with the Rho and Upsilon mesons, which are chosen as input data since they are less sensitive to the light quark mass than the pseudoscalar modes roughly corresponding to the Pion, cf. Fig- 


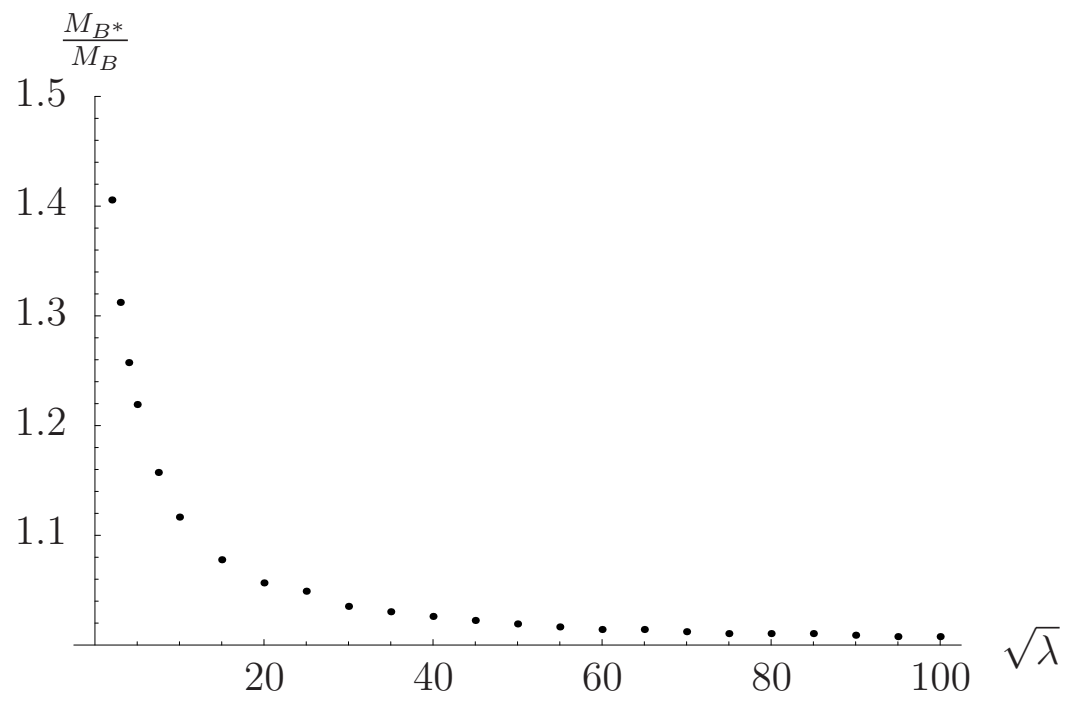

Figure 5.9: Ratio of the mass of the lowest and first excited heavy-light meson mode for the Gubser and Constable-Myers background. (They really do look exactly the same, since the different units expressing the different dependence on the respective deformation parameter cancel in the ratio.) For large 't Hooft parameter the ratio approaches 1, while the physical $\mathrm{B} / \mathrm{B}^{*}$ ratio (which is 1.01 ) is reached at $\lambda \approx 82$.

ure 5.8 and Section 3.7 for details.

From Figure 5.8 the $\rho$ mass for Gubser's background is read off to be $M_{\rho} L^{2} / r_{0}=2.93$. Preserving the physical ratio

$$
M_{\Upsilon} / M_{\rho}=9.4 \mathrm{GeV} / 770 \mathrm{MeV}
$$

the $\Upsilon$ mass has to be $M_{\Upsilon} L^{2} / r_{0}=35.8$ and the heavy quark mass can be read off to be $m_{b}=12.7 \Lambda$.

The 't Hooft parameter can be determined from the physical ratio of the mass of the Rho and the B meson by searching for the value of $\lambda$ for which the numerical value of the lowest heavy-light excitation satisfies

$$
\left(\frac{M_{B}}{M_{\rho}}\right)^{\text {phys }}=\left(\frac{M_{H L}(\lambda)}{\Lambda}\right)^{\text {num }}\left(\frac{r_{0}}{M_{\rho} L^{2}}\right)^{\text {num }} \sqrt{\frac{\lambda}{\pi}} .
$$

Unfortunately this yields a value of the 't Hooft coupling of $\lambda=2.31$. As can be seen in Figure 5.9 the mass ratio of the predicted B and $\mathrm{B}^{*}$ meson reaches its physical value of approximately 1.01 only for very large 
$\lambda$. Identifying $M_{H L}$ with the physical quark mass $M_{B}=5279 \mathrm{MeV}$, one obtains a QCD scale of $225 \mathrm{MeV}$.

With respect to the $\mathrm{B}$ mass ratio, the situation is slightly better for the background by Constable and Myers, where the same procedure yields a prediction of $\lambda=5.22$. While it is not clear if this value is sufficient for the large $\lambda$ approximation inherent in the employed formulation of the AdS/CFT correspondence, it gives a prediction for $M_{B^{*}}=6403 \mathrm{MeV}$, which is $20 \%$ larger than the measured value of $5325 \mathrm{MeV}$. Again a much larger value of the 't Hooft coupling would be required to achieve a better agreement. For the QCD scale on obtains $\Lambda_{b}=340 \mathrm{MeV}$, which is a little too high. With $m_{H}=12.63 \Lambda_{b}$ the physical b quark mass is predicted to be $4294 \mathrm{MeV}$.

The overall agreement with experiment is far from perfect. However this does not come as a surprise since the b quark mass $\left(m_{b} \approx 12 \Lambda\right.$ in both backgrounds) is far in the supersymmetric regime: Restoration of supersymmetry takes place approximately at $m_{q} \approx 1.5 \Lambda$ as can be seen in Figure 5.8. In other words a string connecting a brane describing a light quark and this "b quark" has about $80 \%$ of its length in the supersymmetric region, which is a good approximation of pure AdS. The only way to improve this situation would be to use a (yet unknown) background that allows to separate the SUSY breaking scale from the QCD scale. 



\section{Part II}

\section{Space-time Dependent Couplings}



Supersymmetry is the greatest invention since the wheel.

A. Oop, "A supersymmetric version of the leg", Gondwansaland predraw, to be discovered [98]

\section{Chapter 6}

\section{Supergravity Overview}

$\S 6.1$ Conventions, 96. §6.2 Superspace Supergravity, 102. $§ 6.3$ Non-minimal Supergravity, 104. §6.3.1 Algebra and Bianchi identities, 105. §6.3.2 Partial Integration, 106. §6.3.3 Superdeterminant, 107. §6.3.4 Super-Weyl Transformations, 108. §6.3.5 Prepotentials, 109. §6.4 Minimal Supergravity, 110. §6.4.1 Algebra and Bianchi Identities, 110. §6.4.2 Chiral Projector and d'Alembertian, 111. §6.4.3 Super-Weyl Transformations, 112. §6.4.4 Chiral Representation and Integration Rule, 114. §6.5 Component Expansion, 115. §6.5.1 Superfields and First Order Operators, 115. §6.5.2 Supergravity Fields, 117. §6.5.3 Full Superspace Integrals, 119.

The second part of this thesis is devoted to the discussion of the conformal anomaly in supersymmetric field theories, in particular supersymmetric Yang-Mills theories.

The approach chosen is an extension to superfields of the space-time dependent coupling techniques Osborn [48] applied to non-supersymmetric theories coupled to a gravity background in order to give an alternative proof of Zamolodchikov's $c$-theorem, cf. Chapter 7. Consequently a coupling to supergravity will have to be considered and its superfield formulation shall be reviewed in this Chapter.

In Chapter 8 a discussion of the supersymmetric conformal anomaly will be given. 


\subsection{Conventions}

To establish notations, a few basic ingredients for supersymmetry are reviewed in the shortest possible manner. Throughout this part, a dotted/undotted Weyl spinor notation is being used.

The simplest double covering representation of the Lorentz group can be constructed as follows. An arbitrary vector $v^{\alpha \dot{\alpha}}$ transforms under a Lorentz transformation $\Lambda_{b}^{a} \in \mathrm{SO}(1,3)$ according to

$$
x^{a} \mapsto x^{a}=\Lambda_{b}^{a} x^{b} .
$$

double covering The double covering group $\mathrm{SL}(2, \mathbb{C})$ transforms the same vector according to

$$
\sigma_{a}^{\alpha \dot{\alpha}} x^{a} \mapsto\left(U^{\alpha}{ }_{\beta} \sigma_{a}^{\beta \dot{\beta}} U^{\dagger \dot{\alpha}}\right) x^{a} \equiv \sigma_{a}^{\alpha \dot{\alpha}} x^{\prime a},
$$

with $U$ the element of the double covering group chosen such that $x^{\prime a}$ coincides with the definition (6.1). The matrices $\sigma^{a}:=(\mathbb{1}, \vec{\sigma})$ are the Pauli matrices augmented by the unity matrix. As an aside, the " 1 to 2 " relation of the two representations can be easily seen from the fact that for any $U$ being a solution to $\left(U^{\alpha}{ }_{\beta} \sigma_{a}^{\beta \dot{\beta}} U^{\dagger \dot{\alpha}} \dot{\beta}\right)=\sigma_{b}{ }^{\alpha \dot{\alpha}} \Lambda^{b}{ }_{a},-U$ is also a solution. symplectic metric The group $\operatorname{SL}(2, \mathbb{C})$ leaves invariant the antisymmetric tensors $\varepsilon_{\alpha \beta}$ and $\varepsilon_{\dot{\alpha} \dot{\beta}}$, defined by

$$
\varepsilon_{12}=\varepsilon_{\dot{1} \dot{2}}=-1, \quad \quad \varepsilon^{12}=\varepsilon^{\dot{i} \dot{2}}=1,
$$

where the epsilon symbols with raised indices constitute the respective inverse matrices by $\varepsilon^{\alpha \beta} \varepsilon_{\beta \gamma}=\delta_{\gamma}^{\alpha}$. Since for any element $U$ of $\operatorname{SL}(2, \mathbb{C})$ it holds the relation $\varepsilon^{\alpha \beta}=\varepsilon^{\gamma \delta} U_{\gamma}{ }^{\alpha} U_{\delta}^{\beta}$, the combination $\varepsilon^{\alpha \beta} \psi_{\alpha} \psi_{\beta}$ is invariant under $\psi_{\alpha} \mapsto U_{\alpha}{ }^{\beta} \psi_{\beta}$ and therefore a Lorentz scalar. In other words, the epsilon matrices can be used to obtain contragradiently transforming representations according to

$$
\begin{array}{ll}
\psi^{\alpha}=\varepsilon^{\alpha \beta} \psi_{\beta}, & \psi_{\alpha}=\varepsilon_{\alpha \beta} \psi^{\beta}, \\
\bar{\psi}^{\dot{\alpha}}=\varepsilon^{\dot{\alpha} \dot{\beta}} \bar{\psi}_{\dot{\beta}}, & \bar{\psi}_{\dot{\alpha}}=\varepsilon_{\dot{\alpha} \dot{\beta}} \bar{\psi}^{\dot{\beta}} .
\end{array}
$$


For the sake of brevity, an indexless notation is often employed for con- indexless tracted adjacent objects, where different conventions are being used for notation dotted and undotted indices,

$$
\psi \chi:=\psi^{\alpha} \chi_{\alpha}, \quad \bar{\psi} \bar{\chi}=\bar{\psi}_{\dot{\alpha}} \bar{\chi}^{\dot{\alpha}} .
$$

This particular choice has the advantage that $\overline{\psi \chi}=\bar{\psi} \bar{\chi}$.

It is common to introduce

$$
x^{\alpha \dot{\alpha}}:=\tilde{\sigma}_{a}^{\alpha \dot{\alpha}} x^{a}
$$

with $\tilde{\sigma}_{a}^{\alpha \dot{\alpha}}=\varepsilon^{\alpha \beta} \varepsilon^{\dot{\alpha} \dot{\beta}}\left(\sigma_{a}\right)_{\beta \delta}$, and convert back and forth between the two representations using the relations

$$
\begin{aligned}
& \left(\sigma_{a}\right)_{\alpha \dot{\gamma}}\left(\tilde{\sigma}_{b}\right)^{\beta \dot{\gamma}}+\left(\sigma_{b}\right)_{\alpha \dot{\gamma}}\left(\tilde{\sigma}_{a}\right)^{\beta \dot{\gamma}}=-2 \eta_{a b} \delta_{\alpha}{ }^{\beta}, \\
& \left(\tilde{\sigma}_{a}\right)^{\gamma \dot{\alpha}}\left(\sigma_{b}\right)_{\gamma \dot{\beta}}+\left(\tilde{\sigma}_{b}\right)^{\gamma \dot{\alpha}}\left(\sigma_{a}\right)_{\gamma \dot{\beta}}=-2 \eta_{a b} \delta_{\dot{\beta}}{ }^{\dot{\alpha}},
\end{aligned}
$$

which imply

$$
x^{a}=-\frac{1}{2}\left(\sigma^{a}\right)_{\alpha \dot{\alpha}} x^{\alpha \dot{\alpha}}, \quad \quad x^{a} x_{a}=-\frac{1}{2} x^{\alpha \dot{\alpha}} x_{\alpha \dot{\alpha}} .
$$

A superspace is defined to be a space with coordinates $x^{\alpha \dot{\alpha}}$ of even Graßmann parity Graßmann parity and $\theta^{\alpha}, \bar{\theta}^{\dot{\alpha}}=\left(\theta^{\alpha}\right)^{\dagger}$ of odd Graßmann parity; i.e. anticommuting. The Graßmann parity of a quantity $q$ is symbolised by $\# q$ and capital Latin letters are used to denote collective indices; e.g. the supercoordinates are labelled ${ }^{*} z^{A}=\left(x^{\alpha \dot{\alpha}}, \theta^{\alpha}, \bar{\theta}_{\dot{\alpha}}\right)$ and transform under the $\left(\frac{1}{2}, \frac{1}{2}\right)$, $\left(\frac{1}{2}, 0\right)$ and $\left(0, \frac{1}{2}\right)$ representations, respectively. ${ }^{* *}$ Arbitrary irreducible representations $\left(\frac{m}{2}, \frac{n}{2}\right)$ are given by symmetric tensors

$$
\psi^{\alpha_{1}, \ldots, \alpha_{m}, \dot{\beta}_{1}, \ldots, \dot{\beta}_{n}} \equiv \psi^{\left\{\alpha_{1}, \ldots, \alpha_{m}\right\},\left\{\dot{\beta}_{1}, \ldots, \dot{\beta}_{n}\right\}},
$$

where the weight is chosen such that (anti-)symmetrisation is idempotent, (anti-)

*This convention implies that components of a tensorial object $t_{A_{1} \ldots A_{n}}$ have a varying number of indices. Commas will be used to separate index pairs $\alpha \dot{\alpha}, \beta \dot{\gamma}$ whenever this disambiguation is necessary.

**The latter are (complex) Weyl spinors as opposed to Dirac spinors, which are composed of two Weyl spinors. 


$$
\begin{aligned}
\psi_{\left\{\alpha_{1}, \ldots, \alpha_{N}\right\}} & =\frac{1}{N !} \sum \psi_{\pi\left(\alpha_{1}\right), \ldots, \pi\left(\alpha_{N}\right)}, \\
\psi_{\left[\alpha_{1}, \ldots, \alpha_{N}\right]} & =\frac{1}{N !} \sum \operatorname{sign}(\pi) \psi_{\pi\left(\alpha_{1}\right), \ldots, \pi\left(\alpha_{N}\right)},
\end{aligned}
$$

and (anti-)symmetrisation is performed over only those indices enclosed in braces that are not additionally enclosed in a pair of vertical bars || . From the spin-statistics theorem follows that any physical field $\psi^{\alpha_{1}, \ldots, \alpha_{m}, \dot{\beta}_{1}, \ldots, \dot{\beta}_{n}}$ has Graßmann parity $m+n(\bmod 2)$.

Partial superderivatives $\partial_{A}=\left(\partial_{\alpha \dot{\alpha}}, \partial_{\alpha}, \bar{\partial}^{\dot{\alpha}}\right)$ are defined by

$$
\left[\partial_{A}, z^{B}\right\}=\left(\partial_{A} z^{B}\right):=\delta_{A}^{B}
$$

graded where the $\left(\mathbb{Z}_{2^{-}}\right)$graded commutator is defined by

commutator

$$
[A, B\}:=A B-(-1)^{\# A \# B} B A
$$

Leibniz, Jacobi and obeys the graded Leibniz rule and Jacobi identity

$$
\begin{array}{r}
{[A, B C\}=[A, B\} C+(-1)^{\# A \# B} B[A, C\},} \\
(-1)^{\# A \# C}[A,[B, C\}\}+(\operatorname{cyclic} A \mapsto B \mapsto C)=0 .
\end{array}
$$

The partial derivatives in a flat superspace satisfy

$$
\left[\partial_{A}, \partial_{B}\right\}=0
$$

components

A superfield $f(x, \theta, \bar{\theta})$ on $\mathbb{R}^{4 \mid 4}$ can be defined by a Taylor expansion in the non-commuting coordinates according to

$$
\begin{aligned}
f\left(z^{A}\right)= & A(x)+\theta^{\alpha} \psi_{\alpha}(x)+\bar{\theta}_{\dot{\alpha}} \bar{\psi}^{\dot{\alpha}}(x) \\
& +\theta^{2} F(x)+\bar{\theta}^{2} \bar{F}(x)+\theta \sigma^{a} \bar{\theta} V_{a}(x) \\
& +\bar{\theta}^{2} \theta^{\alpha} \lambda_{\alpha}(x)+\theta^{2} \bar{\theta}_{\dot{\alpha}} \bar{\lambda}^{\dot{\alpha}}(x)+\theta^{2} \bar{\theta}^{2} G(x),
\end{aligned}
$$

where the respective coefficients are called components. Mass dimension and Graßmann parity of the superfield are by definition given by the respective property of the lowest component $A$. This definition of a superfield can be extended to include tensorial fields by simply promoting the components to tensors. 
In a similar manner a superfield can be defined on $\mathbb{C}^{4 \mid 2}$, which is build up from four complex $\left(y^{a}\right)$ and two anticommuting $\left(\theta^{\alpha}\right)$ coordinates. For the remaining part of this introduction, these two superspaces will be referred to as the real $\left(\mathbb{R}^{4 \mid 4}\right)$ and complex $\left(\mathbb{C}^{4 \mid 2}\right)$ superspace respectively. The real superspace is a subspace of the complex superspace, embedded according to

$$
y^{a}=x^{a}+i \theta \sigma^{a} \bar{\theta} .
$$

By this relation holomorphic superfields can be defined on the real super- chiral superfields space (where they are known as chiral superfields) according to

$$
\begin{aligned}
\Phi(x, \theta, \bar{\theta}) & =\Phi\left(x+i \theta \sigma^{a} \bar{\theta}, \theta\right)=\mathrm{e}^{i H} \Phi(x, \theta) \\
H & :=\theta \sigma^{a} \bar{\theta} \partial_{a}
\end{aligned}
$$

where $H$ has been defined with future generalisations in mind. (The current choice of $H$ has the unique property of making super-Poincaré transformations on both spaces coincide, thus providing the only Poincaré invariant embedding of $\mathbb{R}^{4 \mid 4}$ into $\mathbb{C}^{4 \mid 2}$.)

The property $\bar{\partial} \Phi(y)=0$ can be rewritten as

flat covariant

$$
\bar{D}_{\dot{\alpha}} \Phi(x, \theta, \bar{\theta})=0, \quad \bar{D}_{\dot{\alpha}}:=\mathrm{e}^{i H}\left(-\bar{\partial}_{\dot{\alpha}}\right) \mathrm{e}^{-i H}=-\bar{\partial}_{\dot{\alpha}}-i \theta^{\alpha} \partial_{\alpha \dot{\alpha}}
$$

Analogously, for an antichiral field it holds

$$
D_{\alpha} \Phi(x, \theta, \bar{\theta})=0, \quad D_{\alpha}:=\mathrm{e}^{-i H}\left(\partial_{\alpha}\right) \mathrm{e}^{i H}=\partial_{\alpha}+i \theta^{\alpha} \partial_{\alpha \dot{\alpha}}
$$

The set of derivatives $D_{A}=\left(\partial_{a}, D_{\alpha}, \bar{D}^{\dot{\alpha}}\right)$ has the property of commuting with the supersymmetry generators and mapping a tensor superfield into a tensor superfield with respect to the Lorentz group. Hence, they are called (flat) covariant derivatives. The observant reader has noticed the unusual sign in front of $\bar{\partial}_{\dot{\alpha}}$ in definition (6.22), which is related to convenient complex conjugation properties as will be explained below. While partial derivatives obey trivial (anti-)commutation rules, this is no longer true for covariant derivatives $\left(\left\{D_{\alpha}, \bar{D}_{\dot{\alpha}}\right\}=-2 i \partial_{\alpha \dot{\alpha}}\right)$, and consequently special attention has to be paid to the reordering upon complex conjugation, in particular Hermitean and complex conjugation no longer coincide. 


\begin{tabular}{c|c|c|c}
\multicolumn{4}{c}{ ConjugATIONS } \\
\hline $\mathcal{O}$ & $\mathcal{O}^{\dagger}$ & $\mathcal{O}^{*}$ & $\mathcal{O}^{\mathrm{T}}$ \\
\hline $\mathcal{O}_{1} \ldots \mathcal{O}_{n}$ & $\mathcal{O}_{n}^{\dagger} \ldots \mathcal{O}_{1}^{\dagger}$ & $\pi_{\# F} \mathcal{O}_{1}^{*} \ldots \mathcal{O}_{n}^{*}$ & $\pi_{\# F} \mathcal{O}_{n}^{\mathrm{T}} \ldots \mathcal{O}_{1}^{\mathrm{T}}$ \\
\hline$\psi^{\alpha}$ & $\bar{\psi}^{\dot{\alpha}}$ & $\bar{\psi}^{\dot{\alpha}}$ & $\psi^{\alpha}$ \\
\hline$\psi^{\alpha_{1} \ldots \alpha_{m} \dot{\beta}_{1} \ldots \dot{\beta}_{n}}$ & $\bar{\psi}^{\dot{\beta}_{n} \ldots \dot{\beta}_{1} \alpha_{m} \ldots \alpha_{1}}$ & $\pi_{n} \pi_{m} \bar{\psi}^{\dot{\beta}_{n} \ldots \dot{\beta}_{1} \alpha_{m} \ldots \alpha_{1}}$ & $\pi_{n} \pi_{m} \psi^{\alpha_{m} \ldots \alpha_{1} \dot{\beta}_{n} \ldots \dot{\beta}_{1}}$ \\
\hline$\partial_{a}$ & $-\partial_{a}$ & $\partial_{a}$ & $-\partial_{a}$ \\
\hline$\partial_{\alpha}$ & $\bar{\partial}_{\dot{\alpha}}$ & $-\bar{\partial}_{\dot{\alpha}}$ & $-\partial_{\alpha}$ \\
\hline$D_{a}$ & $-D_{a}$ & $D_{a}$ & $-D_{a}$ \\
\hline$D_{\alpha}$ & $-\bar{D}_{\dot{\alpha}}$ & $\bar{D}_{\dot{\alpha}}$ & $-D_{\alpha}$ \\
\hline
\end{tabular}

Table 6.1: Definition of the Hermitean and complex conjugate as well as transposition (from left to right). The symbol

$$
\pi_{m}:=(-1)^{\left\lfloor\frac{m}{2}\right\rfloor}=(-1)^{\frac{1}{2} m(m-1)}
$$

denotes the sign change induced by reversing the order of $m$ anticommuting objects while $\# F$ is the number of fermionic terms in the corresponding expression.

The Hermitean conjugate $\mathcal{O}^{\dagger}$ and transpose $\mathcal{O}^{\mathrm{T}}$ of an operator $\mathcal{O}$ are respectively defined by

$$
\begin{aligned}
\int \overline{\mathcal{O}^{\dagger} \chi} \psi & :=\int \bar{\chi} \mathcal{O} \psi, \\
\int\left(\mathcal{O}^{\mathrm{T}} \chi\right) \psi & :=(-1)^{\# \chi \# \mathcal{O}} \int \chi \mathcal{O} \psi,
\end{aligned}
$$

which additionally allows to define the complex conjugate by

$$
\mathcal{O}^{*}:=\left(\mathcal{O}^{\dagger}\right)^{\mathrm{T}}
$$

In particular, these definitions imply the following reorderings

$$
\begin{aligned}
\left(\mathcal{O}_{1} \ldots \mathcal{O}_{N}\right)^{\dagger} & =\mathcal{O}_{N}^{\dagger} \ldots \mathcal{O}_{1}^{\dagger}, \\
\left(\mathcal{O}_{1} \ldots \mathcal{O}_{N}\right)^{\mathrm{T}} & =(-1)^{\# \mathcal{O}_{1} \# \mathcal{O}_{2}} \mathcal{O}_{N}^{\mathrm{T}} \ldots \mathcal{O}_{1}^{\mathrm{T}}, \\
\left(\mathcal{O}_{1} \ldots \mathcal{O}_{N}\right)^{*} & =(-1)^{\# \mathcal{O}_{1} \# \mathcal{O}_{2}} \mathcal{O}_{1}^{*} \ldots \mathcal{O}_{N}^{*}
\end{aligned}
$$

From 


$$
\begin{gathered}
\left\{\left(\bar{\partial}_{\dot{\alpha}}\right)^{\dagger},\left(\bar{z}^{\dot{\beta}}\right)^{\dagger}\right\}=\left\{\bar{\partial}_{\dot{\alpha}}, \bar{z}^{\dot{\beta}}\right\}^{\dagger}=\left(\delta_{\dot{\alpha}}^{\dot{\beta}}\right)^{\dagger}=\delta_{\alpha}{ }^{\beta}=\left\{\partial_{\alpha}, z^{\beta}\right\} \\
-\left[\left(\partial_{a}\right)^{\dagger},\left(z^{a}\right)^{\dagger}\right]=\left[\partial_{a}, z^{b}\right]^{\dagger}=\left(\delta_{a}{ }^{b}\right)^{\dagger}=\delta_{a}{ }^{b}=\left[\partial_{a}, z^{b}\right]
\end{gathered}
$$

one may deduce

$$
\begin{aligned}
& \left(\partial_{a}\right)^{\dagger}=-\partial_{a} \\
& \left(\partial_{\alpha}\right)^{\dagger}=\bar{\partial}_{\dot{\alpha}}
\end{aligned}
$$

while the transpose $\partial_{A}^{T}=-\partial_{A}$ is determined by partial integration. So complex conjugation of a spinor partial derivative involves an additional minus sign compared to other fermionic objects. As complex conjugation is an operation which will be employed quite frequently when working directly with the supergravity algebra, the definition of covariant spinor derivatives (6.22) involves an additional minus sign for compensation. The conjugation rules are summarised in Table 6.1. As one can see, for the case of (anti-)commuting objects - "numbers" - Hermitean conjugation and complex conjugation are the same.

In the supergravity literature, the use of different notations and con- conventional ventions is quite common. In particular it crucially depends on the task traps to be performed, which conventions are the most suitable. This thesis follows closely the conventions of [99], which contain the potential trap that for an antisymmetric tensor

$$
\psi_{\alpha \beta} \sim \varepsilon_{\alpha \beta}
$$

the corresponding contragradient tensor reads

$$
\psi^{\alpha \beta}=\varepsilon^{\alpha \gamma} \varepsilon^{\beta \delta} \psi_{\gamma \delta} \sim \varepsilon^{\alpha \gamma} \varepsilon^{\beta \delta} \varepsilon_{\gamma \delta}=-\varepsilon^{\alpha \beta}
$$

as a consequence of the conventions used for raising and lowering operators.

The other major source of this compilation [98] uses an imaginary symplectic metric, which introduce a relative minus sign for complex conjugation of contragradient indices. Additionally, there appears a minus sign in the complex conjugation of spinorial covariant superderivatives $D_{\alpha}=\left(\bar{D}_{\dot{\alpha}}\right)^{\dagger}=-\left(\bar{D}_{\dot{\alpha}}\right)^{*}$. Furthermore, quadratic quantities $D^{2}$ contain a 


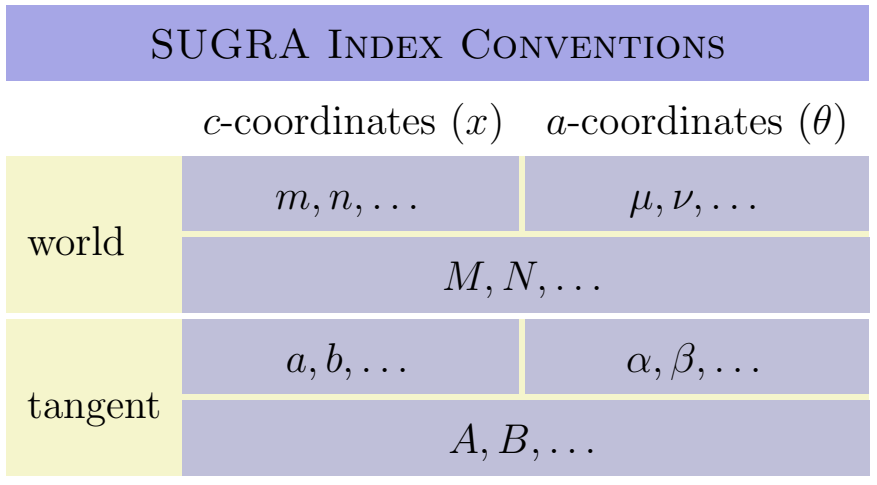

Table 6.2: Superfield Supergravity Index Conventions

factor of one half, which materialises upon partial integration.

\subsection{Superspace Supergravity}

In analogy to the non-supersymmetric case, a pseudo-Riemannian supermanifold is defined by an atlas of maps from open sets of points on the supermanifold to coordinates in flat superspace. When there is curvature, in general more than one map is required to cover the whole manifold and the maps are distorted in the sense, that a non-Minkowski metric is needed to capture this distortion in terms of those superspace coordinates, which shall be called world or curved coordinates coordinates $z^{M}=\left(z^{m}, \theta^{\mu}, \bar{\theta}_{\dot{\mu}}\right)$. To each point of the supermanifold one may attach a tangent superspace (also referred to as flat), whose coordinates are called $z^{A}=\left(z^{a}, \theta^{\alpha}, \bar{\theta}_{\dot{\alpha}}\right)$. The distinction of flat vs. curved will also be made in referring to the indices only as indicated in Table 6.2. superspace general coordinate transformations, realised as gauged curved superspace translations, are augmented by an additional set of superlocal Lorentz transformations acting on the tangent space only. The reason is that without this doubling spinors can only be realised non-linearly, which is inconvenient [98, p. 235].

A first order differential operator, expressed as

$$
K=K^{M} \partial_{M}+\frac{1}{2} K^{a b} M_{a b}=K^{M} \partial_{M}+K^{\alpha \beta} M_{\alpha \beta}+K^{\dot{\alpha} \dot{\beta}} \bar{M}_{\dot{\alpha} \dot{\beta}},
$$


therefore allows to define covariant transformation under combined supercoordinate and super-Lorentz transformations according to

$$
X \mapsto \mathrm{e}^{K} X \mathrm{e}^{-K}
$$

The $\mathfrak{s l}(2, \mathbb{C})$ versions $M_{\alpha \beta}=\frac{1}{2}\left(\sigma^{a b}\right)_{\alpha \beta} M_{a b}$ and $\bar{M}_{\dot{\alpha} \dot{\beta}}=\frac{1}{2}\left(\tilde{\sigma}^{a b}\right)_{\dot{\alpha} \dot{\beta}} M_{a b}$ of Lorenz generators the Lorentz generator $M_{a b}$ act on the corresponding indices (i.e. only on indices of the same kind) according to

$$
\begin{aligned}
& M_{\beta \gamma} \psi_{\alpha_{1} \ldots \alpha_{n}}=\frac{1}{2} \sum_{i}\left(\varepsilon_{\alpha_{i} \beta} \psi_{\gamma \alpha_{1} \ldots \phi_{i} \ldots \alpha_{n}}+\varepsilon_{\alpha_{i} \gamma} \psi_{\beta \alpha_{1} \ldots \alpha_{i} \ldots \alpha_{n}}\right), \\
& \bar{M}_{\dot{\beta} \dot{\gamma}} \psi_{\dot{\alpha}_{1} \ldots \dot{\alpha}_{n}}=\frac{1}{2} \sum_{i}\left(\varepsilon_{\dot{\alpha}_{i} \dot{\beta}} \psi_{\dot{\gamma} \dot{\gamma}_{1} \ldots \dot{\alpha}_{i} \ldots \dot{\alpha}_{n}}+\varepsilon_{\dot{\alpha}_{i} \dot{\gamma}} \psi_{\dot{\beta} \dot{\alpha}_{1} \ldots \dot{\alpha}_{i} \ldots \dot{\alpha}_{n}}\right) .
\end{aligned}
$$

In particular, it holds

$$
\begin{aligned}
M_{\beta \gamma} \psi_{\alpha} & =\frac{1}{2}\left(\varepsilon_{\alpha \beta} \psi_{\gamma}+\varepsilon_{\alpha \gamma} \psi_{\gamma}\right), \\
M_{\beta \gamma} \psi^{\alpha} & =\frac{1}{2}\left(\delta_{\beta}^{\alpha} \psi_{\gamma}+\delta_{\gamma}^{\alpha} \psi_{\beta}\right), \\
M_{\alpha \beta} \psi^{\beta} & =\frac{3}{2} \psi_{\beta} .
\end{aligned}
$$

In analogy to ordinary gravity (with torsion) one may define a derivative

curved covariant derivatives

$$
\mathcal{D}_{A}=E_{A}+\Omega_{A}
$$

that transforms covariantly under (6.36) by adding a vierbein field $E_{A}:=$ $E_{A}{ }^{M} \partial_{M}$ and a superconnection

$$
\Omega_{A}:=\frac{1}{2} \Omega_{A}{ }^{B C} M_{B C}=\Omega_{A}{ }^{\beta \gamma} M_{\beta \gamma}+\Omega_{A}{ }^{\dot{\beta} \dot{\gamma}} \bar{M}_{\dot{\beta} \dot{\gamma}}
$$

The vierbein obeys the algebra

anholonomy

$$
\begin{aligned}
{\left[E_{A}, E_{B}\right\} } & =C_{A B}{ }^{C} E_{C}, \\
C_{A B}{ }^{C} & =\left(E_{A} E_{B}^{M}-(-1)^{\# A \# B} E_{B} E_{A}{ }^{M}\right) E_{M}^{C},
\end{aligned}
$$

where $C_{A B}{ }^{C}$ are the supersymmetric generalisation of anholonomy coefficients. The non-degenerate supermatrix $E_{A}{ }^{M}$ can be used to convert 
between world and tangent indices according to

$$
V_{A}=E_{A}^{M} V_{M}
$$

and the bosonic submatrix $E_{a}{ }^{m}$ is the well known vierbein field of gravity obeying

$$
\eta_{a b}=g_{m n} E_{a}{ }^{m} E_{b}{ }^{n}
$$

curvature, torsion

The covariant derivatives form an algebra

$$
\begin{aligned}
{\left[\mathcal{D}_{A}, \mathcal{D}_{B}\right\} } & =T_{A B}+R_{A B}, \\
T_{A B} & :=T_{A B}{ }^{C} \partial_{C}, \\
R_{A B} & :=\frac{1}{2} R_{A B}{ }^{b c} M_{b c}=R_{A B}{ }^{\beta \gamma} M_{\beta \gamma}+R_{A B}{ }^{\dot{\beta} \dot{\gamma}} \bar{M}_{\dot{\beta} \dot{\gamma}},
\end{aligned}
$$

with $T_{A B}=-(-1)^{\# A \# B} T_{B A}$ the supertorsion and $R_{A B}=$ $-(-1)^{\# A \# B} R_{B A}$ the supercurvature, which may be completely expressed in terms of the supertorsion as a consequence of the Bianchi identities. The latter are just the Jacobi identities (6.17) for the algebra (6.45).

\subsection{Non-minimal Supergravity}

The algebra above is a highly reducible representation of supergravity. To extract the physical degrees of freedom a number of constraints has to be

conventional constraints

$$
\left.\begin{array}{l}
T_{\alpha \dot{\beta}}^{\gamma}=T_{\alpha \dot{\beta}}^{\dot{\gamma}}=R_{\alpha \beta}{ }^{c d}=0, \\
T_{\alpha \dot{\beta}}{ }^{c}=-2 i \sigma_{\alpha \dot{\beta}}^{c}
\end{array}\right\} \Longleftrightarrow \mathcal{D}_{\alpha \dot{\alpha}}=-2 i\left\{\mathcal{D}_{\alpha}, \overline{\mathcal{D}}_{\dot{\alpha}}\right\},
$$

which are equivalent to redefinitions of the algebra's constituents, and representation preserving constraints representation preserving constraints

$$
T_{\alpha \beta}^{c}=T_{\dot{\alpha} \dot{\beta}}^{c}=T_{\alpha \beta} \dot{\gamma}^{\prime}=T_{\dot{\alpha} \dot{\beta}}^{\gamma}=0,
$$


which imply the existence of (anti-)chiral superfields by ensuring the WessZumino consistency condition

$$
\overline{\mathcal{D}}_{\dot{\alpha}} \chi=0 \Longrightarrow\left\{\overline{\mathcal{D}}_{\dot{\alpha}}, \overline{\mathcal{D}}_{\dot{\beta}}\right\} \chi=0
$$

While the Bianchi identities are trivially fulfilled by the unconstrained derivatives, this is no longer true, when introducing constraints whose solving of Bianchi consequences for the remaining torsion fields have to be evaluated. Since this procedure is straight-forward, it will not be reproduced here due to the length of the calculation and the fact, that it may be found in the literature [98-101] under the name of "solving the Bianchi identities".

After solving the Bianchi identities, all torsions and curvatures can be expressed in terms of a few basic fields,

$$
\begin{aligned}
T_{\alpha} & :=(-1)^{\# B} T_{\alpha B}{ }^{B}, \\
G_{\alpha \dot{\alpha}} & :=i T^{\beta,}{ }_{\beta \dot{\alpha}, \alpha}+i T^{\dot{\beta},}{ }_{\alpha \dot{\beta}, \dot{\alpha}}, \\
R & :=\frac{1}{12} R^{\dot{\alpha} \dot{\beta}} \dot{\alpha} \dot{\beta}, \\
W_{\alpha \beta \gamma} & :=\frac{1}{2} T_{\{i a}{ }^{\dot{\beta},}{ }_{\beta|\dot{\beta}|, \gamma\}},
\end{aligned}
$$

where $R$ and $\bar{R}$ are chiral and antichiral superfields, $G_{\alpha \dot{\alpha}}$ is real, and $T_{\alpha}$, $W_{\alpha \beta \gamma}$ are complex superfields, all of which are subject to a set of Bianchi identities and obey the so-called "non-minimal supergravity algebra".

\subsubsection{Algebra and Bianchi identities}

The non-minimal supergravity algebra is defined by the following three defining relations (anti-)commutators,

$$
\begin{aligned}
&\left\{\mathcal{D}_{\alpha}, \overline{\mathcal{D}}_{\dot{\alpha}}\right\}=-2 i \mathcal{D}_{\alpha \dot{\alpha}}, \\
&\left\{\mathcal{D}_{\alpha}, \mathcal{D}_{\beta}\right\}=-4 \bar{R} M_{\alpha \beta}, \\
& {\left[\overline{\mathcal{D}}_{\dot{\alpha}}, \mathcal{D}_{\beta \dot{\beta}}\right]=\varepsilon_{\dot{\alpha} \dot{\beta}}\left[\frac{1}{2} \bar{T}^{\dot{\gamma}} \mathcal{D}_{\beta \dot{\gamma}}-i\left(R+\frac{1}{8} \overline{\mathcal{D}}_{\dot{\gamma}} \bar{T}^{\dot{\gamma}}-\frac{1}{16} \bar{T}^{2}\right) \mathcal{D}_{\beta}\right.} \\
& \quad-i \bar{\psi}_{\beta}{ }^{\dot{\gamma}} \overline{\mathcal{D}}_{\dot{\gamma}}+i\left(\overline{\mathcal{D}}^{\dot{\delta}}-\frac{1}{2} \bar{T}^{\dot{\delta}}\right) \bar{\psi}_{\beta}{ }^{\dot{\gamma}} \bar{M}_{\dot{\delta} \dot{\gamma}} \\
&\left.\quad+2 i X^{\gamma} M_{\beta \gamma}-2 i W_{\beta}^{\gamma \delta} M_{\gamma \delta}\right]-i\left(\mathcal{D}_{\beta} R\right) \bar{M}_{\dot{\alpha} \dot{\beta}} .
\end{aligned}
$$


implications The missing relations can be determined from the Bianchi identities (see below) and complex conjugation.

$$
\begin{aligned}
&\left\{\overline{\mathcal{D}}_{\dot{\alpha}}, \overline{\mathcal{D}}_{\dot{\beta}}\right\}=4 R \bar{M}_{\dot{\alpha} \dot{\beta}}, \\
& {\left[\mathcal{D}_{\alpha}, \mathcal{D}_{\beta \dot{\beta}}\right]=\varepsilon_{\alpha \beta}\left[\frac{1}{2} T^{\gamma} \mathcal{D}_{\gamma \dot{\beta}}+i\left(\bar{R}+\frac{1}{8} \mathcal{D}^{\gamma} T_{\gamma}-\frac{1}{16} T^{2}\right) \overline{\mathcal{D}}_{\dot{\beta}}\right.} \\
&+i \psi_{\dot{\beta}}^{\gamma} \mathcal{D}_{\gamma}+i\left(\mathcal{D}^{\delta}-\frac{1}{2} T^{\delta}\right) \psi_{\dot{\beta}}^{\gamma} M_{\delta \gamma} \\
&\left.\quad-2 i \bar{X}^{\dot{\gamma}} \bar{M}_{\dot{\beta} \dot{\gamma}}+2 i \bar{W}_{\dot{\beta}}^{\dot{\gamma} \dot{\delta}} M_{\dot{\gamma} \dot{\delta}}\right]+i\left(\overline{\mathcal{D}}_{\dot{\beta}} \bar{R}\right) M_{\alpha \beta}, \\
& {\left[\mathcal{D}_{\alpha \dot{\alpha}}, \mathcal{D}_{\beta \dot{\beta}}\right]=\frac{i}{2}\left\{\left[\mathcal{D}_{\alpha}, \mathcal{D}_{\beta \dot{\beta}}\right], \overline{\mathcal{D}}_{\dot{\alpha}}\right\}+\frac{i}{2}\left\{\left[\overline{\mathcal{D}}_{\dot{\alpha}}, \mathcal{D}_{\beta \dot{\beta}}\right], \mathcal{D}_{\alpha}\right\}, }
\end{aligned}
$$

with the abbreviations

$$
\begin{aligned}
\psi_{\alpha \dot{\alpha}}= & G_{\alpha \dot{\alpha}}-\frac{1}{8} \mathcal{D}_{\alpha} \bar{T}_{\dot{\alpha}}-\frac{1}{8} \overline{\mathcal{D}}_{\dot{\alpha}} T_{\alpha}, \\
X_{\alpha}= & \frac{1}{12}\left[\left(\overline{\mathcal{D}}_{\dot{\gamma}}-\frac{1}{2} \bar{T}_{\dot{\gamma}}\right)\left(\overline{\mathcal{D}}^{\dot{\gamma}}-\frac{1}{2} \bar{T}^{\dot{\gamma}}\right)-4 R\right] T_{\alpha} \\
& +\frac{1}{12}\left[2 \psi_{\alpha \dot{\alpha}}+\left(\overline{\mathcal{D}}_{\dot{\alpha}}-\frac{1}{2} \bar{T}_{\dot{\alpha}}\right)\left(\mathcal{D}_{\alpha}-\frac{1}{2} T_{\alpha}\right)\right. \\
& \left.+\frac{1}{2}\left(\mathcal{D}_{\alpha}-T_{\alpha}\right)\left(\overline{\mathcal{D}}_{\dot{\alpha}}-\frac{1}{2} \bar{T}_{\dot{\alpha}}\right)\right] \bar{T}^{\dot{\alpha}} .
\end{aligned}
$$

Bianchi identities

The Bianchi identities expressed in terms of the supertorsion components read

$$
\begin{aligned}
\overline{\mathcal{D}}_{\dot{\alpha}} R=0, \quad G_{a} & =\bar{G}_{a}, \quad W_{\alpha \beta \gamma}=W_{\{\alpha \beta \gamma\}}, \\
\mathcal{D}_{\alpha} T_{\beta}+\mathcal{D}_{\beta} T_{\alpha} & =0, \\
\left(\overline{\mathcal{D}}^{\dot{\alpha}}-\frac{1}{2} \bar{T}^{\dot{\alpha}}\right) \psi_{\alpha \dot{\alpha}} & =\mathcal{D}_{\alpha} R, \quad\left(\overline{\mathcal{D}}_{\dot{\alpha}}-\frac{1}{2} \bar{T}_{\dot{\alpha}}\right) W_{\alpha \beta \gamma}=0, \\
\left(\mathcal{D}^{\gamma}-T^{\gamma}\right) W_{\alpha \beta \gamma} & =\frac{i}{2}\left(\mathcal{D}_{\alpha}{ }^{\dot{\alpha}}-\frac{i}{2}\left(\mathcal{D}_{\alpha} \bar{T}^{\dot{\alpha}}\right)\right) \psi_{\beta \dot{\alpha}}+(\alpha \leftrightarrow \beta) .
\end{aligned}
$$

\subsubsection{Partial Integration}

From the supergravity algebra (6.45) it can be shown that

$$
(-1)^{\# A} E^{-1} \mathcal{D}_{A} V^{A}-(-1)^{\# B} V^{A} T_{A B}^{B}=\left(E^{-1} V^{A}\right) \overleftarrow{E}_{A}
$$


which implies

$$
\begin{aligned}
\int d^{8} z E^{-1}\left(\mathcal{D}_{\alpha \dot{\alpha}}-(-1)^{\# B} T_{a B}{ }^{B}\right) V^{\alpha \dot{\alpha}} & =0, \\
\int d^{8} z E^{-1}\left(\mathcal{D}_{\alpha}+T_{\alpha}\right) V^{\alpha} & =0, \\
\int d^{8} z E^{-1}\left(\overline{\mathcal{D}}_{\dot{\alpha}}+T_{\dot{\alpha}}\right) V^{\dot{\alpha}} & =0 .
\end{aligned}
$$

$E^{-1}:=\operatorname{sdet}^{-1} E_{A}{ }^{M}$ is the real superspace analogue of $\sqrt{-g_{m n}}$.

Clearly it is a natural alternative to consider the combination $\mathcal{D}_{\alpha}+T_{\alpha}$ as the basic covariant derivative. Then $T_{\alpha}$ takes over the rôle of a $\mathrm{U}(1)_{R}$ connection, an approach chosen in [98].

\subsubsection{Superdeterminant}

In the last Section the superdeterminant has been introduced and its definition shall follow here, belatedly. In analogy to the non-supersymmetric case the superdeterminant is the exponential of the logarithm's supertrace

$$
\operatorname{sdet} A^{M}{ }_{N}:=\exp \mathrm{S} \operatorname{Tr} \ln A^{M}{ }_{N}
$$

where the supertrace is

supertrace

$$
\mathrm{S} \operatorname{Tr} A^{M}{ }_{N}:=(-1)^{\# M} A^{M}{ }_{M},
$$

which is cyclic and invariant under a suitably defined supertransposition $\left(A^{\mathrm{sT}}\right)_{M}{ }^{N}:=(-1)^{\# N+\# M \# N} A^{N}{ }_{M}$.

For practical calculations, the following theorem is much more important

$$
\begin{aligned}
z^{M} & =\mathrm{e}^{-K} z^{M}, & & K=K^{M} \partial_{M}, \\
\operatorname{sdet} \frac{\partial z^{M}}{\partial z^{N}} & =\left(1 \cdot \mathrm{e}^{\overleftarrow{K}}\right), & \overleftarrow{K} & =K^{M} \overleftarrow{\partial}_{M} .
\end{aligned}
$$

The right partial derivative $\overleftarrow{\partial}_{M}$ in $\overleftarrow{K}$ acts on the components $K^{M}$ and right operator 
everything to the left of $\overleftarrow{K}$, such that

$$
\overleftarrow{K}=(-1)^{\# M} \overleftarrow{\partial}_{M} K^{M}+(-1)^{\# M}\left(\partial_{M} K^{M}\right)
$$

Additionally the following rule holds

$$
\left(1 \cdot \mathrm{e}^{\overleftarrow{K}}\right)\left(\mathrm{e}^{K} \Phi\right)=\left(\Phi \cdot \mathrm{e}^{\overleftarrow{K}}\right)
$$

Proofs for any of these statements can be found in the literature, in particular [99].

\subsubsection{Super-Weyl Transformations}

While the algebra of the previous Sections is by construction invariant under general supercoordinate and superlocal Lorentz transformations, it is in addition invariant under transformations of the vierbein of the form

$$
\begin{aligned}
E_{\alpha} & \mapsto L E_{\alpha}, \\
\bar{E}_{\dot{\alpha}} & \mapsto \bar{L} \bar{E}_{\dot{\alpha}}, \\
E_{\alpha \dot{\alpha}} & \mapsto L \bar{L} E_{\alpha \dot{\alpha}}, \\
E & \mapsto(L \bar{L})^{2} E,
\end{aligned}
$$

which are easily seen to represent Weyl transformation of the bosonic vierbein component, when restricting $L$ to (the real part of) its lowest component. The unconstrained complex superfield $L=\exp \left(\frac{1}{2} \Delta+\frac{i}{2} \kappa\right)$ parametrises mixed superlocal scale transformations (by $\Delta$ ) and superlocal chiral transformations (by $\kappa$ ). The latter can also be understood as local $\mathrm{U}(1)_{R}$ transformations.

The elements of the non-minimal supergravity algebra transform under this symmetry as

$$
\begin{aligned}
& \mathcal{D}_{\alpha} \mapsto L \mathcal{D}_{\alpha}-2\left(\mathcal{D}^{\beta} L\right) M_{\alpha \beta}, \\
& \overline{\mathcal{D}}_{\dot{\alpha}} \mapsto \bar{L} \overline{\mathcal{D}}_{\dot{\alpha}}-2\left(\overline{\mathcal{D}}^{\dot{\beta}} \bar{L}\right) \bar{M}_{\dot{\alpha} \dot{\beta}}, \\
& T_{\alpha} \mapsto L T_{\alpha}+\mathcal{D}_{\alpha}^{\prime} \ln \left(L^{4} \bar{L}^{2}\right), \\
& R \mapsto-\frac{1}{4}\left(\overline{\mathcal{D}}^{2}-4 R\right) \bar{L}^{2}
\end{aligned}
$$




\subsubsection{Prepotentials}

As a consequence of Frobenius' theorem, the vierbein field, which is subject to the constraint (6.41) can be decomposed into unconstrained superfields $F, W$ and $N_{\alpha}{ }^{\mu}$, called prepotentials,

$$
\begin{aligned}
& E_{\alpha}=F N_{\alpha}^{\mu} \mathrm{e}^{W} \partial_{\mu} \mathrm{e}^{-W}, \quad \operatorname{det} N_{\alpha}^{\mu}=1, \\
& \bar{E}_{\dot{\alpha}}=-\bar{F} \bar{N}_{\dot{\alpha}}{ }^{\dot{\mu}} \mathrm{e}^{\bar{W}} \bar{\partial}_{\dot{\mu}} \mathrm{e}^{-\bar{W}} .
\end{aligned}
$$

Because the "superscale" field $F$ has been introduced to allow the choice $\operatorname{det} N_{\alpha}{ }^{\mu}=1$, it is also the only prepotential that transforms under superWeyl transformations: $F \mapsto L F$. Under coordinate transformations induced by $K=K^{M} \partial_{M}=\bar{K}$, all prepotentials transform covariantly,

$$
F^{\prime}=\left(\mathrm{e}^{K} F\right), \quad\left(N_{\alpha}{ }^{\mu}\right)^{\prime}=\left(\mathrm{e}^{K} N_{\alpha}{ }^{\mu}\right), \quad W^{\prime}=\left(\mathrm{e}^{K} W\right),
$$

while only $N_{\alpha}{ }^{\mu}$ transforms under superlocal transformations

$$
\left(N_{\alpha}{ }^{\mu}\right)^{\prime}=\left(\mathrm{e}^{\frac{1}{2} K^{a b} M_{a b}}\right) N_{\alpha}{ }^{\mu} .
$$

While all supergravity superfields can be expressed in terms of prepotentials, only the two simple expressions

$$
\begin{aligned}
T_{\alpha} & =E_{\alpha} \ln \left[E F^{2}\left(1 \cdot \mathrm{e}^{\overleftarrow{W}}\right)\right], \\
R & =-\frac{1}{4} \hat{\bar{E}}_{\dot{\mu}} \hat{\bar{E}}^{\dot{\mu}} \bar{F}^{2}
\end{aligned}
$$

shall be given here with the semi-covariant vierbein $\hat{E}$ defined by

semi-covariant vierbein

$$
\begin{array}{rlr}
\hat{E}_{\alpha} & :=F^{-1} E_{\alpha}, \quad \hat{E}_{\alpha}=: N_{\alpha}{ }^{\mu} \hat{E}_{\mu} \\
\hat{\bar{E}}_{\dot{\alpha}} & :=\bar{F}^{-1} \bar{E}_{\dot{\alpha}}, \\
\hat{E}_{\alpha \dot{\alpha}} & :=\frac{i}{2}\left\{\hat{E}_{\alpha}, \hat{\bar{E}}_{\dot{\alpha}}\right\} .
\end{array}
$$

There is an additional prepotential $\varphi$, the chiral compensator, that can be chosen to take over the rôle of $F$, see Section refsec:superweyltrafos. 


\subsection{Minimal Supergravity}

From the non-minimal supergravity algebra, a formulation containing less auxiliary fields may be obtained by setting $T_{\alpha}=0$. This has a number of consequences: The algebra simplifies considerably, $W_{\alpha \beta \gamma}$ becomes a chiral field and super-Weyl transformations can be formulated using a chiral parameter field.

\subsubsection{Algebra and Bianchi Identities}

The minimal supergravity algebra is determined by the three (anti-) commutators $\left\{\mathcal{D}_{\alpha}, \overline{\mathcal{D}}_{\dot{\alpha}}\right\},\left\{\mathcal{D}_{\alpha}, \mathcal{D}_{\beta}\right\},\left[\mathcal{D}_{\alpha}, \overline{\mathcal{D}}_{\beta \dot{\beta}}\right]$, which are listed below with some of their straight-forward implications

$$
\begin{aligned}
& \left\{\mathcal{D}_{\alpha}, \overline{\mathcal{D}}_{\dot{\alpha}}\right\}=-2 i \mathcal{D}_{\alpha \dot{\alpha}}, \\
& \left\{\mathcal{D}_{\alpha}, \mathcal{D}_{\beta}\right\}=-4 \bar{R} M_{\alpha \beta} \text {, } \\
& \left\{\overline{\mathcal{D}}_{\dot{\alpha}}, \overline{\mathcal{D}}_{\dot{\beta}}\right\}=4 R \bar{M}_{\dot{\alpha} \dot{\beta}} \text {, } \\
& \mathcal{D}_{\alpha} \mathcal{D}_{\beta}=\frac{1}{2} \varepsilon_{\alpha \beta} \mathcal{D}^{2}-2 \bar{R} M_{\alpha \beta}, \\
& \overline{\mathcal{D}}_{\dot{\alpha}} \overline{\mathcal{D}}_{\dot{\beta}}=-\frac{1}{2} \varepsilon_{\dot{\alpha} \dot{\beta}} \overline{\mathcal{D}}^{2}+2 R \bar{M}_{\dot{\alpha} \dot{\beta}}, \\
& \mathcal{D}_{\alpha} \mathcal{D}^{2}=4 \bar{R} \mathcal{D}^{\beta}\left(\varepsilon_{\alpha \beta}+M_{\alpha \beta}\right), \\
& \mathcal{D}^{2} \mathcal{D}_{\alpha}=-2 \bar{R} \mathcal{D}^{\beta}\left(\varepsilon_{\alpha \beta}+M_{\alpha \beta}\right), \\
& {\left[\mathcal{D}^{2}, \overline{\mathcal{D}}_{\dot{\alpha}}\right]=-4\left(G_{\alpha \dot{\alpha}}+i \mathcal{D}_{\alpha \dot{\alpha}}\right) \mathcal{D}^{\alpha}+4 \bar{R} \overline{\mathcal{D}}_{\dot{\alpha}}} \\
& -4\left(\mathcal{D}^{\gamma} G_{\dot{\alpha}}^{\delta}\right) M_{\gamma \delta}+8 \bar{W}_{\dot{\alpha}}^{\dot{\gamma} \dot{\delta}} \bar{M}_{\dot{\gamma} \dot{\delta}}, \\
& {\left[\overline{\mathcal{D}}^{2}, \mathcal{D}_{\alpha}\right]=2 i\left[\overline{\mathcal{D}}^{\dot{\alpha}}, \mathcal{D}_{\alpha \dot{\alpha}}\right]+4 i \mathcal{D}_{\alpha \dot{\alpha}} \overline{\mathcal{D}}^{\dot{\alpha}}} \\
& =-4\left(G_{\alpha \dot{\alpha}}-i \mathcal{D}_{\alpha \dot{\alpha}}\right) \overline{\mathcal{D}}^{\dot{\alpha}}+4 R \mathcal{D}_{\alpha}-4\left(\overline{\mathcal{D}}^{\dot{\gamma}} G_{\alpha} \dot{\delta}\right) \bar{M}_{\dot{\gamma} \dot{\delta}}+8 W_{\alpha}{ }^{\gamma \delta} M_{\gamma \delta}, \\
& {\left[\overline{\mathcal{D}}_{\dot{\alpha}}, \mathcal{D}_{\beta \dot{\beta}}\right]=-i \varepsilon_{\dot{\alpha} \dot{\beta}}\left(R \mathcal{D}_{\beta}+G_{\beta} \dot{\dot{\gamma}} \overline{\mathcal{D}}_{\dot{\gamma}}\right)} \\
& -i\left(\mathcal{D}_{\beta} R\right) \bar{M}_{\dot{\alpha} \dot{\beta}}+i \varepsilon_{\dot{\alpha} \dot{\beta}}\left(\overline{\mathcal{D}}^{\dot{\gamma}} G_{\beta}{ }^{\dot{\delta}}\right) \bar{M}_{\dot{\gamma} \dot{\delta}}-2 i \varepsilon_{\dot{\alpha} \dot{\beta}} W_{\beta}^{\gamma \delta} M_{\gamma \delta}, \\
& {\left[\overline{\mathcal{D}}^{\dot{\beta}}, \mathcal{D}_{\beta \dot{\beta}}\right]=-2 i\left(R \mathcal{D}_{\beta}+G_{\beta}{ }^{\dot{\gamma}} \overline{\mathcal{D}}_{\dot{\gamma}}\right)+2 i\left(\overline{\mathcal{D}}^{\dot{\gamma}} G_{\beta} \dot{\delta}\right) \bar{M}_{\dot{\gamma} \dot{\delta}}-4 i W_{\beta}{ }^{\gamma \delta} M_{\gamma \delta},} \\
& {\left[\mathcal{D}_{\alpha}, \mathcal{D}_{\beta \dot{\beta}}\right]=i \varepsilon_{\alpha \beta}\left(\bar{R} \overline{\mathcal{D}}_{\dot{\beta}}+G_{\dot{\beta}}^{\gamma} \mathcal{D}_{\gamma}\right)} \\
& +i\left(\overline{\mathcal{D}}_{\dot{\beta}} \bar{R}\right) M_{\alpha \beta}-i \varepsilon_{\alpha \beta}\left(\mathcal{D}^{\gamma} G_{\dot{\beta}}^{\delta}\right) M_{\gamma \delta}+2 i \varepsilon_{\alpha \beta} \bar{W}_{\dot{\beta}}^{\dot{\gamma} \dot{\delta}} \bar{M}_{\dot{\gamma} \dot{\delta}}, \\
& {\left[\mathcal{D}^{\beta}, \mathcal{D}_{\beta \dot{\beta}}\right]=2 i\left(\bar{R} \overline{\mathcal{D}}_{\dot{\beta}}+G_{\dot{\beta}}^{\gamma} \mathcal{D}_{\gamma}\right)-2 i\left(\mathcal{D}^{\gamma} G_{\dot{\beta}}^{\delta}\right) M_{\gamma \delta}+4 i \bar{W}_{\dot{\beta}}^{\dot{\dot{\gamma}} \dot{\delta}} \bar{M}_{\dot{\gamma} \dot{\delta}}, \quad(6.88 \mathrm{~m})}
\end{aligned}
$$




$$
\begin{aligned}
{\left[\mathcal{D}^{2}, \overline{\mathcal{D}}^{2}\right]=} & {\left[\mathcal{D}^{2}, \overline{\mathcal{D}}_{\dot{\alpha}}\right] \overline{\mathcal{D}}^{\dot{\alpha}}-\overline{\mathcal{D}}^{\dot{\alpha}}\left[\mathcal{D}^{2}, \overline{\mathcal{D}}_{\dot{\alpha}}\right] } \\
= & 8 i G_{\alpha \dot{\alpha}} \mathcal{D}^{\alpha \dot{\alpha}}-4 i \mathcal{D}_{\alpha \dot{\alpha}}\left[\mathcal{D}^{\alpha}, \overline{\mathcal{D}}^{\dot{\alpha}}\right] \\
& -4\left(\mathcal{D}^{\alpha} R\right) \mathcal{D}_{\alpha}+4\left(\overline{\mathcal{D}}_{\dot{\alpha}} \bar{R}\right) \overline{\mathcal{D}}^{\dot{\alpha}} \\
& -8 R \mathcal{D}^{2}+8 \bar{R} \overline{\mathcal{D}}^{2} \\
& -8\left(\mathcal{D}^{\gamma} G^{\delta} \dot{\alpha}\right) \overline{\mathcal{D}}^{\dot{\alpha}} M_{\gamma \delta}+8\left(\overline{\mathcal{D}}^{\dot{\gamma}} G^{\alpha \dot{\delta}}\right) \mathcal{D}_{\alpha} \bar{M}_{\dot{\gamma} \dot{\delta}} \\
& -16 W^{\alpha \gamma \delta} \mathcal{D}_{\alpha} M_{\gamma \delta}+16 \bar{W}_{\dot{\alpha}}^{\dot{\alpha} \dot{\gamma}} \overline{\mathcal{D}}^{\dot{\alpha}} \bar{M}_{\dot{\gamma} \dot{\delta}} \\
& -8\left(\mathcal{D}^{\beta} W_{\beta}^{\gamma \delta}\right) M_{\gamma \delta}+8\left(\overline{\mathcal{D}}_{\dot{\alpha}} \bar{W}^{\dot{\alpha} \dot{\gamma} \dot{\delta}}\right) \bar{M}_{\dot{\gamma} \dot{\delta}} .
\end{aligned}
$$

In minimal SUGRA $R$ and $W_{\alpha \beta \gamma}$ are chiral fields, $G_{\alpha \dot{\alpha}}$ is real.

$$
\begin{aligned}
G_{a} & =\bar{G}_{a}, \\
\overline{\mathcal{D}}_{\dot{\alpha}} R & =0, \\
\overline{\mathcal{D}}_{\dot{\alpha}} W_{\alpha \beta \gamma} & =0,
\end{aligned}
$$

The remaining identities also simplify dramatically,

$$
\begin{aligned}
\overline{\mathcal{D}}^{\dot{\alpha}} G_{\alpha \dot{\alpha}} & =\mathcal{D}_{\alpha} R \\
\mathcal{D}^{\alpha} G_{\alpha \dot{\alpha}} & =\overline{\mathcal{D}}_{\dot{\alpha}} \bar{R} \\
\mathcal{D}^{\gamma} W_{\alpha \beta \gamma} & =\frac{i}{2} \mathcal{D}_{\alpha}{ }^{\dot{\alpha}} G_{\beta \dot{\alpha}}+\frac{i}{2} \mathcal{D}_{\beta}^{\dot{\alpha}} G_{\alpha \dot{\alpha}}
\end{aligned}
$$

Some trivial consequences of the above identities are

$$
\begin{aligned}
\overline{\mathcal{D}}_{\dot{\alpha}} G^{\alpha \dot{\alpha}}= & -\mathcal{D}^{\alpha} R, \\
\mathcal{D}_{\alpha} G^{\alpha \dot{\alpha}}= & -\overline{\mathcal{D}}^{\dot{\alpha}} \bar{R} \\
\mathcal{D}^{\alpha \dot{\alpha}} G_{\alpha \dot{\alpha}}= & \frac{i}{2}\left(\mathcal{D}^{2} R-\overline{\mathcal{D}}^{2} \bar{R}\right) \\
\left(\mathcal{D}^{2} \lambda\right)\left(\overline{\mathcal{D}}^{2} \bar{\lambda}\right)= & 4 G_{\alpha \dot{\alpha}}\left(\mathcal{D}^{\alpha} \lambda\right)\left(\overline{\mathcal{D}}^{\dot{\alpha}} \bar{\lambda}\right)+8\left(\mathcal{D}_{\alpha \dot{\alpha}} \lambda\right)\left(\mathcal{D}^{\alpha \dot{\alpha}} \bar{\lambda}\right) \\
& +(\text { total derivative }) .
\end{aligned}
$$

\subsubsection{Chiral Projector and d'Alembertian}

As a consequence of (6.88c) as long as $R \neq 0, \overline{\mathcal{D}}^{2} U$ is no longer chiral ( $U$ being an arbitrary superfield). But for tensor superfields carrying no 
dotted indices the following operator gives a covariantly chiral superfield.

$$
\overline{\mathcal{D}}_{\dot{\alpha}}\left(\overline{\mathcal{D}}^{2}-4 R\right) U_{\alpha_{1} \ldots \alpha_{n}}=0 \quad \forall \text { undotted tensor superfield } U
$$

Evidently the flat space limit, $R \rightarrow 0$ restores the usual chirality property of $\overline{\mathcal{D}}^{2} U$.

Since chiral scalar superfields will play an important rôle in this thesis, the commutators (6.88) acting on chiral scalar fields are worked out explicitly in appendix E. The combination $\overline{\mathcal{D}}^{2}-4 R$ is also known as the chiral projector .

(anti-)chiral From the chiral projector a generalisation of the d'Alembert operator d'Alembertian to the space of (anti-)chiral superfields can be given. The (anti-)chiral d'Alembertian $\square_{+}\left(\square_{-}\right)$is defined by

$$
\begin{aligned}
& \square_{+}:=\left(\mathcal{D}^{a}+i G^{a}\right) \mathcal{D}_{a}+\frac{1}{4}\left(R \mathcal{D}^{\alpha}+\left(\mathcal{D}^{\alpha} R\right)\right) \mathcal{D}_{\alpha}, \\
& \square_{-}:=\left(\mathcal{D}^{a}-i G^{a}\right) \mathcal{D}_{a}+\frac{1}{4}\left(\bar{R} \overline{\mathcal{D}}_{\dot{\alpha}}+\left(\overline{\mathcal{D}}_{\dot{\alpha}} \bar{R}\right)\right) \overline{\mathcal{D}}^{\dot{\alpha}},
\end{aligned}
$$

and maps to (anti-)chiral fields as long as it acts on (anti-)chiral fields. In this case $\square_{+}\left(\square_{-}\right)$may be rewritten in the following manner,

$$
\begin{aligned}
& \square_{+} \lambda=\frac{1}{16}\left(\overline{\mathcal{D}}^{2}-4 R\right) \mathcal{D}^{2} \lambda, \\
& \square_{-} \bar{\lambda}=\frac{1}{16}\left(\mathcal{D}^{2}-4 \bar{R}\right) \overline{\mathcal{D}}^{2} \bar{\lambda}
\end{aligned}
$$

which makes manifest the (anti-)chirality property.

Also note that $\overline{\mathcal{D}}^{2} \mathcal{D}^{2} \lambda=16\left(\square_{+}+\frac{1}{4} R \mathcal{D}^{2}\right) \lambda$.

\subsubsection{Super-Weyl Transformations}

The condition $T_{\alpha}=0$ is only invariant under a subset of the mixed superWeyl/local $\mathrm{U}(1)_{R}$ transformations discussed in Section 6.3.4. To ensure that 0 maps to 0 under those transformations, from

$$
0=T_{\alpha} \mapsto L T_{\alpha}+L \mathcal{D}_{\alpha} \ln \left(L^{4} \bar{L}^{2}\right)=0,
$$


the condition $\mathcal{D}_{\alpha} \ln \left(L^{4} \bar{L}^{2}\right)=0$ is read off. Consequently the parameter $L$ is restricted to be of the form

$$
\begin{aligned}
L & =\exp \left(\frac{1}{2} \sigma-\bar{\sigma}\right), \quad \overline{\mathcal{D}}_{\dot{\alpha}} \sigma=\mathcal{D}_{\alpha} \bar{\sigma}=0, \\
\bar{L} & =\exp \left(\frac{1}{2} \bar{\sigma}-\sigma\right)
\end{aligned}
$$

The minimal supergravity fields transform according to

$$
\begin{aligned}
\mathcal{D}_{\alpha}^{\prime} & =L \mathcal{D}_{\alpha}-2\left(\mathcal{D}^{\beta} L\right) M_{\alpha \beta}, \\
R^{\prime} & =-\frac{1}{4}\left(\overline{\mathcal{D}}^{2}-4 R\right) \bar{L}^{2}, \\
G_{\alpha \dot{\alpha}}^{\prime} & =L \bar{L} G_{\alpha \dot{\alpha}}+\frac{1}{2} \bar{L} \mathcal{D}_{\alpha} \overline{\mathcal{D}}_{\dot{\alpha}} L-\frac{1}{2} L \overline{\mathcal{D}}_{\dot{\alpha}} \mathcal{D}_{\alpha} \bar{L} \\
W_{\alpha \beta \gamma}^{\prime} & =L^{2} \bar{L} W_{\alpha \beta \gamma},
\end{aligned}
$$

or in terms of $\sigma$ and $\bar{\sigma}$,

$$
\begin{aligned}
\mathcal{D}_{\alpha}^{\prime} & =\mathrm{e}^{\frac{1}{2} \sigma-\bar{\sigma}}\left(\mathcal{D}_{\alpha}-\left(\mathcal{D}^{\beta} \sigma\right) M_{\alpha \beta}\right), \\
R^{\prime} & =-\frac{1}{4} \mathrm{e}^{-2 \sigma}\left[\left(\overline{\mathcal{D}}^{2}-4 R\right) \mathrm{e}^{\bar{\sigma}}\right], \\
G_{\alpha \dot{\alpha}}^{\prime} & =\mathrm{e}^{-(\sigma+\bar{\sigma}) / 2}\left[G_{\alpha \dot{\alpha}}+\frac{1}{2}\left(\mathcal{D}_{\alpha} \sigma\right)\left(\overline{\mathcal{D}}_{\dot{\alpha}} \bar{\sigma}\right)+i\left(\mathcal{D}_{\alpha \dot{\alpha}}(\bar{\sigma}-\sigma)\right)\right], \\
W_{\alpha \beta \gamma}^{\prime} & =\mathrm{e}^{-3 \sigma / 2} W_{\alpha \beta \gamma} .
\end{aligned}
$$

Formulating $\bar{T}_{\dot{\alpha}}=0$ in terms of prepotentials (6.85) yields the impor- chiral tant equation

$$
\bar{E}_{\dot{\alpha}} \varphi=0, \quad \varphi^{3}:=E^{-1} \bar{F}^{-2}\left(1 \cdot \mathrm{e}^{\overleftarrow{\bar{W}}}\right)^{-1}
$$

where the exponent of " 3 " is for convenience as is seen in the next equation. Since for any scalar $\overline{\mathcal{D}}_{\dot{\alpha}} \equiv \bar{E}_{\dot{\alpha}}$, the field $\varphi$ is chiral and transforms under generalised super-Weyl transformations into

$$
\varphi^{3} \mapsto\left[(L \bar{L})^{-2} E^{-1}\right]\left[\bar{L}^{-2} \bar{F}^{-2}\right]\left(1 \cdot \mathrm{e}^{\overleftarrow{\bar{W}}}\right)^{-1}=L^{-2} \bar{L}^{-4} \varphi^{3}=\left(\mathrm{e}^{\sigma} \varphi\right)^{3}
$$

This makes $\varphi$ the compensating field for super-Weyl transformations. Accordingly it is called chiral compensator. 


\subsubsection{Chiral Representation and Integration Rule}

Performing the picture changing operation

$$
\begin{aligned}
\tilde{V} & =\mathrm{e}^{-\bar{W}} V, \\
\tilde{\mathcal{D}}_{A} & =\mathrm{e}^{-\bar{W}} \mathcal{D}_{A} \mathrm{e}^{\bar{W}}=\tilde{E}_{A}^{M} \partial_{M}+\frac{1}{2} \tilde{\Omega}_{A}^{b c} M_{b c},
\end{aligned}
$$

and additionally going to the gauge $N_{\alpha}{ }^{\mu}=\delta_{\alpha}{ }^{\mu}$ introduces the so-called chiral representation. The important feature of the chiral representation is that the spinorial vielbein $\tilde{\bar{E}}_{\dot{\alpha}}=-\bar{F} \bar{\partial}_{\dot{\alpha}}$ takes a most simple form, while $\tilde{E}_{\alpha}$ and complex conjugation are more complicated than in the vector representation used so far. The determinant of the vierbein becomes

$$
\tilde{E}^{-1}=\left(E^{-1} \mathrm{e}^{-\overleftarrow{W}}\right)
$$

such that

$$
\int d^{8} z \tilde{E}^{-1} \tilde{\mathscr{L}}=\int d^{8} z\left(E^{-1} \mathrm{e}^{-\overleftarrow{W}}\right) \mathrm{e}^{-W} \stackrel{\mathscr{L}^{(6.72)}}{=} \int d^{8} z E^{-1} \mathscr{L}
$$

In chiral representation, equations (6.109) and (6.86) read

$$
\begin{aligned}
\tilde{\varphi}^{3} \bar{F}^{2} & =\tilde{E}^{-1}, \\
\tilde{R} & =\frac{1}{4} \bar{\partial}_{\dot{\mu}} \bar{\partial}^{\dot{\mu}} \bar{F}^{2},
\end{aligned}
$$

which combined yield

$$
\begin{aligned}
\tilde{\varphi}^{3} \tilde{R} & =\frac{1}{4} \bar{\partial}_{\dot{\mu}} \bar{\partial}^{\dot{\mu}} \tilde{E}^{-1}, \\
\Longrightarrow \tilde{\varphi}^{3} \tilde{\mathscr{L}}_{c} & =\frac{1}{4} \bar{\partial}_{\dot{\mu}} \bar{\partial}^{\dot{\mu}}\left(\frac{\tilde{E}^{-1}}{\tilde{R}} \tilde{\mathscr{L}}_{c}\right) .
\end{aligned}
$$

This gives the important chiral integration rule

$$
\int d^{6} z \tilde{\varphi}^{3} \tilde{\mathscr{L}}_{c}=\int d^{8} z \frac{\tilde{E}^{-1}}{\tilde{R}} \tilde{\mathscr{L}}_{c} \stackrel{(6.114)}{=} \int d^{8} z \frac{E^{-1}}{R} \mathscr{L},
$$

due to $d^{2} \bar{\theta}=\frac{1}{4} \bar{\partial}_{\dot{\mu}} \bar{\partial}^{\dot{\mu}}$. 


\subsection{Component Expansion}

\subsubsection{Superfields and First Order Operators}

In supergravity as opposed to flat supersymmetry, the (non-linearised) components of a superfield are given in terms of covariant derivatives $\mathcal{D}_{\alpha}$ and $\overline{\mathcal{D}}_{\dot{\alpha}}$ and are in one-to-one correspondence to the coefficients in the usual $\theta, \bar{\theta}$ expansion of a superfield.

$$
\begin{array}{rrr}
\left.f\right|_{0} & \left.\mathcal{D}_{\alpha} f\right|_{0} & \left.\overline{\mathcal{D}}_{\dot{\alpha}} f\right|_{0} \\
-\left.\frac{1}{4} \mathcal{D}^{2} f\right|_{0} & -\left.\frac{1}{4} \overline{\mathcal{D}}^{2} f\right|_{0} & \left.\frac{1}{2}\left[\mathcal{D}_{\alpha}, \overline{\mathcal{D}}_{\dot{\alpha}}\right] f\right|_{0} \\
-\left.\frac{1}{4} \mathcal{D}_{\alpha} \overline{\mathcal{D}}^{2} f\right|_{0} & -\left.\frac{1}{4} \overline{\mathcal{D}}_{\alpha} \mathcal{D}^{2} f\right|_{0} & -\left.\frac{1}{32}\left\{\mathcal{D}^{2}, \overline{\mathcal{D}}^{2}\right\} f\right|_{0}
\end{array}
$$

Here, the notation

$$
\left.f\right|_{0}:=f(x, \theta=0, \bar{\theta}=0)
$$

has been introduced.

For arbitrary superfields $f_{1}$ and $f_{2}$, it holds

$$
\left.\left(f_{1} f_{2}\right)\right|_{0}=\left.\left.f_{1}\right|_{0} f_{2}\right|_{0},
$$

which obviously can no longer be true when $f_{1}$ is an operator containing derivatives on anticommuting coordinates.

The space projection of a general first order differential operator

$$
\mathcal{O}=\mathcal{O}^{M}(z) \partial_{M}+\mathcal{O}^{a b}(z) M_{a b}
$$

is defined to be

$$
\left.\mathcal{O}\right|_{0}=\left.\mathcal{O}^{M}\right|_{0} \partial_{M}+\left.\mathcal{O}^{a b}\right|_{0} M_{a b}
$$

Acting with such an operator on an arbitrary superfield (with Lorentz 
indices of $f$ suppressed), one immediately sees that

$$
\begin{aligned}
\left.(\mathcal{O} f)\right|_{0} & =\left.\left(\mathcal{O}^{M} \partial_{M} f\right)\right|_{0}+\left.\left(\mathcal{O}^{a b} M_{a b} f\right)\right|_{0} \\
& =\left.\left.\mathcal{O}^{M}\right|_{0} \partial_{M} f\right|_{0}+\left.\left.\mathcal{O}^{a b}\right|_{0} M_{a b} f\right|_{0} \\
& =\left.\left(\left.\mathcal{O}\right|_{0} f\right)\right|_{0}
\end{aligned}
$$

is different from

$$
\left.\left.\mathcal{O}\right|_{0} f\right|_{0}=\left.\left.\mathcal{O}^{m}\right|_{0} \partial_{m} f\right|_{0}+\left.\left.\mathcal{O}^{a b}\right|_{0} M_{a b} f\right|_{0}
$$

Using pure superspace methods, it is possible (though tedious) to show, that in Wess-Zumino gauge the vector derivative has the following expansion,

$$
\left.\mathcal{D}_{\alpha \dot{\alpha}}\right|_{0}=\left.\nabla_{\alpha \dot{\alpha}}\right|_{0}+\left.\frac{1}{2} \Psi_{\alpha \dot{\alpha},}^{\beta} \mathcal{D}_{\beta}\right|_{0}+\left.\frac{1}{2} \bar{\Psi}_{\alpha \dot{\alpha}, \dot{\beta}} \overline{\mathcal{D}}^{\dot{\beta}}\right|_{0}
$$

with $\Psi$ the gaugino field strength. As a simple example, the expansion of $\mathcal{D}_{\alpha \dot{\alpha}} f$ is given,

$$
\begin{aligned}
\left.\left(\mathcal{D}_{\alpha \dot{\alpha}} f\right)\right|_{0} & =\left.\left(\left.\mathcal{D}_{\alpha \dot{\alpha}}\right|_{0} f\right)\right|_{0} \\
& =\nabla_{\alpha \dot{\alpha}}\left(\left.f\right|_{0}\right)+\frac{1}{2} \Psi_{\alpha \dot{\alpha},}^{\beta}\left(\left.\left(\mathcal{D}_{\beta} f\right)\right|_{0}\right)+\frac{1}{2} \bar{\Psi}_{\alpha \dot{\alpha}, \beta}\left(\left.\left(\overline{\mathcal{D}}^{\beta} f\right)\right|_{0}\right) .
\end{aligned}
$$

More complicated combination of the derivatives $\mathcal{D}_{\alpha}, \overline{\mathcal{D}}_{\dot{\alpha}}$ and $\mathcal{D}_{\alpha \dot{\alpha}}$ acting on a field require rearrangement such that the leftmost derivative is of vector type. Then the above rule (with $f$ containing the remaining derivatives) can be used to recursively reduce the superspace derivatives to space-time covariant derivatives $\nabla_{\alpha \dot{\alpha}}$ until only expressions containing component combinations (6.120) of the spinorial derivatives are left over. Due to the three-folding caused by each application of (6.128), let alone the required rearrangement of vector derivatives to the left, even terms with a relatively small number of derivatives may grow dramatically. The situation is (slightly) better when one is not interested in terms containing the gaugino field strength. Therefore, the operator $\left.\right|_{\mathrm{b}}$ shall denote spacetime projection while simultaneously neglecting all gravitational fermionic and auxiliary fields. 


\subsubsection{Supergravity Fields}

The derivation of the component expansion in Wess-Zumino gauge is rather involved and only the final expression shall be reproduced here. The real part of the prepotential $W$ can be gauged away, but requiring instead the condition

$$
\exp \left(\bar{W}^{n} \partial_{n}\right) x^{m}=x^{m}+i \mathcal{H}^{m}(x, \theta, \bar{\theta}) \quad \mathcal{H}^{m}=\overline{\mathcal{H}}^{m}
$$

defines the gravitational Wess-Zumino gauge, also called gravitational superfield gauge. In this gauge, the gravitational degrees of freedom are encoded in the gravitational superfield $\mathcal{H}^{m}$ and the chiral compensator $\hat{\varphi}(x, \theta)$.

$$
\begin{aligned}
\mathcal{H}^{m} & =\theta \sigma^{a} \bar{\theta} e_{a}{ }^{m}+i \bar{\theta}^{2} \theta^{\alpha} \psi_{\alpha}^{m}{ }_{\alpha}-i \theta^{2} \bar{\theta}_{\dot{\alpha}} \bar{\Psi}^{m \dot{\alpha}}+\theta^{2} \bar{\theta}^{2} A^{m} \\
\hat{\varphi}^{3} & =e^{-1}\left(1-2 i \theta \sigma_{a} \bar{\Psi}^{a}+\theta^{2} B\right) \quad \hat{\varphi}=\mathrm{e}^{-\bar{W}} \varphi \\
\hat{\bar{\varphi}}^{3} & =e^{-1}\left(1-2 i \bar{\theta} \tilde{\sigma}_{a} \Psi^{a}+\bar{\theta}^{2} \bar{B}\right)
\end{aligned}
$$

In Wess-Zumino gauge, the spinorial semi-covariant vierbein fields (6.87) coincide with the partial derivatives and can therefore be used to extract the components of the above gravitational superfields just as in flat supersymmetry.

The spinorial semi-covariant vierbein fields $\hat{E}_{\alpha}, \hat{\bar{E}}_{\dot{\alpha}}$ were defined by just pulling out a factor of $F$ from the covariant spinorial derivatives $\mathcal{D}_{\alpha}$, $\overline{\mathcal{D}}_{\dot{\alpha}}$. In addition without proof, for the prepotential $F$ it holds

$$
\left.F\right|_{0}=1, \quad \hat{E}_{\alpha} F=-\frac{i}{2} \bar{\Psi}^{\alpha \dot{\beta} \dot{\beta}}
$$

such that

$$
\begin{aligned}
\left.\mathcal{D}_{\alpha} \mathcal{O}\right|_{0} & =\left.\hat{E}_{\alpha} \mathcal{O}\right|_{0}, \\
-\left.\frac{1}{4} \mathcal{D}^{2} \mathcal{O}\right|_{0} & =-\left.\frac{1}{4} \hat{E}^{2} \mathcal{O}\right|_{0}+\frac{i}{2} \bar{\Psi}_{\dot{\beta},\left.{ }^{\alpha} \mathcal{D}_{\alpha} \mathcal{O}\right|_{0} .}
\end{aligned}
$$

This allows to write down the chiral compensator's components in terms 
of covariant derivatives

$$
\begin{aligned}
\left.\varphi^{3}\right|_{0} & =e^{-1} \\
\left.\mathcal{D}_{\alpha} \varphi^{3}\right|_{0} & =-2 i e^{-1}\left(\sigma^{a} \bar{\Psi}_{a}\right)_{\alpha}, \\
-\left.\frac{1}{4} \mathcal{D}^{2} \varphi^{3}\right|_{0} & =e^{-1}(B-\bar{\Psi} \tilde{\sigma} \sigma \bar{\Psi}),
\end{aligned}
$$

where $\bar{\Psi} \tilde{\sigma} \sigma \bar{\Psi}=-\bar{\Psi}_{\dot{\beta}}^{\alpha},{ }^{\dot{\beta}} \bar{\Psi}_{\alpha \dot{\gamma}}{ }^{\dot{\gamma}}$. In other words

$$
\begin{aligned}
\left.\varphi\right|_{0} & =e^{-1 / 3} \\
\left.\mathcal{D}_{\alpha} \varphi\right|_{0} & =-\frac{2}{3} i e^{-1 / 3}\left(\sigma^{a} \bar{\Psi}_{a}\right)_{\alpha} \\
-\left.\frac{1}{4} \mathcal{D}^{2} \varphi\right|_{0} & =\frac{1}{3} e^{-1 / 3}\left(B-\frac{1}{3} \bar{\Psi} \tilde{\sigma} \sigma \bar{\Psi}\right)
\end{aligned}
$$

For the chiral supertorsion component:

$$
\begin{aligned}
\left.\bar{R}\right|_{0}= & \frac{1}{3} \mathbf{B}, \quad \mathbf{B}=B+\frac{1}{2} \bar{\Psi}^{a} \tilde{\sigma}_{a} \sigma_{b} \bar{\Psi}^{b}+\frac{1}{2} \bar{\Psi}^{a} \bar{\Psi}_{a}, \\
\left.\overline{\mathcal{D}}_{\dot{\alpha}} \bar{R}\right|_{0}= & \frac{4}{3} \bar{\Psi}_{\dot{\alpha} \dot{\beta}}, \dot{\beta}+\frac{i}{3} \mathbf{B} \Psi_{\dot{\alpha}, \beta}^{\beta}, \\
\left.\overline{\mathcal{D}}^{2} \bar{R}\right|_{0}= & \frac{2}{3}\left(\mathcal{R}+\frac{i}{2} \varepsilon^{a b c d} \mathcal{R}_{a b c d}\right)+\frac{8}{9} \overline{\mathbf{B}} \mathbf{B} \\
& -\frac{2}{9} \mathbf{B}\left(\Psi^{a} \sigma_{a} \tilde{\sigma}_{b} \Psi^{b}+\Psi^{a} \Psi_{a}\right) \\
& +\left.i \overline{\mathcal{D}}_{\dot{\alpha}} \bar{R}\right|_{0}\left(\tilde{\sigma}_{b} \Psi^{b}\right)^{\dot{\alpha}}+\left.\frac{2 i}{3} \Psi^{\alpha \dot{\alpha}, \beta} \mathcal{D}_{\{\alpha} G_{\beta\}, \dot{\alpha}}\right|_{0},
\end{aligned}
$$

where $\mathcal{R}$ denotes the Ricci scalar, tensor or Riemann tensor, respectively.

For the real supertorsion component:

$$
\begin{aligned}
& \left.G_{a}\right|_{0}=\frac{4}{3} \mathbf{A}_{a} \\
& \mathbf{A}_{a}=A^{a}+\frac{1}{8} \varepsilon^{a b c d} \mathcal{C}_{b c d}-\frac{1}{4}\left(\Psi_{a} \sigma_{b} \bar{\Psi}^{b}+\Psi^{b} \sigma_{b} \bar{\Psi}_{a}\right) \\
& -\frac{1}{2} \Psi^{b} \sigma_{a} \bar{\Psi}_{b}+\frac{i}{8} \varepsilon^{a b c d} \Psi_{b} \sigma_{c} \bar{\Psi}_{d} \\
& \left.\overline{\mathcal{D}}_{\{\dot{\alpha}} G_{\dot{\beta}\}}^{\beta}\right|_{0}=-2 \Psi_{\dot{\alpha} \dot{\beta},}{ }^{\beta}+\frac{i}{3} \overline{\mathbf{B}} \bar{\Psi}_{\{\dot{\alpha}, \dot{\beta}\}}^{\beta}, \\
& \left.\overline{\mathcal{D}}_{\{\dot{\alpha}} \mathcal{D}^{\{\gamma} G^{\delta\}}{ }_{\dot{\beta}\}}\right|_{0}=2 E_{\dot{\alpha} \dot{\beta}}^{\gamma \delta}+2 i \Psi^{\{\gamma}{ }_{\{\dot{\alpha}},\left.{ }^{\delta\}} \overline{\mathcal{D}}_{\dot{\beta}\}} \bar{R}\right|_{0}-\left.i \Psi_{\alpha\{\dot{\alpha},}{ }^{\alpha} \mathcal{D}^{\{\gamma} G^{\delta\}}{ }_{\dot{\beta}\}}\right|_{0} \\
& +\left.2 i \bar{\Psi}_{\alpha\{\dot{\alpha}, \dot{\beta}\}} W^{\alpha \gamma \delta}\right|_{0}+\frac{2}{3} \mathbf{B}\left(\tilde{\sigma}^{a b}\right)_{\dot{\alpha} \dot{\beta}} \Psi_{a}^{\gamma} \Psi_{b}^{\delta},
\end{aligned}
$$


with

$$
\begin{aligned}
E_{a b} & :=\frac{1}{4}\left(2 \tilde{\mathcal{R}}_{a b}+\frac{i}{2}\left(\varepsilon_{a c d e} \mathcal{R}_{b}^{c d e}+\varepsilon_{b c d e} \mathcal{R}_{a}^{c d e}-\frac{1}{2} \eta_{a b} \varepsilon^{c d e f} \mathcal{R}_{c d e f}\right)\right) \\
\tilde{\mathcal{R}}_{a b} & :=\frac{1}{2}\left(\mathcal{R}_{a b}+\mathcal{R}_{b a}\right)-\frac{1}{4} \eta_{a b} \mathcal{R}=\mathcal{G}_{\{a b\}}+\frac{1}{4} \eta_{a b} \mathcal{R}
\end{aligned}
$$

\subsubsection{Full Superspace Integrals}

Using the chiral integration rule (6.119), any real superspace integral can be reduced to a chiral one.

$$
\begin{aligned}
S & =\int d^{8} z E^{-1} \mathscr{L} \\
& =\int d^{8} z \frac{E^{-1}}{R} \underbrace{\left(-\frac{1}{4}\right)\left(\overline{\mathcal{D}}^{2}-4 R\right) \mathscr{L}}_{=: \mathscr{L}_{c}}
\end{aligned}
$$

Then the following manipulations, which crucially depend on the semi- density formula covariant vierbein coinciding (6.87) with the partial derivatives in WessZumino gauge, lead to the density formula

$$
\begin{aligned}
S & =\int d^{6} z \hat{\varphi}^{3} \hat{\mathscr{L}}_{c}=\frac{1}{4} \int d^{4} x \partial^{\alpha} \partial_{\alpha}\left(\hat{\varphi}^{3} \hat{\mathscr{L}}_{c}\right)=-\left.\frac{1}{4} \int d^{4} x \hat{E}^{2}\left(\varphi^{3} \mathscr{L}_{c}\right)\right|_{0} \\
& =-\left.\left.\frac{1}{4} \int d^{4} x \varphi^{3}\right|_{0} \hat{E}^{2} \mathscr{L}_{c}\right|_{0}+\left.\left.2 \mathcal{D}^{\alpha} \varphi^{3}\right|_{0} \mathcal{D}_{\alpha} \mathscr{L}_{c}\right|_{0}+\left.\left.\hat{E}^{2} \varphi^{3}\right|_{0} \mathscr{L}_{c}\right|_{0} \\
& =\left.\left.\int d^{4} x \varphi^{3}\right|_{0}\left(-\frac{1}{4} \mathcal{D}^{2} \mathscr{L}_{c}\right)\right|_{0}-\left.\left.\frac{1}{4} \mathcal{D}^{\alpha} \varphi^{3}\right|_{0} \mathcal{D}_{\alpha} \mathscr{L}_{c}\right|_{0}+\left.B \mathscr{L}_{c}\right|_{0} .
\end{aligned}
$$

where $B=\mathbf{B}-\frac{1}{2} \bar{\Psi}^{a} \tilde{\sigma}_{a} \sigma_{b} \bar{\Psi}^{b}-\frac{1}{2} \bar{\Psi}^{a} \bar{\Psi}_{a}=-\frac{1}{4} \mathcal{D}^{2} \varphi^{3}+e^{-1} \bar{\Psi} \tilde{\sigma} \sigma \bar{\Psi}$. 

I adore simple pleasures. They are the last refuge of

the complex.

Oscar Wilde, "The Picture of Dorian Gray"

\section{Chapter 7}

\section{Space-Time Dependent \\ Couplings}

§7.1 Weyl Transformations, 122. §7.1.1 Conformal Killing Equation, 122. §7.1.2 Conformal Algebra in $d>2,123$. $\S 7.1 .3$ Weyl Transformations of the Riemann Tensor, 124. §7.1.4 Weyl Covariant Differential Operators, 125. §7.2 Zamolodchikov's $c$-Theorem in Two Dimensions, 127. $\S 7.3$ Conformal Anomaly in Four Dimensions, 129. \$7.4 Local RG Equation and the $c$-Theorem, 130. §7.4.1 $a$-Theorem, 133.

This Chapter is meant to give a short introduction into the space-time dependent couplings technique and its application to a proof of Zamolodchikov's $c$-theorem in two dimensions. Additionally the four dimensional trace anomaly and some of the problems encountered when trying to extend the theorem to four dimensions are discussed. 


\subsection{Weyl Transformations}

\subsubsection{Conformal Killing Equation}

A Weyl transformation is a rescaling of the metric by a space-time dependent factor

$$
g_{m n} \mapsto \mathrm{e}^{-2 \sigma} g_{m n} .
$$

Upon restriction to flat space these transformations generate the conformal group, which locally preserves angles.

Using

$$
\begin{aligned}
\delta g_{m n} & =-2 \sigma g_{m n}, \\
\delta x^{m} & =\xi^{m} \\
\delta d x^{m} & =\left(\partial_{n} \xi^{m}\right) d x^{n},
\end{aligned}
$$

the requirement of invariance of the line element

$$
\delta\left(d s^{2}\right) \stackrel{!}{=} 0=\left[-2 \sigma g_{m n}+\partial_{m} \xi_{n}+\partial_{n} \xi_{m}\right] d x^{m} d x^{n}
$$

conformal Killing vector amounts to the conformal Killing vector equation

$$
\begin{aligned}
\partial_{m} \xi_{n}+\partial_{n} \xi_{m} & =\frac{2}{d} \partial_{k} \xi^{k} g_{m n}, \\
\sigma & =\frac{1}{d} \partial_{k} \xi^{k}
\end{aligned}
$$

where $d$ is the dimension of space time.

Under (7.2), the action transforms as follows,

$$
\begin{aligned}
\delta S & =\int d^{d} x \frac{\delta S}{\delta g_{m n}} \delta g_{m n} \\
& =\int d^{d} x\left[-\frac{1}{2} T^{m n}\right]\left[-2 \sigma g_{m n}\right],
\end{aligned}
$$

which demonstrates that for conformal invariance the trace of the energymomentum tensor has to vanish.

As an aside, in two dimensions after Wick rotation the conformal Cauchy-Riemann Killing vector equation becomes the Cauchy-Riemann system, such that conformal transformations are given by holomorphic or antiholomorphic 


\begin{tabular}{|c|c|c|}
\hline \multicolumn{3}{|c|}{ Conformal Transformations } \\
\hline Name & Group Element & Generator \\
\hline translations & $x^{a} \mapsto x^{a}+a^{a}$ & $P_{a}$ \\
\hline$\left(\right.$ Lorentz $\left.^{*}\right)$ rotations & $x^{a} \mapsto \Lambda_{b}^{a} x^{b}$ & $M_{a b}$ \\
\hline dilation & $x^{a} \mapsto \lambda x^{a}$ & $D$ \\
\hline $\mathrm{SCT}^{* *}$ & $x^{a} \mapsto \frac{x^{a}+b^{a} x^{2}}{\Omega_{\mathrm{SCR}}(x)}$ & $K_{a}$ \\
\hline
\end{tabular}

Table 7.1: Finite Conformal Transformations

functions. Decomposing these functions by a Laurent expansion demonstrates that the two dimensional conformal group has infinitely many generators, which form the Witt/Virasoro algebra.

The four dimensional case is generic and will be discussed below.

\subsubsection{Conformal Algebra in $d>2$}

In $d>2$ dimensions in Minkowski space, infinitesimal conformal transformations are given by

$$
\xi^{a}(x)=a^{a}+\omega^{a b} x_{b}+\lambda x^{a}+\left(x^{2} b^{a}-2 x^{a} x_{b} b^{b}\right)
$$

with the corresponding generators

$$
\delta_{C}=i a^{a} P_{a}+i \omega^{a b} M_{a b}+i \lambda D+i b^{a} K_{a},
$$

which form the conformal algebra

$$
\begin{array}{rlrl}
{\left[M_{a b}, P_{c}\right]} & =-2 i P_{[a} \eta_{b] c}, & {\left[M_{a b}, K_{c}\right]} & =-2 i K_{[a} \eta_{b] c}, \\
{\left[D, P_{a}\right]} & =-i P_{a}, \quad & {\left[D, K_{a}\right]} & =i K_{a}, \\
{\left[D, M_{a b}\right]} & =0, & {\left[P_{a}, K_{b}\right]} & =2 i\left(M_{a b}-\eta_{a b} D\right), \\
{\left[M_{a b}, M_{c d}\right]} & =2 i\left(\eta_{a[c} M_{d] b}-\eta_{b[c} M_{d] a}\right)
\end{array}
$$

This can be identified with the algebra $\mathfrak{s o}(d, 2)$ by defining a suitable 
$(d+2) \times(d+2)$ matrix

$$
M_{\hat{m} \hat{n}}:=\left(\begin{array}{ccc}
M_{m n} & \frac{1}{2}\left(K_{m}-P_{m}\right) & \frac{1}{2}\left(K_{m}+P_{m}\right) \\
-\frac{1}{2}\left(K_{m}-P_{m}\right) & 0 & -D \\
-\frac{1}{2}\left(K_{m}+P_{m}\right) & D & 0
\end{array}\right)
$$

and choosing $\eta_{\hat{m} \hat{n}}=\operatorname{diag}\left(\eta_{m n}, 1,-1\right)$ as metric. As an aside, the $d$-dimensional conformal algebra is identical to the $(d+1)$-dimensional $\mathfrak{a} \mathfrak{d} \mathfrak{s}$ algebra

$$
\mathfrak{c} \mathfrak{f}_{d} \equiv \mathfrak{a} \mathfrak{d} \mathfrak{s}_{d+1} \equiv \mathfrak{s o}(2, d)
$$

finite The finite transformations corresponding to the infinitesimal solutions transformations (7.6) are shown in Figure 7.1 , where $\Omega_{\mathrm{SCT}}(x):=1-\vec{b} \cdot \vec{x}+b^{2} \vec{x}^{2}$ is the scale factor $\Omega$ of the metric for special conformal transformations, and $\vec{a} \cdot \vec{b}$ has been used as a short-hand for $\eta_{m n} a^{m} b^{n}$.

\subsubsection{Weyl Transformations of the Riemann Tensor}

Since superspace supergravity is described using a tangent space formulation, which has the additional advantage of a metric $\delta\left[\eta_{a b}\right]=0$ invariant under Weyl transformations, the transformational behaviour of the Riemann $\mathcal{R}_{a b c d}$ and Weyl $C_{a b c d}$ tensor, Ricci tensor $\mathcal{R}_{a b}$ and scalar $\mathcal{R}$, and covariant derivative $\nabla$ under $\delta\left[g_{m n}\right]=-2 \sigma g_{m n}$ shall be given in terms of tangent space objects.

$$
\begin{aligned}
\delta\left[e_{a}{ }^{m}\right] & =\sigma e_{a}{ }^{m}, \\
\delta[\sqrt{-\operatorname{det} g}] & =\delta\left[\operatorname{det} e^{-1}\right]=-\sigma d \sqrt{-\operatorname{det} g}=-\sigma d \operatorname{det} e^{-1}, \\
\delta\left[\mathcal{R}_{c d}^{a b}\right] & =\delta_{[c}^{[a} \nabla^{b]} \nabla_{d]} \sigma+2 \sigma \mathcal{R}^{a b}{ }_{c d}, \\
\delta\left[\mathcal{R}_{a b c d}\right] & =\eta_{[c[a} \nabla_{b]} \nabla_{d]} \sigma+2 \sigma \mathcal{R}_{a b c d}, \\
\delta\left[\mathcal{R}_{a b}\right] & =\eta_{a b} \nabla^{2} \sigma+2 \nabla_{a} \nabla_{b} \sigma+2 \sigma \mathcal{R}_{a b}, \\
\delta[\mathcal{R}] & =6 \nabla^{2} \sigma+2 \sigma \mathcal{R},
\end{aligned}
$$

${ }^{*} \Lambda^{c}{ }_{a} \eta_{c d} \Lambda_{b}^{d}=\eta_{a b}$

** Special Conformal Transformation 


$$
\begin{aligned}
\delta\left[\mathcal{G}_{a b}\right] & =\delta\left[\mathcal{R}_{a b}\right]-\frac{1}{2} \eta_{a b} \delta[\mathcal{R}] \\
& =-2 \eta_{a b} \nabla^{2} \sigma+2 \nabla_{a} \nabla_{b} \sigma+2 \sigma \mathcal{G}_{a b}, \\
\delta\left[C_{a b c d}\right] & =2 \sigma C_{a b c d}, \\
\delta\left[\nabla_{a}\right] & =\sigma \nabla_{a}-\left(\nabla^{b} \sigma\right) M_{a b}, \quad M_{a b} V^{c}=\delta_{a}^{c} V_{b}-\delta_{b}^{c} V_{a}, \\
\delta\left[\nabla_{a} \lambda\right] & =\sigma \nabla_{a} \lambda, \\
\delta\left[\nabla^{2} \lambda\right] & =2 \sigma\left(\nabla^{2} \lambda\right)+(2-d)\left(\nabla^{a} \sigma\right)\left(\nabla_{a} \lambda\right),
\end{aligned}
$$

where $d$ is the space-time dimension, which from now on will be assumed to be equal to four.

\subsubsection{Weyl Covariant Differential Operators}

By definition a field $\psi$ is denoted conformally covariant if it transforms under Weyl transformations into $\mathrm{e}^{w \sigma} \psi$, that is homogeneously with Weyl weight $w$. In particular, it is interesting to have invariant expressions of the form

$$
\int d^{4} x e^{-1} \chi^{*} \Delta_{4-2 w} \psi
$$

with $\Delta_{4-2 w}$ a differential operator of order $4-2 w$ and $\psi, \chi$ are assumed to be Lorentz scalars.

The unique local, Weyl covariant differential operator acting on such fields $\psi$ and $\chi$ of Weyl weight 1 is given by

$$
\Delta_{2}=\nabla^{2}-\frac{1}{6} \mathcal{R}
$$

which can be easily verified using relations (7.11). It is however entertaining to derive this expression in a slightly different manner.

General relativity is not invariant under Weyl transformations as can be seen from the Einstein-Hilbert action transforming according to

$$
\int d^{4} x e^{-1} \mathcal{R} \mapsto \int d^{4} x e^{-1}\left[\mathrm{e}^{-2 \sigma} \mathcal{R}+6\left(\nabla^{a} \mathrm{e}^{-\sigma}\right)\left(\nabla_{a} \mathrm{e}^{-\sigma}\right)\right]
$$

Since Weyl transformations form an Abelian group, a parametrisation may be chosen where two consecutive transformations with parameters $\sigma_{1}$ and $\sigma_{2}$ correspond to a single Weyl transformation with parameter 
$\sigma_{1}+\sigma_{2}$. (Evidently $e_{a}{ }^{m} \mapsto \mathrm{e}^{\sigma} e_{a}^{m}$ is such a parametrisation.) Replacing the parameter of the first transformation by a field $\phi=e^{-\sigma_{1}}$ of Weyl weight 1 yields an invariant expression as can be seen from

$$
\mathrm{e}^{\sigma_{1}} e_{a}^{m}=\phi^{-1} e_{a}{ }^{m} \mapsto\left(\mathrm{e}^{-\sigma_{2}} \phi^{-1}\right)\left(\mathrm{e}^{\sigma_{2}} e_{a}{ }^{m}\right)=\phi^{-1} e_{a}{ }^{m} .
$$

Therefore, the following action is Weyl invariant

$$
\int d^{4} x e^{-1}\left[\phi^{2} \mathcal{R}+6\left(\nabla^{a} \phi\right)\left(\nabla_{a} \phi\right)\right]=6 \int d^{4} x e^{-1} \phi\left[\nabla^{2}-\frac{1}{6} \mathcal{R}\right] \phi
$$

and the operator $\Delta_{2}$ has been rederived.

compensator

In addition the important notion of a compensating field, here $\phi$, has been introduced. Compensating fields allow incorporating a symmetry into the formulation of a theory that originally was not part of it. An analogue procedure is needed to embed Poincaré supergravity into the Weyl invariant supergravity algebra by use of a so-called chiral compensator.

Unfortunately, the elegant method above does not lend itself to generalisations and clearly cannot be used to construct a conformally covariant operator for a field of vanishing Weyl weight. However a dimensional analysis can be used to write down a basis for such an operator and determine the prefactors from Weyl variation. The following operator due to Riegert [50] is the unique conformally covariant differential operator of fourth order, which because of its importance for this work will be given in several equivalent forms,

$$
\begin{aligned}
\Delta_{4} & :=\nabla^{4}+2 \mathcal{G}_{a b} \nabla^{a} \nabla^{b}+\frac{1}{3} \nabla^{a} \mathcal{R} \nabla_{a} \\
& =\nabla^{4}+2 \mathcal{G}_{a b} \nabla^{a} \nabla^{b}+\frac{1}{3}\left(\nabla^{a} \mathcal{R}\right) \nabla_{a}+\frac{1}{3} \mathcal{R} \nabla^{2} \\
& =\nabla^{4}+2 \mathcal{R}_{a b} \nabla^{a} \nabla^{b}+\frac{1}{3}\left(\nabla^{a} \mathcal{R}\right) \nabla_{a}-\frac{2}{3} \mathcal{R} \nabla^{2} \\
& =\nabla^{4}+2 \nabla^{a} \mathcal{R}_{a b} \nabla^{b}-\frac{2}{3}\left(\nabla^{a} \mathcal{R}\right) \nabla_{a}-\frac{2}{3} \mathcal{R} \nabla^{2}
\end{aligned}
$$

or partially integrated,

$$
\begin{aligned}
\lambda^{\prime} \Delta_{4} \lambda= & \left(\nabla^{2} \lambda\right)\left(\nabla^{2} \lambda^{\prime}\right)-2 \mathcal{G}_{a b}\left(\nabla^{a} \lambda\right)\left(\nabla^{b} \lambda^{\prime}\right) \\
& -\frac{1}{3} \mathcal{R}\left(\nabla^{a} \lambda\right)\left(\nabla_{a} \lambda^{\prime}\right)+(\text { total deriv. }) .
\end{aligned}
$$




\subsection{Zamolodchikov's $c$-Theorem in Two Di- mensions}

In a classical theory scale invariance is expected at the ultraviolet limit where particle masses may be neglected and at the infrared limit where massive particles decouple from the theory. In this sense the transition from UV to IR is irreversible in a classical theory. For simple theories scale invariance (which implies one additional symmetry generator) may be enough to establish conformal symmetry (which in two dimensions implies an infinite set of symmetry generators and is thus a much larger symmetry). At the quantum level, conformal invariance is often broken. Still there are many known examples of two dimensional theories which flow from one conformal fixed point in the UV to another one in the IR. In four dimensions the existence of conformal fixed points is much more difficult to establish.

The breaking of conformal invariance at the quantum level is induced by the introduction of a regulator during renormalisation, which creates a scale $\mu$ that leads to non-vanishing anomaly terms in the trace of the energy-momentum tensor.

Renormalisation group (RG) theory describes the change of the effective Hamiltonian of a theory during the change of scale. The breaking of $R G$ equation scale invariance is described by the RG equation

$$
\begin{gathered}
\mu \frac{d}{d \mu} W=\mu \frac{\partial}{\partial \mu} W+\beta^{i} \frac{\partial}{\partial \lambda^{i}} W=0, \\
\beta^{i}:=\mu \frac{\partial \lambda^{i}}{\partial \mu}, \\
W=W\left(\lambda^{i}, \mu\right),
\end{gathered}
$$

where $W$ is the generating functional of the connected Green's functions, which due to being a formal series expansion of physical observables is expected to be RG invariant, that is constant with respect to the scale $\mu$.

From a mathematical point of view, there is no reason a theory should not exhibit a complex flow behaviour. In particular the RG flow could approach a limit cycle, see Figure 7.1, possibly making the theory increase and decrease its number of degrees of freedom periodically while going to 


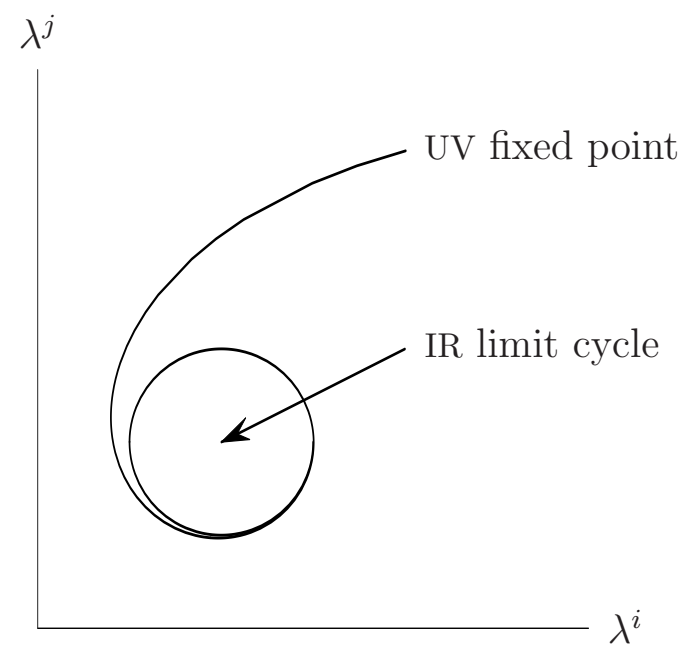

Figure 7.1: Limit Cycle in the Space of Couplings

lower and lower energies. Since this is certainly an unphysical behaviour, a natural question is under which conditions such a behaviour cannot be displayed by a quantum field theory.

A partial answer to this question was given by Zamolodchikov's fundamental theorem [43] in two dimensions, which states the irreversibility of RG flows connecting two fixed points in two dimensions.

Theorem 1 (Zamolodochikov 1986). "There exists a function $c(g)$ of the coupling constant $g$ in a $2 \mathrm{D}$ renormalisable field theory which decreases monotonically under the influence of a renormalisation group transformation. This function has constant values only at fixed points, where $c$ is the same as the central charge of a Virasoro algebra of the corresponding conformal field theory."

Therefore, it holds

$$
c_{U V} \geq c_{I R}
$$

where $c$ is the respective value of central charge at the infrared and ultraviolet. 


\subsection{Conformal Anomaly in Four Dimensions}

Due to its elegance and simplicity, the two-dimensional $c$-theorem was hoped to soon be generalised to four dimensions, but an accepted proof is outstanding for 20 years.

The first obstacle that arises is the question of which quantity is to take over the rôle of the two dimensional central charge $c$, which in two dimensions turns up as the central charge of the conformal algebra, as the coefficient of the two point function of the energy-momentum tensor, and as the anomalous contribution to the trace of the energy-momentum tensor.

In the four dimensional trace anomaly, the following constants appear

$$
\left\langle T_{m}{ }^{m}\right\rangle=c C^{2}+a \tilde{\mathcal{R}}^{2}+b \mathcal{R}^{2}+f \square \mathcal{R},
$$

where $\mathcal{R}$ is the scalar curvature (Ricci scalar), $C^{2}$ is the square of the Weyl tensor, and $\tilde{\mathcal{R}}^{2}$ is the Euler density,

$$
\begin{aligned}
C^{2} & :=C_{a b c d} C^{a b c d}=\mathcal{R}^{a b c d} \mathcal{R}_{a b c d}-2 \mathcal{R}^{a b} \mathcal{R}_{a b}+\frac{1}{3} \mathcal{R}^{2} \\
\tilde{R}^{2} & :=\mathcal{R}^{a b c d} \mathcal{R}_{a b c d}-4 \mathcal{R}^{a b} \mathcal{R}_{a b}+\mathcal{R}^{2}
\end{aligned}
$$

There are known counter examples for a " $c$ "-theorem in four space-time dimensions but that still leaves open the possibility of an $a$-theorem [46], which holds in all examples that permit explicit checking. Since these are supersymmetric theories, it may well be that supersymmetry is a necessary ingredient for the irreversibility of RG flows. (As an aside in all known examples of holographic renormalisation group flows that permit determination of the anomaly coefficients on both ends of the flow it holds $c=a$. On the supergravity side monotonicity of the flow is related to energy conditions as they have to be employed in causality considerations in Einstein gravity [10].) Often by an abuse of language the $a$-theorem is also called $c$-theorem, even though the prefactor of Euler density is conventionally denoted " $a$ ". 


\subsection{Local RG Equation and the $c$-Theorem}

The analysis of this Section will be confined to idealised renormalisable field theories that are classically conformally invariant and involve a set of coupling constants $\lambda^{i}$ corresponding to local scalar operators $\mathcal{O}^{i}$. Due to conformal invariance the coupling constants should have mass dimension zero such that the operator's mass dimension should be equal to the spacetime dimension.

When the theory is not conformally invariant on the quantum level the trace of the energy-momentum tensor is non-vanishing and can be expressed in terms of some operator basis formed by $\mathcal{O}^{i}$

$$
\left\langle T_{m}{ }^{m}\right\rangle=\beta^{i}\left\langle\left[\mathcal{O}_{i}\right]\right\rangle
$$

where $\left[\mathcal{O}_{i}\right]$ denotes a (by some renormalisation scheme) well-defined operator insertion and $\beta^{i}$ are the beta functions associated to the corresponding couplings $\lambda^{i}$.

When Weyl symmetry is preserved during quantisation, the beta functions and therefore the trace of the energy-momentum tensor vanish.

Promoting the coupling constants $\lambda^{i}$ to fields as well as the metric,

$$
\begin{gathered}
\lambda^{i} \mapsto \lambda^{i}(x), \\
\eta_{m n} \mapsto g_{m n}(x),
\end{gathered}
$$

allows to give well-defined expressions for the operators $\mathcal{O}_{i}$ (the bracket indicating that the operator is well-defined will be silently dropped, henceforth) and the energy-momentum tensor,

$$
\mathcal{O}_{i}(x):=\frac{\delta}{\delta \lambda^{i}(x)} W, \quad T^{m n}(x):=2 \frac{\delta}{\delta g_{m n}(x)} W .
$$

This requires the theory to be defined for a general curved background metric $g_{m n}$. In addition to the counterterms present in the QFT on flat space with constant couplings, which give rise to the usual running of couplings, generically there should be now also counterterms $\mathcal{A}$ depending on the curvature and on $\partial_{m} \lambda^{i}$, which vanish in the limit of constant couplings and metric. In particular (7.26) acquires additional contributions 
according to

$$
\left\langle T_{m}{ }^{m}\right\rangle=\beta^{i}\left\langle\mathcal{O}_{i}\right\rangle+\nabla_{m}\left\langle\mathcal{J}^{m}\right\rangle+\mathcal{A}
$$

with $\mathcal{J}^{m}$ a local current. In general the trace above is not a local expression, which is why it was important to introduce space-time dependent couplings to give a meaning to any products of finite operators by functional derivatives with respect to couplings or the metric. The essential assumption is that the anomaly $\mathcal{A}$ stays a local expression to all orders, or in other words that the non-local contribution to the vacuum expectation value of the trace is contained in $\left\langle\mathcal{O}_{i}\right\rangle$.

The statement (7.30) can be recast in the form

$$
\Delta_{\sigma}^{W} W=\Delta_{\sigma}^{\beta} W-\int d^{D} x \sqrt{g} \mathcal{A}\left(\sigma, \mathcal{R}_{a b c d}, \partial_{m} \lambda^{i}\right)
$$

where $W=\ln \int[d \phi] \exp (-S / \hbar)$ is the generating functional of the connected Green's functions, $\sigma$ is the parameter of Weyl transformation generated by $\Delta_{\sigma}^{W}$ and

$$
\begin{aligned}
\Delta_{\sigma}^{W}:=2 \int d V g^{m n} \frac{\delta}{\delta g^{m n}}, & d V=d^{D} x \sqrt{g}, \\
\Delta_{\sigma}^{\beta} & :=\int d V \sigma \beta^{i} \frac{\delta}{\delta \lambda^{i}},
\end{aligned}
$$

with $D$ the number of space-time dimensions.

Equation (7.31) is in effect a local version of the (anomalous) CallanSymanzik equation

$$
\left[\mu \frac{\partial}{\partial \mu}+\beta^{i} \frac{\partial}{\partial \lambda^{i}}\right] W=\mathcal{A} .
$$

The shape of $\mathcal{A}\left(\sigma, \mathcal{R}_{a b c d}, \partial_{m} \lambda^{i}\right)$ is restricted by power counting and the requirement to vanish in the flat space/constant coupling limit, such that in this limit the local RG equation (7.31) reduces to the homogeneous Callan-Symanzik equation when imposing the condition

$$
\left[\mu \frac{\partial}{\partial \mu}+2 g^{m n} \frac{\delta}{\delta g^{m n}}\right] W=0,
$$


which is a consequence of naïve dimensional analysis.

As a simple example a possible parametrisation of the ambiguous* anomaly in two dimensions is

$$
\left(\Delta_{\sigma}^{W}-\Delta_{\sigma}^{\beta}\right) W=\int d V\left[\sigma\left(\frac{1}{2} c \mathcal{R}+\frac{1}{2} \chi_{i j} \partial_{m} \lambda^{i} \partial^{m} \lambda^{i}\right)+\left(\partial_{m} \sigma\right) w_{i} \partial^{m} \lambda^{i}\right]
$$

with $c, \chi_{i j}$ and $w_{i}$ arbitrary function of the couplings, which may be determined in a perturbative expansion with the assumption that the above shape is preserved to all orders, and partial derivatives $\partial_{i}:=\partial_{\lambda^{i}}$.

Wess-Zumino consistency

A further constraint on the anomaly with far less trivial consequences arises from Weyl transformations being Abelian, which implies

$$
\left[\Delta_{\sigma}^{W}-\Delta_{\sigma}^{\beta}, \Delta_{\sigma^{\prime}}^{W}-\Delta_{\sigma^{\prime}}^{\beta}\right]=0
$$

This Wess-Zumino consistency condition renders the determination of the trace anomaly an algebraic (cohomological) problem.

In the case of two dimensions (7.36) the consistency condition yields

$$
\begin{aligned}
& {\left[\Delta_{\sigma}^{W}-\Delta_{\sigma}^{\beta}, \Delta_{\sigma^{\prime}}^{W}-\Delta_{\sigma^{\prime}}^{\beta}\right]=\int d V\left(\sigma^{\prime} \partial_{m} \sigma-\sigma \partial_{m} \sigma^{\prime}\right) V^{m},} \\
& V_{m}=\left(\partial_{m} \lambda^{i}\right)\left(\partial_{i}\left(c+w_{j} \beta^{j}\right)-\chi_{i j} \beta^{j}+\left(\partial_{i} w_{j}-\partial_{j} w_{i}\right) \beta^{j}\right)
\end{aligned}
$$

and therefore the following coefficient consistency condition holds

$$
\beta^{i} \partial_{i}\left(c+w_{j} \beta^{j}\right)=\chi_{i j} \beta^{i} \beta^{j}
$$

The arbitrariness of $W$ with respect to local functionals of the fields

$$
\delta W=\int d V\left(\frac{1}{2} b \mathcal{R}-\frac{1}{2} c_{i j} \partial_{m} \lambda^{i} \partial^{m} \lambda^{j}\right)
$$

*In this formulation the anomaly is of course only determined up to partial integrations. Furthermore it is only defined up to adding local counterterms to the vacuum energy functional $W$. 
implies for the coefficients

$$
\begin{aligned}
\delta c & =\beta^{i} \partial_{i} b, & \delta \chi_{i j}=\mathcal{L}_{\beta} \chi_{i j} & =\beta^{k} \partial_{k} c_{i j}+2 \beta_{i} \beta^{k} c_{k j}, \\
\delta w_{i} & =-\partial_{i} b+c_{i j} \beta^{j}, & \delta\left(c+w_{j} \beta^{j}\right) & =c_{i j} \beta^{i} \beta^{j} .
\end{aligned}
$$

The Zamolodchikov metric $G_{i j}$,

Zamolodchikov

$$
G_{i j}(t)=\frac{1}{8}\left(x^{2}\right)^{2}\left\langle\mathcal{O}_{i}(x) \mathcal{O}_{j}(0)\right\rangle, \quad t=\frac{1}{2} \ln \mu^{2} x^{2},
$$

is positive by unitarity (or reflection positivity in Euclidean space). It can be shown that $G_{i j}=\chi_{i j}+\mathcal{L}_{\beta} c_{i j}$.

Then the function

$$
C:=3\left(c+w_{i} \beta^{i}+c_{i j} \beta^{i} \beta^{j}\right)
$$

is monotonic by (7.40) and positive definiteness of $G_{i j}$,

$$
C^{\prime}=-\beta^{i} \partial_{i} C=-3 G_{i j} \beta^{i} \beta^{j}<0 .
$$

This is Zamolodchikov's famous $c$-theorem.

Of course there is more to be said about renormalisation scheme dependence, for details see [48]. Here it shall suffice to mention that equation (7.40) is invariant under (7.41).

\subsection{1 $a$-Theorem}

The same calculation can be repeated in four space-time dimensions, giving rise to a system of coefficient consistency equations much more involved than the two dimensional example. The complete set of anomaly terms and consistency equations shall not be reproduced here, the interested reader is referred to [48] instead.

Omitting a number of less interesting terms, a sketch of the four di- 
mensional trace anomaly is given by

$$
\begin{aligned}
{\left[\Delta_{\sigma}^{W}-\Delta_{\sigma}^{\beta}\right] W=\int d V \sigma[a} & \tilde{\mathcal{R}}^{2}+c C^{2}+b \mathcal{R}^{2} \\
& +\frac{1}{2} \chi_{i j}^{g} \mathcal{G}^{m n} \partial_{m} \lambda^{i} \partial^{n} \lambda^{j}+\frac{1}{2} \chi_{i j}^{a} \nabla^{2} \lambda^{i} \nabla^{2} \lambda^{j} \\
& \left.+\frac{1}{2} \chi_{i j k}^{b} \partial_{m} \lambda^{i} \partial^{m} \lambda^{j} \nabla^{2} \lambda^{k}+\ldots\right]
\end{aligned}
$$

with $\tilde{\mathcal{R}}^{2}, C^{2}, \mathcal{R}^{2}, \mathcal{G}_{m n}$ the Euler density, square of the Weyl tensor and Ricci scalar and the Einstein tensor, respectively.

The coefficient consistency equation analogue to (7.40) reads

$$
\beta^{i} \partial_{i}\left(a+\frac{1}{8} w_{j} \beta^{j}\right)=\frac{1}{8} \chi_{i j}^{g} \beta^{i} \beta^{j}
$$

By virtue of a further consistency equation,

$$
\chi_{i j}^{g}+2 \chi_{i j}^{a}+2 \partial_{i} \beta^{k} \chi_{k j}^{a}+\beta^{k} \chi_{k i j}^{b}=\mathcal{L}_{\beta} S_{i j}
$$

where $-\chi_{i j}^{a}$ can be shown to be positive definite, there might be hope to find a four-dimensional "a-theorem", when getting under control the other coefficients $\chi_{k i j}^{b}$ and $S_{i j}$. In the bosonic sector discussed by Osborn, this seems not feasible. However there might be additional constraints in supersymmetric theories. This is the topic of the next Chapter. 
The most exciting phrase to hear in science, the one that heralds new discoveries, is not "Eureka!" but "That's funny ..."

Isaac Asimov

\section{Chapter 8}

\section{Supersymmetric Trace Anomaly}

$\S 8.1$ SUSY Local RG Equation, 135. §8.2 Basis for the Trace Anomaly, 137. §8.3 Wess-Zumino Consistency Conditions, 141. §8.4 Local Counterterms, 143. §8.5 S-duality, 144. $\S 8.6$ Towards a Proof, 146. $\S 8.7$ Superfield Riegert Operator, 147. §8.8 Discussion, 150.

This Chapter generalises the local renormalisation group equation reviewed in the previous Chapter to a minimal supergravity framework. A basis for the trace anomaly is found and the consequences of the Wess-Zumino consistency conditions for super-Weyl transformations are evaluated.

\subsection{SUSY Local RG Equation}

The (integrated) local Callan-Symanzik (CS) equation of the previous Chapter reads

$$
\begin{gathered}
{\left[\int d^{4} x \sqrt{-g} \sigma(x)\right.} \\
\left.2 g^{m n} \frac{\delta}{\delta g_{m n}}+\int d^{4} x \sqrt{-g} \sigma(x) \beta^{i} \frac{\delta}{\delta \lambda^{i}(x)}\right] W \\
=\int d^{4} x \sqrt{-g} A\left(\sigma, \lambda^{i}\right) .
\end{gathered}
$$


chiral coupling Generically the action for a supersymmetric Yang-Mills theory reads

$$
\begin{aligned}
S & =\frac{1}{8 \pi} \lambda \int d^{6} z \operatorname{Tr} W^{\alpha} W_{\alpha}+\text { c.c. }, \\
W^{\alpha} & =-\frac{1}{8} \bar{D}^{2}\left(\mathrm{e}^{-2 V} D_{\alpha} \mathrm{e}^{2 V}\right),
\end{aligned}
$$

with $\lambda$ the coupling constant, which may be complex,

$$
\lambda=\frac{4 \pi}{g^{2}}-\frac{i \theta}{2 \pi}
$$

Because the action is chiral it is natural to promote the complex couplings to chiral fields as well.

Coupling to minimal supergravity, which is both the simplest and best explored choice, implies that the Weyl parameter $\sigma(x)$ becomes a chiral field too. Furthermore the supersymmetric generalisation of the trace of the energy-momentum tensor ("supertrace") is also chiral and defined by

$$
\mathcal{T}=\varphi \frac{\delta S}{\delta \varphi}
$$

The supertrace is related to the supercurrent by

$$
\overline{\mathcal{D}}^{\dot{\alpha}} \mathcal{T}_{\alpha \dot{\alpha}}=-\frac{2}{3} \mathcal{D}_{\alpha} \mathcal{T}
$$

where the supercurrent is defined by

$$
\mathcal{T}_{\alpha \dot{\alpha}}=\frac{\delta S}{\delta \mathbf{H}_{\alpha \dot{\alpha}}},
$$

with $\mathbf{H}_{\alpha \dot{\alpha}}$ corresponding to the gravitational superfield.*

Accordingly a SUSY version of (8.1) should be given by [102]

$$
\left[\int d^{6} z \sigma \varphi \frac{\delta}{\delta \varphi}-\int d^{6} z \sigma \beta^{i} \frac{\delta}{\delta \lambda^{i}}+\text { c.c. }\right] W=A+\text { c.c., }
$$

where $A$ denotes the anomaly which consists entirely of terms that contain

${ }^{*}$ To be precise, it is the quantum superfield associated to the gravitational superfield $H_{\alpha \dot{\alpha}}$ in quantum-background splitting. In Wess-Zumino gauge the lowest component of the gravitational superfield $H_{\alpha \dot{\alpha}}$ contains the vierbein. 
supergravity fields or depend on a derivative of $\lambda$ or $\bar{\lambda}$,

$$
A=\int d^{6} z \phi^{3} \sigma \mathcal{A}
$$

Using the differential operators

$$
\begin{aligned}
\Delta_{\sigma, \bar{\sigma}}^{W} & :=\Delta^{W}+\bar{\Delta}^{W}, \\
\Delta_{\sigma, \bar{\sigma}}^{\beta} & :=\Delta^{\beta}+\bar{\Delta}^{\beta}, \\
\Delta^{W} & :=\int d^{6} z \sigma \phi \frac{\delta}{\delta \phi}, \\
\Delta^{\beta} & :=\int d^{6} z \sigma \beta \frac{\delta}{\delta \lambda},
\end{aligned}
$$

the SUSY local RG equation can be recast into the form

$$
\left(\Delta^{W}-\Delta^{\beta}\right) W=A+\bar{A}
$$

It is convenient to additionally split this local CS equation into a chiral local CS equation and anti-chiral equation,

$$
\begin{aligned}
& \left(\Delta^{W}-\Delta^{\beta}\right) W=A \\
& \left(\bar{\Delta}^{W}-\bar{\Delta}^{\beta}\right) W=\bar{A},
\end{aligned}
$$

which gives rise to the following two Wess-Zumino consistency conditions, Wess-Zumino

$$
\begin{gathered}
{\left[\Delta_{\sigma}^{W}-\Delta_{\sigma}^{\beta}, \Delta_{\sigma^{\prime}}^{W}-\Delta_{\sigma^{\prime}}^{\beta}\right] W=0,} \\
{\left[\Delta_{\bar{\sigma}}^{W}-\Delta_{\bar{\sigma}}^{\beta}, \Delta_{\sigma}^{W}-\Delta_{\sigma}^{\beta}\right] W=0 .}
\end{gathered}
$$

It remains to find a suitable expression for the anomaly $A$.

\subsection{Basis for the Trace Anomaly}

In this Section a basis of dimension two operators is constructed that consists strictly of supergravity superfields (supertorsions) and covariant chiral derivatives and furthermore contains no fields with negative powers.*

${ }^{*}$ Due to the peculiarities of curved superspace there is actually a seemingly nonlocal term namely $R^{-1} W_{\alpha \beta \gamma} W^{\alpha \beta \gamma}$, which is Weyl covariant by itself and could be 


\begin{tabular}{|c|c|c|c|}
\hline \multicolumn{4}{|c|}{ Supergravity Fields } \\
\hline quantity & dimension & undotted & dotted \\
\hline$R$ & 1 & 0 & 0 \\
\hline $\bar{R}$ & 1 & 0 & 0 \\
\hline $\mathcal{D}$ & $1 / 2$ & 1 & 0 \\
\hline$\overline{\mathcal{D}}$ & $1 / 2$ & 0 & 1 \\
\hline $\mathcal{D}$ & 1 & 1 & 1 \\
\hline$G \dot{\alpha}$ & 1 & 1 & 1 \\
\hline$G$ & $3 / 2$ & 3 & 0 \\
\hline$W$ & $3 / 2$ & 0 & 3 \\
\hline
\end{tabular}

Table 8.1: Dimensional Analysis for Supergravity Fields: The total dimension of any basis term has to be two, the number of respective dotted and undotted indices even.

By assumption (see Section 8.1) the Weyl parameter $\sigma$ and the couplings $\lambda^{i}$ are chiral scalar fields.

The strategy for finding a basis of dimension two operators is as follows.

1. Use the freedom to partially integrate to remove any derivatives on the Weyl parameter $\sigma$. The anomaly then has the shape

$$
\Delta^{W} \Gamma=\int d^{8} z E^{-1} \sigma \mathcal{B}(\lambda, \bar{\lambda}) \cdot \mathcal{A}
$$

with $\mathcal{A}=\mathcal{A}\left(R, \bar{R}, G_{\alpha \dot{\alpha}}, W_{\alpha \beta \gamma}, \bar{W}_{\dot{\alpha} \dot{\beta} \dot{\gamma}}, \mathcal{D}, \overline{\mathcal{D}}, \mathcal{D} \lambda, \overline{\mathcal{D}} \bar{\lambda}\right)$

2. Expand in derivatives on couplings. Since the overall scaling dimension is supposed to be two, there are at most four derivatives and consequently at most four couplings in $\mathcal{A}$.

Furthermore since all basis terms for $\mathcal{A}$ should be scalars, the total number of indices should be even (dotted and undotted indices respectively). The properties relevant to these simple counting arguments are summarised in Table 8.1.

trivially included in the discussion. The expression is related to the Pontryagin invariant. 
The following combinations (bars not yet included) have a chance to yield the right dimension and index structure:

$$
2 \times R, \quad 2 \times G, \quad(1 \times R, 2 \times \mathcal{D}), \quad(1 \times G, 2 \times \mathcal{D}), \quad 4 \times \mathcal{D} .
$$

Taking into account the algebra and Bianchi identities, several derivatives acting on the same coupling $\lambda$ can be brought to a standard order. I chose

$$
\mathcal{D}_{\alpha} \lambda, \quad \mathcal{D}^{2} \lambda, \quad \mathcal{D}_{\alpha \dot{\alpha}} \lambda, \quad \mathcal{D}_{\alpha \dot{\alpha}} \mathcal{D}_{\beta} \lambda, \quad \mathcal{D}_{\alpha \dot{\alpha}} \mathcal{D}^{2} \lambda, \quad \mathcal{D}_{\alpha \dot{\alpha}} \mathcal{D}^{\alpha \dot{\alpha}} \lambda,
$$

and accordingly for $\bar{\lambda}$.

In total there arise 38 terms, such that the basis ansatz for the anomaly reads

$$
\begin{aligned}
\mathcal{B} & +\mathcal{A} \\
& =b^{(A)} G_{\alpha \dot{\alpha}} G^{\alpha \dot{\alpha}}+b^{(B)} R \bar{R}+b^{(C)} R^{2}+b^{(\bar{C})} \bar{R}^{2} \\
& +b^{(D)}\left(\mathcal{D}^{2} R\right)+b^{(\bar{D})}\left(\overline{\mathcal{D}}^{2} \bar{R}\right) \\
& +b_{i}^{(E)} R \mathcal{D}^{2} \lambda^{i}+b_{\bar{\imath}}^{(\bar{E})} \bar{R} \overline{\mathcal{D}}^{2} \bar{\lambda}^{\bar{\imath}} \\
& +b_{\bar{\imath}}^{(F)} R \overline{\mathcal{D}}^{2} \bar{\lambda}^{\bar{\imath}}+b_{i}^{(\bar{F})} \bar{R} \mathcal{D}^{2} \lambda^{i}+b_{i}^{(G)}\left(\mathcal{D}^{\alpha} R\right)\left(\mathcal{D}_{\alpha} \lambda^{i}\right)+b_{\bar{\imath}}^{(\bar{G})}\left(\overline{\mathcal{D}}_{\dot{\alpha}} \bar{R}\right)\left(\overline{\mathcal{D}}^{\dot{\alpha}} \bar{\lambda}^{\bar{\imath}}\right) \\
& +b_{i}^{(H)} G^{\alpha \dot{\alpha}} \mathcal{D}_{\alpha \dot{\alpha}} \lambda^{i}+b_{\bar{\imath}}^{(\bar{H})} G^{\alpha \dot{\alpha}} \mathcal{D}_{\alpha \dot{\alpha}} \bar{\lambda}^{\bar{\imath}}+b_{i}^{(I)} \mathcal{D}^{\alpha \dot{\alpha}} \mathcal{D}_{\alpha \dot{\alpha}} \lambda^{i}+b_{\bar{\imath}}^{(\bar{I})} \mathcal{D}^{\alpha \dot{\alpha}} \mathcal{D}_{\alpha \dot{\alpha}} \bar{\lambda}^{\bar{\imath}} \\
& +b_{i j}^{(J)} R\left(\mathcal{D}^{\alpha} \lambda^{i}\right)\left(\mathcal{D}_{\alpha} \lambda^{j}\right)+b_{\bar{\imath} \bar{\jmath}}^{(\bar{J})} \bar{R}\left(\overline{\mathcal{D}}_{\dot{\alpha}} \bar{\lambda}^{\bar{\imath}}\right)\left(\overline{\mathcal{D}}^{\dot{\alpha}} \bar{\lambda}^{\bar{\jmath}}\right) \\
& +b_{i j}^{(K)} \bar{R}\left(\mathcal{D}^{\alpha} \lambda^{i}\right)\left(\mathcal{D}_{\alpha} \lambda^{j}\right)+b_{\bar{\imath} \bar{\jmath}}^{(\bar{K})} R\left(\overline{\mathcal{D}}_{\dot{\alpha}} \bar{\lambda}^{\bar{\imath}}\right)\left(\overline{\mathcal{D}}^{\dot{\alpha}} \bar{\lambda}^{\bar{\jmath}}\right) \\
& +b_{i \bar{\jmath}}^{(L)} G^{\alpha \dot{\alpha}}\left(\mathcal{D}_{\alpha} \lambda^{i}\right)\left(\overline{\mathcal{D}}_{\dot{\alpha}} \bar{\lambda}^{\bar{\jmath}}\right)+b_{i \bar{\jmath}}^{(M)}\left(\mathcal{D}^{\alpha \dot{\alpha}} \lambda^{i}\right)\left(\mathcal{D}_{\alpha \dot{\alpha}} \bar{\lambda}^{\bar{\jmath}}\right) \\
& +b_{i j}^{(N)}\left(\mathcal{D}^{\alpha \dot{\alpha}} \lambda^{i}\right)\left(\mathcal{D}_{\alpha \dot{\alpha}} \lambda^{j}\right)+b_{\bar{\imath} \bar{\jmath}}^{(\bar{N})}\left(\mathcal{D}^{\alpha \dot{\alpha}} \bar{\lambda}^{\bar{\jmath}}\right)\left(\mathcal{D}_{\alpha \dot{\alpha}} \bar{\lambda}^{\bar{\jmath}}\right) \\
& +b_{i \bar{\jmath}}^{(O)}\left(\mathcal{D}^{\alpha} \lambda^{i}\right)\left(\mathcal{D}_{\alpha \dot{\alpha}} \overline{\mathcal{D}}^{\dot{\alpha}} \bar{\lambda}^{\bar{\jmath}}\right)+b_{\bar{\imath} j}^{(\bar{O})}\left(\overline{\mathcal{D}}^{\dot{\alpha}} \bar{\lambda}^{\bar{\imath}}\right)\left(\mathcal{D}_{\alpha \dot{\alpha}} \mathcal{D}^{\alpha} \lambda^{j}\right) \\
& +b_{i \bar{\jmath}}^{(P)}\left(\mathcal{D}^{2} \lambda^{i}\right)\left(\overline{\mathcal{D}}^{2} \bar{\lambda}^{\bar{\jmath}}\right) \\
& +b_{i j}^{(Q)}\left(\mathcal{D}^{2} \lambda^{i}\right)\left(\mathcal{D}^{2} \lambda^{j}\right)+b_{\bar{\imath} \bar{\jmath}}^{(\bar{Q})}\left(\overline{\mathcal{D}}^{2} \bar{\lambda}^{\bar{\imath}}\right)\left(\overline{\mathcal{D}}^{2} \bar{\lambda}^{\bar{\jmath}}\right)
\end{aligned}
$$




$$
\begin{aligned}
& +b_{i j k}^{(R)}\left(\mathcal{D}^{\alpha} \lambda^{i}\right)\left(\mathcal{D}_{\alpha} \lambda^{j}\right)\left(\mathcal{D}^{2} \lambda^{k}\right)+b_{\bar{\imath} \overline{\bar{k}}}^{(\bar{R})}\left(\overline{\mathcal{D}}_{\dot{\alpha}} \bar{\lambda}^{\bar{\imath}}\right)\left(\overline{\mathcal{D}}^{\dot{\alpha}} \bar{\lambda}^{\bar{\jmath}}\right)\left(\overline{\mathcal{D}}^{2} \bar{\lambda}^{\bar{k}}\right) \\
& +b_{i j \bar{k}}^{(S)}\left(\mathcal{D}^{\alpha} \lambda^{i}\right)\left(\mathcal{D}_{\alpha} \lambda^{j}\right)\left(\overline{\mathcal{D}}^{2} \bar{\lambda}^{\bar{k}}\right)+b_{\bar{\jmath} \bar{k}}^{(\bar{S})}\left(\overline{\mathcal{D}}_{\dot{\alpha}} \bar{\lambda}^{\bar{\imath}}\right)\left(\overline{\mathcal{D}}^{\dot{\alpha}} \bar{\lambda}^{\bar{\jmath}}\right)\left(\mathcal{D}^{2} \lambda^{k}\right) \\
& +b_{i j \bar{k}}^{(T)}\left(\mathcal{D}_{\alpha \dot{\alpha}} \lambda^{i}\right)\left(\mathcal{D}^{\alpha} \lambda^{j}\right)\left(\overline{\mathcal{D}}^{\dot{\alpha}} \bar{\lambda}^{\bar{k}}\right)+b_{\bar{\imath} k}^{(\bar{T})}\left(\mathcal{D}_{\alpha \dot{\alpha}} \bar{\lambda}^{\bar{\imath}}\right)\left(\mathcal{D}^{\alpha} \lambda^{k}\right)\left(\overline{\mathcal{D}}^{\dot{\alpha}} \bar{\lambda}^{\bar{\jmath}}\right) \\
& +b_{i j \bar{k} \bar{l}}^{(U)}\left(\mathcal{D}^{\alpha} \lambda^{i}\right)\left(\mathcal{D}_{\alpha} \lambda^{j}\right)\left(\overline{\mathcal{D}}_{\dot{\beta}} \bar{\lambda}^{\bar{k}}\right)\left(\overline{\mathcal{D}}^{\dot{\beta}} \bar{\lambda}^{\bar{l}}\right) \\
& +b_{i j k l}^{(V)}\left(\mathcal{D}^{\alpha} \lambda^{i}\right)\left(\mathcal{D}_{\alpha} \lambda^{j}\right)\left(\mathcal{D}^{\beta} \lambda^{k}\right)\left(\mathcal{D}_{\beta} \lambda^{l}\right) \\
& +b_{\bar{\imath} \bar{k} \bar{l}}^{(\bar{V})}\left(\overline{\mathcal{D}}_{\dot{\alpha}} \bar{\lambda}^{\bar{\imath}}\right)\left(\overline{\mathcal{D}}^{\dot{\alpha}} \bar{\lambda}^{\bar{\jmath}}\right)\left(\overline{\mathcal{D}}_{\dot{\beta}} \bar{\lambda}^{\bar{k}}\right)\left(\overline{\mathcal{D}}^{\dot{\beta}} \bar{\lambda}^{\bar{l}}\right) .
\end{aligned}
$$

where $b^{(A \ldots \bar{V})}$ are potentially functions of $\lambda$ and $\bar{\lambda}$.* However, this choice is not minimal as it still allows for partial integration with respect to $\overline{\mathcal{D}}_{\dot{\alpha}}$ because the chiral field $\sigma$ ignores these. Single derivatives on $\bar{\lambda}$ cannot be removed by partial integration in general, since a derivative acting on the coefficient $b$ reproduces the same term again.

More precisely, due to

$$
\begin{aligned}
\int d^{8} z b_{\bar{\jmath}}\left(\overline{\mathcal{D}}^{\dot{\alpha}} \bar{\lambda}^{\bar{\jmath}}\right) \bar{X}_{\dot{\alpha}} & =\int d^{8} z\left[\tilde{b}_{\bar{\jmath}}+\left(\partial_{\bar{\jmath}} \tilde{b}_{\bar{\imath}}\right) \bar{\lambda}^{\bar{\imath}}\right]\left(\overline{\mathcal{D}}^{\dot{\alpha}} \bar{\lambda}^{\bar{\jmath}}\right) \bar{X}_{\dot{\alpha}} \\
& =-\int d^{8} z \tilde{b}_{\bar{\jmath}} \bar{\lambda}^{\bar{\jmath}}\left(\overline{\mathcal{D}}^{\dot{\alpha}} \bar{X}_{\dot{\alpha}}\right), \\
b_{\bar{\jmath}} & =\partial_{\bar{\jmath}}\left(\tilde{b}_{\bar{\imath}} \bar{\lambda}^{\bar{\imath}}\right)
\end{aligned}
$$

a basis term with a single derivative on $\bar{\lambda}$ can only be removed from the tentative basis if a $\tilde{b}$ obeying (8.22) exists; i.e. the integrability conditions $\partial_{\bar{\imath}} b_{\bar{\jmath}}=\partial_{\bar{\jmath}} b_{\bar{\imath}}$ are fulfilled. This is certainly not true in general, but for only one coupling or if the theory is invariant under arbitrary exchange of the coupling constants $\bar{\lambda}^{\bar{\imath}} \leftrightarrow \bar{\lambda}^{\bar{j}}$, the basis reduces further.

Apart from this complication, removable terms are those which either have an outer $\overline{\mathcal{D}}$ derivative (as opposed to one being hidden behind a $\mathcal{D}^{\alpha}$ ) or can be brought to that form by using the Bianchi identities and the supergravity algebra.

The above "basis" not being a minimal set of operators is not really a problem (except for creating a bit of extra work in the followings), since it will be possible to consistently set to zero the prefactors to such super-

* Note that $b^{(T)}$ and $b^{(\bar{T})}$ are the only coefficients which potentially can be asymmetric in two indices of the same type. As we will see later, the variations are symmetric, so consistency conditions can only give results for the respective symmetric part. 
fluous terms belatedly.

\subsection{Wess-Zumino Consistency Conditions}

It is now time to evaluate the Wess-Zumino consistency conditions

$$
\begin{gathered}
{\left[\Delta_{\sigma}^{W}-\Delta_{\sigma}^{\beta}, \Delta_{\sigma^{\prime}}^{W}-\Delta_{\sigma^{\prime}}^{\beta}\right] W=0,} \\
{\left[\Delta_{\bar{\sigma}}^{W}-\Delta_{\bar{\sigma}}^{\beta}, \Delta_{\sigma}^{W}-\Delta_{\sigma}^{\beta}\right] W=0 .}
\end{gathered}
$$

As shall be seen, all necessary expressions can be determined from

$$
\left(\Delta_{\sigma}^{W}-\Delta_{\sigma}^{\beta}\right)\left(\Delta_{\sigma^{\prime}}^{W}-\Delta_{\sigma^{\prime}}^{\beta}\right) W
$$

which requires to calculate the Weyl variation of all basis terms as well as to determine the expressions

$$
\Delta_{\sigma}^{W}\left(\Delta_{\sigma^{\prime}}^{W}-\Delta_{\sigma^{\prime}}^{\beta}\right) W, \quad \Delta_{\sigma}^{\beta}\left(\Delta_{\sigma^{\prime}}^{W}-\Delta_{\sigma^{\prime}}^{\beta}\right) W
$$

Since the calculation is straight-forward but tedious, the results have been banned to appendices B, C and D.

The general structure of $(8.25)$ is

$$
\begin{aligned}
\left(\Delta_{\sigma}^{W}-\Delta_{\sigma}^{\beta}\right)\left(\Delta_{\sigma^{\prime}}^{W}-\Delta_{\sigma^{\prime}}^{\beta}\right) W & \\
=\int d^{8} z E^{-1} \sigma^{\prime}\{ & \sigma \mathcal{F}_{0}+\left(\mathcal{D}^{\alpha} \sigma\right) \mathcal{F}_{\alpha}+\left(\mathcal{D}^{2} \sigma\right) \mathcal{F}_{2}+\left(\mathcal{D}^{\alpha \dot{\alpha}} \sigma\right) \mathcal{F}_{\alpha \dot{\alpha}} \\
& \left.+\left(\mathcal{D}_{\alpha \dot{\alpha}} \mathcal{D}^{\alpha} \sigma\right) \overline{\mathcal{F}}_{3}^{\dot{\alpha}}+\left(\mathcal{D}_{\alpha \dot{\alpha}} \mathcal{D}^{\alpha \dot{\alpha}} \sigma\right) \mathcal{F}_{4}\right\}
\end{aligned}
$$

where the coefficients $\mathcal{F}$ can be determined from the intermediate results in appendix B and are listed in appendix $\mathrm{C}$.

The naming scheme for the anomaly terms has been chosen such that the calculation of the Weyl consistency conditions only requires

$$
\Delta_{\sigma} \Delta_{\sigma^{\prime}} W
$$

to be computed by variation. The reader may convince himself that the other three operator combinations can be determined from the following 
simple set of rules.

$$
\begin{aligned}
\Delta_{\sigma^{\prime}} \Delta_{\sigma} W & =\left(\Delta_{\sigma} \Delta_{\sigma^{\prime}} W\right)^{\sigma \leftrightarrow \sigma^{\prime}} ; \\
\Delta_{\sigma} \bar{\Delta}_{\bar{\sigma}} W & =\left(\Delta_{\sigma} \Delta_{\sigma^{\prime}} W\right)^{\mathbf{\Lambda}}, \\
\left(b^{(x)}\right)^{\mathbf{\Delta}} & :=\bar{b}^{(\bar{x})}, \\
\left(\sigma^{\prime}\right)^{\mathbf{\Lambda}} & :=\bar{\sigma} \\
(\sigma)^{\mathbf{\Lambda}} & :=\sigma, \\
(\ldots)^{\mathbf{\Delta}} & :=(\ldots) ; \\
\bar{\Delta}_{\bar{\sigma}} \Delta_{\sigma^{\prime}} W & =\overline{\left(\Delta_{\sigma} \Delta_{\sigma^{\prime}} W\right)^{\mathbf{\Lambda}}},
\end{aligned}
$$

where $(\ldots)$ denotes anything that is not covered by explicit prior rules. Note that for the few real terms, it holds $b^{(\bar{x})}=b^{(x)}$.

So the $[\Delta, \Delta]$ Wess-Zumino consistency condition (8.23) is

$$
\begin{aligned}
{\left[\Delta_{\sigma}^{W}-\Delta_{\sigma}^{\beta}, \Delta_{\sigma^{\prime}}^{W}-\Delta_{\sigma^{\prime}}^{\beta}\right] W } & \\
=\int d^{8} z E^{-1}\left(\sigma^{\prime} \mathcal{D}^{\alpha} \sigma-\right. & \left.\sigma \mathcal{D}^{\alpha} \sigma^{\prime}\right)\left\{\mathcal{F}_{\alpha}-\mathcal{D}_{\alpha}\left(\mathcal{F}_{2}-\frac{i}{4} \overline{\mathcal{D}}_{\dot{\alpha}} \overline{\mathcal{F}}_{3}^{\dot{\alpha}}\right)\right. \\
& \left.+\frac{i}{2} \overline{\mathcal{D}}^{\dot{\alpha}}\left(\mathcal{F}_{\alpha \dot{\alpha}}-\mathcal{D}_{\alpha \dot{\alpha}} \mathcal{F}_{4}\right)+i G_{\alpha \dot{\alpha}} \overline{\mathcal{F}}_{3}^{\dot{\alpha}}\right\},
\end{aligned}
$$

while the $[\Delta, \bar{\Delta}]$ Wess-Zumino consistency condition (8.24) yields

$$
\begin{aligned}
{\left[\Delta_{\bar{\sigma}}^{W}\right.} & \left.-\Delta_{\bar{\sigma}}^{\beta}, \Delta_{\sigma}^{W}-\Delta_{\sigma}^{\beta}\right] W \\
& =\int d^{8} z E^{-1}\left[\sigma \bar{\sigma}(\mathrm{b})+\sigma\left(\mathcal{D}^{\alpha \dot{\alpha}} \bar{\sigma}\right)(\mathrm{c})+\left(\mathcal{D}_{\alpha \dot{\alpha}} \sigma\right)\left(\mathcal{D}^{\alpha \dot{\alpha}} \bar{\sigma}\right)(\mathrm{d})\right]
\end{aligned}
$$

with (b), (c) and (d) the respective left hand sides of 


$$
\begin{aligned}
\mathcal{F}_{\alpha}- & \mathcal{D}_{\alpha}\left(\mathcal{F}_{2}-\frac{i}{4} \overline{\mathcal{D}}_{\dot{\alpha}} \overline{\mathcal{F}}_{3}^{\dot{\alpha}}\right) \\
& +\frac{i}{2} \overline{\mathcal{D}}^{\dot{\alpha}}\left(\mathcal{F}_{\alpha \dot{\alpha}}-\mathcal{D}_{\alpha \dot{\alpha}} \mathcal{F}_{4}\right)+i G_{\alpha \dot{\alpha}} \overline{\mathcal{F}}_{3}^{\dot{\alpha}}=0 \\
\left\{\mathcal{F}_{0}-\right. & \left(\mathcal{D}^{\alpha} \mathcal{F}_{\alpha}\right)+\left(\mathcal{D}^{2} \mathcal{F}_{2}\right)-\frac{1}{2} \mathcal{D}^{\alpha \dot{\alpha}}\left(\mathcal{F}_{\alpha \dot{\alpha}}-\mathcal{D}_{\alpha \dot{\alpha}} \mathcal{F}_{4}-\mathcal{D}_{\alpha} \overline{\mathcal{F}}_{3 \dot{\alpha}}\right) \\
& \left.-2 i\left(\overline{\mathcal{D}}_{\dot{\alpha}} \bar{R}\right) \overline{\mathcal{F}}_{3}^{\dot{\alpha}}-2 i G^{\alpha \dot{\alpha}}\left(\mathcal{D}_{\alpha} \overline{\mathcal{F}}_{3 \dot{\alpha}}\right)\right\}^{\mathbf{\Lambda}}-\text { c.c. }=0 \\
\left\{\mathcal{F}_{\alpha \dot{\alpha}}\right. & \left.-\mathcal{D}_{\alpha \dot{\alpha}} \mathcal{F}_{4}-\mathcal{D}_{\alpha} \overline{\mathcal{F}}_{3 \dot{\alpha}}\right\}^{\mathbf{\Lambda}}+\text { c.c. }=0 \\
& \mathcal{F}_{4}^{\mathbf{\Lambda}}=\overline{\mathcal{F}}_{4}^{\mathbf{\Delta}}
\end{aligned}
$$

which constitute the full set of consistency conditions on the level of abbreviations $\mathcal{F}$. The complex conjugate of (8.35a) is an additional part of this system.

These coefficient consistency equations are the main result of this Part. Unfortunately expanded out they fill about three pages and have been put into Appendix D, therefore.

\subsection{Local Counterterms}

The vacuum energy functional $W$ is only determined up to the addition of local counter terms $\delta W$, a convenient choice for which is provided by the basis used for the anomaly, since it allows to reuse the results from the Wess-Zumino consistency condition:

$$
\begin{aligned}
W & \equiv W+\delta W, \\
\delta W & =\int d^{8} z E^{-1} \delta \mathcal{B} \cdot \mathcal{A},
\end{aligned}
$$

with $\delta \mathcal{B} \cdot \mathcal{A}$ analogous to (8.21). To fulfil the reality requirement $\delta W=$ $\overline{\delta W}$, it is necessary (and sufficient) to choose the coefficients $\delta b$ from $\delta \mathcal{B}$ according to $\delta \bar{b}^{(x)}=\delta b^{(\bar{x})}$ for any $x .^{*}$

*In particular for coefficients of the single, real terms $(A),(B),(L),(M),(P),(U)$, this amounts to taking $b^{(x)}=\bar{b}^{(x)}$. 
Realising that

$$
\begin{aligned}
& \Delta_{\sigma} W=\int d^{8} z E^{-1} \sigma \mathcal{B} \cdot \mathcal{A},
\end{aligned}
$$

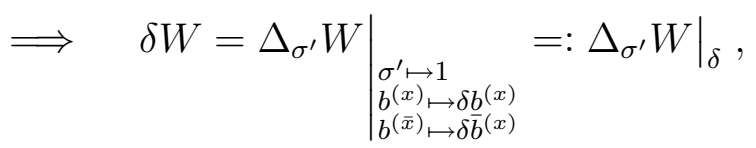

the effect of adding the local counter terms $\delta W$ to the generating functional $W$ is seen to be

$$
\begin{aligned}
\Delta_{\sigma}(W+\delta W)= & \Delta_{\sigma}\left(W+\left.\Delta_{\sigma^{\prime}} W\right|_{\delta}\right) \\
= & \int d^{8} z E^{-1} \sigma \mathcal{B} \cdot \mathcal{A} \\
& +\int d^{8} z E^{-1} \sigma\left\{\mathcal{F}_{0}-\mathcal{D}^{\alpha} \mathcal{F}_{\alpha}+\mathcal{D}^{2} \mathcal{F}_{2}-\mathcal{D}^{\alpha \dot{\alpha}} \mathcal{F}_{\alpha \dot{\alpha}}\right. \\
& \left.+\mathcal{D}^{\alpha} \mathcal{D}_{\alpha \dot{\alpha}} \overline{\mathcal{F}}_{3}^{\dot{\alpha}}+\mathcal{D}_{\alpha \dot{\alpha}} \mathcal{D}^{\alpha \dot{\alpha}} \mathcal{F}_{4}\right\}\left.\right|_{\delta},
\end{aligned}
$$

where in the last line equation (8.27) has been used.

In other words, the addition of local counter terms corresponds to the mapping

$$
\begin{aligned}
\mathcal{B} \cdot \mathcal{A} \mapsto \mathcal{B} \cdot \mathcal{A}+\left\{\mathcal{F}_{0}\right. & -\mathcal{D}^{\alpha} \mathcal{F}_{\alpha}+\mathcal{D}^{2} \mathcal{F}_{2}-\mathcal{D}^{\alpha \dot{\alpha}} \mathcal{F}_{\alpha \dot{\alpha}} \\
& \left.+\mathcal{D}^{\alpha} \mathcal{D}_{\alpha \dot{\alpha}} \overline{\mathcal{F}}_{3}^{\dot{\alpha}}+\mathcal{D}_{\alpha \dot{\alpha}} \mathcal{D}^{\alpha \dot{\alpha}} \mathcal{F}_{4}\right\}\left.\right|_{\delta}
\end{aligned}
$$

\subsection{S-duality}

$\mathcal{N}=4 \mathrm{SYM}$ is invariant under an $\mathrm{SL}(2, \mathbb{R})$ symmetry that is preserved on the quantum level. Explicit calculations indicate the symmetry is also maintained to one loop during coupling to gravity. Assuming that this is true to all orders, one might restrict the discussion of anomaly terms to superfield expressions that are manifestly invariant under that symmetry for the discussion of an $\mathcal{N}=4$ fixed point.

The theory of modular forms easily fills an entire book [103], but the consideration here shall be restricted to $\operatorname{SL}(2, \mathbb{R})$ invariant terms that can be build from the basis of anomaly terms (8.21).

In terms of the complex coupling $\lambda:=\frac{4 \pi}{g^{2}}-\frac{i \theta}{2 \pi}$, the $\mathrm{SL}(2, \mathbb{R})$ symmetry 
is generated by the two transformations

$$
\lambda \mapsto \frac{1}{\lambda}, \quad \lambda \mapsto \lambda+i,
$$

which have this unusual form due to employing the convention of taking the coupling constant $g^{-2}$ as the real part of $\lambda$.

It follows immediately that for coefficient functions $b(\lambda, \bar{\lambda})$ in the anomaly it holds $b=b(\lambda+\bar{\lambda})$.

In addition one observes

$$
\begin{aligned}
\frac{1}{\lambda+\bar{\lambda}} & \mapsto \lambda \bar{\lambda} \frac{1}{\lambda+\bar{\lambda}}, \\
\mathcal{D}_{\alpha} \lambda & \mapsto-\frac{1}{\lambda^{2}} \mathcal{D}_{\alpha} \lambda, \\
\overline{\mathcal{D}}_{\dot{\alpha}} \bar{\lambda} & \mapsto-\frac{1}{\bar{\lambda}^{2}} \overline{\mathcal{D}}_{\dot{\alpha}} \bar{\lambda}, \\
\mathbb{D}^{2} \lambda & \mapsto-\frac{1}{\lambda^{2}} \mathbb{D}^{2} \lambda, \\
\overline{\mathbb{D}}^{2} \bar{\lambda} & \mapsto-\frac{1}{\bar{\lambda}^{2}} \overline{\mathbb{D}}^{2} \bar{\lambda}, \\
\mathbb{D}_{\alpha \dot{\alpha}} \mathcal{D}^{\alpha} \lambda & \mapsto-\frac{1}{\lambda^{2}} \mathbb{D}_{\alpha \dot{\alpha}} \mathcal{D}^{\alpha} \lambda,
\end{aligned}
$$

where

$$
\begin{array}{r}
\mathbb{D}^{2} \lambda:=\mathcal{D}^{2} \lambda-\frac{2}{\lambda+\bar{\lambda}}\left(\mathcal{D}^{\alpha} \lambda\right)\left(\mathcal{D}_{\alpha} \lambda\right), \\
\overline{\mathbb{D}}^{2} \bar{\lambda}:=\overline{\mathbb{D}^{2} \lambda}=\overline{\mathcal{D}}^{2} \bar{\lambda}-\frac{2}{\lambda+\bar{\lambda}}\left(\overline{\mathcal{D}}_{\dot{\alpha}} \bar{\lambda}\right)\left(\overline{\mathcal{D}}^{\dot{\alpha}} \bar{\lambda}\right),
\end{array}
$$

Therefore S-invariant expressions are given by

$$
\begin{array}{ll}
\frac{1}{(\lambda+\bar{\lambda})^{2}}\left(\mathbb{D}^{2} \lambda\right)\left(\overline{\mathbb{D}}^{2} \bar{\lambda}\right), & \sim(P),(S),(\bar{S}),(U) \\
\frac{1}{(\lambda+\bar{\lambda})^{2}}\left(\mathcal{D}^{\alpha} \lambda\right)\left(\mathcal{D}_{\alpha} \overline{\mathbb{D}}^{2} \bar{\lambda}\right), & \sim(L),(O),(U),(\bar{T}) \\
\frac{1}{(\lambda+\bar{\lambda})^{2}}\left(\overline{\mathcal{D}}_{\dot{\alpha}} \mathbb{D}^{2} \lambda\right)\left(\overline{\mathcal{D}}^{\dot{\alpha}} \bar{\lambda}\right), & \\
\frac{1}{(\lambda+\bar{\lambda})^{2}}\left(\mathcal{D}_{\alpha \dot{\alpha}} \lambda\right)\left(\mathcal{D}^{\alpha \dot{\alpha}} \bar{\lambda}\right), & \sim(M) \\
\frac{1}{(\lambda+\bar{\lambda})^{2}} G^{\alpha \dot{\alpha}}\left(\mathcal{D}_{\alpha} \lambda\right)\left(\overline{\mathcal{D}}_{\dot{\alpha}} \bar{\lambda}\right), & \sim(L)
\end{array}
$$




$$
\frac{1}{(\lambda+\bar{\lambda})^{4}}\left(\mathcal{D}^{\alpha} \lambda\right)\left(\mathcal{D}_{\alpha} \lambda\right)\left(\overline{\mathcal{D}}_{\dot{\alpha}} \bar{\lambda}\right)\left(\overline{\mathcal{D}}^{\dot{\alpha}} \bar{\lambda}\right) \quad \sim(U)
$$

and moreover the $\lambda, \bar{\lambda}$ independent terms $(A)$ to $(\bar{D})$.

\subsection{Towards a Proof}

For the proof of Zamolodchikov's theorem in two dimensions, the crucial ingredient is the connection of the anomaly coefficients to correlation functions from which the positive definite Zamolodchikov metric was defined, see Sections 7.4 and 7.4.1 in particular.

As an example of how this procedure works the consistency condition (D.3f) from the appendix shall be discussed,

$$
-\frac{i}{2} b_{j \bar{k}}^{(M)}+\beta^{i} b_{j i \bar{k}}^{(T)}+i b_{j \bar{k}}^{(L)}+\frac{i}{2} b_{i j}^{(N)}\left(\partial_{\bar{k}} \beta^{i}\right)+\frac{i}{2} \beta^{i}\left(\partial_{\bar{k}} b_{i j}^{(N)}\right)-b_{i j \bar{k}}^{(T)} \beta^{i}=0 .
$$

$b_{i j \bar{k}}^{(T)}$ is the only coefficient function that is not (anti-)symmetric in indices of the same kind. From the expression above it can however be projected out by multiplying with $\beta^{j}$, which leaves

$$
\beta^{j}\left[b_{j \bar{k}}^{(M)}-2 b_{j \bar{k}}^{(L)}-\partial_{\bar{k}}\left(\beta^{i} b_{i j}^{(N)}\right)\right]=0,
$$

In fact $b_{i j}^{(N)}$ vanishes identically as a consequence of the RG equation, which for the anomaly restricted to that coefficient reads

$$
\mu \frac{\partial}{\partial \mu} W+\beta^{i} \partial_{i} W=b_{i j}^{(N)}\left(\mathcal{D}^{2} \lambda^{i}\right)\left(\mathcal{D}^{2} \lambda^{j}\right)
$$

Acting on it with $\frac{\delta}{\delta \lambda^{k}} \frac{\delta}{\delta \lambda^{l}}$, gives

$$
\mu \frac{\partial}{\partial \mu}\left\langle\mathcal{O}^{k} \mathcal{O}^{l}\right\rangle+\beta^{i} \partial_{i}\left\langle\mathcal{O}^{k} \mathcal{O}^{l}\right\rangle=b_{k l}^{(N)}\left(\mathcal{D}^{2} \delta^{6}(z)\right)\left(\mathcal{D}^{2} \delta^{6}\left(z^{\prime}\right)\right)
$$

where the left-hand side vanishes by non-renormalisation of chiral correlation functions. It immediately follows that $b_{i j}^{(N)} \equiv 0$, which means that equation (8.58) implies

$$
\beta^{j} \bar{\beta}^{\bar{k}}\left[b_{j \bar{k}}^{(M)}-2 b_{j \bar{k}}^{(L)}\right]=0 .
$$


This is the supersymmetric version of equation (7.49), which reads

$$
\chi_{i j}^{g}-2 \chi_{i j}^{a}=\mathcal{L}_{\beta} S_{i j}-2 \partial_{i} \beta^{k} \chi_{k j}^{a}-\beta^{k} \chi_{k i j}^{b},
$$

though from (8.61) the right hand side is zero when taking into account

$$
b^{(M)} \sim \chi^{g}-\chi^{a}, \quad b^{(L)} \sim \chi^{g},
$$

as will be seen from the component expansions (8.68)-(8.70) of the next Section. This is just as required for a proof of the $a$-theorem, since $\chi^{(a)}$ can be shown to be positive definite in a particular scheme. In that scheme,

$$
-\widehat{\chi^{a}}=\frac{x^{8} S_{4}}{192}\left\langle\mathcal{O}_{i}(x) \mathcal{O}_{j}(0)\right\rangle
$$

where the right hand side is positive definite by unitarity. The set of counterterms which are needed to change to a scheme where $\chi^{a}=\widehat{\chi^{a}}$ were determined in [104].

Of course the other anomaly terms might contribute further terms to the simple identification between $b^{(M)}, b^{(L)}$ and $\chi^{a}, \chi^{g}$, thus spoiling the success. Actually from the whole basis for the anomaly, there is only one term which could do so, namely $\left(\mathcal{D}^{2} \lambda\right)\left(\overline{\mathcal{D}}^{2} \bar{\lambda}\right)$, which seems harmless since its component expansion yields only $\left(\nabla^{2} \lambda\right)\left(\nabla^{2} \lambda^{*}\right)$. Moreover it is expected to conspire with the $(M)$ and $(L)$ terms from the anomaly basis to form a supersymmetric version of the "Riegert operator" as shall be explained now.

\section{7 $\quad$ Superfield Riegert Operator}

For $\mathcal{N}=4$ Yang-Mills theory [49] obtains a one-loop trace anomaly that contains the operator

$$
\frac{1}{\left(\lambda+\lambda^{*}\right)^{2}}\left(\nabla^{2} \lambda \nabla^{2} \lambda^{*}-2 \mathcal{G}^{m n} \nabla_{m} \lambda \nabla_{n} \lambda^{*}-\frac{1}{3} \mathcal{R} \nabla^{m} \lambda \nabla_{m} \lambda^{*}\right)
$$


which basically is the Riegert operator (7.17).* Note that the bosonic Riegert operator is a direct consequence of the (bosonic) consistency conditions for the $\mathcal{N}=4$ case. It is therefore important to reproduce the Riegert operator in the component expansion of the superfield formulation employed here.

This result indicates an inconsistency with our result because there does not seems to exist a superfield expression that generates this Riegert operator in a component expansion. Therefore it cannot be generated as part of the derived superfield trace anomaly.

component version

Strange enough in components a super-Weyl covariant version of this operator is known such that the following expression [51] is invariant under super-Weyl transformations,

$$
\begin{aligned}
\mathscr{L}= & e^{-1} \nabla^{2} \phi^{*} \nabla^{2} \phi-2\left(\mathcal{R}_{m n}-\frac{1}{3} g_{m n} \mathcal{R}\right) \nabla_{m} \phi^{*} \nabla_{n} \phi \\
& -\frac{1}{2} \bar{\chi}\left[\not D^{3}+\left(\mathcal{R}_{m n}-\frac{1}{6} g_{m n} \mathcal{R}\right) \gamma_{m} D_{n}\right] \chi \\
& -\frac{3}{4} \bar{\chi} \gamma_{m} D_{n} \chi F_{m n}+F^{*}\left[D^{2}-\frac{1}{6}\left(R-\bar{\psi}_{m} \mathcal{R}_{m}\right)\right] F \\
& + \text { (gravitino terms) }
\end{aligned}
$$

with

$$
D_{m} \chi=\nabla_{m} \chi+\frac{3 i}{4} \gamma_{5} A_{m} \chi, \quad D_{m}=\left(\partial_{m}+\frac{3 i}{2} A_{m}\right) F
$$

and $\phi, \psi, F$ the components of a chiral field of Weyl weight 0 .

Therefore one should expect a superfield version $\Delta_{R}^{4}$ of this operator to exist such that

$$
\delta_{\text {Weyl }}\left[\int d^{8} z E^{-1} \lambda \Delta_{R}^{4} \bar{\lambda}\right]=0
$$

with $\delta_{\text {Weyl }}$ indicating a super-Weyl transformation.

On the other hand one might simply use a component expansion of all expansion basis terms and determine the linear combination that yields the bosonic Riegert operator (7.17) as its lowest component.

Such a component expansion can be quite involved, but fortunately there is only a limit number of terms that can contribute. Here the dis-

*The factor in front plus some further terms are required to make the operator $\mathrm{SL}(2, \mathbb{R})$ invariant in addition. 
cussion shall be restricted to a few natural candidate terms which already produces some interesting results.

$$
\begin{aligned}
& \left.\mathcal{D}^{2}\left(\overline{\mathcal{D}}^{2}-4 R\right)\left(\mathcal{D}^{2} \lambda\right)\left(\overline{\mathcal{D}}^{2} \bar{\lambda}\right)\right|_{\mathrm{b}}=256\left(\nabla^{2} \lambda\right)\left(\nabla^{2} \lambda^{*}\right) \\
& \left.\mathcal{D}^{2}\left(\overline{\mathcal{D}}^{2}-4 R\right) G^{\alpha \dot{\alpha}}\left(\mathcal{D}_{\alpha} \lambda\right)\left(\overline{\mathcal{D}}_{\dot{\alpha}} \bar{\lambda}\right)\right|_{\mathrm{b}} \\
& \left.\quad=64\left(\mathcal{G}_{(\mu \nu)}+\frac{1}{4} g_{\mu \nu} \mathcal{R}\right)\left(\nabla^{\mu} \lambda\right)\left(\nabla^{\nu} \lambda^{*}\right)-\frac{16}{3} \mathcal{R}\left(\nabla^{\mu} \lambda\right)\left(\nabla_{\mu} \lambda^{*}\right)+\text { (imag. }\right) \\
& \left.\mathcal{D}^{2}\left(\overline{\mathcal{D}}^{2}-4 R\right)\left(\mathcal{D}_{\alpha \dot{\alpha}} \lambda\right)\left(\mathcal{D}^{\alpha \dot{\alpha}} \bar{\lambda}\right)\right|_{\mathrm{b}} \\
& =\frac{32}{3} \mathcal{R} g_{\mu \nu}\left(\nabla^{\mu} \lambda\right)\left(\nabla^{\nu} \lambda^{*}\right)-32\left(\nabla_{\mu} \lambda\right)\left(\nabla^{2} \nabla^{\mu} \lambda^{*}\right)+(\text { imag. }) \\
& =\frac{32}{3} \mathcal{R} g_{\mu \nu}\left(\nabla^{\mu} \lambda\right)\left(\nabla^{\nu} \lambda^{*}\right)-32 \mathcal{R}_{\mu \nu}\left(\nabla^{\mu} \lambda\right)\left(\nabla^{\nu} \lambda^{*}\right) \\
& \quad+32\left(\nabla^{2} \lambda\right)\left(\nabla^{2} \lambda^{*}\right)+(\text { total deriv. })
\end{aligned}
$$

where the following relations have been used,

$$
\begin{aligned}
{\left[\nabla_{\mu}, \nabla_{\nu}\right] V^{\rho} } & =\mathcal{R}_{\sigma \mu \nu}^{\rho} V^{\sigma} \\
\nabla^{2} \nabla_{\mu} V & =\nabla_{\mu} \nabla^{2} V+\mathcal{R}_{\nu \mu} \nabla^{\nu} V .
\end{aligned}
$$

First of all one should note that (8.68) can be expressed by a linear combination of (8.69) and (8.70) and a total derivative, which is just the component version of (6.93),

$$
\begin{aligned}
\left(\mathcal{D}^{2} \lambda\right)\left(\overline{\mathcal{D}}^{2} \bar{\lambda}\right)= & 4 G_{\alpha \dot{\alpha}}\left(\mathcal{D}^{\alpha} \lambda\right)\left(\overline{\mathcal{D}}^{\dot{\alpha}} \bar{\lambda}\right)+8\left(\mathcal{D}_{\alpha \dot{\alpha}} \lambda\right)\left(\mathcal{D}^{\alpha \dot{\alpha}} \bar{\lambda}\right) \\
& +(\text { total derivative })
\end{aligned}
$$

This relation being preserved in the component expansion is a strong indication for equations (8.68)-(8.70) to be correct.

Up to this identity the only combination of the candidate terms (8.68)(8.70) that yields the bosonic Riegert operator as its lowest component is

$$
\left(\mathcal{D}^{2} \lambda\right)\left(\overline{\mathcal{D}}^{2} \bar{\lambda}\right)-8 G_{\alpha \dot{\alpha}}\left(\mathcal{D}^{\alpha} \lambda\right)\left(\overline{\mathcal{D}}^{\dot{\alpha}} \bar{\lambda}\right)
$$

This combination is not super-Weyl covariant however and it turns out that for the anomaly basis (8.21), there is no non-trivial super-Weyl invariant expression that includes $\left(\mathcal{D}^{2} \lambda\right)\left(\overline{\mathcal{D}}^{2} \bar{\lambda}\right)$ - or $\left(\mathcal{D}_{\alpha \dot{\alpha}} \lambda\right)\left(\mathcal{D}^{\alpha \dot{\alpha}} \bar{\lambda}\right)$ by $(8.73)$. In other words, there is no superfield version of the Riegert operator for chiral fields of Weyl weight 0 . This is rather puzzling since the component 
version does exist. What may have gone wrong?

\subsection{Discussion}

Equation (8.73) provides a rather non-trivial consistency check for the component expansion and the Weyl variations are simple to check. One should therefore be confident that the result of the previous Section is correct.

minimal SUGRA Since the Weyl parameter in minimal supergravity is a chiral field, it naturally also encodes superlocal $\mathrm{U}(1)_{R}$ transformations. So perhaps one is simply requiring too much symmetry. Since the expressions are global $\mathrm{U}(1)_{R}$ invariant anyway, neglecting the local symmetry corresponds to allowing terms that contain derivatives acting on $\sigma-\bar{\sigma}$. Due to $\mathcal{D}_{\alpha}(\sigma-\bar{\sigma})=$ $\mathcal{D}_{\alpha}(\sigma+\bar{\sigma})$ this cannot be distinguished from super-Weyl transformations.

non-minimal

SUGRA
In non-minimal supergravity it is possible to not require invariance under local $\mathrm{U}(1)_{R}$, and a possible super-Weyl covariant operator (in the conventions of [98]) is given by

$$
\left(\mathbb{D}_{\alpha \dot{\alpha}} \lambda\right)\left(\mathbb{D}^{\alpha \dot{\alpha}} \bar{\lambda}\right)
$$

with the Weyl covariant vector derivative for scalar chiral yields of $\mathrm{U}(1)_{R}$ charge $y$ given by

$$
\begin{aligned}
\mathbb{D}_{\alpha \dot{\alpha}} & :=i\left(\bar{\nabla}_{\dot{\alpha}}-i\left(\frac{2}{3}+y\right) \bar{\Gamma}_{\dot{\alpha}}\right)\left(\nabla_{\alpha}+i y \Gamma_{\alpha}\right), \\
\delta\left[\mathbb{D}_{\alpha \dot{\alpha}} \lambda\right] & =L \mathbb{D}_{\alpha \dot{\alpha}} \lambda
\end{aligned}
$$

new-minimal SUGRA

In new-minimal supergravity the $\mathrm{U}(1)_{R}$ drops from the formulation and it is possible to give a superfield Riegert operator for linear superfields of Weyl-weight 0 that is covariant under the full invariance group of the supergravity algebra [52]

$$
\mathbf{D}_{\alpha \dot{\alpha}} \mathbf{D}^{\alpha \dot{\alpha}}+\frac{i}{3}\left(\mathcal{D}_{\alpha} \bar{T}_{\dot{\alpha}}+\overline{\mathcal{D}}_{\dot{\alpha}} T_{\alpha}\right) \mathbf{D}^{\alpha \dot{\alpha}}
$$

where $\mathbf{D}_{\alpha \dot{\alpha}}=\mathcal{D}_{\alpha \dot{\alpha}}-\frac{i}{12}\left(T_{\alpha} \overline{\mathcal{D}}_{\dot{\alpha}}+\bar{T}_{\dot{\alpha}} \mathcal{D}_{\alpha}\right)$ is a super-Weyl covariant derivative.

The difficulties to formulate fields of arbitrary Weyl and $\mathrm{U}(1)_{R}$ weight in a superconformal framework are long known (see for example [105]) and 
led to the introduction of a chiral compensating field. This can be most easily illustrated taking a chiral field $\lambda$ as an example. It clearly should transform under generalised super-Weyl transformations according to

$$
\lambda \mapsto \mathrm{e}^{n_{+} \sigma+n_{-} \bar{\sigma}} \lambda
$$

with $n_{+}$a real number and $n_{-}=0$ in order to stay a chiral field. In other words the type of the field dictates a fixed relation between its $\mathrm{U}(1)_{R}$ charge and its Weyl weight. Therefore a single field transforming as $\Phi \mapsto \mathrm{e}^{\sigma} \Phi$ can be used to bring all other fields to a fixed Weyl and U(1) weight, by redefinitions of the type $\tilde{\lambda}=\Phi^{-n_{+}} \lambda$ for example.

A suitable set of invariant supergravity fields is given by

Weyl invariant algebra

$$
\begin{array}{rlr}
\mathbb{D}_{\alpha}= & \mathbb{U} \mathcal{D}_{\alpha}-2\left(\mathcal{D}^{\beta} \mathbb{U}\right) M_{\alpha \beta}, & \\
\overline{\mathbb{D}}_{\dot{\alpha}}= & \overline{\mathbb{U}}_{\dot{\mathcal{D}}}-2(\overline{\mathcal{D}} \dot{\beta} \overline{\mathbb{U}}) \bar{M}_{\dot{\alpha} \dot{\beta}}, & \\
\mathbb{D}_{\alpha \dot{\alpha}}= & \frac{i}{2}\left\{\mathbb{D}_{\alpha}, \overline{\mathbb{D}}_{\dot{\alpha}}\right\}, & \\
\mathbb{T}_{\alpha}= & \mathbb{D}_{\alpha} \mathbb{T}, & \mathbb{T}=\ln \mathbb{U}^{4} \overline{\mathbb{U}}^{2}, \\
\mathbb{R}= & -\frac{1}{4}\left(\overline{\mathcal{D}}^{2}-4 R\right) \overline{\mathbb{U}}^{2}, & \mathbb{W}_{\alpha \beta \gamma}=\overline{\mathbb{U}}^{2} \mathbb{U} W_{\alpha \beta \gamma}, \\
\mathbb{G}_{\alpha \dot{\alpha}}= & \overline{\mathbb{U}} \mathbb{U} G_{\alpha \dot{\alpha}}+\frac{1}{2}\left(\overline{\mathbb{D}}_{\dot{\alpha}} \ln \mathbb{U}\right)\left(\mathbb{D}_{\alpha} \ln \mathbb{U}\right) \\
& +\frac{1}{4} \overline{\mathbb{D}}_{\dot{\alpha}} \mathbb{D}_{\alpha} \ln \left(\mathbb{U}^{2} \overline{\mathbb{U}}^{-1}\right)-\frac{1}{4} \mathbb{D}_{\alpha} \overline{\mathbb{D}}_{\dot{\alpha}} \ln \left(\overline{\mathbb{U}}^{2} \mathbb{U}^{-1}\right),
\end{array}
$$

where $\Psi$ is a linear conformal compensator which transforms under Weyl transformation $\varphi \mapsto \mathrm{e}^{\sigma} \varphi$ according to

$$
\Psi \mapsto \Psi^{\prime}=\exp \left[\frac{3 n-1}{3 n+1} \sigma-\bar{\sigma}\right] \Psi, \quad \overline{\mathcal{D}}_{\dot{\alpha}} \sigma=0
$$

The case $n=\frac{1}{3}$ corresponds to minimal supergravity and the compensator $\Phi:=\bar{\Psi}$ is a chiral field.

It should be remarked that the expressions (8.80) can be easily obtained by replacing $\sigma$ and $\bar{\sigma}$ in the Weyl transformed objects by $-\ln \Phi$ and $-\ln \bar{\Phi}$ in a similar way as in the bosonic case in Section 7.1.4.

One might think of taking the already known chiral compensator $\varphi^{-1}$ which as the compensator $\Phi$ in (8.80). However this use of the chiral compen- compensator sator $\varphi$, which is also a prepotential that transforms under the $\Lambda$ super- 
group, would break invariance under that symmetry. Another interesting possibility is the use of

$$
\Omega=1+\int d^{8} z^{\prime} E^{-1}\left(z^{\prime}\right) G_{+-}\left(z, z^{\prime}\right)
$$

where $G_{+-}$is the Feynman superpropagator defined by

$$
\frac{1}{4}\left(\mathcal{D}^{2}-4 \bar{R}\right)_{z} G_{+-}\left(z, z^{\prime}\right)=\delta^{6}\left(z, z^{\prime}\right)
$$

and $\delta^{6}\left(z, z^{\prime}\right)$ is the chiral delta distribution.

A simple consequence of the defining relation is

$$
\overline{\mathcal{D}}_{\dot{\alpha}} \Omega=0, \quad\left(\mathcal{D}^{2}-4 \bar{R}\right) \Omega=0,
$$

which implies $\Omega \mapsto \mathrm{e}^{-\sigma} \Omega$ under super-Weyl transformation and $\Omega$ is a suitable (though non-local) compensator. For superconformal backgrounds $\Omega$ actually becomes local and take the form

$$
\Omega=\varphi^{-1}+O(\mathscr{H})
$$

trivially Weyl With such a compensator the expression

invariant

$$
\left(\mathbb{D}^{2} \lambda\right)\left(\overline{\mathbb{D}}^{2} \bar{\lambda}\right)-8 \mathbb{G}_{\alpha \dot{\alpha}}\left(\mathbb{D}^{\alpha} \lambda\right)\left(\overline{\mathbb{D}}^{\dot{\alpha}} \bar{\lambda}\right)
$$

yields the bosonic Riegert operator and is super-Weyl invariant. Unfortunately the latter is also true for any other expression, so not much has been gained. In particular in the presence of a compensator the criterion for Weyl invariance of a term is the absence of any functional dependence on that compensating field, which is certainly not true for (8.86).

Another approach may be to ask what is a natural Weyl invariant operator for an arbitrary field, such that the operator does not coincide linear superfield with the Riegert operator. For example

$$
E^{-1}\left[\left(\mathcal{D}^{2}-4 \bar{R}\right) \psi\right]\left[\left(\overline{\mathcal{D}}^{2}-4 R\right) \bar{\psi}\right]
$$

is invariant when $\psi \mapsto \mathrm{e}^{\bar{\sigma}-\sigma} \psi$. This transformational behaviour is incompatible with $\psi$ being a chiral field. It is possible for $\psi$ being linear, but 
that assumption annihilates the operator of course.

For a real field $V$, a Weyl invariant operator is given by

real superfield

$$
E^{-1} V \mathcal{D}^{\alpha}\left(\overline{\mathcal{D}}^{2}-4 R\right) \mathcal{D}_{\alpha} V \equiv E^{-1} V \overline{\mathcal{D}}_{\dot{\alpha}}\left(\mathcal{D}^{2}-4 \bar{R}\right) \overline{\mathcal{D}}^{\dot{\alpha}} V
$$

with additional gauge invariance $V \mapsto V+\lambda+\bar{\lambda}$, where $\lambda$ and $\bar{\lambda}$ are chiral and anti-chiral fields respectively.

Since the $\mathcal{N}=4$ case should also incorporate $\operatorname{SL}(2, \mathbb{R})$ symmetry with invariance of the anomaly under

$$
\lambda \mapsto \lambda+i, \quad \lambda \mapsto \frac{1}{\lambda},
$$

one might be tempted to use the $\operatorname{SL}(2, \mathbb{R})$ Kähler form

$$
V=\ln \lambda+\bar{\lambda}
$$

to also include that symmetry. Of course the operator will then contain additional pieces acting on more than two fields. However those pieces which do act on only two fields form exactly the combination (8.73), such that the Riegert operator is missing again.

It seems that there is something in the minimal supergravity formalism that does not allow for superfield formulation of the Riegert operator. I strongly suspect that it is the $\mathrm{U}(1)_{R}$ symmetry that spoils the formulation of the operator by being inevitable tied to the super-Weyl transformations. 

When your work speaks for itself, don't interrupt.

Henry J. Kaiser

\section{Conclusions}

For the understanding of quantum field theories, its coupling to gravity backgrounds has proved a valuable tool. The discovery of AdS/CFT correspondence, which realises such a coupling holographically, has revived the interest in this idea and been a major break-through in the understanding of strongly coupled Yang-Mills theories. While the original AdS/CFT duality involves $\mathcal{N}=4$ supersymmetric Yang-Mills theory, it has soon been extended to less symmetric, more realistic theories.

In this work, such an extension is explored in more detail, taking as a extension of starting point the $\mathcal{N}=2$ supersymmetric D3/probe D7-brane framework AdS/CFT of [20], which is dual to $\mathcal{N}=4$ supersymmetric, large $N_{c} \mathrm{SU}\left(N_{c}\right)$ YangMills theory augmented by a small number $N_{f}$ of $\mathcal{N}=2$ hypermultiplets in the fundamental representation. By holographic methods, this theory's meson spectrum can be calculated analytically at quadratic order [24].

I considered first a geometry more general than the conventional $\mathrm{AdS}_{5} \times \mathrm{S}^{5}$ and second an instanton gauge configuration on the D7-branes. The general strategy was to introduce background configurations that reproduce the conventional setting in certain limits. This allowed to make contact with the ordinary AdS/CFT dictionary and is an important feature of this approach compared to others in the area that is sometimes referred to as AdS/QCD.

The following results were obtained:

- A holographic dual of spontaneous chiral symmetry breaking by a chiral symmetry breaking 
quark condensate

Goldstone boson

instantons on the

D7 bilinear quark condensate $\langle\bar{\psi} \psi\rangle$ was found. Since such a condensate is prohibited by supersymmetry, this required to use a background* that completely breaks supersymmetry and approximates $\mathrm{AdS}_{5} \times \mathrm{S}^{5}$ only towards the boundary. By standard AdS/CFT, the boundary of the space-time is associated to the ultraviolet of the dual field theory, such that the configuration describes an $\mathcal{N}=2$ theory that is relevantly deformed and flows to a non-supersymmetric infrared.

I calculated the quark condensate $\langle\bar{\psi} \psi\rangle$ as a function of the quark mass $m_{q}$, which gave a non-vanishing quark condensate in the limit $m_{q} \rightarrow 0$; i.e. sponetaneous chiral symmetry breaking. Moreover I determined the meson spectrum and demonstrated that the meson mode associated to the $\mathrm{U}(1)_{A}$ axial symmetry, which is geometrically realised as rotations, becomes massless in the $m_{q} \rightarrow 0$ limit as expected for a true Goldstone boson. When $m_{q} \neq 0$ this mode becomes a pseudo-Goldstone mode, which obeys the Gell-MannOakes-Renner relation $M_{\pi}^{2} \sim m_{q}$. In the large quark mass limit, the mesons lie in the supersymmetric regime such that their mass is degenerate and approximates the analytic results of the $\mathcal{N}=2$ theory.

In addition I determined the mass of highly excited scalar and pseudoscalar mesons, which have the interesting feature of not being degenerate in this setup.

- The dual description of the mixed Coulomb-Higgs branch of the $\mathcal{N}=2$ theory was found. The Higgs VEV corresponds to the size of an instanton configuration on the supergravity side, establishing a link between supersymmetry and the ADHM construction that was known to exist. Such an instanton configuration can only exist when there are at least two flavours, such that a non-Abelian Dirac-BornInfeld action had to be used. Ordering ambiguities can be avoided since a calculation to quadratic order is sufficient, but a crucial insight was the use of a singular gauge transformation to obtain the correct boundary behaviour consistent with the AdS/CFT dictionary. Having overcome this major obstacle, I numerically determined the

${ }^{*}$ Here a background by Gubser [38] was chosen. 
meson spectrum and found it to approach the analytic $\mathcal{N}=2$ spectrum in the limit of vanishing and infinite Higgs VEV, though in the latter case a non-trivial rearrangement was observed, which could be explained to arise from above singular gauge transformation.

- A geometric realisation of heavy-light mesons was developed; i.e. heavy-light mesons build up from a light and heavy quark providing a frame- meson work for the description of B mesons not available before. Since a realisation in terms of a non-Abelian D7-brane action only makes sense for small mass differences, a different approach has to be chosen. The configuration under consideration is that of a long string stretched between two D7-branes with a large separation, where the D7-branes are arranged to correspond to a massless and a heavy quark respectively.

I describe an effective point-particle action derived from the Polyakov action for a straight string in a semi-classical approximation. After quantisation the equation of motion gives rise to the spectrum of mesons consisting of a massless and a heavy quark. I evaluated the spectrum in the standard $\mathrm{AdS}_{5} \times \mathrm{S}^{5}$ background, where I could find an analytic formula for the numerically determined heavylight meson masses, and for the non-supersymmetric backgrounds by Constable-Myers [42] and by Gubser discussed earlier. In the for- B meson mer case a comparison with the experimental values of the B meson mass yields a deviation of about $20 \%$.

The models considered in this thesis are not meant to be realistic duals of QCD, but instead focus on a particular aspect like chiral symmetry breaking by a chiral quark condensate, the meson spectrum for D3/D7 AdS/CFT either non-supersymmetric deformed or with a Higgs VEV switched on, and the spectrum of heavy-light mesons in several backgrounds, giving a description of B mesons.

It would be certainly interesting to extend the techniques developed in future challenges this thesis to a more realistic example of AdS/QCD.* Over the last years there has been steady progress towards such a description, including string

*In particular the heavy-light meson construction could be easily extended to other, more realistic models. 
theory duals of theories that exhibit chiral symmetry breaking [82, 106124]. There are however three major points that need to be addressed in future refinements of AdS/QCD.

strong coupling The models considered here have a UV fixed point, but they are not asymptotically free. The weak-strong nature of the duality, which makes AdS/CFT so interesting, unfortunately means that weak coupling in the field theory's UV implies strong curvature towards the boundary of the AdS space, thus requiring a full string theoretical treatment, which currently is not feasible. Lacking that, there are recent attempts to circumvent the situation by introducing a UV cut-off in the geometry to produce phenomenological models of QCD dynamics [95-97, 125-139].

backreaction

A second problematic property is the probe limit $N_{f} \ll N_{c}$, which corresponds to the quenched approximation of lattice QFT. Additional contributions are roughly of the order $\frac{N_{f}}{N_{c}}$. Including the backreaction of the D7-branes on the geometry would allow the number of flavours to be of the same order of magnitude as the number of colours. Such backgrounds have been considered in [81].

separation of scales

The last important aspect is the separation of the SUSY and confinement scales. In the B physics example discussed in Section 5.3, the $\mathrm{B}$ meson is far in the supersymmetric regime. To change this situation one needs a background configuration that incorporates at least two different scales.

From the recent works cited above one can read off a tendency to focus on particular aspects of the larger problem of finding a holographic dual of QCD and YM theories, an approach also to be found in this thesis. A challenge for the future will be to incorporate into one model as many as possible of the insights gained here and elsewhere since the discovery of AdS/CFT duality almost ten years ago.

In the second Part of this thesis the coupling of supersymmetric quantum field theories to minimal supergravity was investigated. Coupling a gravity background to a conformal quantum field theory gives rise to a conformal anomaly 


$$
\left\langle T_{m}{ }^{m}\right\rangle=c C^{2}-a \tilde{\mathcal{R}}^{2}+b \mathcal{R}^{2}+f \square R
$$

In [48] a space-time dependent coupling approach was used to calculate consistency conditions for the coefficients in the two-dimensional anomaly providing an alternative proof for Zamolodchikov's $c$-theorem. However [48] did not obtain consistency conditions sufficiently restrictive to extend the theorem to four dimensions.

The specific project pursued here was to extend this technique to superfields and determine the conformal anomaly for those supersymmetric field theories whose coupling constants can be promoted to chiral fields $\lambda$. A prominent example for such is given by super-Yang-Mills theories.

The steps performed in detail were:

- I determined a complete ansatz for the conformal anomaly by finding basis of superfield a basis of 38 local superfield expressions of dimension 2 and compos- operators ing a linear combination with arbitrary coefficient functions $b(\lambda, \bar{\lambda})$. In the constant coupling limit, these coefficient functions become the superspace analogue of the coefficients $c, a, b$ and $f$ that appear in the bosonic conformal anomaly $(\star)$.

- Then I calculated the Wess-Zumino consistency conditions for the consistency coefficient functions, which arise from the fact that Weyl transfor- conditions mations are Abelian.

- Furthermore I discussed the dependence on local counterterms and possible consequences of S-duality in the $\mathcal{N}=4$ case.

- It is noted that a superfield version of the Riegert operator* is needed to make contact with an existing one-loop calculation [49]. Various superfield Riegert approaches to the problem of finding a superfield Riegert operator (which is independent of the anomaly calculation presented) have been discussed. The conclusion is that the problem is rooted in the $\mathrm{U}(1)_{R}$ symmetry being built into the formalism of minimal supergravity in superfield formulation in a local way, while on the component level the $\mathrm{U}(1)_{R}$ is only realised as a global symmetry.

In order to check this assumption it would be desirable to repeat the computer

*The Riegert operator is the unique conformally covariant differential operator of algebra? fourth order acting on a scalar field of Weyl weight 0 . 
full calculation in a component approach. The sheer size of this task is daunting however: The basis for the anomaly I found contains about 40 terms in superfield formulation plus their complex conjugates. As a consequence the calculation of the Wess-Zumino consistency conditions is very involved and potentially error prone. A component based approach will probably incorporate even more terms and should therefore be implemented with the help of a computer. Unfortunately a computer based treatment of supergravity has a number of requirements not satisfied by any existing computer algebra system (CAS) today. These requirements are

- an efficient mechanism for the representation of tensors and contracted indices,

- handling of commuting, anticommuting and non-commuting objects (this should include the ability to reduce a number of terms to a canonical basis of terms using the supergravity algebra and Bianchi identities),

- a way to represent non-commuting tensor valued functions of other objects (e.g. for non-anticommuting spinorial derivatives),

- making no assumption about the symmetries of the metric,

- allowing torsion, and

- no automatic expansion of compact parenthesised expressions into a lengthy sum of terms.

Of the existing systems, FORM [140] seems to be coming the closest to these requirements since it provides a rather low-level tensor support without restrictive internal assumptions. Its summarising capabilities are unsatisfactory however and may be a major obstacle in the implementation of a computer based analysis of the trace anomaly.

Another promising program is Cadabra [141, 142], which meets all of the above requirements but is still in a development stage.

component

Nevertheless the next steps in a future analysis of the trace anomaly calculation are the implementation of a supergravity computer algebra package and a component based analysis. As outlined above this is a difficult task, but 
the results presented in this thesis can serve as a highly non-trivial unit test to confirm the correctness of such a package. Then one may carry out a complete component expansion of all basis terms and reexamine the question of whether a superfield version of the Riegert operator does exist in minimal superfield supergravity. This analysis can then be easily extended to non-minimal SUGRA and as a check one may reproduce the Riegert operator in new-minimal SUGRA as well.

A reimplementation of the whole calculation in a component based approach would provide an independent source of confirmation for the results of this thesis. If a superfield based treatment of minimal supergravity is consistent on the quantum level, ${ }^{*}$ the two calculations should actually yield the same result, strengthening confidence in the results presented here. Of course inconsistency would be an interesting result in its own right.

In any case I hope to have provided a basis for understanding the structure of the conformal anomaly in supersymmetric field theories coupled to supergravity.

${ }^{*}$ See [53] on why a superfield treatment of minimal supergravity should be consistent and [54] on the question of consistence of anomaly calculations in the presence of compensating fields. 

It pays to be obvious, especially if you have a repu-

tation for subtlety.

Isaac Asimov

\section{Acknowledgements}

First of all I would like to thank Olga, who has carried the burden of raising our baby with almost no assistance on my part. I doubt having been a good father in Calista's first year, I know for sure that I should and can be a better husband. Second I would like to thank Calista for making me appreciate the simple things in life - like a few hours of uninterrupted sleep.

Third, I am coming closer to tradition now, I would like to thank my supervisor Johanna Erdmenger for - that also goes with the traditionher extended patience with stupid questions and slow progress. I am particularly grateful for many hours of talking about "local couplings" and her bolstering my morale in face of difficult calculations whenever necessary.

I am grateful to Dieter Lüst for a desk, a salary or two and the most beautiful Heisenberg office.

I am thankful to Hugh Osborn, Peter Breitenlohner, Sergei Kuzenko and Gabriel Lopez Cardoso for helpful discussions on the subjects of supergravity, conformal covariance and space-time dependent couplings.

Last but not least, many thanks also to Robert Eisenreich and Sofa Crack. They both have spent a lot of time on making me comfortable in exchange for a pair of hard elbows.

Part of this thesis was supported by the DFG Graduiertenkolleg "The Standard Model of Particle Physics - structure, precision tests and extensions" and the Max-Planck-Institut für Physik, München. 



\section{Appendix A}

\section{Determinant Expansion}

While most complicated backgrounds require working in the linearised approximation (that is expansion to quadratic order of the DBI action, see sec. 1.4.3), for the vacuum solution there are occasions where a full expansion of the determinant in the DBI action is needed. Since in string/M theory there is at most an eleven-dimensional metric, this can be easily done using a computer. However symbolic algebra programs like MATHEMATICA ${ }^{\circledR}$ or Maple ${ }^{\circledR}$ are sometimes not capable of simplifying the result sufficiently well to obtain an expression suitable for calculations by hand. In that case the following theorem, which is probably well known in the mathematics literature (even though I could not find it), can be useful as long as the metric is sufficiently simple. Since the formulation of the theorem is a bit hard to decode, studying the corollaries first might be helpful, in particular the last two corollaries, which are relevant for the pullback of a D7-brane.

Theorem 2 (Full Determinant Expansion).

Let $A, B$ be $N \times N$ matrices and $\mathbb{1}$ the corresponding unity matrix, then it holds

$$
\operatorname{det}[\mathbb{1}+A B]=\operatorname{det}_{m, n}\left[\delta_{m n}+A_{k_{m} m} B_{m k_{n}}\right] \quad(\text { no sum on } \mathrm{m}) \text {, }
$$


where on the right hand side, Einstein's convention is used on the indices $k_{m}$ after having evaluated the determinant in indices $m$ and $n$.

The right hand side may be formulated alternatively in the following manner:

$$
\operatorname{det}[\mathbb{1}+A B]=\sum_{k_{1}, \ldots, k_{N}} \operatorname{det}\left[\frac{1}{N} \delta_{m n}+A_{k_{m} m} B_{m k_{n}}\right] \quad \text { (no sum on m) }
$$

Proof.

$$
\begin{aligned}
\text { LHS } & =\operatorname{det}[\mathbb{1}+A B] \\
& =\operatorname{det}\left[\sum_{k}\left(\frac{1}{N} \delta_{m n}+A_{m k} B_{k n}\right)\right] \\
& =\sum_{\pi \in S_{N}} \operatorname{sgn} \pi \prod_{m} \sum_{k_{m}}\left(\frac{1}{N} \delta_{m n}+A_{m k_{m}} B_{k_{m} n}\right) \\
& =\sum_{k_{1}, \ldots, k_{N}} \sum_{\pi \in S_{N}} \operatorname{sgn} \pi \prod_{m}\left(\frac{1}{N} \delta_{m \pi(m)}+A_{m k_{m}} B_{k_{m} \pi(m)}\right) \\
& =\sum_{k_{1}, \ldots, k_{N}} \sum_{\pi \in S_{N}} \operatorname{sgn} \pi\left[\prod_{m} A_{m k_{m}} B_{k_{m} \pi(m)}+\right. \\
& \left.=\prod_{i} \frac{\delta_{i \pi(i)}}{N} \prod_{m_{m \neq i}} A_{m k_{m}} B_{k_{m} \pi(m)}+\ldots\right] \\
\prod_{k_{1}, \ldots, k_{N}} A_{m} & {\left[\operatorname{det} B+\prod_{i} \frac{\delta_{i \pi(i)}}{N A_{m k_{m}}} \operatorname{det} \mathcal{B}_{i i}+\ldots\right] }
\end{aligned}
$$

where $\mathcal{B}_{i i}$ is the adjugate matrix corresponding to $B_{i i}$.

$$
\begin{aligned}
\mathrm{RHS} & =\sum_{k_{1}, \ldots, k_{N}} \operatorname{det}\left[\frac{1}{N} \delta_{m n}+A_{k_{m} m} B_{m k_{n}}\right] \\
= & \sum_{k_{1}, \ldots, k_{N}} \sum_{\pi \in S_{N}} \operatorname{sgn} \pi \prod_{m}\left(\frac{1}{N} \delta_{m \pi(m)}+A_{k_{m} m} B_{m k_{\pi(m)}}\right) \\
= & \sum_{k_{1}, \ldots, k_{N}} \sum_{\pi \in S_{N}} \operatorname{sgn} \pi \prod_{m}\left(\frac{1}{N} \delta_{m \pi(m)}+A_{m k_{m}} B_{k_{\pi(m)} m}\right) \\
= & \sum_{k_{1}, \ldots, k_{N}} \sum_{\pi \in S_{N}} \operatorname{sgn} \pi\left[\prod_{m} A_{m k_{m}} B_{k_{\pi(m)} m}+\right. \\
& \left.+\prod_{i} \frac{\delta_{i \pi(i)}}{N} \prod_{\substack{m \\
m \neq i}} A_{m k_{m}} B_{k_{\pi(m)} m}+\ldots\right]
\end{aligned}
$$




$$
\begin{aligned}
& =\sum_{k_{1}, \ldots, k_{N}} \prod_{m} A_{m k_{m}}\left[\operatorname{det} B+\prod_{i} \frac{\delta_{i \pi(i)}}{N A_{m k_{m}}} \operatorname{det} \mathcal{B}_{i i}+\ldots\right] \\
& =\mathrm{LHS}
\end{aligned}
$$

Corollary 1 (Vector formulation).

For the matrices

$$
A=\left(\vec{a}_{1}, \ldots, \vec{a}_{N}\right), \quad B=\left(\begin{array}{c}
\vec{b}_{1}^{T} \\
\vdots \\
\vec{b}_{N}^{T}
\end{array}\right),
$$

theorem (A.1) reads

$$
\operatorname{det}\left[\mathbb{1}+\sum_{\lambda} \vec{a}_{\lambda} \otimes \vec{b}_{\lambda}\right]=\operatorname{det}\left[\delta_{m n}+\left(\vec{a}_{m}\right)_{k_{m}}\left(\vec{b}_{m}\right)_{k_{n}}\right]
$$

where again Einstein's convention is used on $k_{m}$.

Corollary 2 (Fewer vectors).

For $\vec{a}_{\lambda}=\vec{b}_{\lambda}=0 \quad \forall \lambda>L$ this is

$$
\begin{aligned}
\operatorname{det} & {\left[\mathbb{1}+\sum_{\lambda=1}^{L} \vec{a}_{\lambda} \otimes \vec{b}_{\lambda}\right] } \\
& =\operatorname{det}\left(\begin{array}{cc}
\delta_{m n}+\left(\vec{a}_{m}\right)_{k_{m}}\left(\vec{b}_{m}\right)_{k_{n}} & \text { irrelevant } \\
0 & \mathbb{1}_{N-L}
\end{array}\right) \\
& =\operatorname{det}\left[\delta_{\mu \nu}+\left(\vec{a}_{\mu}\right)_{k_{\mu}}\left(\vec{b}_{\mu}\right)_{k_{\nu}}\right],
\end{aligned}
$$

with indices $\mu, \nu$ running from 1 to $L$ and summation on indices $k_{\mu}$. The right hand side may be evaluated using the following simple MATHEMATICA script.

$1 \quad$ rank $=4 ; \quad(*$ plug in appropriate value $*)$

2 Expand $[\operatorname{Det}[\mathrm{Table}[\operatorname{dummy}[\mathbf{i}][j],\{i, \operatorname{rank}\},\{j, \operatorname{rank}\}]]] /$.

$$
\text { dummy }\left[\mathrm{x}_{-}\right]\left[\mathrm{y}_{-}\right]: \rightarrow \text { KroneckerDelta }[\mathrm{x}, \mathrm{y}]+\overrightarrow{\mathrm{a}}_{\mathrm{x}}[\mathrm{x}] \overrightarrow{\mathrm{b}}_{\mathrm{y}}[\mathrm{y}] \text {; }
$$

3 result $\left.=\left(\% / / \cdot \vec{a}_{i_{-}}\left[k_{-}\right] \vec{b}_{j_{-}}\left[k_{-}\right] \rightarrow \vec{a}_{i} \cdot \vec{b}_{j}\right]\right)$

Corollary 3 (D7 pullback for diagonal metric). 
For $L=2$ one obtains

$$
\begin{aligned}
\operatorname{det} & {\left[\mathbb{1}+\vec{a}_{1} \otimes \vec{b}_{1}+\vec{a}_{2} \otimes \vec{b}_{2}\right] } \\
& =\operatorname{det}\left(\begin{array}{cc}
1+\left(\vec{a}_{1}\right)_{k_{1}}\left(\vec{b}_{1}\right)_{k_{1}} & \left(\vec{a}_{1}\right)_{k_{1}}\left(\vec{b}_{1}\right)_{k_{2}} \\
\left(\vec{a}_{2}\right)_{k_{2}}\left(\vec{b}_{2}\right)_{k_{1}} & 1+\left(\vec{a}_{2}\right)_{k_{2}}\left(\vec{b}_{2}\right)_{k_{2}}
\end{array}\right) \\
& =\left(1+\vec{a}_{1} \vec{b}_{1}\right)\left(1+\vec{a}_{2} \vec{b}_{2}\right)-\left(\vec{a}_{2} \vec{b}_{1}\right)\left(\vec{a}_{1} \vec{b}_{2}\right),
\end{aligned}
$$

which for the vectors

$$
\begin{array}{ll}
\left(\vec{a}_{1}\right)_{b}=G_{88} G^{b c} \partial_{c} z^{8}, & \left(\vec{a}_{2}\right)_{b}=G_{99} G^{b c} \partial_{c} z^{9}, \\
\left(\vec{b}_{1}\right)_{a}=\partial_{a} z^{8}, & \left(\vec{b}_{2}\right)_{a}=\partial_{a} z^{9}
\end{array}
$$

yields

$$
\begin{aligned}
\operatorname{det}[ & \left.\mathbb{1}+G_{88} G^{b c} \partial_{c} z^{8} \partial_{a} z^{8}+G_{99} G^{b c} \partial_{c} z^{9} \partial_{a} z^{9}\right] \\
= & \left(1+G_{88} G^{b c} \partial_{b} z^{8} \partial_{c} z^{8}\right)\left(1+G_{99} G^{b c} \partial_{b} z^{9} \partial_{c} z^{9}\right) \\
& -G_{88} G_{99}\left(G^{b c} \partial_{b} z^{8} \partial_{c} z^{9}\right)^{2} .
\end{aligned}
$$

Corollary 4 (D7 pullback for block diagonal metric).

For a ten-dimensional metric of the form

$$
G_{A B} d X^{A} d X^{B}=\left(d x^{a} d z^{i}\right)\left(\begin{array}{cc}
g_{a b} & 0 \\
0 & g_{i j}
\end{array}\right)\left(\begin{array}{l}
d x^{b} \\
d z^{j}
\end{array}\right)
$$

with indices $a, b=0, \ldots, 7$ and $i, j=8,9$, the determinant of an eight dimensional pullback with respect to the embedding $x^{a}=\xi^{a}, z^{i}=z^{i}\left(\xi^{a}\right)$ is given by

$$
\begin{aligned}
\operatorname{det} & P\left[G_{A B}\right] \\
& =\operatorname{det}\left\{g_{a b}+g_{i j} \frac{\partial z^{i}}{\partial \xi^{a}} \frac{\partial z^{j}}{\partial \xi^{b}}\right\} \\
& =\operatorname{det} g_{a b} \cdot \operatorname{det}\left\{\mathbb{1}_{8}+g_{i j} \partial_{a} z^{i} \partial_{b} z^{j} g^{b c}\right\} \\
& =\operatorname{det} g_{a b} \cdot \operatorname{det}\left[\mathbb{1}+\sum_{\lambda=1}^{4} \vec{a}_{\lambda} \otimes \vec{b}_{\lambda}\right]
\end{aligned}
$$


with the vectors $\vec{a}_{\lambda}, \vec{b}_{\lambda}$ given by

$$
\begin{array}{ll}
\vec{a}_{1}:=g_{88}(g) \cdot \vec{\nabla} z^{8}, & \vec{b}_{1}:=\vec{\nabla} z^{8}, \\
\vec{a}_{2}:=g_{89}(g) \cdot \vec{\nabla} z^{8}, & \vec{b}_{2}:=\vec{\nabla} z^{9}, \\
\vec{a}_{3}:=g_{98}(g) \cdot \vec{\nabla} z^{9}=g_{98} / g_{88} \vec{a}_{1}, & \vec{b}_{3}:=\vec{\nabla} z^{8}=\vec{b}_{1}, \\
\vec{a}_{4}:=g_{99}(g) \cdot \vec{\nabla} z^{9}=g_{99} / g_{89} \vec{a}_{2}, & \vec{b}_{4}:=\vec{\nabla} z^{9}=\vec{b}_{2},
\end{array}
$$

where $(g) \cdot \vec{\nabla}$ denotes the matrix multiplication $g^{a b} \partial_{b}$. Using the theorem this may be expanded into $4 !=24$ terms, which due to above proportionality properties can be dramatically simplified, and one obtains

$$
\begin{aligned}
\operatorname{det} P\left[G_{A B}\right]=\operatorname{det} g_{c d} \cdot(1 & +g_{i j} \partial_{a} z^{i} \partial_{b} z^{j} g^{a b} \\
& \left.+\operatorname{det} g_{i j} \cdot \operatorname{det}_{k l}\left\{\partial_{a} z^{k} \partial_{b} z^{l} g^{a b}\right\}\right),
\end{aligned}
$$

where

$$
\begin{aligned}
\operatorname{det}_{k l} & \left\{\partial_{a} z^{k} \partial_{b} z^{l} g^{a b}\right\} \\
& =\left(g^{a b} \partial_{a} z^{8} \partial_{b} z^{8}\right)\left(g^{c d} \partial_{c} z^{8} \partial_{d} z^{8}\right)-\left(g^{a b} \partial_{a} z^{8} \partial_{b} z^{9}\right)^{2} .
\end{aligned}
$$

Note that no approximation has been used. 

Genius is one per cent inspiration, ninety-nine per cent perspiration.

\section{Appendix B}

\section{Weyl Variation of the Basis}

This Chapter provides the Weyl variations of all basis terms. The terms for $\Delta_{\sigma}^{W}$ can be extracted from those proportional to $\sigma$, and correspondingly for the complex conjugated fields.

$$
\begin{aligned}
E \delta\left[E^{-1} G_{\alpha \dot{\alpha}} G^{\alpha \dot{\alpha}}\right]= & 2 i G_{\alpha \dot{\alpha}} \mathcal{D}^{\alpha \dot{\alpha}}(\bar{\sigma}-\sigma) \\
E \delta\left[E^{-1} R \bar{R}\right]= & -\frac{1}{4}\left(\overline{\mathcal{D}}^{2} \bar{\sigma}\right) \bar{R}-\frac{1}{4}\left(\mathcal{D}^{2} \sigma\right) R \\
E \delta\left[E^{-1} R^{2}\right]= & 3(\bar{\sigma}-\sigma) R^{2}-\frac{1}{2}\left(\overline{\mathcal{D}}^{2} \bar{\sigma}\right) R \\
E \delta\left[E^{-1} \bar{R}^{2}\right]= & 3(\sigma-\bar{\sigma}) \bar{R}^{2}-\frac{1}{2}\left(\mathcal{D}^{2} \sigma\right) \bar{R} \\
E \delta\left[E^{-1} \mathcal{D}^{2} R\right]= & -2\left(\mathcal{D}^{\alpha} \sigma\right)\left(\mathcal{D}_{\alpha} R\right)-2\left(\mathcal{D}^{2} \sigma\right) R-\frac{1}{4} \mathcal{D}^{2} \overline{\mathcal{D}}^{2} \bar{\sigma} \\
= & -2\left(\mathcal{D}^{\alpha} \sigma\right)\left(\mathcal{D}_{\alpha} R\right)-2\left(\mathcal{D}^{2} \sigma\right) R \\
& +2\left(\mathcal{D}_{\alpha \dot{\alpha}} \mathcal{D}^{\alpha \dot{\alpha}} \bar{\sigma}\right)-2 i G_{\alpha \dot{\alpha}}\left(\mathcal{D}^{\alpha \dot{\alpha}} \bar{\sigma}\right) \\
E \delta\left[E^{-1} \overline{\mathcal{D}}^{2} \bar{R}\right]= & -2\left(\mathcal{D}_{\dot{\alpha}} \bar{\sigma}\right)\left(\overline{\mathcal{D}}^{\dot{\alpha}} \bar{R}\right)-2\left(\overline{\mathcal{D}}^{2} \bar{\sigma}\right) \bar{R}-\frac{1}{4} \overline{\mathcal{D}}^{2} \mathcal{D}^{2} \sigma \\
= & -2\left(\overline{\mathcal{D}}_{\dot{\alpha}} \bar{\sigma}\right)\left(\overline{\mathcal{D}}^{\dot{\alpha}} \bar{R}\right)-2\left(\overline{\mathcal{D}}^{2} \bar{\sigma}\right) \bar{R} \\
& +2\left(\mathcal{D}_{\alpha \dot{\alpha}} \mathcal{D}^{\alpha \dot{\alpha}} \sigma\right)+2 i G_{\alpha \dot{\alpha}}\left(\mathcal{D}^{\alpha \dot{\alpha}} \sigma\right) \\
E \delta\left[E^{-1} R \mathcal{D}^{2} \lambda\right]= & -\frac{1}{4}\left(\overline{\mathcal{D}}^{2} \bar{\sigma}\right)\left(\mathcal{D}^{2} \lambda\right)+2 R\left(\mathcal{D}^{\alpha} \sigma\right)\left(\mathcal{D}_{\alpha} \lambda\right) \\
E \delta\left[E^{-1} \bar{R}^{2} \bar{\lambda}\right]= & -\frac{1}{4}\left(\mathcal{D}^{2} \sigma\right)\left(\overline{\mathcal{D}}^{2} \bar{\lambda}\right)+2 \bar{R}\left(\overline{\mathcal{D}}_{\dot{\alpha}} \bar{\sigma}\right)\left(\overline{\mathcal{D}}^{\dot{\alpha}} \bar{\lambda}\right)
\end{aligned}
$$




$$
\begin{aligned}
& E \delta\left[E^{-1} R \overline{\mathcal{D}}^{2} \bar{\lambda}\right]=3(\bar{\sigma}-\sigma) R\left(\overline{\mathcal{D}}^{2} \bar{\lambda}\right)-\frac{1}{4}\left(\overline{\mathcal{D}}^{2} \bar{\sigma}\right)\left(\overline{\mathcal{D}}^{2} \bar{\lambda}\right) \\
& +2 R\left(\overline{\mathcal{D}}_{\dot{\alpha}} \bar{\sigma}\right)\left(\overline{\mathcal{D}}^{\dot{\alpha}} \bar{\lambda}\right) \\
& E \delta\left[E^{-1} \bar{R} \mathcal{D}^{2} \lambda\right]=3(\sigma-\bar{\sigma}) \bar{R}\left(\mathcal{D}^{2} \lambda\right)-\frac{1}{4}\left(\mathcal{D}^{2} \sigma\right)\left(\mathcal{D}^{2} \lambda\right) \\
& +2 \bar{R}\left(\mathcal{D}^{\alpha} \sigma\right)\left(\mathcal{D}_{\alpha} \lambda\right) \\
& E \delta\left[E^{-1}\left(\mathcal{D}^{\alpha} R\right)\left(\mathcal{D}_{\alpha} \lambda\right)\right]=-2\left(\mathcal{D}^{\alpha} \sigma\right)\left(\mathcal{D}_{\alpha} \lambda\right) R-\frac{1}{4}\left(\mathcal{D}^{\alpha} \overline{\mathcal{D}}^{2} \bar{\sigma}\right)\left(\mathcal{D}_{\alpha} \lambda\right) \\
& =-2\left(\mathcal{D}^{\alpha} \sigma\right)\left(\mathcal{D}_{\alpha} \lambda\right) R+\left[\left(G^{\alpha \dot{\alpha}}-i \mathcal{D}^{\alpha \dot{\alpha}}\right)\left(\overline{\mathcal{D}}_{\dot{\alpha}} \bar{\sigma}\right)\right]\left(\mathcal{D}_{\alpha} \lambda\right) \\
& E \delta\left[E^{-1}\left(\overline{\mathcal{D}}_{\dot{\alpha}} \bar{R}\right)\left(\overline{\mathcal{D}}^{\dot{\alpha}} \bar{\lambda}\right)\right]=-2\left(\overline{\mathcal{D}}_{\dot{\alpha}} \bar{\sigma}\right)\left(\overline{\mathcal{D}}^{\dot{\alpha}} \bar{\lambda}\right) \bar{R}-\frac{1}{4}\left(\overline{\mathcal{D}}_{\dot{\alpha}} \mathcal{D}^{2} \sigma\right)\left(\mathcal{D}^{\dot{\alpha}} \bar{\lambda}\right) \\
& =-2\left(\overline{\mathcal{D}}_{\dot{\alpha}} \bar{\sigma}\right)\left(\overline{\mathcal{D}}^{\dot{\alpha}} \bar{\lambda}\right) \bar{R}-\left[\left(G^{\alpha \dot{\alpha}}+i \mathcal{D}^{\alpha \dot{\alpha}}\right)\left(\mathcal{D}_{\alpha} \sigma\right)\right]\left(\overline{\mathcal{D}}_{\dot{\alpha}} \bar{\lambda}\right) \\
& E \delta\left[E^{-1} G_{\alpha \dot{\alpha}} \mathcal{D}^{\alpha \dot{\alpha}} \lambda\right]=i\left[\mathcal{D}_{\alpha \dot{\alpha}}(\bar{\sigma}-\sigma)\right]\left(\mathcal{D}^{\alpha \dot{\alpha}} \lambda\right) \\
& -\frac{i}{2} G_{\alpha \dot{\alpha}}\left(\overline{\mathcal{D}}^{\dot{\alpha}} \bar{\sigma}\right)\left(\mathcal{D}^{\alpha} \lambda\right) \\
& E \delta\left[E^{-1} G_{\alpha \dot{\alpha}} \mathcal{D}^{\alpha \dot{\alpha}} \bar{\lambda}\right]=i\left[\mathcal{D}_{\alpha \dot{\alpha}}(\bar{\sigma}-\sigma)\right]\left(\mathcal{D}^{\alpha \dot{\alpha}} \bar{\lambda}\right) \\
& -\frac{i}{2} G_{\alpha \dot{\alpha}}\left(\mathcal{D}^{\alpha} \sigma\right)\left(\overline{\mathcal{D}}^{\dot{\alpha}} \bar{\lambda}\right) \\
& E \delta\left[E^{-1}\left(\mathcal{D}^{\alpha \dot{\alpha}} \mathcal{D}_{\alpha \dot{\alpha}} \lambda\right)\right]=\left(\mathcal{D}^{\alpha \dot{\alpha}}(\sigma+\bar{\sigma})\right) \mathcal{D}_{\alpha \dot{\alpha}} \lambda-R\left(\mathcal{D}^{\alpha} \sigma\right)\left(\mathcal{D}_{\alpha} \lambda\right) \\
& -\frac{i}{2}\left(\overline{\mathcal{D}}^{\dot{\alpha}} \bar{\sigma}\right)\left(\mathcal{D}_{\alpha \dot{\alpha}} \mathcal{D}^{\alpha} \lambda\right)+G^{\alpha \dot{\alpha}}\left(\overline{\mathcal{D}}_{\dot{\alpha}} \bar{\sigma}\right)\left(\mathcal{D}_{\alpha} \lambda\right) \\
& E \delta\left[E^{-1}\left(\mathcal{D}^{\alpha \dot{\alpha}} \mathcal{D}_{\alpha \dot{\alpha}} \bar{\lambda}\right)\right]=\left(\mathcal{D}^{\alpha \dot{\alpha}}(\sigma+\bar{\sigma})\right) \mathcal{D}_{\alpha \dot{\alpha}} \bar{\lambda}-\bar{R}\left(\overline{\mathcal{D}}_{\dot{\alpha}} \bar{\sigma}\right)\left(\overline{\mathcal{D}}^{\dot{\alpha}} \bar{\lambda}\right) \\
& -\frac{i}{2}\left(\mathcal{D}^{\alpha} \sigma\right)\left(\mathcal{D}_{\alpha \dot{\alpha}} \overline{\mathcal{D}}^{\dot{\alpha}} \bar{\lambda}\right)-G^{\alpha \dot{\alpha}}\left(\mathcal{D}_{\alpha} \sigma\right)\left(\overline{\mathcal{D}}_{\dot{\alpha}} \bar{\lambda}\right) \\
& E \delta\left[E^{-1} R\left(\overline{\mathcal{D}}_{\dot{\alpha}} \bar{\lambda}\right)\left(\overline{\mathcal{D}}^{\dot{\alpha}} \bar{\lambda}\right)\right]=3(\bar{\sigma}-\sigma) R\left(\overline{\mathcal{D}}_{\dot{\alpha}} \bar{\lambda}\right)\left(\overline{\mathcal{D}}^{\dot{\alpha}} \bar{\lambda}\right) \\
& -\frac{1}{4}\left(\overline{\mathcal{D}}^{2} \bar{\sigma}\right)\left(\overline{\mathcal{D}}_{\dot{\alpha}} \bar{\lambda}\right)\left(\overline{\mathcal{D}}^{\dot{\alpha}} \bar{\lambda}\right) \\
& E \delta\left[E^{-1} G^{\alpha \dot{\alpha}}\left(\mathcal{D}_{\alpha} \lambda\right)\left(\overline{\mathcal{D}}_{\dot{\alpha}} \bar{\lambda}\right)\right]=i\left(\mathcal{D}_{\alpha \dot{\alpha}}(\bar{\sigma}-\sigma)\right)\left(\mathcal{D}^{\alpha} \lambda\right)\left(\overline{\mathcal{D}}^{\dot{\alpha}} \bar{\lambda}\right) \\
& E \delta\left[E^{-1}\left(\mathcal{D}_{\alpha \dot{\alpha}} \lambda\right)\left(\mathcal{D}^{\alpha \dot{\alpha}} \bar{\lambda}\right)\right]=-\frac{i}{2}\left(\overline{\mathcal{D}}_{\dot{\alpha}} \bar{\sigma}\right)\left(\mathcal{D}_{\alpha} \lambda\right)\left(\mathcal{D}^{\alpha \dot{\alpha}} \bar{\lambda}\right) \\
& -\frac{i}{2}\left(\mathcal{D}^{\alpha} \sigma\right)\left(\overline{\mathcal{D}}^{\dot{\alpha}} \bar{\lambda}\right)\left(\mathcal{D}_{\alpha \dot{\alpha}} \lambda\right) \\
& E \delta\left[E^{-1}\left(\mathcal{D}^{\alpha \dot{\alpha}} \lambda\right)\left(\mathcal{D}_{\alpha \dot{\alpha}} \lambda\right)\right]=-i\left(\overline{\mathcal{D}}^{\dot{\alpha}} \bar{\sigma}\right)\left(\mathcal{D}^{\alpha} \lambda\right)\left(\mathcal{D}_{\alpha \dot{\alpha}} \lambda\right) \\
& E \delta\left[E^{-1}\left(\mathcal{D}^{\alpha \dot{\alpha}} \bar{\lambda}\right)\left(\mathcal{D}_{\alpha \dot{\alpha}} \bar{\lambda}\right)\right]=-i\left(\mathcal{D}^{\alpha} \sigma\right)\left(\overline{\mathcal{D}}^{\dot{\alpha}} \bar{\lambda}\right)\left(\mathcal{D}_{\alpha \dot{\alpha}} \bar{\lambda}\right) \\
& E \delta\left[E^{-1}\left(\mathcal{D}^{\alpha} \lambda\right)\left(\mathcal{D}_{\alpha \dot{\alpha}} \overline{\mathcal{D}}^{\dot{\alpha}} \bar{\lambda}\right)\right]=\frac{1}{2}\left(\mathcal{D}^{\alpha} \lambda\right)\left[\left(\mathcal{D}_{\alpha \dot{\alpha}}(\bar{\sigma}+\sigma)\right)\left(\overline{\mathcal{D}}^{\dot{\alpha}} \bar{\lambda}\right)\right. \\
& \left.-i\left(\mathcal{D}_{\alpha} \sigma\right)\left(\overline{\mathcal{D}}^{2} \bar{\lambda}\right)+2\left(\overline{\mathcal{D}}^{\dot{\alpha}} \bar{\sigma}\right)\left(\mathcal{D}_{\alpha \dot{\alpha}} \bar{\lambda}\right)\right]
\end{aligned}
$$




$$
\begin{aligned}
& E \delta\left[E^{-1}(-1)\left(\overline{\mathcal{D}}^{\dot{\alpha}} \bar{\lambda}\right)\left(\mathcal{D}_{\alpha \dot{\alpha}} \mathcal{D}^{\alpha} \lambda\right)\right]=-\frac{1}{2}\left(\overline{\mathcal{D}}^{\dot{\alpha}} \bar{\lambda}\right)\left[\left(\mathcal{D}_{\alpha \dot{\alpha}}(\bar{\sigma}+\sigma)\right)\left(\mathcal{D}^{\alpha} \lambda\right)\right. \\
& \left.+i\left(\overline{\mathcal{D}}_{\dot{\alpha}} \bar{\sigma}\right)\left(\mathcal{D}^{2} \lambda\right)+2\left(\mathcal{D}^{\alpha} \sigma\right)\left(\mathcal{D}_{\alpha \dot{\alpha}} \lambda\right)\right] \\
& E \delta\left[E^{-1}\left(\mathcal{D}^{2} \lambda\right)\left(\overline{\mathcal{D}}^{2} \bar{\lambda}\right)\right]=2\left(\mathcal{D}^{\alpha} \sigma\right)\left(\mathcal{D}_{\alpha} \lambda\right)\left(\overline{\mathcal{D}}^{2} \bar{\lambda}\right) \\
& +2\left(\overline{\mathcal{D}}_{\dot{\alpha}} \bar{\sigma}\right)\left(\mathcal{D}^{2} \lambda\right)\left(\overline{\mathcal{D}}^{\dot{\alpha}} \bar{\lambda}\right) \\
& E \delta\left[E^{-1}\left(\mathcal{D}^{2} \lambda\right)^{2}\right]=3(\sigma-\bar{\sigma})\left(\mathcal{D}^{2} \lambda\right)^{2} \\
& +4\left(\mathcal{D}^{2} \lambda\right)\left(\mathcal{D}^{\alpha} \sigma\right)\left(\mathcal{D}_{\alpha} \lambda\right) \\
& E \delta\left[E^{-1}\left(\overline{\mathcal{D}}^{2} \bar{\lambda}\right)^{2}\right]=3(\bar{\sigma}-\sigma)\left(\overline{\mathcal{D}}^{2} \bar{\lambda}\right)^{2} \\
& +4\left(\overline{\mathcal{D}}^{2} \bar{\lambda}\right)\left(\overline{\mathcal{D}}_{\dot{\alpha}} \bar{\sigma}\right)\left(\overline{\mathcal{D}}^{\dot{\alpha}} \bar{\lambda}\right) \\
& E \delta\left[E^{-1}\left(\mathcal{D}^{\alpha} \lambda\right)\left(\mathcal{D}_{\alpha} \lambda\right)\left(\mathcal{D}^{2} \lambda\right)\right]=3(\sigma-\bar{\sigma})\left(\mathcal{D}^{\alpha} \lambda\right)\left(\mathcal{D}_{\alpha} \lambda\right)\left(\mathcal{D}^{2} \lambda\right) \\
& +2\left(\mathcal{D}^{\alpha} \sigma\right)\left(\mathcal{D}_{\alpha} \lambda\right)\left(\mathcal{D}^{\beta} \lambda\right)\left(\mathcal{D}_{\beta} \lambda\right) \\
& E \delta\left[E^{-1}\left(\overline{\mathcal{D}}_{\dot{\alpha}} \bar{\lambda}\right)\left(\overline{\mathcal{D}}^{\dot{\alpha}} \bar{\lambda}\right)\left(\overline{\mathcal{D}}^{2} \bar{\lambda}\right)\right]=3(\bar{\sigma}-\sigma)\left(\overline{\mathcal{D}}_{\dot{\alpha}} \bar{\lambda}\right)\left(\overline{\mathcal{D}}^{\dot{\alpha}} \bar{\lambda}\right)\left(\overline{\mathcal{D}}^{2} \bar{\lambda}\right) \\
& +2\left(\overline{\mathcal{D}}_{\dot{\alpha}} \bar{\sigma}\right)\left(\overline{\mathcal{D}}^{\dot{\alpha}} \bar{\lambda}\right)\left(\overline{\mathcal{D}}_{\dot{\beta}} \bar{\lambda}\right)\left(\overline{\mathcal{D}}^{\dot{\beta}} \bar{\lambda}\right) \\
& E \delta\left[E^{-1}\left(\mathcal{D}^{\alpha} \lambda\right)\left(\mathcal{D}_{\alpha} \lambda\right)\left(\overline{\mathcal{D}}^{2} \bar{\lambda}\right)\right]=2\left(\mathcal{D}^{\alpha} \lambda\right)\left(\mathcal{D}_{\alpha} \lambda\right)\left(\overline{\mathcal{D}}_{\dot{\alpha}} \bar{\sigma}\right)\left(\overline{\mathcal{D}}^{\dot{\alpha}} \bar{\lambda}\right) \\
& E \delta\left[E^{-1}\left(\overline{\mathcal{D}}_{\dot{\alpha}} \bar{\lambda}\right)\left(\mathcal{D}^{\dot{\alpha}} \bar{\lambda}\right)\left(\mathcal{D}^{2} \lambda\right)\right]=2\left(\overline{\mathcal{D}}_{\dot{\alpha}} \bar{\lambda}\right)\left(\overline{\mathcal{D}}^{\dot{\alpha}} \bar{\lambda}\right)\left(\mathcal{D}^{\alpha} \sigma\right)\left(\mathcal{D}_{\alpha} \lambda\right) \\
& E \delta\left[E^{-1}\left(\mathcal{D}_{\alpha \dot{\alpha}} \lambda^{i}\right)\left(\mathcal{D}^{\alpha} \lambda^{j}\right)\left(\overline{\mathcal{D}}^{\dot{\alpha}} \bar{\lambda}^{\bar{k}}\right)\right]=\frac{i}{2}\left(\mathcal{D}^{\alpha} \lambda^{j}\right)\left(\mathcal{D}_{\alpha} \lambda^{i}\right)\left(\overline{\mathcal{D}}_{\dot{\alpha}} \bar{\sigma}\right)\left(\overline{\mathcal{D}}^{\dot{\alpha}} \bar{\lambda}^{\bar{k}}\right) \\
& E \delta\left[E^{-1}\left(\mathcal{D}_{\alpha \dot{\alpha}} \bar{\lambda}^{\bar{\imath}}\right)\left(\mathcal{D}^{\alpha} \lambda^{k}\right)\left(\mathcal{D}^{\dot{\alpha}} \bar{\lambda}^{\bar{\jmath}}\right)\right]=-\frac{i}{2}\left(\overline{\mathcal{D}}_{\dot{\alpha}} \bar{\lambda}^{\bar{\imath}}\right)\left(\overline{\mathcal{D}}^{\dot{\alpha}} \bar{\lambda}^{\bar{\jmath}}\right)\left(\mathcal{D}^{\alpha} \sigma\right)\left(\mathcal{D}_{\alpha} \lambda^{k}\right) \\
& E \delta\left[E^{-1}\left(\mathcal{D}^{\alpha} \lambda\right)\left(\mathcal{D}_{\alpha} \lambda\right)\left(\overline{\mathcal{D}}_{\dot{\beta}} \bar{\lambda}\right)\left(\overline{\mathcal{D}}^{\dot{\beta}} \bar{\lambda}\right)\right]=0 \\
& E \delta\left[E^{-1}\left(\mathcal{D}^{\alpha} \lambda\right)\left(\mathcal{D}_{\alpha} \lambda\right)\left(\mathcal{D}^{\beta} \lambda\right)\left(\mathcal{D}_{\beta} \lambda\right)\right] \\
& =3(\sigma-\bar{\sigma})\left(\mathcal{D}^{\alpha} \lambda\right)\left(\mathcal{D}_{\alpha} \lambda\right)\left(\mathcal{D}^{\beta} \lambda\right)\left(\mathcal{D}_{\beta} \lambda\right) \\
& =3(\bar{\sigma}-\sigma)\left(\overline{\mathcal{D}}_{\dot{\alpha}} \bar{\lambda}\right)\left(\overline{\mathcal{D}}^{\dot{\alpha}} \bar{\lambda}\right)\left(\overline{\mathcal{D}}_{\dot{\beta}} \bar{\lambda}\right)\left(\overline{\mathcal{D}}^{\dot{\beta}} \bar{\lambda}\right)
\end{aligned}
$$



Vi Victa Vis.

Cicero

\section{Appendix C}

\section{Wess-Zumino}

\section{Consistency Condition}

\section{Weyl Contribution}

For the coefficients defined in

$$
\begin{aligned}
& \left(\Delta_{\sigma}^{W}\right)\left(\Delta_{\sigma^{\prime}}^{W}-\Delta_{\sigma^{\prime}}^{\beta}\right) W=\int d^{8} z E^{-1} \sigma^{\prime}\left\{\sigma \mathcal{C}_{0}+\left(\mathcal{D}^{\alpha} \sigma\right) \mathcal{C}_{\alpha}+\right. \\
& \left.\left(\mathcal{D}^{2} \sigma\right) \mathcal{C}_{2}+\left(\mathcal{D}^{\alpha \dot{\alpha}} \sigma\right) \mathcal{C}_{\alpha \dot{\alpha}}+\left(\mathcal{D}_{\alpha \dot{\alpha}} \mathcal{D}^{\alpha} \sigma\right) \mathcal{C}_{3}^{\dot{\alpha}}+\left(\mathcal{D}_{\alpha \dot{\alpha}} \mathcal{D}^{\alpha \dot{\alpha}} \sigma\right) \mathcal{C}_{4}\right\}
\end{aligned}
$$

one obtains

$$
\begin{aligned}
\mathcal{C}_{0}= & -3 b^{(C)} R^{2}+3 b^{(\bar{C})} \bar{R}^{2} \\
& -\left[3 R\left(\overline{\mathcal{D}}^{2} \bar{\lambda}\right) b^{(F)}\right]+3 \bar{R}\left(\mathcal{D}^{2} \lambda\right) b^{(\bar{F})} \\
& +3 b^{(K)} \bar{R}\left(\mathcal{D}^{\alpha} \lambda\right)\left(\mathcal{D}_{\alpha} \lambda\right)-\left[3 b^{(\bar{K})} R\left(\overline{\mathcal{D}}_{\dot{\alpha}} \bar{\lambda}\right)\left(\overline{\mathcal{D}}^{\dot{\alpha}} \bar{\lambda}\right)\right] \\
& +3 b^{(Q)}\left(\mathcal{D}^{2} \lambda\right)^{2}-3 b^{(\bar{Q})}\left(\overline{\mathcal{D}}^{2} \bar{\lambda}\right)^{2} \\
& +3\left(\mathcal{D}^{\alpha} \lambda\right)\left(\mathcal{D}_{\alpha} \lambda\right)\left(\mathcal{D}^{2} \lambda\right) b^{(R)}-3 b^{(\bar{R})}\left(\overline{\mathcal{D}}_{\dot{\alpha}} \bar{\lambda}\right)\left(\overline{\mathcal{D}}^{\dot{\alpha}} \bar{\lambda}\right)\left(\overline{\mathcal{D}}^{2} \bar{\lambda}\right) \\
& +3 b^{(V)}\left(\mathcal{D}^{\alpha} \lambda\right)\left(\mathcal{D}_{\alpha} \lambda\right)\left(\mathcal{D}^{\beta} \lambda\right)\left(\mathcal{D}_{\beta} \lambda\right)-3 b^{(\bar{V})}\left(\overline{\mathcal{D}}_{\dot{\alpha}} \bar{\lambda}\right)\left(\overline{\mathcal{D}}^{\dot{\alpha}} \bar{\lambda}\right)\left(\overline{\mathcal{D}}_{\dot{\beta}} \bar{\lambda}\right)\left(\overline{\mathcal{D}}^{\dot{\beta}} \bar{\lambda}\right),
\end{aligned}
$$




$$
\begin{aligned}
\mathcal{C}_{\alpha}= & -2 b^{(D)}\left(\mathcal{D}_{\alpha} R\right)-2 R\left(\mathcal{D}_{\alpha} \lambda\right) b^{(E)}+2 \bar{R}\left(\mathcal{D}_{\alpha} \lambda\right) b^{(\bar{F})} \\
& -\left[2\left(\mathcal{D}_{\alpha} \lambda\right) R b^{(G)}\right]-\left[G_{\alpha \dot{\alpha}}\left(\overline{\mathcal{D}}^{\dot{\alpha}} \bar{\lambda}\right) b^{(\bar{G})}\right] \\
& -\frac{i}{2} G_{\alpha \dot{\alpha}}\left(\overline{\mathcal{D}}^{\dot{\alpha}} \lambda\right) b^{(\bar{H})}-\frac{i}{2} b^{(M)}\left(\overline{\mathcal{D}}^{\dot{\alpha}} \bar{\lambda}\right)\left(\mathcal{D}_{\alpha \dot{\alpha}} \lambda\right)-i\left(\overline{\mathcal{D}}^{\dot{\alpha}} \bar{\lambda}\right)\left(\mathcal{D}_{\alpha \dot{\alpha}} \bar{\lambda}\right) b^{(\bar{N})} \\
& -\left[\frac{i}{2}\left(\mathcal{D}_{\alpha} \lambda\right)\left(\overline{\mathcal{D}}^{2} \bar{\lambda}\right) b^{(O)}\right]-\left[b^{(\bar{O})}\left(\mathcal{D}_{\alpha \dot{\alpha}} \lambda\right)\left(\overline{\mathcal{D}}^{\dot{\alpha}} \bar{\lambda}\right)\right]+2 b^{(P)}\left(\mathcal{D}_{\alpha} \lambda\right)\left(\overline{\mathcal{D}}^{2} \bar{\lambda}\right) \\
& +4 b^{(Q)}\left(\mathcal{D}^{2} \lambda\right)\left(\mathcal{D}_{\alpha} \lambda\right)+2\left(\mathcal{D}_{\alpha} \lambda\right)\left(\mathcal{D}^{\beta} \lambda\right)\left(\mathcal{D}_{\beta} \lambda\right) b^{(R)} \\
& +2 b^{(\bar{S})}\left(\overline{\mathcal{D}}_{\dot{\alpha}} \bar{\lambda}\right)\left(\overline{\mathcal{D}}^{\dot{\alpha}} \bar{\lambda}\right)\left(\mathcal{D}_{\alpha} \lambda\right)-\frac{i}{2} b^{(\bar{T})}\left(\overline{\mathcal{D}}_{\dot{\alpha}} \bar{\lambda}\right)\left(\overline{\mathcal{D}}^{\dot{\alpha}} \bar{\lambda}\right)\left(\mathcal{D}_{\alpha} \lambda\right) \\
\mathcal{C}_{2}= & -\frac{1}{4} b^{(B)} R-\frac{1}{2} b^{(\bar{C})} \bar{R} \\
& -2 b^{(D)} R-\frac{1}{4}\left(\overline{\mathcal{D}}^{2} \bar{\lambda}\right) b^{(\bar{E})} \\
& -\frac{1}{4}\left(\mathcal{D}^{2} \lambda\right) b^{(\bar{F})}-\frac{1}{4}\left(\overline{\mathcal{D}}_{\dot{\alpha}} \bar{\lambda}\right)\left(\overline{\mathcal{D}}^{\dot{\alpha}} \bar{\lambda}\right) b^{(\bar{J})}-\frac{1}{4} b^{(K)}\left(\mathcal{D}^{\alpha} \lambda\right)\left(\mathcal{D}_{\alpha} \lambda\right), \\
\mathcal{C}_{\alpha \dot{\alpha}}= & -2 i b^{(A)} G_{\alpha \dot{\alpha}}+2 i G_{\alpha \dot{\alpha}} b^{(\bar{D})} \\
& -i\left(\mathcal{D}_{\alpha \dot{\alpha}} \lambda\right) b^{(H)}-i\left(\mathcal{D}_{\alpha \dot{\alpha}} \bar{\lambda}\right) b^{(\bar{H})} \\
& -i b^{(L)}\left(\mathcal{D}_{\alpha} \lambda\right)\left(\overline{\mathcal{D}}_{\dot{\alpha}} \bar{\lambda}\right)+\left[\frac{1}{2} b^{(O)}\left(\mathcal{D}_{\alpha} \lambda\right)\left(\overline{\mathcal{D}}_{\dot{\alpha}} \bar{\lambda}\right)\right]-\left[\frac{1}{2} b^{(\bar{O})}\left(\mathcal{D}_{\alpha} \lambda\right)\left(\overline{\mathcal{D}}_{\dot{\alpha}} \bar{\lambda}\right)\right] \\
\overline{\mathcal{C}}_{3}^{\dot{\alpha}}= & {\left[i b^{(\bar{G})}\left(\overline{\mathcal{D}}^{\dot{\alpha}} \bar{\lambda}\right)\right] } \\
\mathcal{C}_{4}= & {\left.\left[2 b^{(\bar{D})}\right] .2 \mathrm{C} .2 \mathrm{~d}\right) }
\end{aligned}
$$

For further discussion, it proves useful to sort its contents with respect to derivatives on $\lambda$ or $\bar{\lambda}$.

$$
\begin{aligned}
\mathcal{C}_{0}= & -3 b^{(C)} R^{2}+3 b^{(\bar{C})} \bar{R}^{2} \\
& -\left[3 R\left(\overline{\mathcal{D}}^{2} \bar{\lambda}^{\bar{\imath}}\right) b_{\bar{\imath}}^{(F)}\right]+3 \bar{R}\left(\mathcal{D}^{2} \lambda^{i}\right) b_{i}^{(\bar{F})} \\
& +3 b_{i j}^{(K)} \bar{R}\left(\mathcal{D}^{\alpha} \lambda^{i}\right)\left(\mathcal{D}_{\alpha} \lambda^{j}\right)-\left[3 b_{\bar{\imath} \overline{\bar{\jmath}})}^{(\bar{K})} R\left(\overline{\mathcal{D}}_{\dot{\alpha}} \bar{\lambda}^{\bar{\imath}}\right)\left(\overline{\mathcal{D}}^{\dot{\alpha}} \bar{\lambda}^{\bar{\jmath}}\right)\right] \\
& +3 b_{i j}^{(Q)}\left(\mathcal{D}^{2} \lambda^{i}\right)\left(\mathcal{D}^{2} \lambda^{j}\right)-3 b_{\bar{\imath} \bar{\jmath}}^{(\bar{Q})}\left(\overline{\mathcal{D}}^{2} \bar{\lambda}^{\bar{\imath}}\right)\left(\overline{\mathcal{D}}^{2} \bar{\lambda}^{\bar{\jmath}}\right) \\
& +3\left(\mathcal{D}^{\alpha} \lambda^{i}\right)\left(\mathcal{D}_{\alpha} \lambda^{j}\right)\left(\mathcal{D}^{2} \lambda^{k}\right) b_{i j k}^{(R)}-3 b_{\bar{\imath} \bar{\jmath}}^{((\bar{R})}\left(\overline{\mathcal{D}}_{\dot{\alpha}} \bar{\lambda}^{\bar{\imath}}\right)\left(\overline{\mathcal{D}}^{\dot{\alpha}} \bar{\lambda}^{\bar{\jmath}}\right)\left(\overline{\mathcal{D}}^{2} \bar{\lambda}^{\bar{k}}\right) \\
& +3 b_{i j k l}^{(V)}\left(\mathcal{D}^{\alpha} \lambda^{i}\right)\left(\mathcal{D}_{\alpha} \lambda^{j}\right)\left(\mathcal{D}^{\beta} \lambda^{k}\right)\left(\mathcal{D}_{\beta} \lambda^{l}\right) \\
& -3 b_{\bar{\imath} \bar{\jmath} \bar{k} \bar{l}}^{(\overline{\mathcal{D}})}\left(\overline{\mathcal{D}}_{\dot{\alpha}} \bar{\lambda}^{\bar{\imath}}\right)\left(\overline{\mathcal{D}}^{\dot{\alpha}} \bar{\lambda}^{\bar{\jmath}}\right)\left(\overline{\mathcal{D}}_{\dot{\beta}} \bar{\lambda}^{\bar{k}}\right)\left(\overline{\mathcal{D}}^{\dot{\beta}} \bar{\lambda}^{\bar{l}}\right),
\end{aligned}
$$




$$
\begin{aligned}
& \mathcal{C}_{\alpha}=-2 b^{(D)}\left(\mathcal{D}_{\alpha} R\right)+\left(\mathcal{D}_{\alpha} \lambda^{i}\right)\left(-2 R\left(b_{i}^{(E)}+\left[b_{i}^{(G)}\right]+\left[\frac{1}{2} b_{i}^{(I)}\right]\right)+2 \bar{R} b_{i}^{(\bar{F})}\right) \\
& +\left(\overline{\mathcal{D}}^{\dot{\alpha}} \bar{\lambda}^{\bar{\imath}}\right)\left(-\frac{i}{2} G_{\alpha \dot{\alpha}} b_{\bar{\imath}}^{(\bar{H})}-G_{\alpha \dot{\alpha}} b_{\bar{\imath}}^{(\bar{I})}-\left[G_{\alpha \dot{\alpha}} b_{\bar{\imath}}^{(\bar{G})}\right]\right) \\
& +\left(\mathcal{D}_{\alpha \dot{\alpha}} \overline{\mathcal{D}}^{\dot{\alpha}} \bar{\lambda}^{\bar{\imath}}\right)\left(-\frac{i}{2} b_{\bar{\imath}}^{(\bar{I})}\right)+\left(\mathcal{D}_{\alpha \dot{\alpha}} \bar{\lambda}^{\bar{\imath}}\right)\left(\overline{\mathcal{D}}^{\dot{\alpha}} \bar{\lambda}^{\bar{\jmath}}\right)\left(-i b_{\bar{\imath}}^{(\bar{N})}\right) \\
& +\left(\mathcal{D}_{\alpha \dot{\alpha}} \lambda^{i}\right)\left(\overline{\mathcal{D}}^{\dot{\alpha}} \bar{\lambda}^{\bar{\jmath}}\right)\left(-\frac{i}{2} b_{i \bar{\jmath}}^{(M)}-\left[b_{\bar{\jmath} i}^{(\bar{O})}\right]\right) \\
& +\left(\mathcal{D}_{\alpha} \lambda^{i}\right)\left(\overline{\mathcal{D}}^{2} \bar{\lambda}^{\bar{\jmath}}\right)\left(-\left[\frac{i}{2} b_{i \bar{\jmath}}^{(O)}\right]+2 b_{i \bar{\jmath}}^{(P)}\right) \\
& +\left(\mathcal{D}^{2} \lambda^{i}\right)\left(\mathcal{D}_{\alpha} \lambda^{j}\right)\left(4 b_{i j}^{(Q)}\right) \\
& +\left(\mathcal{D}_{\alpha} \lambda^{i}\right)\left(\mathcal{D}^{\beta} \lambda^{j}\right)\left(\mathcal{D}_{\beta} \lambda^{k}\right)\left(2 b_{i j k}^{(R)}\right) \\
& +\left(\overline{\mathcal{D}}_{\dot{\alpha}} \bar{\lambda}^{\bar{\imath}}\right)\left(\overline{\mathcal{D}}^{\dot{\alpha}} \bar{\lambda}^{\bar{\jmath}}\right)\left(\mathcal{D}_{\alpha} \lambda^{k}\right)\left(2 b_{\bar{\imath} \bar{\jmath} k}^{(\bar{S})}-\frac{i}{2} b_{\{\bar{\imath} \bar{\jmath}\} k}^{(\bar{T})}\right), \\
& \mathcal{C}_{2}=-\left(\frac{1}{4} b^{(B)}+2 b^{(D)}\right) R-\frac{1}{2} b^{(\bar{C})} \bar{R}-\frac{1}{4}\left(\overline{\mathcal{D}}^{2} \bar{\lambda}^{\bar{\imath}}\right) b_{\bar{\imath}}^{(\bar{E})} \\
& -\frac{1}{4}\left(\mathcal{D}^{2} \lambda^{i}\right) b_{i}^{(\bar{F})}-\frac{1}{4}\left(\overline{\mathcal{D}}_{\dot{\alpha}} \bar{\lambda}^{\bar{\imath}}\right)\left(\overline{\mathcal{D}}^{\dot{\alpha}} \bar{\lambda}^{\bar{\jmath}}\right) b_{\bar{\imath} \bar{\jmath}}^{(\bar{J})}-\frac{1}{4} b_{i j}^{(K)}\left(\mathcal{D}^{\alpha} \lambda^{i}\right)\left(\mathcal{D}_{\alpha} \lambda^{j}\right), \\
& \mathcal{C}_{\alpha \dot{\alpha}}=2 i G_{\alpha \dot{\alpha}}\left(\left[b^{(\bar{D})}\right]-b^{(A)}\right)+\left(\mathcal{D}_{\alpha \dot{\alpha}} \lambda^{i}\right)\left(\left[b_{i}^{(I)}\right]-\left[i b_{i}^{(H)}\right]\right) \\
& +\left(\mathcal{D}_{\alpha \dot{\alpha}} \bar{\lambda}^{\bar{\imath}}\right)\left(b_{\bar{\imath}}^{(\bar{I})}-i b_{\bar{\imath}}^{(\bar{H})}\right)+\left(\left[\frac{1}{2} b_{i \bar{\jmath}}^{(O)}\right]-\left[\frac{1}{2} b_{i \bar{\jmath}}^{(\bar{O})}\right]-i b_{i \bar{\jmath}}^{(L)}\right)\left(\mathcal{D}^{\alpha} \lambda^{i}\right)\left(\overline{\mathcal{D}}^{\dot{\alpha}} \bar{\lambda}^{\bar{\jmath}}\right), \\
& \overline{\mathcal{C}}_{3}^{\dot{\alpha}}=\left[i b_{\bar{\jmath}}^{(\bar{G})}\left(\overline{\mathcal{D}}^{\dot{\alpha}} \bar{\lambda}^{\bar{\jmath}}\right)\right] \\
& \mathcal{C}_{4}=\left[2 b^{(\bar{D})}\right] \text {. }
\end{aligned}
$$

\section{Beta Contribution}

Acting with the operator $\Delta^{\beta}$ on the conformal anomaly, one first notices, that $\frac{\delta}{\delta \lambda^{i}}$ should only act on derivatives of $\lambda$, since otherwise a $\sigma \sigma^{\prime}$ contribution, which vanishes from the commutator, is created.

$$
\begin{aligned}
& \Delta_{\sigma}^{\beta}\left(\Delta_{\sigma^{\prime}}^{W}-\Delta_{\sigma^{\prime}}^{\beta}\right) W=\int d^{8} z E^{-1} \sigma^{\prime}\left\{\sigma \beta^{i}\left(\partial_{i} \mathcal{B}_{(0)}\right) \mathcal{A}_{(0)}+\left(\mathcal{D}^{\alpha} \sigma \beta^{i}\right) \mathcal{E}_{\alpha}^{i}\right. \\
& \left.+\left(\mathcal{D}^{2} \sigma \beta^{i}\right) \mathcal{E}_{(2)}^{i}+\left(\mathcal{D}^{\alpha \dot{\alpha}} \sigma \beta^{i}\right) \mathcal{E}_{\alpha \dot{\alpha}}^{i}+\left(\mathcal{D}_{\alpha \dot{\alpha}} \mathcal{D}^{\alpha \dot{\alpha}} \sigma \beta^{i}\right) \mathcal{E}_{4}^{i}+\left(\mathcal{D}_{\alpha \dot{\alpha}} \mathcal{D}^{\dot{\alpha}} \sigma \beta^{i}\right) \overline{\mathcal{E}}_{3}^{i \dot{\alpha}}\right\}
\end{aligned}
$$




$$
\begin{aligned}
\mathcal{E}_{\alpha}= & {\left[b_{i}^{(G)}\left(\mathcal{D}_{\alpha} R\right)\right]+\left[2 b_{i j}^{(J)} R\left(\mathcal{D}_{\alpha} \lambda^{j}\right)\right]+2 b_{i j}^{(K)} \bar{R}\left(\mathcal{D}_{\alpha} \lambda^{j}\right) } \\
& +b_{i \bar{\jmath}}^{(L)} G_{\alpha \dot{\alpha}}\left(\overline{\mathcal{D}}^{\dot{\alpha}} \bar{\lambda}^{\bar{\jmath}}\right)+\left[b_{i \bar{\jmath}}^{(O)}\left(\mathcal{D}_{\alpha \dot{\alpha}} \overline{\mathcal{D}}^{\dot{\alpha}} \bar{\lambda}^{\bar{\jmath}}\right)\right] \\
& +2 b_{i j k}^{(R)}\left(\mathcal{D}_{\alpha} \lambda^{j}\right)\left(\mathcal{D}^{2} \lambda^{k}\right)+\left[2 b_{i j \bar{k}}^{(S)}\left(\mathcal{D}_{\alpha} \lambda^{j}\right)\left(\overline{\mathcal{D}}^{2} \bar{\lambda}^{\bar{k}}\right)\right] \\
& +b_{j i \bar{k}}^{(T)}\left(\mathcal{D}_{\alpha \dot{\alpha}} \lambda^{j}\right)\left(\overline{\mathcal{D}}^{\dot{\alpha}} \bar{\lambda}^{\bar{k}}\right)+b_{\bar{\jmath} i \bar{k}}^{(\bar{T})}\left(\mathcal{D}^{\alpha \dot{\alpha}} \bar{\lambda}^{\bar{\jmath}}\right)\left(\overline{\mathcal{D}}_{\dot{\alpha}} \bar{\lambda}^{\bar{k}}\right) \\
& +2 b_{i j \bar{k} \bar{l}}^{(U)}\left(\mathcal{D}_{\alpha} \lambda^{j}\right)\left(\overline{\mathcal{D}}_{\dot{\beta}} \bar{\lambda}^{\bar{k}}\right)\left(\overline{\mathcal{D}}^{\dot{\beta}} \bar{\lambda}^{\bar{l}}\right)+4 b_{i j k l}^{(V)}\left(\mathcal{D}_{\alpha} \lambda^{j}\right)\left(\mathcal{D}^{\beta} \lambda^{k}\right)\left(\mathcal{D}_{\beta} \lambda^{l}\right) \\
\mathcal{E}_{2}= & b_{i}^{(E)} R+b_{i}^{(\bar{F})} \bar{R}+b_{i \bar{\jmath}}^{(P)}\left(\overline{\mathcal{D}}^{2} \bar{\lambda}^{\bar{\jmath}}\right) \\
& +2 b_{i j}^{(Q)}\left(\mathcal{D}^{2} \lambda^{j}\right)+b_{l j i}^{(R)}\left(\mathcal{D}^{\alpha} \lambda^{l}\right)\left(\mathcal{D}_{\alpha} \lambda^{j}\right)+b_{\bar{\imath} \bar{\jmath} i}^{(\bar{S})}\left(\overline{\mathcal{D}}_{\dot{\alpha}} \bar{\lambda}^{\bar{l}}\right)\left(\overline{\mathcal{D}}^{\dot{\alpha}} \bar{\lambda}^{\bar{\jmath}}\right) \\
\mathcal{E}_{\alpha \dot{\alpha}}= & {\left[b_{i}^{(H)} G_{\alpha \dot{\alpha}}\right]+b_{i \bar{\jmath}}^{(M)}\left(\mathcal{D}_{\alpha \dot{\alpha}} \bar{\lambda}^{\bar{\jmath}}\right)+b_{i j}^{(N)}\left(\mathcal{D}_{\alpha \dot{\alpha}} \lambda^{j}\right)+b_{i j \bar{k}}^{(T)}\left(\mathcal{D}_{\alpha} \lambda^{j}\right)\left(\overline{\mathcal{D}}_{\dot{\alpha}} \bar{\lambda}^{\bar{k}}\right), } \\
\overline{\mathcal{E}}_{3}^{\dot{\alpha}}= & {\left[b_{\bar{\jmath} i}^{(\bar{O})}\left(\overline{\mathcal{D}}^{\dot{\alpha}} \bar{\lambda}^{\bar{\jmath}}\right)\right] } \\
\mathcal{E}_{4}= & {\left[b_{i}^{(I)}\right] }
\end{aligned}
$$

\section{Summary}

The results of the previous two Sections can be used to determine the $\mathcal{F}$ coefficients defined by

$$
\begin{aligned}
\left(\Delta_{\sigma}^{W}-\Delta_{\sigma}^{\beta}\right)\left(\Delta_{\sigma^{\prime}}^{W}-\Delta_{\sigma^{\prime}}^{\beta}\right) W & \\
=\int d^{8} z E^{-1} \sigma^{\prime}\{ & \sigma \mathcal{F}_{0}+\left(\mathcal{D}^{\alpha} \sigma\right) \mathcal{F}_{\alpha}+\left(\mathcal{D}^{2} \sigma\right) \mathcal{F}_{2}+\left(\mathcal{D}^{\alpha \dot{\alpha}} \sigma\right) \mathcal{F}_{\alpha \dot{\alpha}} \\
& \left.+\left(\mathcal{D}_{\alpha \dot{\alpha}} \mathcal{D}^{\alpha} \sigma\right) \overline{\mathcal{F}}_{3}^{\dot{\alpha}}+\left(\mathcal{D}_{\alpha \dot{\alpha}} \mathcal{D}^{\alpha \dot{\alpha}} \sigma\right) \mathcal{F}_{4}\right\}
\end{aligned}
$$

by expanding the Weyl and beta contribution in terms of derivatives on $\lambda$ and $\bar{\lambda}$, keeping in mind that the $b$ coefficients and beta functions are functions of $\lambda$ and $\bar{\lambda}$ in general, so it holds

$$
\begin{aligned}
b & =b(\lambda, \bar{\lambda}) \\
\mathcal{D}_{\alpha} b & =\left(\mathcal{D}_{\alpha} \lambda^{k}\right)\left(\partial_{k} b\right), \\
\overline{\mathcal{D}}^{\dot{\alpha}} b & =\left(\overline{\mathcal{D}}^{\dot{\alpha}} \bar{\lambda}^{\bar{k}}\right)\left(\partial_{\bar{k}} b\right), \\
\mathcal{D}_{\alpha \dot{\alpha}} b & =\left(\mathcal{D}_{\alpha \dot{\alpha}} \lambda^{k}\right)\left(\partial_{k} b\right)+\left(\mathcal{D}_{\alpha \dot{\alpha}} \bar{\lambda}^{\bar{k}}\right)\left(\partial_{\bar{k}} b\right),
\end{aligned}
$$




$$
\begin{aligned}
\overline{\mathcal{D}}^{\dot{\alpha}} \mathcal{D}_{\alpha \dot{\alpha}} b= & \left(\overline{\mathcal{D}}^{\dot{\alpha}} \mathcal{D}_{\alpha \dot{\alpha}} \lambda^{k}\right)\left(\partial_{k} b\right)+\left(\mathcal{D}_{\alpha \dot{\alpha}} \lambda^{k}\right)\left(\overline{\mathcal{D}}^{\dot{\alpha}} \bar{\lambda}^{\bar{\jmath}}\right)\left(\partial_{\bar{\jmath}} \partial_{k} b\right) \\
& +\left(\overline{\mathcal{D}}^{\dot{\alpha}} \mathcal{D}_{\alpha \dot{\alpha}} \bar{\lambda}^{\bar{k}}\right)\left(\partial_{\bar{k}} b\right)+\left(\mathcal{D}_{\alpha \dot{\alpha}} \bar{\lambda}^{\bar{k}}\right)\left(\overline{\mathcal{D}}^{\dot{\alpha}} \bar{\lambda}^{\bar{\jmath}}\right)\left(\partial_{\bar{\jmath}} \partial_{\bar{k}} b\right), \\
\mathcal{D}^{\alpha \dot{\alpha}} \mathcal{D}_{\alpha \dot{\alpha}} b= & \left(\mathcal{D}^{\alpha \dot{\alpha}} \mathcal{D}_{\alpha \dot{\alpha}} \lambda^{k}\right)\left(\partial_{k} b\right)+\left(\mathcal{D}^{\alpha \dot{\alpha}} \mathcal{D}_{\alpha \dot{\alpha}} \bar{\lambda}^{\bar{k}}\right)\left(\partial_{\bar{k}} b\right) \\
& +\left(\mathcal{D}^{\alpha \dot{\alpha}} \lambda^{k}\right)\left(\mathcal{D}_{\alpha \dot{\alpha}} \lambda^{l}\right)\left(\partial_{l} \partial_{k} b\right) \\
& +2\left(\mathcal{D}^{\alpha \dot{\alpha}} \lambda^{k}\right)\left(\mathcal{D}_{\alpha \dot{\alpha}} \bar{\lambda}^{\bar{l}}\right)\left(\partial_{k} \partial_{\bar{l}} b\right) \\
& +\left(\mathcal{D}^{\alpha \dot{\alpha}} \bar{\lambda}^{\bar{k}}\right)\left(\mathcal{D}_{\alpha \dot{\alpha}} \bar{\lambda}^{\bar{l}}\right)\left(\partial_{\bar{k}} \partial_{\bar{l}} b\right)
\end{aligned}
$$

and similarly for $\beta^{i}$. This yields

$$
\begin{aligned}
\mathcal{F}_{0}= & \mathcal{C}_{0}+\beta^{i}\left(\partial_{i} \mathcal{B}\right) \cdot \mathcal{A} \\
+ & \left(\mathcal{D}^{\alpha} \lambda^{j}\right)\left(\partial_{j} \beta^{i}\right) \mathcal{E}_{\alpha}^{i} \\
+ & {\left[\left(\mathcal{D}^{2} \lambda^{j}\right)\left(\partial_{j} \beta^{i}\right)+\left(\mathcal{D}^{\alpha} \lambda^{j}\right)\left(\mathcal{D}_{\alpha} \lambda^{k}\right)\left(\partial_{j} \partial_{k} \beta^{i}\right)\right] \mathcal{E}_{2}^{i} } \\
+ & {\left[\left(\mathcal{D}^{\alpha \dot{\alpha}} \lambda^{j}\right)\left(\partial_{j} \beta^{i}\right)+\left(\mathcal{D}^{\alpha \dot{\alpha}} \bar{\lambda}^{\bar{\jmath}}\right)\left(\partial_{\bar{\jmath}} \beta^{i}\right)\right] \mathcal{E}_{\alpha \dot{\alpha}}^{i} } \\
+ & {\left[\left(\mathcal{D}_{\alpha \dot{\alpha}} \mathcal{D}^{\alpha} \lambda^{j}\right)\left(\partial_{j} \beta^{i}\right)+\left(\mathcal{D}^{\alpha} \lambda^{j}\right)\left(\mathcal{D}_{\alpha \dot{\alpha}} \lambda^{k}\right)\left(\partial_{k} \partial_{j} \beta^{i}\right)\right.} \\
& \left.+\left(\mathcal{D}^{\alpha} \lambda^{j}\right)\left(\mathcal{D}_{\alpha \dot{\alpha}} \bar{\lambda}^{\bar{k}}\right)\left(\partial_{\bar{k}} \partial_{j} \beta^{i}\right)\right] \overline{\mathcal{E}}_{3}^{\dot{\alpha} i} \\
+ & {\left[\left(\mathcal{D}^{\alpha \dot{\alpha}} \mathcal{D}_{\alpha \dot{\alpha}} \lambda^{k}\right)\left(\partial_{k} \beta^{i}\right)+\left(\mathcal{D}^{\alpha \dot{\alpha}} \mathcal{D}_{\alpha \dot{\alpha}} \bar{\lambda}^{\bar{k}}\right)\left(\partial_{\bar{k}} \beta^{i}\right)\right.} \\
& +\left(\mathcal{D}^{\alpha \dot{\alpha}} \lambda^{k}\right)\left(\mathcal{D}_{\alpha \dot{\alpha}} \lambda^{j}\right)\left(\partial_{j} \partial_{k} \beta^{i}\right)+2\left(\mathcal{D}^{\alpha \dot{\alpha}} \lambda^{k}\right)\left(\mathcal{D}_{\alpha \dot{\alpha}} \bar{\lambda}^{\bar{\jmath}}\right)\left(\partial_{\bar{\jmath}} \partial_{k} \beta^{i}\right) \\
& \left.+\left(\mathcal{D}^{\alpha \dot{\alpha}} \bar{\lambda}^{\bar{k}}\right)\left(\mathcal{D}_{\alpha \dot{\alpha}} \bar{\lambda}^{\bar{\jmath}}\right)\left(\partial_{\bar{\jmath}} \partial_{\bar{k}} \beta^{i}\right)\right] \mathcal{E}_{4}^{i}, \\
\mathcal{F}_{\alpha}= & \mathcal{C}_{\alpha}+\beta^{i} \mathcal{E}_{\alpha}^{i}+2\left(\mathcal{D}_{\alpha} \lambda^{j}\right)\left(\partial_{j} \beta^{i}\right) \mathcal{E}_{2}^{i} \\
+ & {\left[\left(\mathcal{D}_{\alpha \dot{\alpha}} \lambda^{k}\right)\left(\partial_{k} \beta^{i}\right)+\left(\mathcal{D}_{\alpha \dot{\alpha}} \bar{\lambda}^{\bar{k}}\right)\left(\partial_{\bar{k}} \beta^{i}\right)\right] \overline{\mathcal{E}}_{3}^{\dot{\alpha} i}, } \\
\mathcal{F}_{2}= & \mathcal{C}_{2}+\beta^{i} \mathcal{E}_{2}^{i}, \\
\mathcal{F}_{\alpha \dot{\alpha}}= & \mathcal{C}_{\alpha \dot{\alpha}}+\beta^{i} \mathcal{E}_{\alpha \dot{\alpha}}^{i}+\left(\mathcal{D}_{\alpha} \lambda^{j}\right)\left(\partial_{j} \beta^{i}\right) \overline{\mathcal{E}}_{3 \dot{\alpha}}^{i} \\
+ & 2\left(\mathcal{D}_{\alpha \dot{\alpha}} \lambda^{k}\right)\left(\partial_{k} \beta^{i}\right) \mathcal{E}_{4}^{i}+2\left(\mathcal{D}_{\alpha \dot{\alpha}} \bar{\lambda}^{\bar{k}}\right)\left(\partial_{\bar{k}} \beta^{i}\right) \mathcal{E}_{4}^{i}, \\
\overline{\mathcal{F}}_{3}^{\dot{\alpha}}= & \overline{\mathcal{C}}_{3}^{\dot{\alpha}}+\beta^{i} \overline{\mathcal{E}}_{3}^{i \dot{\alpha}}, \\
\mathcal{F}_{4}= & \mathcal{C}_{4}+\beta^{i} \mathcal{E}_{4}^{i} . \\
&
\end{aligned}
$$



I have made this longer, because I have not had the time to make it shorter.

Blaise Pascal, "Lettres provinciales"

\section{Appendix D}

\section{Coefficient Consistency \\ Equations}

Consistency equation (8.35e) yields

$$
2 \bar{b}^{(D)}-2 b^{(D)}+\beta^{i} \bar{b}_{i}^{(\bar{I})}-\bar{\beta}^{\bar{\imath}} \bar{b}_{\bar{\imath}}^{(\bar{I})}=0 .
$$

From consistency equation (8.35d) one obtains

$$
\begin{aligned}
& -\bar{b}^{(A)}+b^{(A)}=0 \\
& \bar{b}_{i j}^{(\bar{N})} \beta^{i}+\left(\partial_{j} \beta^{i}\right) \bar{b}_{i}^{(\bar{I})}+2 \partial_{j} \bar{b}^{(D)}+\left(\partial_{j} \bar{b}_{i}^{(\bar{I})}\right) \beta^{i}+b_{j}^{(I)}+i b_{j}^{(H)}+b_{j \bar{\imath}}^{(M)} \bar{\beta}^{\bar{\imath}} \\
& \quad+\left(\partial_{j} \bar{\beta}^{\bar{\imath}}\right) b_{\bar{\imath}}^{(\bar{I})}+2\left(\partial_{j} b^{(D)}\right)+\left(\partial_{j} b_{\bar{\imath}}^{(\bar{I})}\right) \bar{\beta}^{\bar{\imath}}-2 b_{j}^{(G)}-2 i \bar{\beta}^{\bar{\imath}} b_{j \bar{\imath}}^{(O)}=0 \\
& \quad b_{\bar{\imath} \bar{\jmath}}^{(\bar{N})} \beta^{\bar{\imath}}+\left(\partial_{\bar{\jmath}} \bar{\beta}^{\bar{\imath}}\right) b_{\bar{\imath}}^{(\bar{I})}+2 \partial_{\bar{\jmath}} b^{(D)}+\left(\partial_{\bar{\jmath}} b_{\bar{\imath}}^{(\bar{I})}\right) \bar{\beta}^{\bar{\imath}}+\bar{b}_{\bar{\jmath}}^{(I)}-i \bar{b}_{\bar{\jmath}}^{(H)}+\bar{b}_{i \bar{\jmath}}^{(M)} \beta^{i} \\
& \quad+\left(\partial_{\bar{\jmath}} \beta^{i}\right) \bar{b}_{i}^{(\bar{I})}+2\left(\partial_{\bar{\jmath}} \bar{b}^{(D)}\right)+\left(\partial_{\bar{\jmath}} \bar{b}_{i}^{(\bar{I})}\right) \beta^{i}-2 \bar{b}_{\bar{\jmath}}^{(G)}+2 i \beta^{i} \bar{b}_{\bar{\jmath} i}^{(O)}=0 \\
& -i \bar{b}_{i \bar{\jmath}}^{(L)}+\bar{b}_{k i \bar{\jmath}}^{(\bar{T})} \beta^{k}-i\left(\partial_{i} \bar{b}_{\bar{\jmath}}^{(G)}\right)-\left(\partial_{i} \bar{b}_{\bar{\jmath} k}^{(O)}\right) \beta^{k}+i b_{\bar{\jmath} i}^{(L)} \\
& \quad+b_{\bar{k} \bar{\jmath} i}^{(\bar{T})} \bar{\beta}^{\bar{k}}+i\left(\partial_{\bar{\jmath}} b_{i}^{(G)}\right)-\left(\partial_{\bar{\jmath}} b_{i \bar{k}}^{(O)}\right) \bar{\beta}^{\bar{k}}=0 .
\end{aligned}
$$


The following sets of equations have to be augmented by their complex conjugates. From consistency condition (8.35a) one gets

$$
\begin{aligned}
& -2 b^{(D)}+\frac{1}{4} b^{(B)}+b^{(A)}+2 b^{(D)}-\beta^{i} b_{i}^{(E)}=0, \\
& -2 b_{j}^{(E)}+2 \beta^{i} b_{i j}^{(K)}+b_{i}^{(E)}\left(\partial_{j} \beta^{i}\right)+\frac{1}{4}\left(\partial_{j} b^{(B)}\right) \\
& +2\left(\partial_{j} b^{(D)}\right)-\beta^{i}\left(\partial_{j} b_{i}^{(E)}\right)+b_{i j}^{(N)} \beta^{i}=0, \\
& 2 b_{j}^{(\bar{F})}+b_{i}^{(\bar{F})}\left(\partial_{j} \beta^{i}\right)+\frac{1}{2}\left(\partial_{j} b^{(\bar{C})}\right) \\
& +b_{j}^{(\bar{F})}-\beta^{i}\left(\partial_{j} b_{i}^{(\bar{F})}\right)+8 \beta^{i} b_{i j}^{(Q)}=0, \\
& -\frac{i}{2} b_{\bar{\jmath}}^{(\bar{H})}-b_{\bar{\jmath}}^{(\bar{I})}+\beta^{i} b_{i \bar{\jmath}}^{(L)}+b_{\bar{\jmath}}^{(\bar{E})}-4 \beta^{i} b_{i \bar{\jmath}}^{(P)}+\left(\partial_{\bar{\jmath}} b^{(A)}\right)-b_{\bar{\jmath}}^{(\bar{I})}+i b_{\bar{\jmath}}^{(\bar{H})}=0, \\
& -\frac{i}{2} b_{\bar{\jmath}}^{(\bar{I})}-i b_{\bar{\jmath}}^{(\bar{E})}+4 i \beta^{i} b_{i \bar{\jmath}}^{(P)}+\frac{i}{2} b_{\bar{\jmath}}^{(\bar{I})}+\frac{1}{2} b_{\bar{\jmath}}^{(\bar{H})}+\frac{i}{2} \beta^{i} b_{i \bar{\jmath}}^{(M)}=0, \\
& -\frac{i}{2} b_{j \bar{k}}^{(M)}+\beta^{i} b_{j i \bar{k}}^{(T)}+i b_{j \bar{k}}^{(L)}+\frac{i}{2} b_{i j}^{(N)}\left(\partial_{\bar{k}} \beta^{i}\right)+\frac{i}{2} \beta^{i}\left(\partial_{\bar{k}} b_{i j}^{(N)}\right)-b_{i j \bar{k}}^{(T)} \beta^{i}=0, \\
& -i b_{\bar{\jmath} \bar{k}}^{(\bar{N})}+\beta^{i} b_{\bar{\jmath} i \bar{k}}^{(\bar{T})}-i b_{\bar{\jmath} \bar{k}}^{(\bar{J})}+2 i \beta^{i} b_{\bar{\jmath} \bar{k} i}^{(\bar{S})} \\
& +\frac{i}{2}\left(\partial_{\bar{k}} b_{\bar{\jmath}}^{(\bar{I})}-i \partial_{\bar{k}} b_{\bar{\jmath}}^{(\bar{H})}\right)+\frac{i}{2}\left(\partial_{\bar{k}} \beta^{i}\right) b_{i \bar{\jmath}}^{(M)}+\frac{i}{2}\left(\partial_{\bar{k}} b_{i \bar{\jmath}}^{(M)}\right) \beta^{i}=0, \\
& 4 b_{k j}^{(Q)}+2 \beta^{i} b_{i j k}^{(R)}+2 b_{i k}^{(Q)}\left(\partial_{j} \beta^{i}\right)+\frac{1}{4}\left(\partial_{j} b_{k}^{(\bar{F})}\right) \\
& -\frac{1}{4} b_{k j}^{(K)}-2 \beta^{i}\left(\partial_{j} b_{i k}^{(Q)}\right)-\beta^{i} b_{j k i}^{(R)}=0, \\
& 2 b_{i \bar{\jmath}}^{(P)}+b_{k \bar{\jmath}}^{(P)}\left(\partial_{i} \beta^{k}\right)+\frac{1}{4}\left(\partial_{i} b_{\bar{\jmath}}^{(\bar{E})}\right)-\beta^{k}\left(\partial_{i} b_{k \bar{\jmath}}^{(P)}\right)+\frac{1}{2} b_{i \bar{\jmath}}^{(L)}+\frac{i}{2} b_{k i \bar{\jmath}}^{(T)} \beta^{k}=0, \\
& 2 b_{j k l}^{(R)}+4 \beta^{i} b_{i j k l}^{(V)}+b_{k l i}^{(R)}\left(\partial_{j} \beta^{i}\right)+\frac{1}{4} \partial_{j} b_{k l}^{(K)}-\beta^{i}\left(\partial_{j} b_{k l i}^{(R)}\right)=0, \\
& 2 b_{\bar{\imath} \bar{\jmath} k}^{(\bar{S})}-\frac{i}{2} b_{\{\bar{\imath} \bar{\jmath}\} k}^{(\bar{T})}+2 \beta^{l} b_{l k \bar{\imath} \bar{\jmath}}^{(U)}+b_{\bar{\imath} \jmath}^{(\bar{S})}\left(\partial_{k} \beta^{l}\right)+\frac{1}{4} \partial_{k} b_{\bar{\imath} \bar{\jmath}}^{(\bar{J})} \\
& -\beta^{l}\left(\partial_{k} b_{\bar{\imath} \bar{l} l}^{(\bar{S})}\right)+\frac{1}{2}\left(\partial_{\bar{\imath}} b_{k \bar{\jmath}}^{(L)}\right)+\frac{i}{2} b_{l k \bar{\imath}}^{(T)}\left(\partial_{\bar{\jmath}} \beta^{l}\right)+\frac{i}{2}\left(\partial_{\bar{\jmath}} b_{l k \bar{\imath}}^{(T)}\right) \beta^{l}=0,
\end{aligned}
$$

while consistency equation (8.35c) yields

$$
\begin{aligned}
& \beta^{l}\left(\partial_{l} \bar{b}^{(A)}\right)-\bar{\beta}^{\bar{l}}\left(\partial_{\bar{l}} b^{(A)}\right)=0, \\
& \beta^{l}\left(\partial_{l} \bar{b}^{(\bar{B})}\right)-\bar{\beta}^{\bar{l}}\left(\partial_{\bar{l}} b^{(B)}\right)=0,
\end{aligned}
$$




$$
\begin{aligned}
& -3 \bar{b}^{(\bar{C})}+\beta^{l}\left(\partial_{l} \bar{b}^{(\bar{C})}\right)-3 b^{(C)}-\bar{\beta}^{\bar{l}}\left(\partial_{\bar{l}} b^{(C)}\right)=0, \\
& +2 \bar{b}^{(\bar{D})}+\frac{1}{4}\left(\bar{b}^{(D)}-\bar{b}^{(A)}\right)-\frac{1}{4}\left(\bar{b}^{(B)}+2 \bar{b}^{(\bar{D})}\right)+\beta^{l} \bar{b}_{l}^{(\bar{G})}+\beta^{l} \bar{b}_{l}^{(\bar{E})} \\
& -\frac{i}{4} \beta^{l} \bar{b}_{l}^{(\bar{H})}+\beta^{l}\left(\partial_{l} \bar{b}^{(\bar{D})}\right)+\frac{1}{4}\left(b^{(D)}-b^{(A)}\right)+\frac{i}{4} \bar{\beta}^{\bar{l}} b_{\bar{l}}^{(\bar{H})}-\bar{\beta}^{\bar{l}}\left(\partial_{\bar{l}} b^{(D)}\right)=0, \\
& +2 \bar{b}_{i}^{(\bar{E})}+2 \bar{b}_{i}^{(\bar{G})}+\bar{b}_{i}^{(\bar{I})}-\partial_{k}\left(\frac{1}{4} \bar{b}^{(B)}+2 \bar{b}^{(\bar{D})}\right)-\bar{b}_{i}^{(F)}+2 \beta^{l} \bar{b}_{l i}^{(\bar{J})}+\beta^{l} \partial_{i} \bar{b}_{l}^{(\bar{E})} \\
& +8 \beta^{l} \bar{b}_{l i}^{(\bar{Q})}+\beta^{l}\left(\partial_{l} \bar{b}_{i}^{(\bar{E})}\right)+b_{i}^{(I)}+2 b_{i}^{(E)}-8 \bar{\beta}^{\bar{l}} b_{\bar{l} i}^{(\bar{P})}-\bar{\beta}^{\bar{l}}\left(\partial_{l} b_{i}^{(E)}\right)=0, \\
& -3 \bar{b}_{\bar{\imath}}^{(\bar{F})}+2 i \beta^{l} \bar{b}_{l \bar{\imath}}^{(\bar{O})}+\beta^{l}\left(\partial_{l} \bar{b}_{\bar{\imath}}^{(\bar{F})}\right)-b_{\bar{\imath}}^{(F)} \\
& +\frac{1}{2} \partial_{\bar{\imath}} b^{(C)}-2 \bar{\beta}^{\bar{l}} b_{\bar{l} \bar{\imath}}^{(\bar{K})}-\bar{\beta}^{\bar{l}} \partial_{\bar{\imath}} b_{\bar{l}}^{(F)}-\bar{\beta}^{\bar{l}}\left(\partial_{\bar{l}} b_{\bar{\imath}}^{(F)}\right)=0, \\
& +2 \partial_{i} \bar{b}^{(\bar{D})}+2 \bar{b}_{i}^{(\bar{E})}+2 \bar{b}_{i}^{(\bar{G})}+\bar{b}_{i}^{(\bar{I})}-\partial_{i}\left(\frac{1}{2} \bar{b}^{(B)}+4 \bar{b}^{(\bar{D})}\right)+\beta^{l} \partial_{i} \bar{b}_{l}^{(\bar{G})} \\
& +2 \beta^{l} \bar{b}_{l i}^{(\bar{J})}+2 \beta^{l} \partial_{i} \bar{b}_{l}^{(\bar{E})}+\beta^{l}\left(\partial_{l} \bar{b}_{i}^{(\bar{G})}\right)+\frac{i}{2} b_{i}^{(H)}-b_{i}^{(I)}-b_{i}^{(G)} \\
& +b_{i}^{(E)}-\bar{\beta}^{\bar{l}} b_{\bar{l} i}^{(L)}-4 \bar{\beta}^{\bar{l}} b_{\bar{l} i}^{(P)}-\bar{\beta}^{l}\left(\partial_{\bar{l}} b_{i}^{(G)}\right)=0, \\
& -i \partial_{i}\left(\bar{b}^{(D)}-\bar{b}^{(A)}\right)+\frac{1}{2} \bar{b}_{l}^{(\bar{H})} \partial_{i} \beta^{l}-\frac{1}{2} \beta^{l} \partial_{i} \bar{b}_{l}^{(\bar{H})}+\beta^{l}\left(\partial_{l} \bar{b}_{i}^{(\bar{H})}\right)-b_{i}^{(H)}-2 i b_{i}^{(G)} \\
& -i \partial_{i}\left(b^{(D)}-b^{(A)}\right)-2 i b_{i}^{(E)}-2 i \bar{\beta}^{\bar{l}} b_{\bar{l} i}^{(L)}-4 \bar{\beta}^{\bar{l}} b_{\bar{l} i}^{(\bar{O})}+8 i \bar{\beta}^{\bar{l}} b_{\bar{l} i}^{(P)} \\
& -\frac{1}{2} b_{\bar{l}}^{(\bar{H})} \partial_{i} \bar{\beta}^{\bar{l}}+\frac{1}{2} \bar{\beta}^{\bar{l}} \partial_{i} b_{\bar{l}}^{(\bar{H})}-\bar{\beta}^{\bar{l}}\left(\partial_{\bar{l}} b_{i}^{(H)}\right)=0,
\end{aligned}
$$

$$
\begin{aligned}
- & \frac{1}{2}\left(\bar{b}_{i}^{(\bar{I})}-i \bar{b}_{i}^{(\bar{H})}\right)+\partial_{i} \bar{b}^{(D)}-\frac{1}{2} \beta^{l} \bar{b}_{l i}^{(\bar{N})}+\frac{1}{2} \beta^{l} \partial_{i} \bar{b}_{l}^{(\bar{I})}+\frac{1}{2} \bar{b}_{l}^{(\bar{I})} \partial_{i} \beta^{l}+\beta^{l}\left(\partial_{l} \bar{b}_{i}^{(\bar{I})}\right) \\
& -b_{i}^{(I)}+\frac{1}{2}\left(b_{i}^{(I)}+i b_{i}^{(H)}\right)-2 b_{i}^{(E)}-\partial_{i} b^{(D)}-2 i \bar{\beta}^{\bar{l}} b_{\bar{l} i}^{(\bar{O})}+8 \bar{\beta}^{\bar{l}} b_{\bar{l} i}^{(P)} \\
& +\frac{1}{2} \bar{\beta}^{\bar{l}} b_{\bar{l} i}^{(M)}-\frac{1}{2} \bar{\beta}^{\bar{l}} \partial_{i} b_{\bar{l}}^{(\bar{I})}-\frac{1}{2} b_{\bar{l}}^{(\bar{I})} \partial_{i} \bar{\beta}^{\bar{l}}-\bar{\beta}^{\bar{l}}\left(\partial_{\bar{l}} b_{i}^{(I)}\right)=0,
\end{aligned}
$$

$$
\begin{aligned}
+ & 2 \partial_{j}\left(\bar{b}_{i}^{(\bar{E})}+\bar{b}_{i}^{(\bar{G})}+\frac{1}{2} \bar{b}_{i}^{(\bar{I})}\right)-\frac{1}{4} \partial_{i} \partial_{j} \bar{b}^{(B)}-2 \partial_{i} \partial_{j} \bar{b}^{(\bar{D})}-2 \partial_{i} \bar{b}_{j}^{(F)} \\
& +2 \beta^{l} \partial_{i} \bar{b}_{l j}^{(\bar{J})}+\beta^{l} \partial_{i} \partial_{j} \bar{b}_{l}^{(\bar{E})}+\beta^{l}\left(\partial_{l} \bar{b}_{i j}^{(\bar{J})}\right)+2 b_{i j}^{(N)}+2 b_{i j}^{(J)}-4 \bar{\beta}^{\bar{l}} b_{i j \bar{l}}^{(S)} \\
& +2 i \bar{\beta}^{\bar{l}} b_{i \bar{l} j}^{(T)}-\bar{\beta}^{\bar{l}}\left(\partial_{\bar{l}} b_{i j}^{(J)}\right)=0,
\end{aligned}
$$$$
+4 \bar{b}_{i j}^{(\bar{K})}-2 \partial_{j} \bar{b}_{i}^{(F)}-16 \bar{b}_{i j}^{(\bar{Q})}-\frac{1}{2} \partial_{i} \partial_{j} \bar{b}^{(C)}+2 \beta^{l} \partial_{i} \bar{b}_{l j}^{(\bar{K})}+8 \beta^{l} \bar{b}_{l i j}^{(\bar{R})}
$$$$
+\beta^{l} \partial_{i} \partial_{j} \bar{b}_{l}^{(F)}+16 \beta^{l} \partial_{i} \bar{b}_{l j}^{(\bar{Q})}-4 \beta^{l} \bar{b}_{i j l}^{(\bar{R})}+\beta^{l}\left(\partial_{l} \bar{b}_{i j}^{(\bar{K})}\right)+3 b_{i j}^{(K)}
$$$$
-\bar{\beta}^{\bar{l}}\left(\partial_{\bar{l}} b_{i j}^{(K)}\right)=0 \text {, }
$$ 


$$
\begin{aligned}
& \frac{i}{2} \partial_{i} \bar{b}_{\bar{\jmath}}^{(H)}+\partial_{i} \bar{b}_{\bar{\jmath}}^{(I)}+\partial_{i} \bar{b}_{\bar{\jmath}}^{(G)}+\bar{b}_{i \bar{\jmath}}^{(M)}-2 i \bar{b}_{\bar{\jmath} i}^{(O)}+2 i \bar{b}_{i \bar{\jmath}}^{(\bar{O})}-8 \bar{b}_{i \bar{\jmath}}^{(P)} \\
& -2 \partial_{i} \bar{b}_{\bar{\jmath}}^{(E)}+\beta^{l} \partial_{i} \bar{b}_{l \bar{\jmath}}^{(L)}+8 \beta^{l} \bar{b}_{l i \bar{\jmath}}^{(\bar{S})}-2 i \beta^{l} b_{i l \bar{\jmath}}^{(T)}+8 \beta^{l} \partial_{i} \bar{b}_{l \bar{\jmath}}^{(P)} \\
& +\beta^{l}\left(\partial_{l} \bar{b}_{i \bar{\jmath}}^{(L)}\right)+\frac{i}{2} \partial_{\bar{\jmath}} b_{i}^{(H)}-\partial_{\bar{\jmath}} b_{i}^{(I)}-\partial_{\bar{\jmath}} b_{i}^{(G)}-b_{i \bar{\jmath}}^{(M)}-2 i b_{i \bar{\jmath}}^{(O)} \\
& +2 i b_{\bar{\jmath} i}^{(\bar{O})}+8 b_{i \bar{\jmath}}^{(P)}+2 \partial_{\bar{\jmath}} b_{i}^{(E)}-\bar{\beta}^{\bar{l}} \partial_{\bar{\jmath}} b_{\bar{l} i}^{(L)}-8 \bar{\beta}^{\bar{l}} b_{\bar{l} \bar{l} i}^{(\bar{S})}-2 i \bar{\beta}_{\bar{l}} \bar{b}_{\bar{\jmath} l i}^{(T)} \\
& -8 \bar{\beta}^{\bar{l}} \partial_{\bar{\jmath}} b_{\bar{l} i}^{(P)}-\bar{\beta}^{\bar{l}}\left(\partial_{\bar{l}} b_{\bar{\jmath} i}^{(L)}\right)=0, \\
& \bar{b}_{i \bar{\jmath}}^{(M)}-2 i \bar{b}_{\bar{\jmath} i}^{(\bar{O})}-\frac{1}{2} \partial_{i}\left(\bar{b}_{\bar{\jmath}}^{(I)}-i \bar{b}_{\bar{\jmath}}^{(H)}\right)-\frac{1}{2} \partial_{\bar{\jmath}}\left(\bar{b}_{i}^{(\bar{I})}-i \bar{b}_{i}^{(\bar{H})}\right)+2 \partial_{i} \partial_{\bar{\jmath}} \bar{b}^{(D)} \\
& -2 i \beta^{l} \bar{b}_{i l \bar{\jmath}}^{(\bar{T})}+\frac{1}{2} \bar{b}_{l \bar{\jmath}}^{(M)} \partial_{i} \beta^{l}+\frac{1}{2} \bar{b}_{l i}^{(\bar{N})} \partial_{\bar{\jmath}} \beta^{l}-\frac{1}{2} \beta^{l} \partial_{i} \bar{b}_{l \bar{\jmath}}^{(M)}-\frac{1}{2} \beta^{l} \partial_{\bar{\jmath}} \bar{b}_{l i}^{(\bar{N})} \\
& +\beta^{l} \partial_{i} \partial_{\bar{\jmath}} \bar{b}_{l}^{(\bar{I})}+\bar{b}_{l}^{(\bar{I})} \partial_{i} \partial_{\bar{\jmath}} \beta^{l}+\beta^{l}\left(\partial_{l} \bar{b}_{i \bar{\jmath}}^{(M)}\right)-b_{i \bar{\jmath}}^{(M)}-2 i b_{\bar{\jmath} i}^{(\bar{O})} \\
& +\frac{1}{2} \partial_{i}\left(b_{\bar{\jmath}}^{(I)}+i b_{\bar{\jmath}}^{(H)}\right)+\frac{1}{2} \partial_{\bar{\jmath}}\left(b_{i}^{(\bar{I})}+i b_{i}^{(\bar{H})}\right)-2 \partial_{i} \partial_{\bar{\jmath}} b^{(D)} \\
& -2 i \beta^{l} b_{i l \bar{\jmath}}^{(\bar{T})}-\frac{1}{2} b_{l \bar{\jmath}}^{(M)} \partial_{i} \beta^{l}-\frac{1}{2} b_{l i}^{(\bar{N})} \partial_{\bar{\jmath}} \beta^{l}+\frac{1}{2} \beta^{l} \partial_{i} b_{l \bar{\jmath}}^{(M)}+\frac{1}{2} \beta^{l} \partial_{\bar{\jmath}} b_{l i}^{(\bar{N})} \\
& -\beta^{l} \partial_{i} \partial_{\bar{\jmath}} b_{l}^{(\bar{I})}-b_{l}^{(\bar{I})} \partial_{i} \partial_{\bar{\jmath}} \beta^{l}-\beta^{l}\left(\partial_{l} b_{i \bar{\jmath}}^{(M)}\right)=0, \\
& -\frac{1}{2} \partial_{j}\left(\bar{b}_{i}^{(\bar{I})}-i \bar{b}_{i}^{(\bar{H})}\right)+\partial_{i} \partial_{j} \bar{b}^{(\bar{D})}+\frac{1}{2} \bar{b}_{l j}^{(\bar{N})} \partial_{i} \beta^{l}-\frac{1}{2} \beta^{l} \partial_{i} \bar{b}_{l j}^{(\bar{N})} \\
& +\frac{1}{2} \beta^{l} \partial_{i} \partial_{j} \bar{b}_{l}^{(\bar{I})}+\frac{1}{2} \bar{b}_{l}^{(\bar{I})} \partial_{i} \partial_{j} \beta^{l}+\beta^{l}\left(\partial_{l} \bar{b}_{i j}^{(\bar{N})}\right)-2 b_{i j}^{(N)}+\frac{1}{2} \partial_{j}\left(b_{i}^{(I)}+i b_{i}^{(H)}\right) \\
& -b_{i j}^{(J)}-\partial_{i} \partial_{j} b^{(D)}-2 i \bar{\beta}^{\bar{l}} b_{i \bar{l} j}^{(T)}+4 \bar{\beta}^{\bar{l}} b_{i j \bar{l}}^{(S)}-\frac{1}{2} b_{\bar{l} j}^{(M)} \partial_{i} \bar{\beta}^{\bar{l}}+\frac{1}{2} \bar{\beta}^{\bar{l}} \partial_{i} b_{\bar{l} j}^{(M)} \\
& -\frac{1}{2} \bar{\beta}^{\bar{l}} \partial_{i} \partial_{j} b_{\bar{l}}^{(\bar{I})}-\frac{1}{2} b_{\bar{l}}^{(\bar{I})} \partial_{i} \partial_{j} \bar{\beta}^{\bar{l}}-\bar{\beta}^{\bar{l}}\left(\partial_{\bar{l}} b_{i j}^{(N)}\right)=0,
\end{aligned}
$$

$$
\begin{aligned}
& \frac{i}{2} \partial_{i} \bar{b}_{\bar{\jmath}}^{(I)}+2 \bar{b}_{i \bar{\jmath}}^{(\bar{O})}+8 i \bar{b}_{i \bar{\jmath}}^{(P)}-\frac{1}{2}\left(\frac{1}{2} \bar{b}_{i \bar{\jmath}}^{(\bar{O})}-\frac{1}{2} \bar{b}_{i \bar{\jmath}}^{(O)}-i \bar{b}_{i \bar{\jmath}}^{(L)}\right)+2 i \partial_{i} \bar{b}_{\bar{\jmath}}^{(E)} \\
& \quad+\beta^{l} \partial_{i} \bar{b}_{l \bar{\jmath}}^{(\bar{O})}-8 i \beta^{l} \bar{b}_{l i \bar{\jmath}}^{(\bar{S})}-8 i \beta^{l} \partial_{i} b_{l \bar{\jmath}}^{(P)}-\frac{1}{2} \beta^{l} \bar{b}_{l i \bar{\jmath}}^{(\bar{T})}+\beta^{l}\left(\partial_{l} \bar{b}_{i \bar{\jmath}}^{(\bar{O})}\right)+\frac{i}{2} b_{\bar{\jmath} i}^{(M)} \\
& \quad-b_{i \bar{\jmath}}^{(O)}+\frac{1}{2}\left(\frac{1}{2} b_{\bar{\jmath} i}^{(\bar{O})}-\frac{1}{2} b_{\bar{\jmath} i}^{(O)}+i b_{\bar{\jmath} i}^{(L)}\right)-\bar{\beta}^{\bar{l}} b_{\bar{\jmath} \bar{l} i}^{(\bar{T})}+\frac{1}{2} \bar{\beta}^{\bar{l}} b_{\bar{l} \bar{i} i}^{(\bar{T})}+\bar{\beta}^{\bar{l}}\left(\partial_{l} b_{i \bar{\jmath}}^{(O)}\right)=0,
\end{aligned}
$$

$$
\begin{aligned}
& \frac{i}{2} \bar{b}_{i \bar{\jmath}}^{(\bar{O})}-2 \bar{b}_{i \bar{\jmath}}^{(P)}-\frac{1}{4} \partial_{i} \bar{b}_{\bar{\jmath}}^{(E)}+2 \beta^{l} \bar{b}_{l i \bar{\jmath}}^{(\bar{S})}+\beta^{l} \partial_{i} \bar{b}_{l \bar{\jmath}}^{(P)}+\beta^{l}\left(\partial_{l} \bar{b}_{i \bar{\jmath}}^{(P)}\right) \\
& \quad+\frac{i}{2} b_{i \bar{\jmath}}^{(\bar{O})}+2 b_{i \bar{\jmath}}^{(P)}+\frac{1}{4} \partial_{\bar{\jmath}} b_{i}^{(E)}-2 \bar{\beta}^{\bar{l}} b_{\bar{l} \bar{\jmath} i}^{(\bar{S})}-\bar{\beta}^{\bar{l}} \partial_{\bar{\jmath}} b_{\bar{l} i}^{(P)}-\bar{\beta}^{\bar{l}}\left(\partial_{\bar{l}} b_{i \bar{\jmath}}^{(P)}\right)=0,
\end{aligned}
$$

$$
\begin{aligned}
& -\bar{b}_{i j}^{(\bar{Q})}-\frac{1}{4} \partial_{i} \bar{b}_{j}^{(F)}-\frac{1}{4} \bar{b}_{i j}^{(\bar{K})}+2 \beta^{l} \bar{b}_{l i j}^{(\bar{R})}+2 \beta^{l} \partial_{i} \bar{b}_{l j}^{(\bar{Q})} \\
& \quad+\beta^{l} \bar{b}_{i j l}^{(\bar{R})}+\beta^{l}\left(\partial_{l} \bar{b}_{i j}^{(\bar{Q})}\right)+3 b_{i j}^{(Q)}-\bar{\beta}^{\bar{l}}\left(\partial_{l} b_{i j}^{(Q)}\right)=0,
\end{aligned}
$$




$$
\begin{aligned}
+ & 3 \bar{b}_{i j k}^{(\bar{R})}-4 \partial_{i} \bar{b}_{j k}^{(\bar{Q})}-\frac{1}{4} \partial_{k} \bar{b}_{i j}^{(\bar{K})}+\frac{1}{2} \partial_{i} \bar{b}_{k j}^{(\bar{K})}+2 \beta^{l} \partial_{i} \bar{b}_{l j k}^{(\bar{R})} \\
& +4 \beta^{l}\left(\bar{b}_{l k i j}^{(\bar{V})}+\bar{b}_{l i k j}^{(\bar{V})}\right)+2 \beta^{l} \partial_{i} \partial_{j} \bar{b}_{l k}^{(\bar{Q})}+\beta^{l} \partial_{k} \bar{b}_{i j l}^{(\bar{R})}-2 \beta^{l} \partial_{i} \bar{b}_{j k l}^{(\bar{R})} \\
& +\beta^{l}\left(\partial_{l} \bar{b}_{i j k}^{(\bar{R})}\right)+3 b_{i j k}^{(R)}-\bar{\beta}^{\bar{l}}\left(\partial_{l} b_{i j k}^{(R)}\right)=0,
\end{aligned}
$$

$$
\begin{aligned}
& \partial_{i}\left(\frac{i}{2} \bar{b}_{j \bar{k}}^{(\bar{O})}-2 \bar{b}_{j \bar{k}}^{(P)}\right)-\frac{1}{4} \partial_{i} \partial_{j} \bar{b}_{\bar{k}}^{(E)}+2 \beta^{l} \partial_{i} \bar{b}_{l j \bar{k}}^{(\bar{S})}+\beta^{l} \partial_{i} \partial_{j} \bar{b}_{l \bar{k}}^{(P)} \\
& \quad+\beta^{l}\left(\partial_{l} \bar{b}_{i j \bar{k}}^{(\bar{S})}\right)+2 b_{i j \bar{k}}^{(S)}+\frac{i}{2} b_{i j \bar{k}}^{(T)}+\frac{1}{4} \partial_{i} \partial_{j} b_{\bar{k}}^{(F)}+\frac{1}{4} \partial_{\bar{k}} b_{i j}^{(J)}-2 \bar{\beta}^{\bar{l}} b_{i j \bar{k} \bar{l}}^{(U)} \\
& \quad-\bar{\beta}^{\bar{l}}\left(\partial_{\bar{k}} b_{i j \bar{l}}^{(S)}\right)-\bar{\beta}^{\bar{l}}\left(\partial_{\bar{l}} b_{i j \bar{k}}^{(S)}\right)=0
\end{aligned}
$$

$$
\begin{aligned}
& \frac{i}{2} \partial_{j} \bar{b}_{i \bar{k}}^{(M)}+\partial_{j} \bar{b}_{\bar{k} i}^{(O)}-\frac{1}{2} \partial_{i}\left(\frac{1}{2} \bar{b}_{j \bar{k}}^{(\bar{O})}-\frac{1}{2} \bar{b}_{j \bar{k}}^{(O)}-i \bar{b}_{j \bar{k}}^{(L)}\right)+\beta^{l} \partial_{j} \bar{b}_{i l \bar{k}}^{(\bar{T})} \\
& \quad+\frac{1}{2} \bar{b}_{l j \bar{k}}^{(\bar{T})} \partial_{i} \beta^{l}-\frac{1}{2} \beta^{l} \partial_{i} \bar{b}_{l j \bar{k}}^{(\bar{T})}+\beta^{l}\left(\partial_{l} \bar{b}_{i j \bar{k}}^{(\bar{T})}\right)+i \partial_{\bar{k}} b_{i j}^{(N)}+8 i b_{j i \bar{k}}^{(S)} \\
& \quad-2 b_{j i \bar{k}}^{(T)}+\frac{1}{2} \partial_{i}\left(\frac{1}{2} b_{\bar{k} j}^{(\bar{O})}-\frac{1}{2} b_{\bar{k} j}^{(O)}+i b_{\bar{k} j}^{(L)}\right)+2 i \partial_{\bar{k}} b_{i j}^{(J)}-\bar{\beta}^{\bar{l}} \partial_{\bar{k}} b_{i \bar{l} j}^{(T)} \\
& \quad-8 i \bar{\beta}^{\bar{l}} b_{i j \bar{k} \bar{l}}^{(U)}-8 i \bar{\beta}^{\bar{l}} \partial_{\bar{k}} b_{i j \bar{l}}^{(S)}-\frac{1}{2} b_{\bar{l} j j}^{(\bar{T})} \partial_{i} \bar{\beta}^{\bar{l}}+\frac{1}{2} \bar{\beta}^{\bar{l}} \partial_{i} b_{\overline{l k} j}^{(\bar{T})}-\bar{\beta}^{\bar{l}}\left(\partial_{\bar{l}} b_{i j \bar{k}}^{(T)}\right)=0,
\end{aligned}
$$

$$
\begin{aligned}
& \partial_{i}\left(-2 \bar{b}_{\bar{k} \bar{l} j}^{(S)}+\frac{i}{2} \bar{b}_{\bar{k} \bar{l} j}^{(T)}\right)-\frac{1}{4} \partial_{i} \partial_{j} \bar{b}_{\bar{k} \bar{l}}^{(J)}+2 \beta^{m} \partial_{i} \bar{b}_{m j \bar{k} \bar{l}}^{(U)}+\beta^{m} \partial_{i} \partial_{j} \bar{b}_{\bar{k} \bar{l} m}^{(S)} \\
& \quad+\beta^{m}\left(\partial_{m} \bar{b}_{i j \bar{k} \bar{l}}^{(U)}\right)-\partial_{\bar{k}}\left(-2 b_{i j \bar{l}}^{(S)}-\frac{i}{2} b_{i j \bar{l}}^{(T)}\right)+\frac{1}{4} \partial_{\bar{k}} \partial_{\bar{l}} b_{i j}^{(J)}-2 \bar{\beta}^{\bar{m}} \partial_{\bar{k}} b_{\bar{m} \bar{l} j}^{(U)} \\
& \quad-\bar{\beta}^{\bar{m}} \partial_{\bar{k}} \partial_{\bar{l}} b_{i j \bar{m}}^{(S)}-\bar{\beta}^{\bar{m}}\left(\partial_{\bar{m}} b_{i j \bar{k}}^{(U)}\right)=0, \\
& 3 \bar{b}_{i j k l}^{(\bar{V})}-2 \partial_{i} \bar{b}_{j k l}^{(\bar{R})}-\frac{1}{4} \partial_{i} \partial_{j} \bar{b}_{k l}^{(\bar{K})}+\beta^{m} \partial_{i} \bar{b}_{m j k l}^{(\bar{V})} \\
& \quad+\beta^{m} \partial_{i} \partial_{j} \bar{b}_{k l m}^{(\bar{R})}+\beta^{m}\left(\partial_{m} \bar{b}_{i j k l}^{(\bar{V})}\right)+3 b_{i j k l}^{(V)}-\bar{\beta}^{\bar{m}}\left(\partial_{\bar{m}} b_{i j k l}^{(V)}\right)=0 .
\end{aligned}
$$





\section{Appendix E}

\section{Minimal Algebra on Chiral Fields}

The following follows from the superalgebra for a chiral $(\lambda)$ or antichiral $(\bar{\lambda})$ scalar superfield. Although trivial, these special cases occur sufficiently frequent to earn explicit treatment,

$$
\begin{aligned}
\mathcal{D}^{2} \overline{\mathcal{D}}^{2} \bar{\lambda} & =\left(8 i G_{\alpha \dot{\alpha}} \mathcal{D}^{\alpha \dot{\alpha}}-8 \mathcal{D}_{\alpha \dot{\alpha}} \mathcal{D}^{\alpha \dot{\alpha}}+4\left(\overline{\mathcal{D}}_{\dot{\alpha}} \bar{R}\right) \overline{\mathcal{D}}^{\dot{\alpha}}+8 \bar{R} \overline{\mathcal{D}}^{2}\right) \bar{\lambda} \\
\mathcal{D}^{\alpha} \mathcal{D}_{\alpha \dot{\alpha}} \lambda & =\mathcal{D}_{\alpha \dot{\alpha}} \mathcal{D}^{\alpha} \lambda-2 i G_{\alpha \dot{\alpha}} \mathcal{D}^{\alpha} \lambda \\
\mathcal{D}^{\alpha} \mathcal{D}_{\alpha \dot{\alpha}} \bar{\lambda} & =2 i \bar{R} \overline{\mathcal{D}}_{\dot{\alpha}} \bar{\lambda} \\
\left(\mathcal{D}_{\alpha} \overline{\mathcal{D}}^{2} \bar{\lambda}\right) & =4\left(G_{\alpha \dot{\alpha}}-i \mathcal{D}_{\alpha \dot{\alpha}}\right)\left(\overline{\mathcal{D}}^{\dot{\alpha}} \bar{\lambda}\right) \\
\left(\mathcal{D}^{\alpha} \mathcal{D}^{2} \lambda\right) & =4 \bar{R}\left(\mathcal{D}^{\alpha} \lambda\right) \\
\left(\mathcal{D}^{2} \mathcal{D}_{\alpha} \lambda\right) & =-2 \bar{R}\left(\mathcal{D}_{\alpha} \lambda\right) \\
\left(\mathcal{D}^{2} \overline{\mathcal{D}}_{\dot{\alpha}} \bar{\lambda}\right) & =4 \bar{R}\left(\overline{\mathcal{D}}_{\dot{\alpha}} \bar{\lambda}\right) \\
\mathcal{D}_{\alpha}\left(\mathcal{D}^{\beta} \lambda\right)\left(\mathcal{D}_{\beta} \lambda\right) & =-\left(\mathcal{D}_{\alpha} \lambda\right)\left(\mathcal{D}^{2} \lambda\right) \\
\left(\overline{\mathcal{D}}^{\dot{\alpha}} \mathcal{D}_{\alpha \dot{\alpha}} \bar{\lambda}\right) & =\left(\mathcal{D}_{\alpha \dot{\alpha}} \overline{\mathcal{D}}^{\dot{\alpha}} \bar{\lambda}\right)-2 i G_{\alpha}^{\dot{\alpha}}\left(\overline{\mathcal{D}}_{\dot{\alpha}} \bar{\lambda}\right) \\
\left(\overline{\mathcal{D}}^{\dot{\alpha}} \mathcal{D}_{\alpha \dot{\alpha}} \lambda\right) & =-2 i R\left(\mathcal{D}_{\alpha} \lambda\right)
\end{aligned}
$$




$$
\begin{aligned}
\left(\overline{\mathcal{D}}_{\dot{\alpha}} \mathcal{D}^{2} \lambda\right) & =4\left(G_{\alpha \dot{\alpha}}+i \mathcal{D}_{\alpha \dot{\alpha}}\right)\left(\mathcal{D}^{\alpha} \lambda\right), \\
\left(\mathcal{D}_{\alpha} \mathcal{D}_{\beta} \lambda\right) & =\frac{1}{2} \varepsilon_{\alpha \beta}\left(\mathcal{D}^{2} \lambda\right) \\
\left(\mathcal{D}^{\alpha} \mathcal{D}_{\alpha \dot{\alpha}} \overline{\mathcal{D}}^{\dot{\alpha}} \bar{\lambda}\right) & =-2 i \mathcal{D}^{\alpha \dot{\alpha}} \mathcal{D}_{\alpha \dot{\alpha}} \bar{\lambda}+2 i \bar{R} \overline{\mathcal{D}}^{2} \bar{\lambda}+4 G_{\alpha \dot{\alpha}} \mathcal{D}^{\alpha \dot{\alpha}} \bar{\lambda}
\end{aligned}
$$

Weyl variations for derivatives acting on chiral fields of Weyl weight 0 are given by

$$
\begin{aligned}
\delta^{\prime}[\lambda]= & 0, \\
\delta^{\prime}\left[\mathcal{D}^{\alpha} \lambda\right]= & \left(\frac{1}{2} \sigma^{\prime}-\bar{\sigma}^{\prime}\right) \mathcal{D}^{\alpha} \lambda, \\
\delta^{\prime}\left[\mathcal{D}_{\alpha \dot{\alpha}} \lambda\right]= & -\frac{1}{2}\left(\sigma^{\prime}+\bar{\sigma}^{\prime}\right) \mathcal{D}_{\alpha \dot{\alpha}} \lambda-\frac{i}{2}\left(\overline{\mathcal{D}}_{\dot{\alpha}} \bar{\sigma}^{\prime}\right) \mathcal{D}_{\alpha} \lambda, \\
\delta^{\prime}\left[\mathcal{D}^{2} \lambda\right]= & \left(\sigma^{\prime}-2 \bar{\sigma}^{\prime}\right) \mathcal{D}^{2} \lambda+2\left(\mathcal{D}^{\alpha} \sigma^{\prime}\right) \mathcal{D}_{\alpha} \lambda, \\
\delta^{\prime}\left[\overline{\mathcal{D}}^{2} \bar{\lambda}\right]= & \left(\bar{\sigma}^{\prime}-2 \sigma^{\prime}\right) \overline{\mathcal{D}}^{2} \bar{\lambda}+2\left(\overline{\mathcal{D}}_{\dot{\alpha}} \bar{\sigma}^{\prime}\right) \overline{\mathcal{D}}^{\dot{\alpha}} \bar{\lambda} \\
\delta^{\prime}\left[\mathcal{D}_{\alpha \dot{\alpha}} \bar{\lambda}\right]= & -\frac{1}{2}\left(\sigma^{\prime}+\bar{\sigma}^{\prime}\right) \mathcal{D}_{\alpha \dot{\alpha}} \bar{\lambda}-\frac{i}{2}\left(\mathcal{D}_{\alpha} \sigma^{\prime}\right) \overline{\mathcal{D}} \dot{\alpha} \bar{\lambda} \\
\delta^{\prime}\left[\mathcal{D}^{\alpha \dot{\alpha}} \overline{\mathcal{D}}_{\dot{\alpha}} \bar{\lambda}\right]= & -\frac{3}{2} \sigma^{\prime} \mathcal{D}^{\alpha \dot{\alpha}} \overline{\mathcal{D}}_{\dot{\alpha}} \bar{\lambda}+\frac{1}{2}\left(\mathcal{D}^{\alpha \dot{\alpha}} \sigma^{\prime}\right) \overline{\mathcal{D}}_{\dot{\alpha}} \bar{\lambda} \\
& +\frac{i}{2}\left(\mathcal{D}^{\alpha} \sigma^{\prime}\right) \overline{\mathcal{D}}^{2} \bar{\lambda}+\left(\overline{\mathcal{D}}_{\dot{\alpha}} \bar{\sigma}^{\prime}\right) \mathcal{D}^{\alpha \dot{\alpha}} \bar{\lambda} \\
& +\frac{1}{2}\left(\mathcal{D}^{\alpha \dot{\alpha}} \bar{\sigma}^{\prime}\right) \overline{\mathcal{D}}_{\dot{\alpha}} \bar{\lambda} \\
\delta^{\prime}\left[\mathcal{D}^{\alpha \dot{\alpha}} \mathcal{D}_{\alpha} \lambda\right]= & -\frac{3}{2} \bar{\sigma}^{\prime} \mathcal{D}^{\alpha \dot{\alpha}} \mathcal{D}_{\alpha} \lambda+\frac{1}{2}\left(\mathcal{D}^{\alpha \dot{\alpha}} \bar{\sigma}^{\prime}\right) \mathcal{D}_{\alpha} \lambda \\
& -\frac{i}{2}\left(\overline{\mathcal{D}}^{\dot{\alpha}} \bar{\sigma}^{\prime}\right) \mathcal{D}^{2} \lambda+\left(\mathcal{D}_{\alpha} \sigma^{\prime}\right) \mathcal{D}^{\alpha \dot{\alpha}} \lambda \\
& +\frac{1}{2}\left(\mathcal{D}^{\alpha \dot{\alpha}} \sigma^{\prime}\right) \mathcal{D}_{\alpha} \lambda .
\end{aligned}
$$




\section{Bibliography}

[1] J. Erdmenger, J. Grosse and Z. Guralnik. Spectral flow on the Higgs branch and AdS/CFT duality. JHEP 06:052 (2005). hepth/0502224. xix, xxvii

[2] J. Erdmenger, N. Evans and J. Grosse. Heavy-light mesons from the AdS/CFT correspondence (2006). hep-th/0605241. xix, 75

[3] R. Apreda, J. Erdmenger, N. Evans, J. Grosse and Z. GuRALNIK. Instantons on D7 Brane Probes and AdS/CFT with Flavour. Fortsch. Phys. 54:266-274 (2006). hep-th/0601130. xix

[4] J. Grosse. MathPSfrag: Creating Publication-Quality Labels in Mathematica Plots (2005). cs.GR/0510087. xix

[5] G. 'T Hooft. A planar diagram theory for string interactions. Nucl. Phys. B72:461 (1974). xxiii, 19

[6] R. Gopakumar. From free fields to AdS. Phys. Rev. D70:025009 (2004). hep-th/0308184. xxiv

[7] J. M. Maldacena. The large $N$ limit of superconformal field theories and supergravity. Adv. Theor. Math. Phys. 2:231-252 (1998). hep-th/9711200. xxiv, 55

[8] S. S. Gubser, I. R. Klebanov and A. M. Polyakov. Gauge 
theory correlators from non-critical string theory. Phys. Lett. B428:105-114 (1998). hep-th/9802109. xxv, 55

[9] E. Witten. Anti-de Sitter space and holography. Adv. Theor. Math. Phys. 2:253-291 (1998). hep-th/9802150. xxv, 55

[10] D. Z. Freedman, S. S. Gubser, K. Pilch and N. P. Warner. Renormalization group flows from holography supersymmetry and a c-theorem. Adv. Theor. Math. Phys. 3:363-417 (1999). hepth/9904017. xxv, xxvii, 17, 129

[11] S.-M. Lee, S. Minwalla, M. Rangamani and N. Seiberg. Three-point Functions of Chiral Operators in $D=4, \mathcal{N}=4$ SYM at large N. Adv. Theor. Math. Phys. 2:697-718 (1998). hepth/9806074. xxv

[12] N. N. Bogolyubov and D. V. Shirkov. Introduction to the Theory of Quantized Fields. Intersci. Monogr. Phys. Astron. 3:1720 (1959). xxv

[13] C. Becchi. Current Algebra Ward Identities in the Renormalized $\sigma$ Model. Comm. Math. Phys. 39(4):329-344 (1974). [euclid.cmp/1103860236]. xxv

[14] H. Epstein and V. Glaser. Adiabatic Limit in Perturbation Theory (1975). In Erice 1975, Proceedings, Renormalization Theory, Dordrecht 1976, 193-254 [CERN-TH-1344]. xxv

[15] M. Henningson and K. Skenderis. The holographic Weyl anomaly. JHEP 07:023 (1998). hep-th/9806087. xxvii

[16] E. T. Akhmedov. A Remark on the AdS/CFT Correspondence and the Renormalization Group Flow. Phys. Lett. B442:152-158 (1998). hep-th/9806217. xxvii

[17] A. Karch, D. Lüst and A. Miemiec. New $\mathcal{N}=1$ superconformal field theories and their supergravity description. Phys. Lett. B454:265-269 (1999). hep-th/9901041. xxvii, 17 
[18] A. Fayyazuddin and M. Spalinski. Large $N$ superconformal gauge theories and supergravity orientifolds. Nucl. Phys. B535:219232 (1998). hep-th/9805096. xxvii, 19

[19] O. Aharony, A. Fayyazuddin and J. M. Maldacena. The Large $N$ Limit of $\mathcal{N}=2,1$ Field Theories from Three-Branes in F-theory. JHEP 07:013 (1998). hep-th/9806159. xxvii, 19

[20] A. KARCh and E. KATZ. Adding flavor to AdS/CFT. JHEP 06:043 (2002). hep-th/0205236. xxvii, 19, 20, 21, 23, 55, 58, 75, 155

[21] P. Breitenlohner and D. Z. Freedman. Stability in gauged extended supergravity. Ann. Phys. 144:249 (1982). xxvii, 45, 58

[22] M. Bertolini, P. Di Vecchia, M. Frau, A. Lerda and R. Marotta. $\mathcal{N}=2$ gauge theories on systems of fractional D3/D7 branes. Nucl. Phys. B621:157-178 (2002). hep-th/0107057. xxvii

[23] T. Sakai and J. Sonnenschein. Probing flavored mesons of confining gauge theories by supergravity. JHEP 09:047 (2003). hepth/0305049. xxvii

[24] M. Kruczenski, D. Mateos, R. C. Myers and D. J. Winters. Meson spectroscopy in AdS/CFT with flavour. JHEP 07:049 (2003). hep-th/0304032. xxvii, xxviii, 23, 25, 39, 40, 55, 69, 75, 155

[25] M. Graña and J. Polchinski. Gauge / gravity duals with holomorphic dilaton. Phys. Rev. D65:126005 (2002). hep-th/0106014. xxvii

[26] R. C. Myers and R. M. Thomson. Holographic mesons in various dimensions (2006). hep-th/0605017. xxvii

[27] I. KIRSCH and D. VAman. The D3/D7 background and flavor dependence of Regge trajectories. Phys. Rev. D72:026007 (2005). hep-th/0505164. xxvii

[28] D. Arean and A. V. Ramallo. Open String Modes at Brane Intersections. JHEP 04:037 (2006). hep-th/0602174. xxvii 
[29] C. Núñez, A. Paredes and A. V. Ramallo. Flavoring the gravity dual of $\mathcal{N}=1$ Yang-Mills with probes. JHEP 12:024 (2003). hep-th/0311201. xxvii

[30] X.-J. WANG and S. Hu. Intersecting branes and adding flavors to the Maldacena-Núñez background. JHEP 09:017 (2003). hepth/0307218. xxvii

[31] R. Casero, C. Núñez and A. Paredes. Towards the string dual of $\mathcal{N}=1$ SQCD-like theories. Phys. Rev. D73:086005 (2006). hepth/0602027. xxvii

[32] J. D. Edelstein and R. Portugues. Gauge / string duality in confining theories (2006). hep-th/0602021. xxvii

[33] R. Casero, A. Paredes and J. Sonnenschein. Fundamental matter, meson spectroscopy and non-critical string / gauge duality. JHEP 01:127 (2006). hep-th/0510110. xxvii

[34] T. Hirayama. A holographic dual of CFT with flavor on de Sitter space (2006). hep-th/0602258. xxvii

[35] R. A. Janik and R. Peschanski. Asymptotic perfect fluid dynamics as a consequence of AdS/CFT. Phys. Rev. D73:045013 (2006). hep-th/0512162. xxvii

[36] F. Canoura, J. D. Edelstein, L. A. P. Zayas, A. V. RaMALlo and D. VAman. Supersymmetric branes on $\operatorname{AdS}_{\times} Y^{*}(p, q)$ and their field theory duals. JHEP 03:101 (2006). hep-th/0512087. xxvii

[37] S. Benvenuti, M. Mahato, L. A. Pando Zayas and Y. TACHIKAWA. The gauge / gravity theory of blown up four cycles (2005). hep-th/0512061. xxvii

[38] S. S. GubSER. Dilaton-driven confinement (1999). hep-th/9902155. xxviii, xxxi, 32, 40, 41, 156

[39] E. SchreIBer. Excited Mesons and Quantization of String Endpoints (2004). hep-th/0403226. xxix, 48, 51 
[40] L. Y. Glozman. Chiral and $\mathrm{U}(1)_{A}$ Restorations High in the Hadron Spectrum, Semiclassical Approximation and Large $N_{c}$. Int. J. Mod. Phys. A21:475-486 (2006). hep-ph/0411281. xxix, 48

[41] M. Shifman. Highly Excited Hadrons in QCD and Beyond (2005). hep-ph/0507246. xxix, 51

[42] N. R. Constable and R. C. Myers. Exotic scalar states in the AdS/CFT correspondence. JHEP 11:020 (1999). hep-th/9905081. xxxi, 32, 85, 157

[43] A. B. Zamolodchikov. 'Irreversibility' of the Flux of the Renormalization Group in a 2-D Field Theory. JETP Lett. 43:730-732 (1986). xxxii, 128

[44] D. Anselmi, D. Z. Freedman, M. T. Grisaru and A. A. JoHANSEn. Nonperturbative Formulas for Central Functions of Supersymmetric Gauge Theories. Nucl. Phys. B526:543-571 (1998). hep-th/9708042. xxxiii

[45] D. Anselmi, J. Erlich, D. Z. Freedman and A. A. Johansen. Positivity Constraints on Anomalies in Supersymmetric Gauge Theories. Phys. Rev. D57:7570-7588 (1998). hep-th/9711035. xxxiii

[46] J. L. CARDy. Is there a $c$ Theorem in Four Dimensions? Phys. Lett. B215:749-752 (1988). xxxiii, 129

[47] K. Intriligator and B. Wecht. The Exact Superconformal RSymmetry Maximizes a. Nucl. Phys. B667:183-200 (2003). hepth/0304128. xxxiii

[48] H. Osborn. Weyl consistency conditions and a local renormalization group equation for general renormalizable field theories. Nucl. Phys. B363:486-526 (1991). xxxiii, 95, 133, 159

[49] H. Osborn. Local couplings and $\mathrm{Sl}(2, \mathbb{R})$ invariance for gauge theories at one loop. Phys. Lett. B561:174-182 (2003). hep-th/0302119. xxxiv, 147, 159 
[50] R. J. Riegert. A nonlocal action for the trace anomaly. Phys. Lett. B134:56-60 (1984). xxxv, 126

[51] E. S. Fradkin and A. A. Tseytlin. Conformal Supergravity. Phys. Rept. 119:233-362 (1985). xxxv, 148

[52] R. P. Manvelyan. Superweyl cocycle in $d=4$ and superconformal-invariant operator. Phys. Lett. B373:306-308 (1996). hepth/9512045. xxxv, 150

[53] B. DE Wit and M. T. Grisaru. Compensating Fields and Anomalies (1985). In 'Quantum Field Theory and Quantum Statistics: Essays in Honor of 60th birthday of E.S. Fradkin. xxxv, 161

[54] Y. Shamir. Compensating Fields and Anomalies in Supergravity. Nucl. Phys. B389:323-348 (1993). hep-th/9207038. xxxv, 161

[55] J. D. Buorken. Asymptotic Sum Rules at Infinite Momentum. Phys. Rev. 179:1547-1553 (1969). 3

[56] R. P. Feynman. Photon-hadron Interactions (1972). Reading 1972, 282p. 3

[57] S. Eidelman et. al. (Particle Data Group). Review of Particle Physics. Phys. Lett. B 592:1+ (2004). 4

[58] M. F. Sohnius and P. C. West. Conformal Invariance in $\mathcal{N}=4$ Supersymmetric Yang-Mills Theory. Phys. Lett. B100:245 (1981). 5

[59] E. D'Hoker and D. Z. Freedman. Supersymmetric gauge theories and the AdS/CFT correspondence (2002). hep-th/0201253. 7

[60] G. Dall'Agata, K. Lechner and M. Tonin. Action for IIB supergravity in 10 dimensions (1998). hep-th/9812170. 7

[61] G. Dall'Agata, K. Lechner and M. Tonin. $D=10, \mathcal{N}=I I B$ supergravity: Lorentz-invariant actions and duality. JHEP 07:017 (1998). hep-th/9806140. 7 
[62] J. Polchinski. String theory. Vol. 2: Superstring theory and beyond (1998). Cambridge, UK: Univ. Pr. (1998) 531 p. 7

[63] M. B. Green, J. H. Schwarz and E. Witten. Superstring Theory. Vol. 2: Loop Amplitudes, Anomalies and Phenomenology (1987). Cambridge, Uk: Univ. Pr. ( 1987) 596 P. (Cambridge Monographs On Mathematical Physics). 8

[64] M. B. Green and J. H. Schwarz. Extended Supergravity in Ten Dimensions. Phys. Lett. B122:143 (1983). 8

[65] P. S. Howe and P. C. West. The Complete $\mathcal{N}=2, d=10$ Supergravity. Nucl. Phys. B238:181 (1984). 8

[66] J. H. Schwarz and P. C. West. Symmetries and Transformations of Chiral $\mathcal{N}=2 D=10$ Supergravity. Phys. Lett. B126:301 (1983). 8

[67] J. H. Schwarz. Covariant Field Equations of Chiral $\mathcal{N}=2 D=10$ Supergravity. Nucl. Phys. B226:269 (1983). 8

[68] J. Polchinski, S. Chaudhuri and C. V. Johnson. Notes on D-Branes (1996). hep-th/9602052. 9

[69] J. Polchinski. Dirichlet-Branes and Ramond-Ramond Charges. Phys. Rev. Lett. 75:4724-4727 (1995). hep-th/9510017. 9

[70] A. Giveon, M. Porrati and E. Rabinovici. Target space duality in string theory. Phys. Rept. 244:77-202 (1994). hep-th/9401139. 9

[71] K. Kikkawa and M. Yamasaki. Casimir Effects in Superstring Theories. Phys. Lett. B149:357 (1984). 9

[72] E. Alvarez, L. Alvarez-Gaume, J. L. F. Barbon and Y. Lozano. Some Global Aspects of Duality in String Theory. Nucl. Phys. B415:71-100 (1994). hep-th/9309039. 9

[73] J. Dai, R. G. Leigh and J. Polchinski. New Connection between String Theories. Mod. Phys. Lett. A4:2073-2083 (1989). 9 
[74] J. E. Paton and H.-M. Chan. Generalized Veneziano Model with Isospin. Nucl. Phys. B10:516-520 (1969). 10

[75] R. G. Leigh. Dirac-Born-Infeld Action from Dirichlet Sigma Model. Mod. Phys. Lett. A4:2767 (1989). 10

[76] R. C. Myers. Dielectric-branes. JHEP 12:022 (1999). hepth/9910053. 11

[77] A. A. Tseytuin. On non-abelian generalisation of the Born-Infeld action in string theory. Nucl. Phys. B501:41-52 (1997). hepth/9701125. 12

[78] M. R. Garousi and R. C. Myers. World-volume interactions on D-branes. Nucl. Phys. B542:73-88 (1999). hep-th/9809100. 12

[79] O. Aharony, S. S. Gubser, J. M. Maldacena, H. Ooguri and Y. Oz. Large $N$ Field Theories, String Theory and Gravity. Phys. Rept. 323:183-386 (2000). hep-th/9905111. 16

[80] E. Witten. Anti-de Sitter space, thermal phase transition, and confinement in gauge theories. Adv. Theor. Math. Phys. 2:505-532 (1998). hep-th/9803131. 19

[81] B. A. Burrington, J. T. Liu, L. A. Pando Zayas and D. VaMAN. Holographic duals of flavored $\mathcal{N}=1$ super Yang-Mills: Beyond the probe approximation. JHEP 02:022 (2005). hepth/0406207. 21, 158

[82] J. Babington, J. Erdmenger, N. J. Evans, Z. Guralnik and I. KIRsch. Chiral Symmetry Breaking and Pions in Nonsupersymmetric Gauge / Gravity Duals. Phys. Rev. D69:066007 (2004). hep-th/0306018. 24, 32, 33, 85, 86, 158

[83] A. Kehagias and K. Sfetsos. On Running Couplings in Gauge Theories from Type-IIB Supergravity. Phys. Lett. B454:270-276 (1999). hep-th/9902125. 32

[84] M. Gell-Mann, R. J. Oakes and B. Renner. Behavior of current divergences under $S U(3)$ x $S U(3)$. Phys. Rev. 175:2195-2199 (1968). 48 
[85] K. Skenderis and M. TAYlor. Branes in AdS and pp-wave spacetimes. JHEP 06:025 (2002). hep-th/0204054. 58

[86] M. R. Douglas. Branes within branes (1995). hep-th/9512077. 59

[87] N. Dorey, T. J. Hollowood, V. V. Khoze, M. P. Mattis and S. VANDOREn. Multi-instanton calculus and the AdS/CFT correspondence in $\mathcal{N}=4$ superconformal field theory. Nucl. Phys. B552:88-168 (1999). hep-th/9901128. 60

[88] E. Witten. Small Instantons in String Theory. Nucl. Phys. B460:541-559 (1996). hep-th/9511030. 60, 63

[89] M. R. Douglas. Gauge Fields and D-branes. J. Geom. Phys. 28:255-262 (1998). hep-th/9604198. 60, 63

[90] A. A. Belavin, A. M. Polyakov, A. S. Shvarts and Y. S. TYupkin. Pseudoparticle solutions of the Yang-Mills equations. Phys. Lett. B59:85-87 (1975). 61

[91] D. Oprisa and S. Stieberger. Six gluon open superstring disk amplitude, multiple hypergeometric series and Euler-Zagier sums (2005). hep-th/0509042. 64

[92] Z. Guralnik, S. Kovacs and B. Kulik. Holography and the Higgs branch of $\mathcal{N}=2$ SYM theories (2004). hep-th/0405127. 64

[93] Z. Guralnik, S. Kovacs and B. Kulik. AdS/CFT duality and the Higgs branch of $\mathcal{N}=2$ SYM (2005). hep-th/0501154. 64

[94] J. Babington, J. Erdmenger, N. J. Evans, Z. Guralnik and I. KIRsch. A Gravity Dual of Chiral Symmetry Breaking. Fortsch. Phys. 52:578-582 (2004). hep-th/0312263. 85

[95] L. Da Rold and A. Pomarol. Chiral symmetry breaking from five dimensional spaces. Nucl. Phys. B721:79-97 (2005). hepph/0501218. 88, 158 
[96] J. Erlich, E. Katz, D. T. Son and M. A. Stephanov. QCD and a holographic model of hadrons. Phys. Rev. Lett. 95:261602 (2005). hep-ph/0501128. 88, 158

[97] N. Evans and T. Waterson. Improving the infra-red of holographic descriptions of QCD (2006). hep-ph/0603249. 89, 158

[98] S. J. Gates, M. T. Grisaru, M. Rocek and W. Siegel. Superspace, or one thousand and one lessons in supersymmetry. Front. Phys. 58:1-548 (1983). hep-th/0108200. 95, 101, 102, 105, 107, 150

[99] I. L. Buchbinder and S. M. Kuzenko. Ideas and methods of supersymmetry and supergravity: Or a walk through superspace (1998). Bristol, UK: IOP (1998) 656 p. 101, 105, 108

[100] J. BAgGer and J. Wess. Supersymmetry and supergravity (1990). JHU-TIPAC-9009. 105

[101] J. Wess and J. BAGger. Supersymmetry and supergravity (1992). Princeton, USA: Univ. Pr. (1992) 259 p. 105

[102] J. Babington and J. Erdmenger. Space-time Dependent Couplings in $\mathcal{N}=1$ SUSY Gauge Theories: Anomalies and Central Functions. JHEP 06:004 (2005). hep-th/0502214. 136

[103] T. M. Apostol. Modular Functions and Dirichlet Series in Number Theory. Number 41 in Graduate Texts in Mathematics. SpringerVerlag, New York, 2 edition (1997). 144

[104] S. HöHne. Local Couplings in Quantum Field Theories. Master's thesis, Humboldt-Universität zu Berlin (2005). Diploma Thesis. 147

[105] T. Kugo and S. Uehara. Conformal and Poincaré Tensor Calculi in $\mathcal{N}=1$ Supergravity. Nucl. Phys. B226:49 (1983). 150

[106] M. Kruczenski, D. Mateos, R. C. Myers and D. J. Winters. Towards a holographic dual of large- $N_{c}$ QCD. JHEP 05:041 (2004). hep-th/0311270. 158 
[107] J. L. F. Barbon, C. Hoyos, D. Mateos and R. C. Myers. The holographic life of the eta'. JHEP 10:029 (2004). hep-th/0404260. 158

[108] N. J. Evans and J. P. Shock. Chiral dynamics from AdS space. Phys. Rev. D70:046002 (2004). hep-th/0403279. 158

[109] K. Ghoroku and M. Yahiro. Chiral symmetry breaking driven by dilaton. Phys. Lett. B604:235-241 (2004). hep-th/0408040. 158

[110] D. BAK and H.-U. YEE. Separation of spontaneous chiral symmetry breaking and confinement via AdS/CFT correspondence. Phys. Rev. D71:046003 (2005). hep-th/0412170. 158

[111] N. Evans, J. Shock and T. Waterson. D7 brane embeddings and chiral symmetry breaking. JHEP 03:005 (2005). hepth/0502091. 158

[112] T. Sakai and S. Sugimoto. Low energy hadron physics in holographic QCD. Prog. Theor. Phys. 113:843-882 (2005). hepth/0412141. 158

[113] R. Apreda, J. Erdmenger and N. Evans. Scalar Effective Potential for D7 Brane Probes which Break Chiral Symmetry (2005). hep-th/0509219. 158

[114] R. Apreda, J. Erdmenger, N. Evans and Z. Guralnik. Strong Coupling Effective Higgs Potential and a First Order Thermal Phase Transition from AdS/CFT Duality. Phys. Rev. D71:126002 (2005). hep-th/0504151. 158

[115] I. Brevik, K. Ghoroku and A. Nakamura. Meson mass and confinement force driven by dilaton. Int. J. Mod. Phys. D15:57-68 (2006). hep-th/0505057. 158

[116] T. Sakai and S. Sugimoto. More on a holographic dual of QCD. Prog. Theor. Phys. 114:1083-1118 (2006). hep-th/0507073. 158

[117] E. Antonyan, J. A. Harvey, S. Jensen and D. Kutasov. NJL and QCD from String Theory (2006). hep-th/0604017. 158 
[118] K. Ghoroku, T. Sakaguchi, N. Uekusa and M. Yahiro. Flavor quark at high temperature from a holographic model. Phys. Rev. D71:106002 (2005). hep-th/0502088. 158

[119] D. Mateos, R. C. Myers and R. M. Thomson. Holographic phase transitions with fundamental matter (2006). hep-th/0605046. 158

[120] T. Albash, V. Filev, C. V. Johnson and A. Kundu. A Topology-Changing Phase Transition and the Dynamics of Flavour (2006). hep-th/0605088. 158

[121] T. Albash, V. Filev, C. V. Johnson and A. Kundu. Global Currents, Phase Transitions, and Chiral Symmetry Breaking in Large $N_{c}$ Gauge Theory (2006). hep-th/0605175. 158

[122] A. Parnachev and D. A. Sahakyan. Chiral phase transition from string theory (2006). hep-th/0604173. 158

[123] O. Aharony, J. Sonnenschein and S. Yankielowicz. A holographic model of deconfinement and chiral symmetry restoration (2006). hep-th/0604161. 158

[124] A. Karch and A. O'Bannon. Chiral transition of $\mathcal{N}=4$ super Yang-Mills with flavor on a 3-sphere (2006). hep-th/0605120. 158

[125] S. Hong, S. Yoon and M. J. Strassler. Quarkonium from the fifth dimension. JHEP 04:046 (2004). hep-th/0312071. 158

[126] G. F. De Teramond and S. J. Brodsky. The hadronic spectrum of a holographic dual of QCD. Phys. Rev. Lett. 94:201601 (2005). hep-th/0501022. 158

[127] S. J. Brodsky and G. F. DE Teramond. Hadronic spectra and light-front wavefunctions in holographic QCD. Phys. Rev. Lett. 96:201601 (2006). hep-ph/0602252. 158

[128] H. Boschi-Filho and N. R. F. Braga. QCD / string holographic mapping and glueball mass spectrum. Eur. Phys. J. C32:529-533 (2004). hep-th/0209080. 158 
[129] H. Boschi-Filho and N. R. F. Braga. Gauge / string duality and scalar glueball mass ratios. JHEP 05:009 (2003). hepth/0212207. 158

[130] L. DA Rold and A. Pomarol. The scalar and pseudoscalar sector in a five-dimensional approach to chiral symmetry breaking. JHEP 01:157 (2006). hep-ph/0510268. 158

[131] J. P. Shock. Canonical coordinates and meson spectra for scalar deformed $N=4$ SYM from the AdS/CFT correspondence (2006). hep-th/0601025. 158

[132] J. P. SHock and F. Wu. Three flavour QCD from the holographic principle (2006). hep-ph/0603142. 158

[133] K. Ghoroku and M. Yahiro. Holographic model for mesons at finite temperature (2005). hep-ph/0512289. 158

[134] K. Ghoroku, N. Maru, M. Tachibana and M. Yahiro. Holographic model for hadrons in deformed $\mathrm{AdS}_{5}$ background. Phys. Lett. B633:602-606 (2006). hep-ph/0510334. 158

[135] K. Ghoroku, A. Nakamura and M. Yahiro. Holographic model at finite temperature with $R$-charge density (2006). hep-ph/0605026. 158

[136] A. Karch, E. Katz, D. T. Son and M. A. Stephanov. Linear confinement and AdS/QCD (2006). hep-ph/0602229. 158

[137] O. Andreev. 1/q2 Corrections and Gauge / String Duality (2006). hep-th/0603170. 158

[138] O. Andreev and V. I. Zakharov. Heavy-quark Potentials and AdS/QCD (2006). hep-ph/0604204. 158

[139] T. Hambye, B. Hassanain, J. March-Russell and M. SChVEllinger. On the $\Delta_{I}=\frac{1}{2}$ rule in holographic $Q C D$ (2005). hep-ph/0512089. 158

[140] J. A. M. Vermaseren. New features of FORM (2000). mathph/0010025. 160 
[141] K. Peeters. A Field Theory Motivated Approach to Symbolic Computer Algebra Preprint AEI-2006-037. 160

[142] K. Peeters. Cadabra: Reference Guide and Tutorial Preprint AEI-2006-038. 160 


\section{Personal Details}

$\begin{array}{llll}\text { Name } & \text { Johannes Große } & \text { Nationality } & \text { German } \\ \text { Date of Birth } & \text { March 31, 1978 } & \text { Parents } & \text { Annelie Große } \\ \text { Place of Birth } & \text { Berlin } & & \text { Eckhardt Blankertz } \\ \text { Marital Status } & \text { Married, 1 child } & & \end{array}$

\section{Education}

\begin{tabular}{|c|c|c|}
\hline Ph.D. & 04.2005 & $\begin{array}{l}\text { Continued at } \\
\text { Ludwig-Maximilians-Universität München }\end{array}$ \\
\hline Ph.D. & 04.2003 & $\begin{array}{l}\text { Started at } \\
\text { Humboldt-Universität zu Berlin }\end{array}$ \\
\hline Diplom & 12.2002 & $\begin{array}{l}\text { Thesis on "AdS/CFT Correspondence and } \\
\text { Renormalization Group Flows", } 117 \text { pages, } \\
\text { GPA 1.0, Supervisor Prof. Dieter Lüst, } \\
\text { Humboldt-Universität zu Berlin }\end{array}$ \\
\hline Vordiplom & 09.1999 & GPA 1.5, Humboldt-Universität zu Berlin \\
\hline A-Level & 1997 & GPA 1.0, Lise-Meitner-Oberschule Berlin \\
\hline Grammar School & 1990 & Hermann-Hesse-Oberschule \\
\hline Primary School & 1984 & Adolf-Glaßbrenner-Grundschule \\
\hline
\end{tabular}

